\title{
Phenomenological considerations of metacontrast masking
}

\author{
Dissertation \\ for the award of the degree \\ „Doctor rerum naturalium“ (Dr. rer. nat.) \\ of the Georg-August-Universität Göttingen
}

within the doctoral program Biology

of the Georg-August University School of Science (GAUSS)

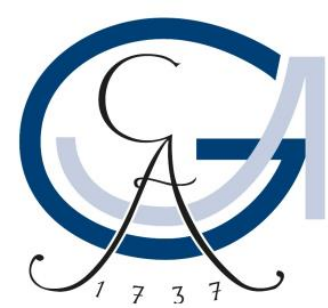

submitted by

Nora Meike Koster

from Kirchheimbolanden,

Goettingen, 2019 


\title{
Phenomenological considerations of metacontrast masking
}

\author{
Dissertation
}

zur Erlangung des mathematisch-naturwissenschaftlichen Doktorgrades

"Doctor rerum naturalium" (Dr. rer. nat.)

der Georg-August-Universität Göttingen

im Promotionsprogramm Biologie

der Georg-August Universität School of Science (GAUSS)

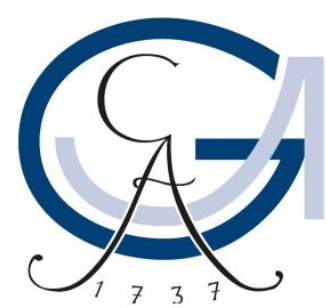

vorgelegt von

Nora Meike Koster

aus Kirchheimbolanden

Göttingen, 2019 


\section{Betreuungsausschuss}

Prof. Dr. Uwe Mattler

Abteilung für Experimentelle Psychologie, Georg-Elias-Müller-Institut für Psychologie, Georg-August-Universität Göttingen

Prof. Dr. Hannes Rakoczy

Abteilung für Biologische Entwicklungspsychologie, Georg-Elias-Müller-Institut für Psychologie, Georg-August-Universität Göttingen

Dr. Thorsten Albrecht

Abteilung für Experimentelle Psychologie, Georg-Elias-Müller-Institut für Psychologie, Georg-August-Universität Göttingen

Mitglieder der Prüfungskommission

Referent: Prof. Dr. Uwe Mattler

Koreferent: Prof. Dr. Hannes Rakoczy

$\underline{\text { Weitere Mitglieder der Prüfungskommission }}$

apl. Prof. Dr. York Hagmayer

Abteilung für Kognitionswissenschaft und Entscheidungspsychologie, Georg-Elias-MüllerInstitut für Psychologie, Georg-August-Universität Göttingen

Prof. Dr. Annekathrin Schacht Abteilung für Affektive Neurowissenschaft und Psychophysiologie, Georg-Elias-MüllerInstitut für Psychologie, Georg-August-Universität Göttingen

Prof. Dr. Stefan Treue Abteilung für Kognitive Neurowissenschaften, Georg-August-Universität Göttingen, Deutsches Primatenzentrum

Prof. Dr. Melanie Wilke Abteilung für Kognitive Neurologie der Universitätsmedizin Göttingen

Tag der mündlichen Prüfung: 13.03.2019 


\section{Acknowledgements}

My sincere thanks go to all my colleagues for the very nice cooperation over the past years. Passionate discussions and a good portion of humor have made working very pleasant. I am very grateful for the fruitful cooperation that and I will miss you.

Especially my supervisors, Uwe Mattler, Hannes Rakoczy and Thorsten Albrecht helped me a lot to advance my work with constructive criticism, emotional support and inspiration. I have always found an open ear and the willingness to support me with problems. Thank you very much for your support over the last three years.

Of course, I would never have been able to work without the support of the student assistants who accompanied me during my doctorate: Alexander Kraut, Judith Diele, Charlotte Fink, Selin Altiok und Katja Lorenz. Thank you very much for your great cooperation.

My thanks also go to my family and friends, who cheered me up in difficult times and without whom life would be half as beautiful. In particular I would like to thank my husband, who is always there for me with help and advice, who believes in me and supports me. I would like to dedicate the last thought to my daughter who made it possible to finish my work in spite of her presence. I am very happy that you are with us. 


\section{Summary}

This thesis enriches the understanding of metacontrast masking as multidimensional phenomenon on the basis of phenomenological, behavioral as well as neurophysiological data. The phenomenological investigations (Experiment 1-2) depict the perception of seven qualitative different target aspects, regarding the perceived temporal distance of both stimuli (Target inside Mask, Target before Mask), the perceived contrast (Dark Target, Bright Target, No Target) and apparent motion perceptions (Rotation, Expansion). The unique relationships of these perceptions with SOA and Congruency showed that the experience of the target varies qualitatively in a metacontrast masking paradigm. This contradicts with the assumption that variations of the SOA influence the awareness of the target only quantitative, as indicated by one-dimensional objective and subjective measures. The underestimation of the target perception by objective measures is also indicated by the relationship between discrimination performance and the perception of the different target aspects (Experiment 2). In accordance to the phenomenological variety in the perception of the target, evidence for different processes was provided by the results of Experiment 3. Regarding these results, it seems to be inappropriate to conceptualize metacontrast masking as a unitary process underlying the whole range of SOAs. Instead, metacontrast can be defined as multidimensional phenomenon with three different processes underlying short and long SOAs. The process underling short SOAs was associated with two perceptions, which were found to depend on each other, the perceptions of a target integrated inside the mask and an expansion. At long SOAs, evidence for two independent processes was found, eliciting the perceptions of a rotation and a target temporally segregated from the mask. Finally, electrophysiological results supported the assumption that the perceptions at long SOAs were reflected by two distinct mechanisms, since unique neural signatures were found for both (Experiment 4). 


\section{Contents}

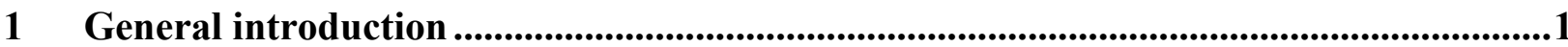

1.1 Subjective experiences in consciousness research .......................................... 1

1.2 Phenomenology and the compatibility with scientific approaches ........................... 3

1.2.1 Differentiation between Phenomenology and Introspection ............................... 4

1.2.2 Differentiation between traditional Phenomenology and Phenomenology as

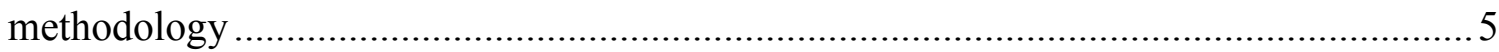

1.2.3 A historical development of subjective measures in psychology ......................... 9

1.3 Subjective experiences in metacontrast masking paradigms ................................. 13

1.3.1 Theories about the mechanism of metacontrast masking .................................. 16

1.3.2 Theories of lateral inhibition and feedforward processing ................................ 16

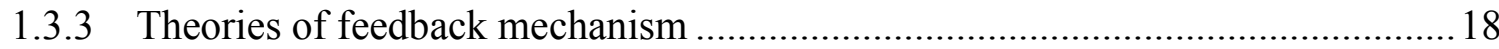

1.3.4 Summary

1.4 Motivation to consider phenomenology in a metacontrast masking paradigm ........24

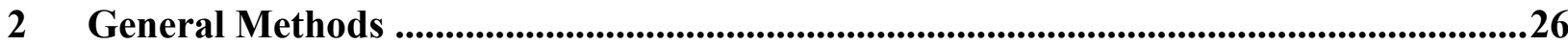

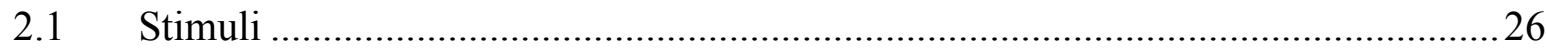

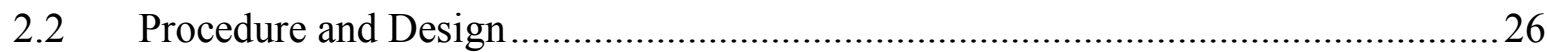

3 Visual perception is rich. Phenomenological evidence from metacontrast masking ....28

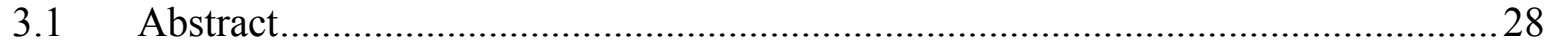

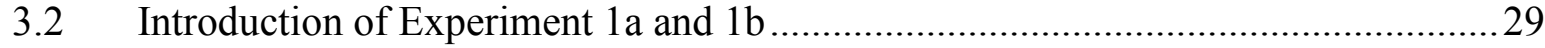

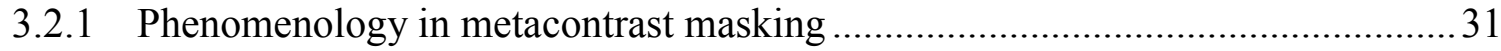

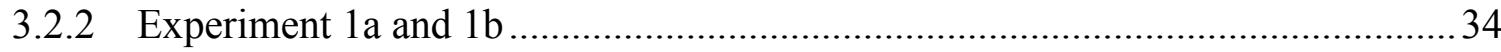

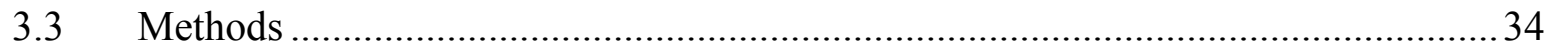

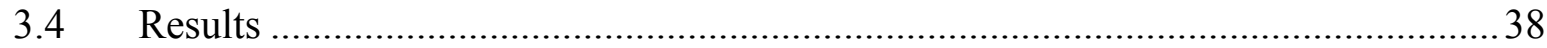

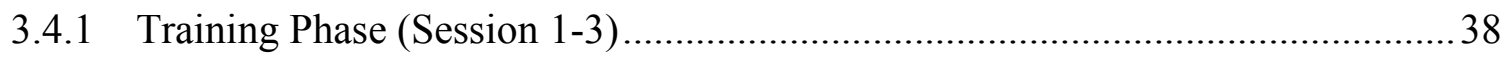

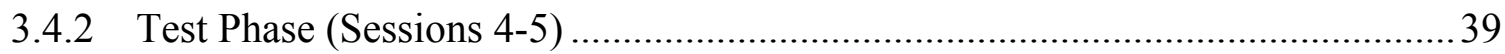


3.5 Discussion.

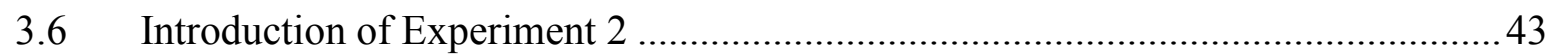

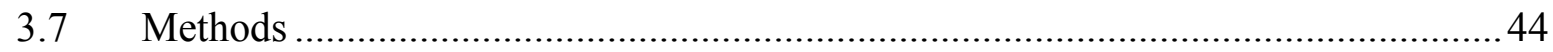

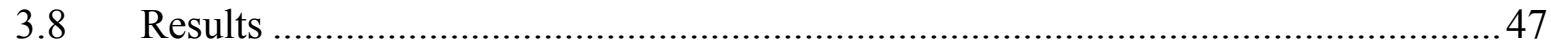

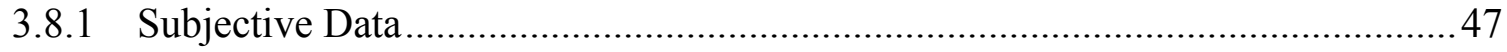

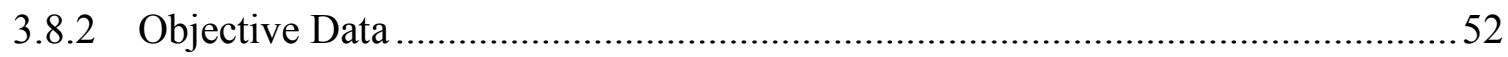

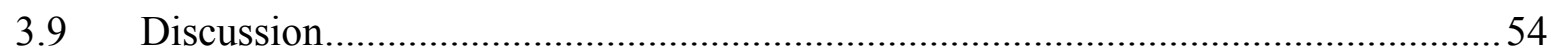

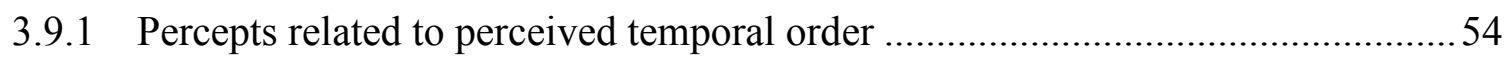

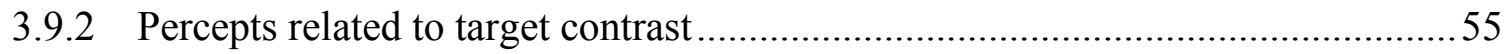

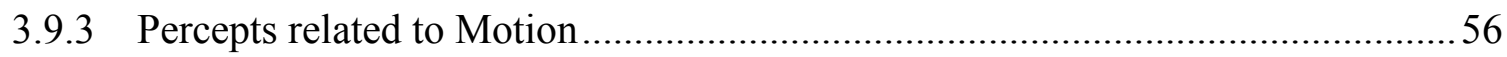

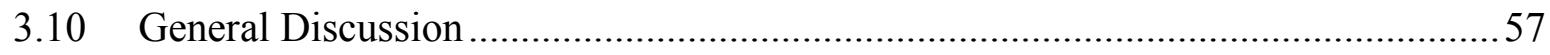

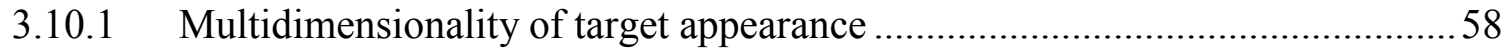

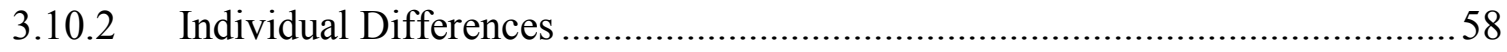

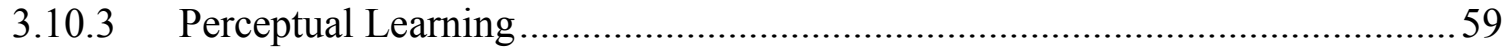

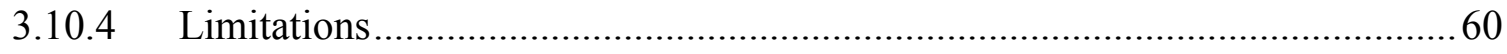

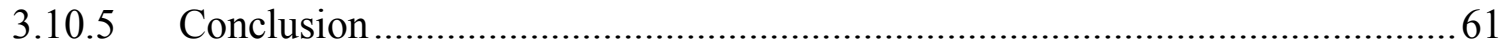

\section{Phenomenological evidence for multiple processes underlying metacontrast} masking................................................................................................................................................................63

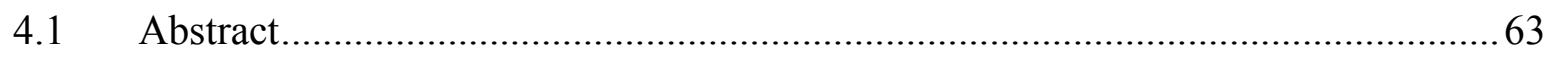

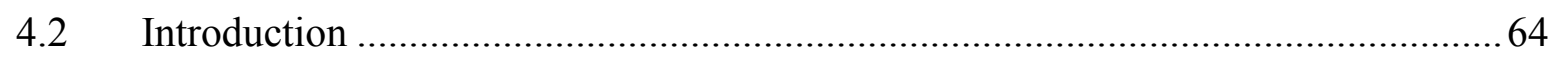

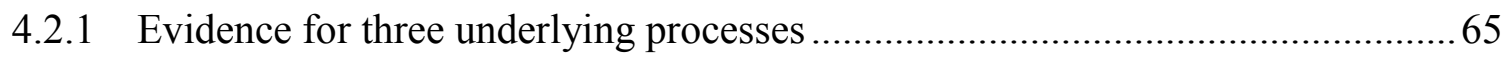

4.2.2 Phenomenological investigations to examine underlying processes ...................66

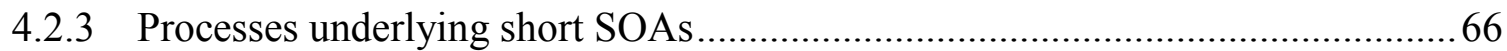

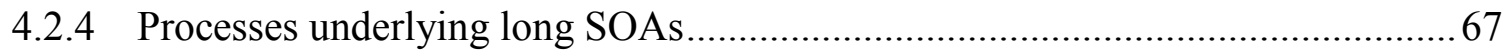

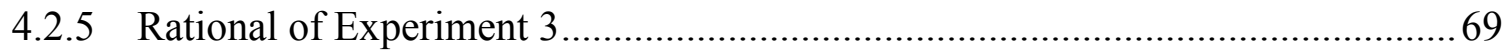

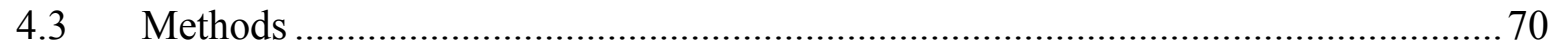

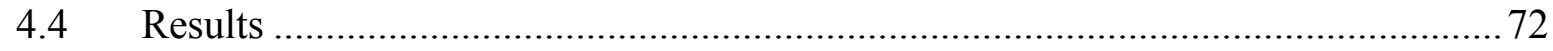


4.4.1 Dependency of Expansion from Target inside Mask ........................................ 72

4.4.2 Dependency of Target inside Mask from Expansion ........................................ 75

4.4.3 Independency of Rotation from Target before Mask ....................................... 78

4.4.4 Independency of Target before Mask from Rotation ...................................... 84

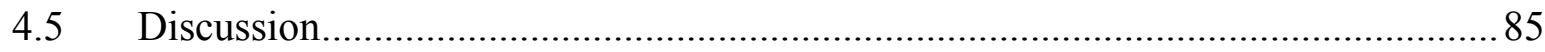

4.5.1 Evidence for one processes underlying short SOAs...................................... 85

4.5.2 Evidence for two processes underlying long SOAs ...................................... 88

4.5.3 Assumptions about the type of processes underlying metacontrast masking at

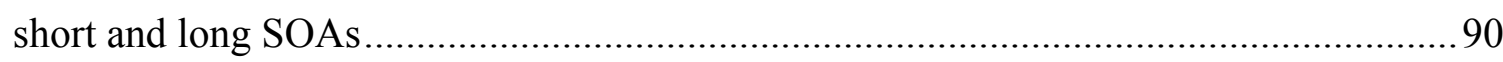

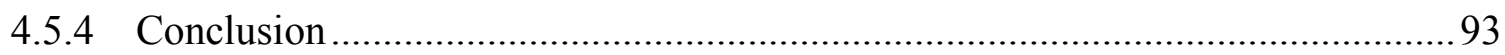

5 Neurophysiological evidence for the multidimensionality of metacontrast masking....95

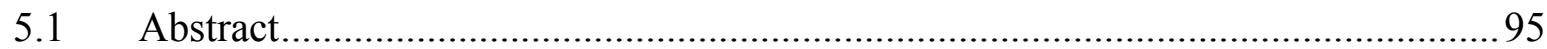

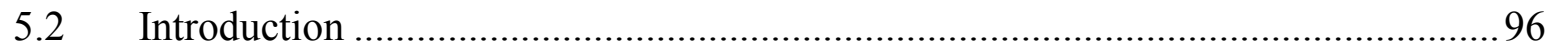

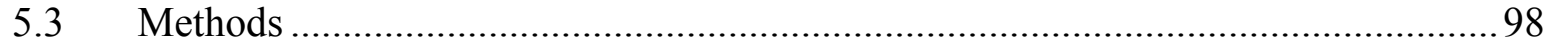

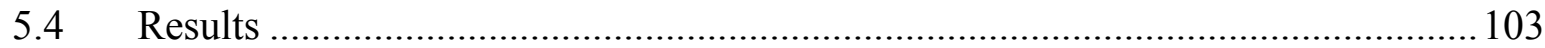

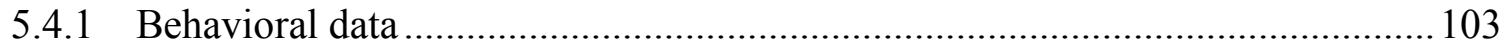

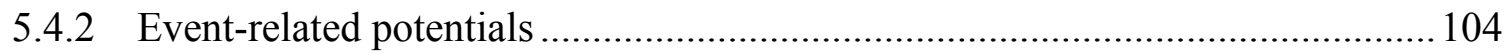

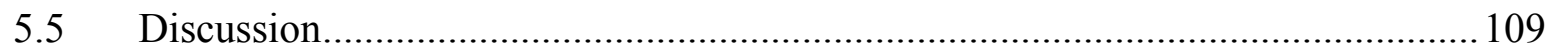

5.5.1 Evidence for two distinct neural signatures................................................ 109

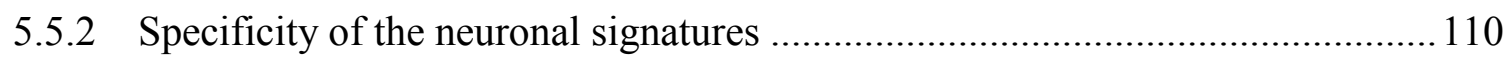

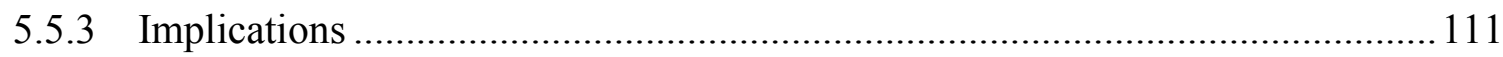

5.5.4 A cautious interpretation of the functional significance of both neural signatures 113

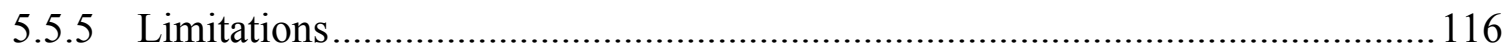

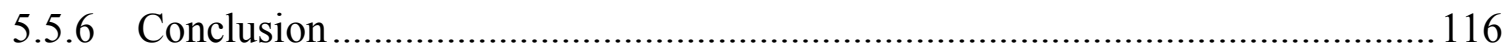

6 Overall Discussion

6.1 A methodological evaluation of the phenomenological investigation ..................120 


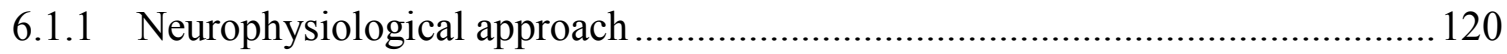

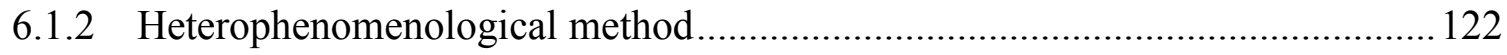

6.1.3 Methodological limitations and summary ................................................ 123

6.2 Phenomenological insights into metacontrast masking................................... 124

6.2.1 Subjective experience and their relation to objective performance.................... 127

6.2.2 Generalizability of the perceptions to other masking paradigms ...................... 131

6.3 Consideration of metacontrast mechanisms on the basis of phenomenological data 136

6.3.1 Processes underlying metacontrast masking .............................................. 138

6.3.2 Low-level masking mechanism at short SOAs............................................... 140

6.3.3 High-level masking mechanism at long SOAs ............................................... 142

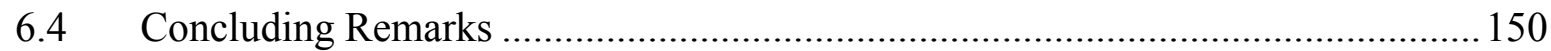

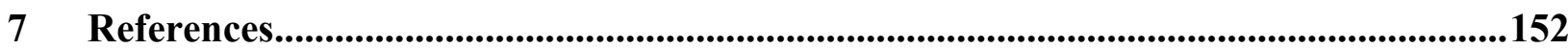

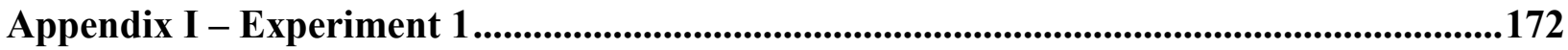

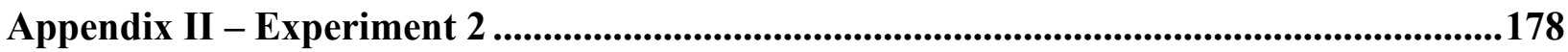

Appendix III - Experiment 3.............................................................................................183

Appendix IV - Experiment 4 ............................................................................................................185

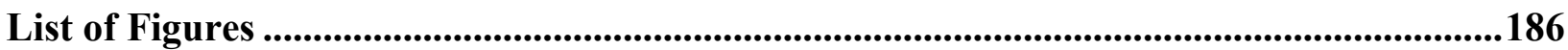

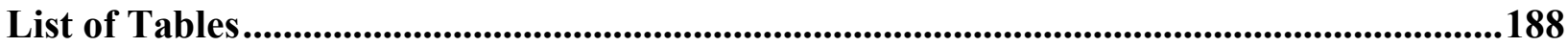

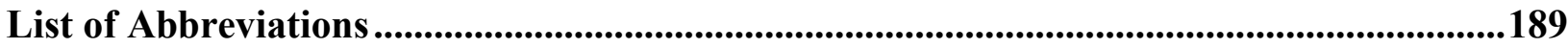



„Experience is the most central and manifest aspect of our mental lives, and indeed is perhaps the key explanandum in the science of the mind" (Chalmers, 1995, p. 206).

\section{General introduction}

\subsection{Subjective experiences in consciousness research}

The importance of subjective data in consciousness research has been highlighted by philosophers of mind for years. Chalmers stated that "the really hard problem of consciousness is the problem of experience" (1995, p. 201). His formulation of the so-called hard problem of consciousness was built on Nagel (1974), who coined the remark "what is it like to be a bat". On the one hand, this remark outlines that experiences have a private and subjective character. The bat is experiencing the world with the echo-locating system in a very subjective, bat-like way. On the other hand, Nagel's sentence transports the dilemma inherent to consciousness research that the subjective, first-person experience, can never be captured from a third-person perspective. This implies that it is impossible to understand the experience of the bat as an observer.

Chalmers (1995) picked up this dilemma by differentiating between the easy and the hard problem of consciousness. The easy problem deals with the question how to explain cognitive functions and abilities associated with consciousness, for example access, information integration or report. Even if several easy problems have not yet been solved, he assumed that standard methods of cognitive science are generally sufficient to explain them. For example, cognitive functions can be explained by defining underlying computational as well as neural mechanisms. In contrast, Chalmers (1995) pointed out that the hard problem is not a matter of functions, it goes beyond. The hard problem addresses how functions are associated with experience. In accordance with Nagel's (1974) assumptions, Chalmers pointed out that there is an explanatory gap between functions and experiences, causing the problem that the private, subjective aspects of experiencing cannot be explained with any existing methods of cognitive or neurobiological research. As a consequence, Chalmers (1995) criticized any physical theory explaining consciousness only by structures and functions, as insufficient to tackle the hard problem. Instead, he asked for a nonreductive theory of consciousness which defines experiences as the fundamental and connects features of the physical world with features of experiences. Since experiences cannot be observed with 
scientific investigations, he suggested relying on verbal reports which describes the experiences.

Despite Nagel's (1974) skepticism about the scientific addressability of the first-person perspective, he did not fundamentally reject materialistic approaches, but only those which did not take into account the subjective character of experiences. Instead, he highlighted the importance to explore the subjective character of experience, to know what a physical theory must be able to explain. He described this process of capturing the experiences as an objective understanding of the mental. This claim highlighted that Nagel considered subjective experience as the basic level of explanations any theory of consciousness should be built up on.

Searle's (2000) understanding of consciousness is very similar to Nagel's. Searle (2000) distinguished consciousness from other phenomena on the basis of three aspects, qualitativeness, subjectivity and unity. The first aspect, qualitativeness, implies that each state of consciousness has its own, inherent qualitative feature. So each experience is associated with a different qualitative sensation. The second aspect, subjectivity means that conscious states are always perceived by someone. Unity defines the combination of different conscious sensations experienced simultaneously. Despite the subjective first-person perspective of consciousness, Searle (2000) warned against understanding consciousness as a phenomenon which cannot be investigated with scientific or epistemological methods. Instead, Searle (2000) pointed out that third-person objective processes realized in the brain structures are causing the subjective experiences. He called his approach a biological naturalism and pointed out that one major task of consciousness research is to explain how brain structures are causing subjective experiences. This statement can be understood as an assumption of two strongly interrelated aspects, which stand in a clear causal relation. Both needed to be understood to explain consciousness, the subjective experiences as well as their neurological basis. In addition, Searle (2000) stated that consciousness cannot be explained without taking into account subjective aspects as an inherent feature of consciousness that differentiate it from other biological phenomena. Searle's definition of consciousness points out how indispensable it is to capture subjective experiences for the investigation of consciousness. Based on his view, without subjective experiences, anything would have been measured, but not consciousness, since the subjective, qualitative experience is the essence of consciousness.

These considerations clarify the importance of subjective experience for the formulation of an appropriate and comprehensive theory of consciousness. Accordingly, statements about functions can be insufficient without considering subjective aspects of 
experience. According to Chalmers definition of first-person data, as private, inner states only the subject itself has a privileged access to, verbal descriptions are never referring to the firstperson's experience. Therefore, each attempt to verbalize subjective, first-person experiences would only transport objective, third-person data. Verbal descriptions as well as other behavioral responses, referring to first-person experience, are defined as subjective data. Consequently, the measurement of subjective data is the best approximation to the experience of the subject. Subjective data is distinguished from objective data, because subjective information cannot be evaluated as true or false, whereas objective data is any kind of performance measure with a clear wright or wrong evaluation (Overgaard, Jensen, \& Sandberg, 2009). Furthermore, this work only focuses on problems Chalmers (1995) defined as easy and the reply to the hard problem is left aside.

\subsection{Phenomenology and the compatibility with scientific approaches}

Beside philosophical considerations, about the necessity to capture subjective experiences, there is also a methodological controversy, if and how to consider the subjectivity of experiences in psychology, for example in cognitive science. There are two common approaches: Introspection and Phenomenology.

Phenomenology is a widely used term with several meanings. First, the phrase "the phenomenology in an experimental setting" simply refers to the qualitative experiences of the subject. Gallagher and Zahavi (2008) called this definition of phenomenology non-technical since it is merely another term for experience. Dennett provided a broad definition of phenomenology as all objects that inhabit conscious experience (Dennett, 1991, p. 44). In the present investigation, the term phenomenology, with a small letter ("p"), refers to the quality of experiences.

Second, in the nineteenth century, Phenomenology designates a philosophical tradition, invented by Husserl (Gallagher \& Zahavi, 2008) that provided a descriptive study of subjective matters (Dennett, 1991, p. 44).

Third, to integrate a phenomenological methodology in cognitive science several different attempts to naturalize Phenomenology have been developed. In other words, naturalized Phenomenology is the attempted to use third-person objective approaches to measure subjective experiences. In the present investigation the term Phenomenology does not describe the philosophical attitude unless explicitly stated. Instead, it encompasses the methodological implementation of the perception of subjective experience in a standardized, 
experimental setting. The term Phenomenology used in a philosophical or methodological sense, it is written with a capital letter ("P").

In the following Sections the philosophical as well as the methodological understandings of Phenomenology are contrasted in regard of their compatibility with the assumptions of a scientific approach. In particular it will be outlined if traditional Phenomenology, as well as so-called naturalized Phenomenology can be incorporated in a scientific framework. But as a first step, Phenomenology is distinguished from Introspection. Both terms are often strongly interrelated and a comparison allows grasping both concepts more clearly.

\subsubsection{Differentiation between Phenomenology and Introspection}

From a philosophical as well as methodological point of view Phenomenology is not just a special form of Introspection (Gallagher \& Zahavi, 2008; Varela, 1996), even if some authors use both terms synonymously (Gallagher, 2003; Timmermans \& Cleeremans, 2015). In a weak sense, Introspection can be understood as any kind of report the participant is giving about his experience (Gallagher \& Sørensen, 2006), because each answer to a stimulus requires an insight into the own perception. Based on this wide definition nearly any kind of answer in an experimental setting, from behavioral responses to verbal reports, can be interpreted as introspective. Since this weak sense of introspection is too unspecific to capture methodological implementations, a more specific definition is required.

Wilhelm Wundt (1832-1920) who founded the first experimental psychological institute in Leipzig (1879) used introspection as a methodology to study consciousness (Velmans, 2007). A description of a prototypical experimental setting in the sense of Wundt is given in Section 1.2.3. For introspective methods the focus of attention is directed at the own mental states and the subjective experience, rather than at the stimulus itself (Gallagher \& Sørensen, 2006). This description of introspection coincides with the definition of William James (1842-1910), who defined Introspection as "looking into our own minds and reporting what we there discover" (James, 1890a, p. 185). In addition, he stated pure sensations as abstractions that encompass two things: First, simple qualities and properties of an object and second knowledge about the relation of the object with other things in the world (James, 1890 b, p. 3). Consequently, an introspective approach tries to capture not just the experience of the stimulus, but also the subject observing the own inner states while experiencing the stimulus. 
Phenomenology can be distinguished from introspective approaches. Whereas introspection can be interpreted as a process of understanding and verbalizing the own mental states, Phenomenology is not interested in the beliefs, opinions or subjective interpretations the observer has about his experience, but only in the experience itself (Gallagher \& Sørensen, 2006). A phenomenological methodology is not capturing private, subjective thoughts but "intersubjectively accessible modes of appearance" (Zahavi, 2003, p. 54). Phenomenology investigates intersubjective commonalities in experience and attempts to identify invariant structures of experience associated with certain conditions of an experiment (Gallagher \& Sørensen, 2006). Therefore, a narrow interpretation of Phenomenology would reject it as a pure methodology of capturing subjective experience. Instead, Phenomenology can be defined an attempt to grasp the nature of objectivity (Gallagher \& Zahavi, 2008).

In this thesis both terms are not used in the philosophical meaning, but rather as descriptions of methodologies to measure subjective experiences in an experimental setting. If reference is made to a particular methodology, the above mentioned differences are taken into account and accordingly both terms are distinguished from each other. But if only the collection of subjective experience is described both terms are used interchangeable.

\subsubsection{Differentiation between traditional Phenomenology and Phenomenology as methodology}

It is questionable whether classical Phenomenology can be integrated into a scientific framework or if this approach is impossible due to irreconcilable assumptions. First, the incompatibility is demonstrated by the fact that a traditional phenomenologist would deny the differentiation between the reality of an object located in the outside and the subjective appearance of the object to the observer (Gallagher \& Zahavi, 2008). In contrast, already the description of psychology by James included the distinction between an object in the real world with its features and a mental perception of this object, which could misrepresent other features (1890a, pp. 183-185). He stated that for a psychologist both are objects of an observation. The differentiation of James between the real object and its mental representation is still a central assumption of psychological research. Second, Overgaard (2004) concluded that traditional Phenomenology is not combinable with any scientific approach which accepts mind-brain correlations, since Phenomenology is taken verbal reports of experiences as the fundamental ontological level of consciousness. Consequently, classical Phenomenology in terms of Husserl will never accept any reductionistic explanation of consciousness. In 
contrast, cognitive neuroscience is based on the assumption that functions are caused by brain activity. Even in philosophy of mind it is not unusual to represent this kind of naturalism. For example Searle's (2000) notion of a causal relation between neural activity and experiences includes a reduction of experience to a neural level. Therefore, this contradicts with the nonreductionistic assumption of traditional Phenomenology, because if brain structures are used to explain mental states, consciousness would explain nothing by itself (Overgaard, 2004).

To overcome the incompatibility between the traditional understanding of Phenomenology and natural science, Overgaard (2004) advocated an approach which he called naturalizing Phenomenology. He described the idea of naturalized Phenomenology not as a philosophical position, but as a method to systematically describe the experiences of the subject, embedded in a scientific framework. There are several different methodologies to naturalize Phenomenology, but for the sake of brevity only a few are highlighted. These approaches seem to be promising to incorporate naturalized Phenomenology as a methodology in a scientific framework of cognitive psychology or cognitive neuroscience.

Varela (1996) defined a phenomenological methodology for capturing conscious, which he called Neurophenomenology. Varela's approach faces similar incompatibilities with psychological methodologies as traditional Phenomenology. First, because of the assumption about the irreducible nature of consciousness (Varela, 1996). Second, because Varela and Shear (1999) claimed that the hard problem can be tackled by this approach. Even though these claims may be questioned, Neurophenomenology can be considered as promising methodology to capture subjective experiences in an experimental setting. The working hypothesis of Neurophenomenology, stated that "phenomenological accounts of the structure of experience and their counterparts in cognitive science relate to each other through reciprocal constraints" (Varela, 1996, p. 343). The particularity of this claim is the reciprocity between subjective experiences and third-person data, which were considered as equally important. This highlights the importance of subjective data for the investigation of cognitive processes. The working hypothesis also includes the belief that subjective experiences are an important source for empirical questions and may provide an understanding of third-person data, for example neurophysiological data.

Varela (1996) defined four important aspects of phenomenological approaches, which he called phenomenological reduction. First, he defined an appropriate attitude for phenomenological experiments. This means one should avoid turning attention to automatic thoughts or beliefs about the experiences while observing, but stay by the experience itself. Second and most important, subjects should attain intimacy with their own experiences. To 
achieve this, they should become familiarized with variations in the appearance of an object under different experimental conditions. Third, subjects should be trained to communicate observed similarities in experience, through language or sketches. Varela (1996) defined these observed similarities as invariants. Fourth, to fulfill the other aspects, it is necessary to maintain stability in the perception, which is achieved by training. Lutz, Lachaux, Martinerie, and Varela (2002) provided an exemplary implementation of the neurophenomenological reduction in a neurophysiological experiment. Lutz et al. (2002) presented a threedimensional visual illusion (autostereogramm) and asked subjects to press a button as soon as they perceived the figure completely and give a phenomenological description about their own experience on each trial. After a training, where subjects repeatedly saw the stimuli to gain stability in the perception and to be able to describe their invariants, EEG sessions followed. On each trial they labeled their experienced based on their invariants, which were used to divide the trials into three phenomenological clusters, shared by participants. These clusters categorized the subjective degree of preparation, for example "ready", "present" and "well-prepared" as one cluster or "unprepared" as another cluster. Behavioral as well as neurophysiological results show that reaction times as well as prestimulus oscillations were modulated in dependence of the phenomenological clusters. On trials where participants reported to feel well-prepared, a higher local and global neural synchrony was found compared to unprepared trials. Lutz et al. (2002) interpreted their approach as an extension of traditional cognitive psychology, by measuring subjective experience of trained subjects in a trial-by-trial manner, which allows to characterize neural correlates of mental states.

Another but very different attempt to naturalize Phenomenology has been formulated as Heterophenomenology by Dennett (2003). He stated that Heterophenomenology is already incorporated in any kind of research studying consciousness in a scientific way. Dennett (2007) described Heterophenomenology as a third-person approach which brings together subjective aspects of consciousness with scientific approaches. Instead of considering Heterophenomenology as a theory, he described it as a method which organizes the subjective reports to clarify, what must be explained (Dennett, 2003). Dennett (2003) pointed out that the heterophenomenological world, which entails the beliefs of a subject about his or her consciousness experience, should not be confounded with the real world. Thus, he considered statements about experiences as something else than the experiences themselves. According to him, statements should be interpreted as abstractions, which give evidence for beliefs about experience. The utterances of a person was considered as raw, uninterpreted and primary data, a phenomenologist should observe (Dennett, 2007). For this kind of heterophenomenological 
observation, a neutral attitude towards the descriptions was regarded as fundamental (Dennett, 1991). This means, the Heterophenomenologist should neither challenge nor accept the reports of the subjects, but rather interpret the descriptions in a meaningful way (Dennett, 1991).

Gallagher and Sørensen (2006) contrasted these two methodologies. They proposed that the consideration of the four aspects of phenomenological reduction formulated by Varela (1996) is necessary to measure the subjective aspects of experiences. They equated Neurophenomenology with phenomenological approaches and concluded that only this approach is able to fulfill phenomenological requirements. Heterophenomenology was associated with introspective approaches, since both attempts are not only focusing on the experiences as Phenomenology, but on thought and beliefs about the experiences, too. However, Gallagher and Sørensen (2006) criticism on Heterophenomenology can be doubted.

First, the categories defined by the subjects in the neurophenomenological method are biased by individual variability in perception, linguistic abilities or the focus of attention and so on. Therefore, the mere consideration of individual descriptions of perceptions might be insufficient to make generally valid statements.

Second, Gallagher and Sørensen (2006) criticized Heterophenomenology to wash out any subjective factor, since perceptions are mostly measured by predefined categories, which are not based on subjective experiences. In accordance, Varela and Shear (1999) criticized Heterophenomenology as an approach which tries to collect first-person data while being attached only to a third-person methodology. On the one hand, the criticism is justified because the use of predefined categories that have not been validated on the basis of subjective reports contradicts phenomenological principles. On the other hand, in order to make statements about how experience is related to variations in experimental design, commonalities in perception between subjects must be considered.

Instead of advocating one of the two approaches, the combination of both seems to be a promising approach to satisfy phenomenological methodologies and additionally fulfill the requirements of a scientific framework. In a first step, a neurophenomenological approach allows the collection of descriptions of subjective experiences depending on the condition in a perceptual task. Afterwards, perceptual categories can be defined based on communalities in the descriptions between participants. In a second step, a heterophenomenological approach enables to validate the perceptual categories by searching for similarities in experience associated with experimental conditions. This last step helps to objectify the data collection 
and allows statements about cognitive or neurophysiological processes related to changes of the experience.

\subsubsection{A historical development of subjective measures in psychology}

This section gives a brief historical overview of the measurement of subjective experiences in psychology and consciousness research. For the sake of clarity, in this section the collection of subjective experience is only termed as Introspection, without explicitly distinguishing between the different methods presented in the previous sections.

Already in the beginning of the nineteenth century Wundt defined the study of consciousness as the major task of psychology (Velmans, 2007). At this time, subjective measures were considered to be the source to examine consciousness, which has been undertaken by several authors, for example William James, Edmund Husserl, Wilhelm Wundt and Theodor Fechner (Gallagher \& Zahavi, 2008). The early experimental studies of psychology established introspection as a method to get an insight on the mental processes (Gallagher \& Zahavi, 2008). In the beginning of the twentieth century the methodology of introspection was improved for example by two students of Wundt, Titchener and Külpe. Standardized experimental investigation of introspection were established by offering specific tasks with identical condition for each subject as well as detailed instructions (Vermersch, 1999). Witt (2010) offered a description of a prototypical experimental setting in the sense of Wundt. The experiment was performed in 1907 by Edward Scripture, one of Wundt's doctorate students at the laboratory in Leipzig. Either as written or spoken words or objects, optical, acoustic and tactile stimuli were presented for mostly four seconds and needed to be palpated afterwards. The presentation of the next object was verbally signalized two seconds before the presentation. Participants were sitting in a dark room. Their task was to associate freely. Witt (2010) also gave an example of an association to the spoken word "palm": "It reminds me of a landscape in the tropics, stemming from a picture". The experiment had the aim to capture the process of associations, as well as the relation between stimulus and sensation. Thereby, Wundt's introspective experiments were mostly performed by systematically trained subjects and controlled stimuli, which should help to replicate the results (Overgaard, 2006).

But despite these developments, the importance of subjective measures declined. First, most of the hypotheses could not be confirmed (Vermersch, 1999). Second, there was a huge variability in results between different laboratories, which was due to differences in the exact 
methodology, mainly the amount and way of training (Velmans, 2007). These problems resulted in strong criticism and displacement of introspective approaches by behavioristic methods (Velmans, 2007). A common behavioristic point of view was to reject introspection as an adequate scientific method (Costall, 2006). But also a general change in the understanding of psychology as a natural science took place at this time. For example Watson (1931), a proponent of behaviorism, defined psychology as a purely objective experimental branch of natural science. He designated introspective methods as unreliable and requested to discard consciousness as a topic for psychological research (Watson, 1931). Behaviorist defined cognitive processes as ontological irrelevant, because they can be reduced to observable actions (Velmans, 2007). Therefore, for a behaviorist it is equally informative to state, "He is eating", compare to "He is eating, because he is hungry" (Velmans, 2007, p. 223). But this assumption leaves aside that not every mental state can be reduced to and explored by observable behavior.

The cognitive revolution brought a change and transformed the study of consciousness back into a scientific addressable topic (Gallagher \& Varela, 2003). In cooperation with computer science and brain research, new methods were developed to get an understanding of consciousness. But the skepticism towards introspective methods remained (Vermersch, 1999). They were described as unreliable and invalid (Nisbett \& Wilson, 1977). Therefore, in the 1970s and 1980s, objective performance and behavioral responses were favored over subjective measures to state about consciousness (Ramsøy \& Overgaard, 2004). Experimental results increased skepticism about the reliability of subjective experiences. In particular, change blindness paradigms showed that even large and salient changes between two scenes went unnoticed, despite the subjective impression of a detailed and naturalistic perception of the environment (for review see Simons, 2000).

However, subjective measures have never completely disappeared from psychological research (Costall, 2006). At the beginning of the twenty-first century, they were even rehabilitated as their importance for the study of consciousness was rediscovered. The importance of subjective measurements was substantiated by the findings of blindsight patients who suffered from a damage of the visual striate cortex, causing cortical blindness in the contralateral visual half field (Timmermans \& Cleeremans, 2015, p. 33). Weiskrantz, Barbur, and Sahraie (1995) showed that a blindsight patient was able to correctly discriminate a visual stimulus in a forced-choice task, even if he subjectively reported not to be aware of the stimulus. The phenomenon of blindsight was interpreted as evidence for subliminal processing, falsely detected by the objective task as conscious perception (Timmermans 
\& Cleeremans, 2015). These results stimulated a debate about the necessity to capture subjective data and not only performance, which could also been influenced by unconscious processing. Also experimental results with healthy subjects revealed a similar dissociation between subjective measures and objective performance (e.g. Jannati \& Di Lollo, 2012; Lau \& Passingham, 2006; Sandberg, Bibby, Timmermans, Cleeremans, \& Overgaard, 2011; Sandberg, Timmermans, Overgaard, \& Cleeremans, 2010). In these studies participants reported subjectively not to have seen the barely visible stimulus, however, the objective task indicated above-chance performance for these trials. The authors concluded that objective measures erroneously detect unconscious processing, which makes them unsuitable for investigating conscious perception. Based on previous evidence for objective measures outperforming subjective, Merikle proposed the working hypothesis that "subjective measures can provide an adequate indication of the presence or absence of relevant conscious experiences" (1992, p. 794), whereas objective measures may fail to exclusively demonstrate conscious perception. Consequently, in the beginning of the twenty-first century several subjective measures have been developed, which either capture the clearness of the perception of a stimulus, for example the perceptual awareness scale (PAS) (e.g. Ramsøy \& Overgaard, 2004; Sandberg et al., 2011; Sandberg et al., 2010; Sandberg, Bibby, \& Overgaard, 2013; Schwiedrzik, Singer, \& Melloni, 2011), as well as binary (e.g. Jannati \& Di Lollo, 2012; Lamy, Salti, \& Bar-Haim, 2008; Lau \& Passingham, 2006) or gradual judgments about the visibility of the stimuli (e.g. Del Cul, Baillet, \& Dehaene, 2007; Sergent \& Dehaene, 2004). Other kinds of subjective measures captured metacognitive judgment about the own performance, for example the confidence about a previous decision in the objective task (e.g. Szczepanowski \& Pessoa, 2007; Szczepanowski, Traczyk, Wierzchoń, \& Cleeremans, 2013; Zehetleitner \& Rausch, 2013) or the amount of money the participants are willing to bet on their decision in the objective task (e.g. Dienes \& Seth, 2010; Persaud, McLeod, \& Cowey, 2007). These subjective measures were able to describe the subjective experience as a function of the experimental manipulations and corresponded mostly with the performance (Del Cul et al., 2007; Jannati \& Di Lollo, 2012; Lau \& Passingham, 2006; Sandberg et al., 2010; Sandberg et al., 2011; Zehetleitner \& Rausch, 2013). Therefore, the subjective measures can be interpreted as reliable measurements of conscious perception.

This one-sided evaluation of subjective and objective measures, with the assumption of a superiority of subjective measures for statements about conscious and unconscious processing, can be doubted. Instead, it has been shown that the comparison of different objective measures allows such statements, too (Schmidt \& Vorberg, 2006). Schmidt and 
Vorberg (2006) referred to three approaches which provided evidence for unconscious processing of a visual stimulus, by juxtaposing a direct and an indirect objective task. A direct measure can be defined as a measure of discrimination, identification or detection performance on alternative stimuli states (for example shape, semantic category), which are part of the instruction (Reingold \& Merikle, 1988). An indirect task captures responses, which were not part of the task definition (Reingold \& Merikle, 1988), for example the response time on a following masking stimulus, whose processing is influenced by the first barely visible stimulus. One of these approaches was referred as a simple dissociation where the direct measure indicate zero performance, whereas an indirect measure show a non-zero value (Schmidt \& Vorberg, 2006). But Schmidt and Vorberg (2006) also emphasized the disadvantages of this approach, since it is based on several assumptions, for example that the direct task measures conscious perception exhaustively and exclusively. Instead, they considered the double dissociation to be more promising, because it requires weaker assumptions. To show a double dissociation, parametric variations of experimental variables are necessary to observe whether the effect of the variation is equal for both, the direct and indirect task. A double dissociation is found, if both tasks are influenced differently by the experimental variation (Schmidt \& Vorberg, 2006). For example, the performance could decline and the response times could rise with increasing temporal delay between the two stimuli. This would speak in favor for the assumption, that both tasks could be attributed to different processing mechanisms. This short excursion is intended to illustrate that the supremacy of subjective over objective data is only a point of view that can certainly be doubted. Even if this work focusses primarily on subjective data, this does not mean that objective data should be neglected or judged as being less meaningful in the study of conscious perception.

All the subjective measurements mentioned above have the communality to differentiate awareness only in a quantitative manner, without taking into account qualitative chances in the appearances of the stimuli across experimental condition. For example the original 4-point PAS ranges from "no experience", "brief glimps", "almost clear experience" to "clear experience" (Ramsøy \& Overgaard, 2004), whereas the endpoint of gradual scales are labeled with "not seen" and "maximal visible" (Del Cul et al., 2007; Sergent \& Dehaene, 2004). Therefore, it is possible that qualitative changes in experiences go unnoticed. Furthermore, no statements about the phenomenology can be made. Gallagher and Sørensen (2006) stated that the use of a rating scale in an experimental task, cannot be regarded as a naturalization of phenomenology. 
In summary, despite the problems, subjective data have always played an important role in capturing conscious experience. Especially since this genuine subjective phenomenon cannot be captured exclusively by objective measures (see section 1.1). But the emphasis on solely qualitative subjective or objective measures can certainly be viewed critically. Nevertheless, even if there are several types of subject measures, after the failure of the first introspective approaches, most of them capture only one-dimensional and quantitative changes in the experience, leaving aside qualitative variations in phenomenological experiences.

\subsection{Subjective experiences in metacontrast masking paradigms}

Masking paradigms allow examining conscious perception in a controlled experimental setting. "Visual masking refers to the reduction of the visibility of one stimulus, called the target, by a spatiotemporally overlapping or contiguous second stimulus, called the mask" (Breitmeyer \& Öğmen, 2006, p. 2). The temporal distance between both stimuli is varied either as stimulus-onset-asynchrony (SOA) or interstimulus interval (ISI). In order to observe the influence of the mask on the perception of the target stimulus, the visibility of the target can be considered as a function of SOA or ISI, the so-called masking function. There are several different kinds of masking, some of them are briefly described, based on the review of Breitmeyer and Öğmen (2006)

Metacontrast masking is a special kind of backward masking, where a target stimulus is followed by a non-overlapping masking stimulus with adjacent contours. For paracontrast masking the same spatial condition have to be met, but the target follows the mask in time, therefore, it is a kind of forward masking. Masking by structure differs by its spatial properties from metacontrast or paracontrast. For masking by structure, the mask spatially overlaps the target and consists of elements that have the same figural characteristic as the target in terms of orientation, curvature and angularity (Breitmeyer \& Öğmen, 2006). This kind of masking has also been termed as pattern masking (Turvey, 1973).

As a particularity of metacontrast compare to other forms of masking non-monotonic, u-shaped Type-B masking functions can be obtained (Breitmeyer \& Öğmen, 2006). The highest visibility is often found at $0 \mathrm{~ms}$ SOA and maximal metacontrast effect at intermediate SOAs (50-100 ms) (Breitmeyer \& Öğmen, 2006). Monotonous Type-A masking functions can also be achieved with metacontrast masking. Type-A masking functions show the largest masking effect at $0 \mathrm{~ms}$ SOA and increased visibility with SOA (Breitmeyer \& Öğmen, 2006). 
The mask-to-target energy ratio determines whether a Type-A or Type-B masking functions is achieved, whereby the energy of a stimulus is defined by its duration, luminance or contrast (Bachmann \& Francis, 2003). The authors concluded that Type-A masking functions result from a low mask-to-target energy ratio and Type-B from a high mask-to-target energy ratio.

In their review Breitmeyer and Öğmen (2006) outlined that the type of the masking function also depended on the task, participants had to perform. Type-B functions were obtained if participants had to respond as fast as possible to the position of the target, with suppressed target brightness or with contour or figural identity tasks. In contrast, simple detection or reaction time task, in which participants only had to distinguish whether or not a target had been presented, did not show accentuated masking functions. Differences in the masking function found in dependence of the tasks were explained with variations of the criterion content (Breitmeyer \& Öğmen, 2006). The criterion content was defined as the stimulus attribute, psychological dimension (Ventura, 1980) or perceptual cue participants based their performance on (Kahneman, 1968). This shows that the exact masking function is related to the aspects of the target, participants are intruded to focus at. Therefore, the dependence of the masking function on the task is a first hint for the richness of the phenomenology in a metacontrast masking paradigm.

In accordance, different aspects of the target were found to vary systematically with SOA. Kahneman (1968) singled out evidence for a varying brightness perception across SOA in metacontrast masking paradigms with flashes of light as targets and mask. It has been shown that the experience of the brightness of the target varies across SOA resulting even in metacontrast suppression, with a phenomenally absent target (Fehrer \& Biederman, 1962; Fehrer \& Raab, 1962; Kahneman, 1967; Weisstein \& Growney, 1969). Even the meaning of metacontrast originates from the phenomena of a contrast reduction of a flash of light by a second flash in an adjacent region of the visual field (Stigler, 1910). Stigler (1910) observed that the perceived luminance of a first flash was reduced by a shortly presented second flash in a way that only the periphery of the first flash was lighted while the rest of the stimulus seemed dark. He called this phenomena "metaphotischer contrast" or simply metacontrast. With black stimuli on a white background, the perception of the dark target varied across SOA with maximum reduction of the black contrast at intermediate SOAs (Breitmeyer et al., 2006; Neumann \& Scharlau, 2007; Werner, 1935). Even a brightness reversal was found under metacontrast masking (Heckenmueller \& Dember, 1965a; Purcell \& Dember, 1968; Stewart, Purcell, \& Pinkham, 2011; Werner, 1935). Another perception which has been reported to vary with SOA was the perception of an apparent motion (Fehrer \& Raab, 1962; 
Kahneman, 1967; Toch, 1956; Weisstein \& Growney, 1969). Also the perceived temporal relationship between target and mask varied with the actual temporal separation. At short SOAs the target seemed to be integrated inside the mask (Eriksen \& Rohrbauch, 1970; Francis \& Cho, 2008; Neumann \& Scharlau, 2007; Reeves, 1982; Scheerer, 1973; Scheerer \& Bongartz, 1973), but at long SOAs a temporal separation between target and mask could be perceived (Francis \& Cho, 2008; Neumann \& Scharlau, 2007; Reeves, 1982). These different target aspects illustrate that the perception of the target does not seem to be uniform. Instead, the perception of the target seems to vary qualitatively across different conditions of a metacontrast masking paradigm.

Individual differences in discrimination performance provided another evidence for the richness of the perception in a metacontrast paradigm. Thus, for identical conditions, participants with Type-A and Type-B masking functions were found (Albrecht \& Mattler, 2012b; Maksimov, Murd, \& Bachmann, 2011). Interindividual differences were explained with different perceptual cues participants use to discriminate the target form (Albrecht \& Mattler, 2012b, 2012a, 2016; Maksimov et al., 2011). Participants who showed Type-B masking functions reported to use a negative afterimage (Albrecht \& Mattler, 2012a). Participants exhibiting Type-A masking functions used a rotation to discriminate the target form, which resulted from shape-incongruent target-mask pairs (Albrecht \& Mattler, 2012a; Maksimov et al., 2011).

In addition, Albrecht and Mattler (2016) provided evidence for independent processes underlying metacontrast masking at short and long SOAs. They assumed that the process underlying metacontrast masking at short SOAs was associated with the perception of an afterimage. They found evidence for two independent processes at long SOAs, associated with the perception of a rotation or a general visibility of a temporally separated target, respectively. Due to differences in the experience of the target and the relation with SOA, metacontrast was stated as a multidimensional phenomenon (Albrecht \& Mattler, 2016; Sackur, 2013).

Altogether, it can be concluded that there are qualitative different subjective experiences in a metacontrast masking paradigm, which may provide an insight into the processes underlying metacontrast masking. But until now, no systematic investigation of the phenomenology in a metacontrast masking paradigm was carried out, which tried to capture the perception without specifying the perceptual categories. Therefore, it is still unknown if and how the experience of the target differs between conditions in a metacontrast masking 
paradigm and how the differences in the perception are related to processes underlying metacontrast masking.

\subsubsection{Theories about the mechanism of metacontrast masking}

There are several different theories about the mechanism of metacontrast masking and it is an ongoing debate which theory is able to explain more properties of metacontrast masking. The theories can be separated into two broadly defined categories, lateral inhibition models (Bridgeman, 1971, 2001; Francis, 1997; Macknik \& Livingstone, 1998; Macknik \& Martinez-Conde, 2004, 2007; Stigler, 1910; Weisstein, 1972; Weisstein \& Growney, 1969) and models differentiating between feedforward and feedback mechanisms (Bachmann \& Kirt, 2013; Bridgeman, 1980; Di Lollo, Enns, \& Rensink, 2000; Fahrenfort, Scholte, \& Lamme, 2007; Kirt \& Bachmann, 2013; Ro, Breitmeyer, Burton, Singhal, \& Lane, 2003; Tapia \& Beck, 2014; Tapia \& Breitmeyer, 2011). These theories are discussed in detail below.

\subsubsection{Theories of lateral inhibition and feedforward processing}

In one of the first studies on metacontrast, it was termed as a retinal phenomenon (Stigler, 1910) Stigler proposed that neighboring cells of the retinal inhibit each other by horizontal connections. The smaller the spatial distance between the two stimuli, the greater the inhibitory influence would have to be. Therefore, the finding that both the magnitude and the form of Type-B masking functions were influenced by the spatial separation of target and mask, was interpreted as evidence of a lateral inhibition mechanism of metacontrast masking (Breitmeyer \& Öğmen, 2006). Breitmeyer and Öğmen reviewed that the masking strength decreased and the peak shifted toward shorter SOAs with increasing distance between both stimuli. This effect was mediated by the eccentricity. For foveal stimuli the influence of the spatial separation was stronger, compared to non-foveal presented stimuli. For non-foveal stimuli metacontrast masking was obtained even at $3^{\circ}$ spatial separation (Growney, Weisstein, \& Cox, 1977), whereas metacontrast effect for foveal stimuli disappeared at a small spatial separation (Stigler, 1910).

However, the localization of metacontrast mechanisms on the retinal level can be excluded, since there is evidence that metacontrast masking can be obtained not only with monoptic but also with dichtoptic presentation, in which target and mask are not presented to the same but to different eyes (Breitmeyer \& Öğmen, 2006). Type-A and Type-B functions 
can be obtained by dichoptic masking (Kahneman, 1968). Macknik and Martinez-Conde (2004) found evidence for monoptic masking effects being located in cells of the lateral geniculate nucleus of the thalamus (LGN) and primary visual cortex (V1), but dichoptic masking was found only in binocular cells of V1. Therefore, at least dichoptic masking effect can be localized in the cortex. In their review Macknik and Martinez-Conde (2007) stated that masking relies solely on feedforward, lateral inhibition effects between the spatiotemporal edges of target and mask. Macknik and Livingstone (1998) attribute a reduction of the transient onset-response of the target or the after-discharge elicited by the offset of the target, as the key mechanisms to produce a decreased target visibility.

Weisstein built one of the first quantitative neural network model, which implemented lateral inhibition as one key components to explain the u-shaped metacontrast masking function (Weisstein, 1968, 1972). Weisstein (1968) proposed that there were two types of neurons, excitatory and inhibitory, whose information converges on a decision neuron. She proposed that the neurons excited by the target have excitatory synapses which converged at the decision neuron, whereas neurons excited by the mask inhibit the decision neuron. In a later works, this physiological implausible asymmetry between different neurons reacting to target and mask stimuli was removed (Weisstein, 1972; Weisstein, Ozog, \& Szoc, 1975). The authors implemented a mutual inhibition between fast and slow responding neuron assemblies. When two neurons with different latencies were stimulated by two visual inputs, the faster reacting neuron inhibited the slower one by horizontal cross inhibitions. Therefore, even an earlier presented stimulus can be inhibited by a following stimulus, because the fast responses of the second stimulus were able to inhibit the slower responses of the preceding stimuli (Weisstein, 1972; Weisstein et al., 1975). These authors used differences in latency to explain u-shaped or monotonic masking function, in dependence of the mask-to-target energy ratio.

Francis (1997) built a quantitative, computational model, capable of simulating most of the properties of metacontrast masking. He defined three mechanisms, which were all based on the principle of lateral inhibition, excitatory feedback, feedforward inhibition and inhibitory feedback. He assumed that the presentation of a stimulus triggers a feedforward signal, which is required for the representation of contours. Based on his model, the neural signal is maintained even after the disappearance of the stimulus by positive excitatory feedback, which decreases continuously over time. The stronger the positive excitatory feedback at higher processing levels, the less it is affected by the inhibitory feedforward signal, linked to the presentation of the mask. 
Based on the literature, Francis (1997) mentioned nine key properties of metacontrast masking, his model was able to replicate. First, u-shaped masking functions were obtained with maximum metacontrast masking at intermediate SOAs (50-100 ms). Second, the shape of the metacontrast function depended on the target luminance, the lower the luminance of the target, the stronger the masking at shorter SOA. Third, with increasing target duration, the detection of the target increased. Fourth, masking effect declined with increasing spatial distance between target and mask. Fifth, with increasing duration of the mask the visibility of the target decreased at short SOAs, resulting in a Type-A masking function with zero visibility at short SOAs, if the mask duration exceeded target duration. Sixth, masking effects increased with the contour of the mask. Seventh, the visibility of the target got better, if a second mask was presented after the first. Eighth, the disinhibition depended on the order and temporal distance of both masks. Ninth, disinhibition decreased with increasing spatial separation between both masks. He explained the first three properties with excitatory feedback, property four to six with feedforward inhibition and the last three with inhibitory feedback. Francis (2007) mentioned that the assumption of a computational feedback should not be confounded with anatomical feedback as it is unclear how it could be implemented in a model.

Similar to Francis model, Bridgeman $(1971,2001)$ formulated another mathematical, lateral inhibition model which distinguished between an excitatory input and a lateral inhibition. Bridgeman (2001) systematically compared his model (Bridgeman, 1971) with that of Francis (1997). He concluded that his model was able to simulate the key properties mentioned by Francis. But, it failed to explain the effects of the duration of target and mask as well as the contour on the masking function. However, Bridgeman (2001) proposed that only his model was able to explain why a shift in the criterion content yielded Type-A functions for tasks with low criterion.

To sum up, most lateral inhibition models were able to simulate or explain the change from $\mathrm{u}$-shaped to monotonic metacontrast functions, in dependence of experimental manipulations. Therefore, they provided a high explanatory power for influences on the masking function that mostly occur at short SOAs.

\subsubsection{Theories of feedback mechanism}

Despite the strength of lateral inhibition models to simulate several findings from metacontrast literature, there was evidence against exclusive feedforward processing as 
assumed by lateral inhibition models. Based on the assumption that metacontrast is not localized at the level of the LGN, Bridgeman (1980) recorded single-cell responses in the striate cortex (area 17) of two macaque monkeys. The monkeys performed a simultaneous brightness discrimination task with two pairs of light targets and masks presented on both sides of the fixation cross. The monkey had to indicate via button press which target mask sequence appeared brighter. For single-cell recording only the SOA was used in which the metacontrast condition was perceived as darker compared to the simultaneously presented target and masks. Differences in correct and incorrect trials were considered for early and late single-cell responses, separately. Trials on which the monkey did not indicate the metacontrast condition as darker were defined as incorrect. For early responses, no difference was found between correct and incorrect trials, but later responses showed a significant enhancement of activity in correct trials. Since the physical stimulus intensity of simultaneous and metacontrast trials was identical, the difference between correct and incorrect trials at late responses was interpreted to correspond not to physical intensity but to perceived brightness. Bridgeman (1980) proposed that the early effect of metacontrast might depend on lateral inhibition, but the late component seemed to be influenced by other mechanisms, which he subsumed under a "cognitive label". He assumed that his results indicated that "visual information enters the primary cortex in an early burst of activity, then is carried by corticocortical connections to other areas, perhaps outside the striate cortex, where it is convolved with other types of (endogenous) information and is then returned to the same striate cortex cells for further processing" (Bridgeman, 1980, p. 361). This first evidence for the influence of reentrant mechanisms on metacontrast masking had impact on several masking theories (Di Lollo et al., 2000; Fahrenfort et al., 2007; Ro et al., 2003; Tapia \& Beck, 2014; Tapia \& Breitmeyer, 2011).

Feedforward and feedback mechanisms of object processing. The adoption of feedback mechanisms contradicted the traditional assumption that visual information is processed exclusively feedforward (Hubel \& Wiesel, 1962). Meanwhile, feedback mechanisms have been integrated into theories of object processing (Bar, 2003; Fenske, Aminoff, Gronau, \& Bar, 2006; Lamme \& Roelfsema, 2000), which served as the basis for several masking models. In both models of Lamme and Roelfsema (2000) as well Bar (Bar, 2003; Fenske et al., 2006), object processing was considered as a two-part process: In a first step a fast but coarse object representation is built up via the dorsal pathway, which ran from V1 via MT to the parietal cortex. This representation is fed back to the ventral pathway which 
ran from V1, via V4 to the temporal cortex, where it facilitates the detailed and slower object processing by activating possible object representations.

These assumptions were embedded in various masking models. In the review of Lamme and Roelfsema (2000) the distinction between conscious and unconscious processing was associated with feedforward and reentrant processing, respectively. The authors assumed that at backward masking paradigms, the feedforward sweep activated by the target remained intact, while the recurrent processing was disrupted by a following mask. This assumption was also supported by electrophysiological results of Fahrenfort et al. (2007) who examined the effect of a backward pattern mask on the processing of second-order, orientation defined targets. Fahrenfort et al. (2007) concluded that masking disrupted reentrant process, while the early feedforward sweep remained intact, but was not sufficient to generate a conscious perception of the target. A similar interpretation was drawn in a former study of Lamme, Zipser, and Spekreijse (2002). Single cell recording from V1 of awake macaque monkey was obtained, while the animals performed a figure detection task under backward pattern masking, with second-order texture defined stimuli. At the SOAs where the animals could not recognize the figure, the activity that reflected figure-ground segregation and that was associated with reentrant feedback, was affected by the mask. But the low-level, earlier activity, which reflected orientation differences of figure and background, was not suppressed by the mask.

To sum up, there is evidence from electrophysiology studies, that backward masking disrupts feedback loops, necessary for consciousness object processing, whereas the first feedforward sweep is left intact. Both, the assumption of two processing paths and the hypothesis that masking effects are exclusively due to an interruption of reentrant processing, have been adopted in various theories of metacontrast masking. This group of masking theories can be distinguished from those that assume exclusively feedforward processing to explain metacontrast masking effects. In the following, two masking models requiring feedforward and feedback mechanisms are presented in detail.

Object Substitution Masking. Di Lollo et al. (2000) assumed that visual processing took place in several steps. First, a feedforward sweep results in an initial, incomplete and illdefined representation of the object. To solve the ambiguity of the representation reentrant processing is necessary, allowing the visual system to compare the coarse high-level information with the more precise information that was slowly processed through low-level areas in a second step. The mask's impairment of the target visibility was explained by a mismatch between the initial information of the target which was fed back to lower visual 
areas at the same time the feedforward information of the mask arrived. This mismatch occurred in masking conditions where the target was briefly followed by a mask, at a time point when the processing of the target was not completed. As a consequence, the target was not be perceived. Di Lollo et al. (2000) termed this process object substitution.

The authors based their assumptions on so-called common-onset masking paradigms, where several Landolt rings as targets were presented simultaneously, with one ring surrounded by a mask. Targets and mask had the same onset, but after $10 \mathrm{~ms}$ the targets disappeared, leaving just the mask on the screen for a variable amount of time. The task was to indicate the position of the gap of the target, which was surrounded by the mask. If the mask consisted only of four small dots, the paradigm was termed as four-dot masking. Di Lollo et al. (2000) listed several results as evidence for object substitution: the increase of masking strength with increasing mask duration and set size (Experiment 1), the decrease of masking by a pop-out effect of the target (Experiment 5) or with a spatial cue, predicting the location of the target at the multi-element display (Experiment 6). The authors assumed that all of the three manipulations had an influence on iterations needed to compare the coarse perceptual hypothesis, built on the first step of processing, via reentrant loops with the currently dominant precise information processed at low-level areas. For example, with increasing set size more iterations were required, increasing the risk that the target information vanished before the comparison was completed.

Enns and Di Lollo (2000) adopted Bridgeman's (1980) assumption that two components were responsible for masking effects. As low-level component they assumed contour interactions to be responsible for masking effects at short SOAs, whereas the mechanism of object substitution was regarded as the second and high-level component of masking. Evidence for two different components resulted from the absent of an effect of the set size on the masking strength in dark-adapted viewing conditions, which they assumed to impact only low-level processes (Di Lollo et al., 2000, Experiment 2). In addition, object substitution was not influenced by the spatial proximity of target and mask, which is known to affect masking based on inhibitory contour interactions (Di Lollo et al., 2000, Experiment 3). Di Lollo et al. (2000) proposed that masking effects obtained with a mask which consisted of four little dots cannot be explained by inhibitory contour interactions, since contour interactions depend on the size of the masking contour (Experiment 3,4). Therefore, with four-dot masking paradigms instead of contour interactions the mechanisms of object substitution was regarded to be responsible for masking effects. In addition, the mechanism of 
object substitution was extended to other kinds of masking, inter alia metacontrast masking (Di Lollo et al., 2000; Enns \& Di Lollo, 2000).

RECOD model of masking. There are several different versions of the retino-cortical dynamics (RECOD) model. The original sustained-transient channel inhibition model of masking (Breitmeyer \& Ganz, 1976) was formulated exclusively as a feedforward model. But a further development and mathematically formalization of RECOD model also included feedback mechanisms (Öğmen, Breitmeyer, \& Melvin, 2003).

The RECOD model was expanded by the differentiation between sustained activity within the ventral pathway and transient activity within the dorsal pathway (Öğmen et al., 2003; Tapia \& Breitmeyer, 2011). Both pathways, the faster magnocellular dominated dorsal and the slower parvocellular dominated ventral pathway were assumed to process different stimulus features in a bottom-up manner (Tapia \& Breitmeyer, 2011). Several assumptions of Bars object processing model (Bar, 2003; Fenske et al., 2006) have been incorporated into the RECOD model of Tapia and Breitmeyer (2011). First, they assumed that the fast but coarse processed information within the dorsal pathway is projected from the PFC in a top-down manner to the IT. At the same time the slow, detailed and feedforward processed information of the ventral path reaches the IT. Second, the RECOD model was extended by reentrant activity within the ventral pathway. Tapia and Breitmeyer assumed that feedback from the dorsal pathway triggers a reentrant processing within the ventral pathway, which amplifies signals at a low-level and increases the selectivity of neurons at higher level. Tapia and Breitmeyer (2011) assumed that the strength of magnocellular generated activity of the dorsal path modulates the strength of the top-down feedback from the PFC to the IT of the ventral pathway. Therefore, the magnocellular activity is directly related to the reentrant feedback at the ventral pathway, which is assumed to be responsible for conscious perception. At masking conditions they assumed that only the feedforward sweep within the ventral pathway is left intact, which is not suffice to produce conscious perception, but modulates the unconscious perception. Tapia and Breitmeyer concluded that the reentrant processing is a central component for conscious vision and is suppressed by metacontrast masking, whereas the feedforward sweep is left intact.

One strength of the RECOD model was to take into account differences in the masking effect according to the criterion content. First, u-shaped masking functions were only obtained when target's surface properties were judged, whereas reports of the present of the target revealed no accentuated masking functions (Breitmeyer \& Öğmen, 2006). In accordance, the mathematical formalization of the RECOD model was able to simulate a u-shaped function 
for a brightness rating task, whereas no metacontrast masking effects were found with choice reaction time tasks (Öğmen et al., 2003). Breitmeyer et al. (2006) considered metacontrast masking not as a unitary phenomenon, but proposed independent processing mechanisms for different object features, explicitly surface and contour processing along the parvocellular pathway. They proposed an inter-channel inhibition between the magno-dominated dorsal and parvo-dominated ventral path. A briefly presented target was assumed to exhibit a fast transient activation, followed by a slower sustained contour processing and last by a sustained surface, respectively brightness processing. If and how much the sustained contour or surface processing of the target is disturbed by the transient activity of the mask, depends on the SOA. At short SOAs the transient activity elicited by the mask initially disturbs the rapid contour processing of the target within the ventral pathway. The slightly slower surface processing of the target can only be disrupted by a mask following the target at intermediate SOAs.

\subsubsection{Summary}

There are several different models for backward masking, respectively metacontrast masking and it is still an open debate which is more appropriate to describe masking effects. Especially initial models only assumed feedforward processing and one general mechanism, namely lateral inhibition (Bridgeman, 1971, 2001; Francis, 1997; Macknik \& Livingstone, 1998; Macknik \& Martinez-Conde, 2004, 2007; Stigler, 1910; Weisstein, 1972). Later models integrated electrophysiological findings of object processing (Bar, 2003; Fenske et al., 2006; for review see, Lamme \& Roelfsema, 2000) by proposing feedforward and feedback, respectively reentrant mechanism (Bachmann \& Kirt, 2013; Bridgeman, 1980; Di Lollo et al., 2000; Fahrenfort et al., 2007; Kirt \& Bachmann, 2013; Ro et al., 2003; Tapia \& Beck, 2014; Tapia \& Breitmeyer, 2011). Some of these models included different mechanisms at short and long SOAs. At short SOAs, low-level local contour interactions were proposed, at longer SOAs a high-level component, which was associated with the disruption of reentrant processing and object substitution masking (Bachmann, 2015; Bridgeman, 1980; Di Lollo et al., 2000; Enns \& Di Lollo, 2000). Models which proposed feedback mechanisms, implemented findings of different processing speeds for different object features (Bachmann \& Kirt, 2013; Breitmeyer et al., 2006; Kirt \& Bachmann, 2013). Thereby, these models were able to explain differences in the phenomenology of the target in dependence of the temporal relation between target and mask. 
1 | Motivation to consider phenomenology in a metacontrast masking paradigm

\subsection{Motivation to consider phenomenology in a metacontrast masking paradigm}

The last part of the introduction summarizes why it is worth to consider the phenomenology in a metacontrast masking paradigm and which question can be answered by such an approach.

First, an approach to naturalize phenomenology is presented which is based on a combination of neurophenomenological and heterophenomenological methods (Gallagher \& Sørensen, 2006). This approach was assumed to fulfill the requirements of a phenomenological methodology by measuring subjective experiences reliable. In addition, it allows an appropriate incorporation of phenomenological requirements into a scientific framework, by observing commonalities between individual experiences and their relation to experimental parameters. Masking paradigms are suitable for phenomenological examinations. They ensure controlled experimental settings to evaluate phenomenological descriptions systematically. Parametric manipulations allow a validation of the phenomenological descriptions, by considering variations in the experiences as a function of the manipulations. Even simple visual stimuli can be masked. Since the perceptual dimensions of simple stimuli are more limited than those of complex visual stimuli or scenes, interindividual similarities in perception are more likely to be identified. This helps to overcome the difficulties of previous phenomenological studies.

Second, the phenomenology of metacontrast masking has not been investigated systematically so far. Consequently, little is known about the appearance of the target in a metacontrast masking paradigm. Instead, there are several one-dimensional scales that measure subjective experiences exclusively quantitative (e.g. Ramsøy \& Overgaard, 2004; Sandberg et al., 2010; Szczepanowski et al., 2013; Zehetleitner \& Rausch, 2013). These onedimensional scales are based on the assumption that the perceptual experience of the target stays constant across conditions. Therefore, these scales imply that the target can always be described in the same dimensions of size, form, hue, color, orientation and so on. However, there are also results that speak against the use of one-dimensional scales. These results indicate differences in the appearance of the target, depending on experimental variations, such as the temporal or spatial relationship between target and mask. There is evidence for differences in the contrast of the target (e.g. Fehrer \& Biederman, 1962; Fehrer \& Raab, 1962; Stewart et al., 2011; Werner, 1935), the perceived temporal distance between target and mask (e.g. Eriksen \& Rohrbauch, 1970; Francis \& Cho, 2008; Neumann \& Scharlau, 2007; Reeves, 1982 ) and the perception of a motion (e.g. Fehrer \& Raab, 1962; Kahneman, 1967; Weisstein 
$\&$ Growney, 1969). These results support the assumption that the subjective experience of the target is not constant across conditions, but varies systematically at metacontrast masking paradigms (Kahneman, 1968). Therefore, only the detachment of subjective scales, which already prescribe the appearance of the target, allows to comprehensively grasp the phenomenology of metacontrast masking.

Third, it has been shown that differences in the subjective experience provide insights into the mechanisms of metacontrast masking, by linking differences in the experiences with different processes (Albrecht \& Mattler, 2016; Neumann \& Scharlau, 2007; Reeves, 1982; Sackur, 2013). Furthermore, there is a controversy about mechanisms underlying metacontrast masking. Some authors assume lateral inhibition as a key mechanism of metacontrast masking (Bridgeman, 1971, 2001; Francis, 1997; Macknik \& Livingstone, 1998; Macknik \& MartinezConde, 2004, 2007; Stigler, 1910; Weisstein, 1972), others suggest higher masking mechanisms such as object substitution, which are related to feedback or reentrant processing (Bachmann, 2015; Bridgeman, 1980; Di Lollo et al., 2000; Enns \& Di Lollo, 2000). Even if there are many theories about the mechanisms of metacontrast masking, only a few take into account differences in the experience of the target. It is doubtful whether theories that do not explain differences in the appearance of the target or consider only one general masking mechanism, are sufficient for metacontrast masking, which has been defined as a multidimensional phenomenon (Sackur, 2013).

This thesis has the aim to enrich the understanding of metacontrast by considering qualitative differences in the experiences of the masked target. Variations in the criterion content across SOA are examined, to provide evidence for the multidimensionality assumption of metacontrast masking. Further, the relationship between phenomenological and objective measures is considered. Finally, phenomenological data as well as electrophysiological findings should enrich the understanding of the processes underlying metacontrast masking. 


\section{General Methods}

\subsection{Stimuli}

The target stimuli were filled squares and diamonds with a diameter of $1.5^{\circ}$ visual angle. The mask had a square- or diamond-shaped outer contour with a diameter of $2.6^{\circ}$ visual angle and a star-shaped inner contour that neatly surrounded the contours of the target, leaving space for one pixel $\left(0.02^{\circ}\right.$ visual angle) (Figure $\left.2.1 \mathrm{~A}\right)$. All stimuli were presented in black $\left(0.03 \mathrm{~cd} / \mathrm{m}^{2}\right)$ on a light grey background $\left(72.3 \mathrm{~cd} / \mathrm{m}^{2}\right)$ in the center of the screen of a CRT-Monitor (ViewSonic GF90-B, refresh-rate $85 \mathrm{~Hz}$ ). Target and mask durations were $24 \mathrm{~ms}$ and $106 \mathrm{~ms}$, respectively. The SOA between target and mask varied between 24, 36, $48,60,72$ and $84 \mathrm{~ms}$. In half of the trials, target and mask were congruent, i.e. both stimuli were either squares or diamonds. In the other half of the trials target and mask were incongruent, i.e. one stimulus was a diamond and the other on a square or vice versa.

\subsection{Procedure and Design}

The experiments took place in a dimly lit room with participants' heads resting on a chin rest $100 \mathrm{~cm}$ from the screen. The trial sequence was for all experiments: Each trial started with a fixation cross for 750 milliseconds followed by the target for $24 \mathrm{~ms}$ and the mask for $106 \mathrm{~ms}$ (Figure 2.1B). After a response was given the next trial started after a random inter-trial interval between 750 and $1250 \mathrm{~ms}$. The way in which an answer was given varied between the different experiments. Participants were instructed to fixate on the cross over the entire trial.

Unless stated otherwise, independent variables Target (square vs. diamond), Mask (square vs. diamond) and SOA $(24,36,48,60,72,84 \mathrm{~ms}$ ) varied pseudo-randomly within each block so that each of the 24 combinations occurred equally often. In none of the experiments an auditory feedback was given. 
A
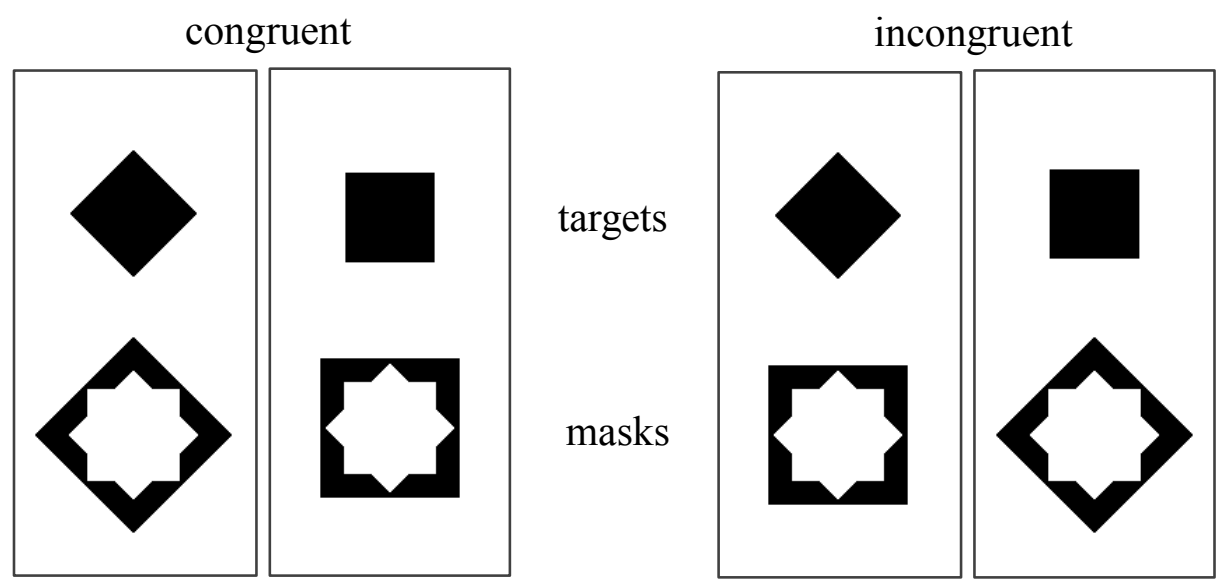

B

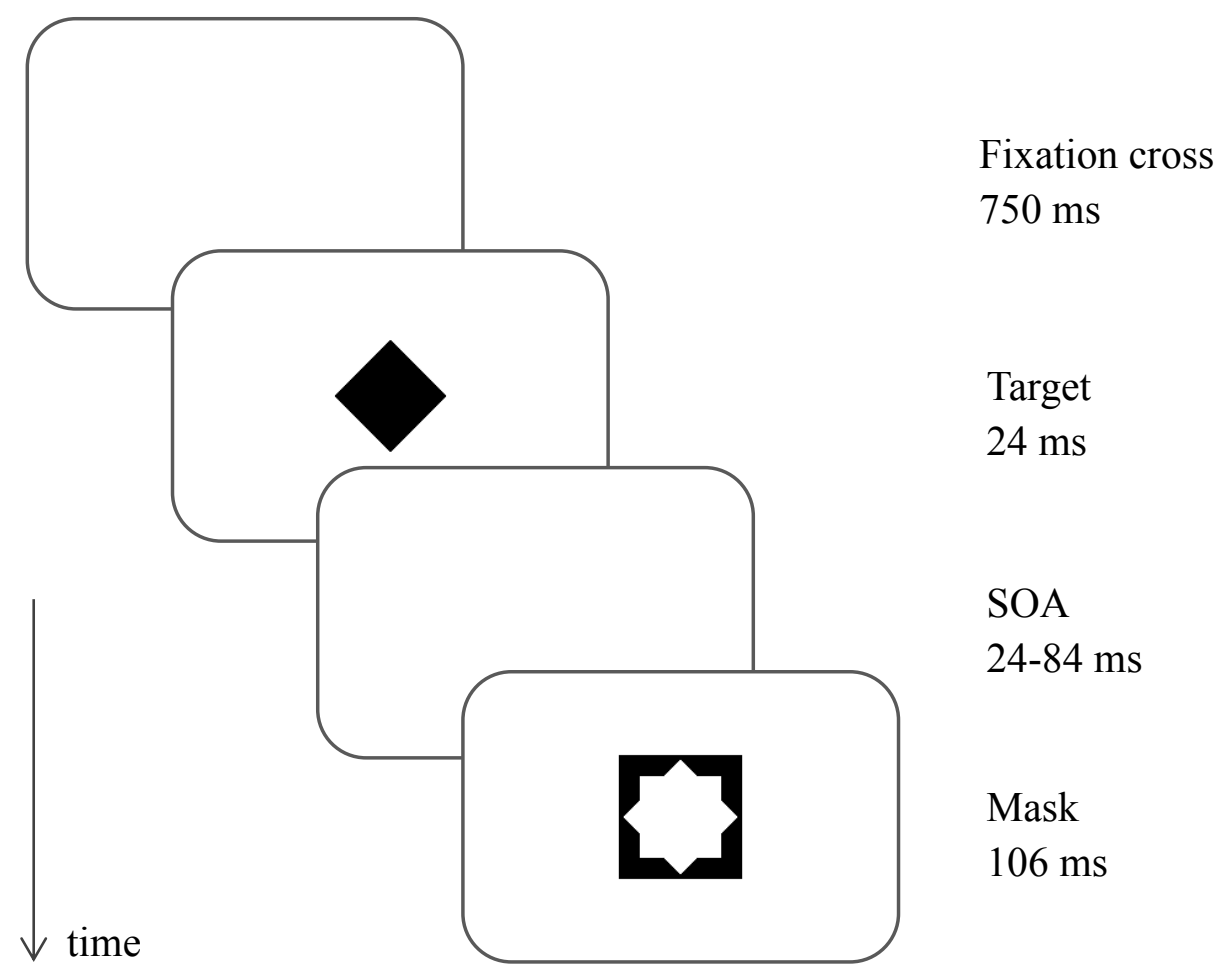

Figure 2.1. A Square- and diamond-shaped target and mask stimuli, illustrating congruent and incongruent target-mask pairs. B Trial sequence, exemplary for an incongruent target-mask pair. 
3 | Visual perception is rich. Phenomenological evidence from metacontrast masking

\section{Visual perception is rich. Phenomenological evidence from metacontrast masking}

This chapter was written in collaboration with Dr. Thorsten Albrecht.

\subsection{Abstract}

A metacontrast masking paradigm was used to provide evidence for the richness and diversity of our visual experience. Square- and diamond-shaped targets and masks were used, resulting in form congruent and incongruent trials, which were presented randomized with varying SOA. In Experiment 1 on each trial naive participants were asked to report their perception of target and mask. After an intense training, seven different target aspects were identified to be unique perceptions in a metacontrast masking paradigm. The target aspects included the temporal distance between both stimuli, the perceived contrast of the target and motion perceptions resulting from the interplay of target and mask. These perceptions were spontaneously reported and showed distinct time courses across SOA. In Experiment 2, participants were trained to perceive each target aspect. The relationship of each target aspect with SOA and Congruency found in Experiment 1 could be replicated. The discrimination performance captured in a last session depicted individual different masking function with a decreasing performance across SOA on average. Each perception yielded a unique relationship with discrimination performance. However, on average despite rich subjective experiences, low discrimination performance was found, especially at long SOAs. These results suggest that the perception of the target is not constant in a metacontrast masking. Instead, it seems to vary on several perceptual dimensions across conditions. This challenges the usage of one-dimensional subjective or objective measures to state about the awareness of the target. 


\subsection{Introduction of Experiment 1a and 1b}

Usually we subjectively have the impression of a detailed representation of the whole visual scene but phenomena like change blindness and inattentional blindness suggest that even considerable changes in a scene may remain unnoticed (Irwin, 1991; O'Regan \& Noë, 2001). These findings are often considered as evidence that only objects within the focus of attention are represented in rich detail and that the world outside the focus of attention is only sparsely represented, not represented at all or represented in so called ensemble statistics, summarizing multiple objects into an average representation (Cohen, Dennett, \& Kanwisher, 2016; Kouider, Gardelle, Sackur, \& Dupoux, 2010). This discrepancy between our subjective impression of a rich perception and the objective evidence of a sparse representation is taken as evidence for the fallibility of introspective methods that were developed in the beginning of the $20^{\text {th }}$ century. One objection to this view is that the richness of perception is systematically underestimated by restricting participants' reports to coarse and high-level object centered categories, for example, seen vs. not seen; living vs. non-living; square vs. diamond (Haun, Tononi, Koch, \& Tsuchiya, 2017). Usually, participants are forced to choose one of several predefined response alternatives to report the identity of specific stimuli. This traditional approach in experimental psychology lacks the possibility to capture information about lowlevel perceptual experiences such as the perception of individual target features, for example impressions of contrasts or motion, which may contribute substantially to the richness of perception. To investigate the mechanisms that lead to phenomenological experience of visual stimuli and their neuronal basis it is therefore necessary to measure these low-level experiences (Albrecht \& Mattler, 2012a; Fei-Fei, Iyer, Koch, \& Perona, 2007; Spillmann, 2009).

In recent years the importance of subjective measures in consciousness research has gained renewed interest (Chalmers, 1995; Searle, 2000) and methodologies to naturalize Phenomenology have been proposed with the aim to systematically describe subjective experiences within in a scientific framework (Dennett, 1991, 2003; Gallagher \& Sørensen, 2006; Lutz et al., 2002; Overgaard, 2004; Varela, 1996; Varela \& Shear, 1999): First, commonalities in the descriptions across participants, have to be traced back to differences in the experimental manipulation. Second, parametrical experiments allow considering whether the perceptions vary with the conditions. Based on this it can be concluded whether the perceptions depend on stimulus conditions or be rather trial-by-trial fluctuation in the 
3 | Visual perception is rich. Phenomenological evidence from metacontrast masking

perceptions not related to the manipulations. Third, relating subjective and objective data validates subjective reports as reliable perceptual cues to perform the objective task.

In the present study we applied this approach in the context of metacontrast masking and provide evidence that even the perception of simple geometric figures in a starkly reduced stimulation conditions yield a complex and rich pattern of experiences. Metacontrast masking occurs when a target stimulus is followed by a masking stimulus, whose contours fit neatly around the contours of the target stimulus (for review see Breitmeyer \& Öğmen, 2006). One crucial determinant for the visibility of the target is the stimulus onset asynchrony (SOA) between target and mask leading to increasing (Type-A) or u-shaped masking functions across SOA (Type-B) - depending on the exact stimulation parameters. Due to the requirement of contour contiguity between target and mask metacontrast is in almost all cases limited to simple stimuli with clearly defined contours.

Common methods to measure the awareness of a target stimulus in such paradigms encompass different kinds of objective and subjective measures. Objective measures refer to the correct detection, identification or discrimination of the target stimulus (Breitmeyer \& Öğmen, 2006). Subjective measures refer to the clearness of the perception of a stimulus (Del Cul et al., 2007; Overgaard, Rote, Mouridsen, \& Ramsøy, 2006; Sandberg et al., 2010; Sandberg et al., 2011; Schwiedrzik et al., 2011; Sergent \& Dehaene, 2004), to metacognitive judgments about one's own performance (Szczepanowski et al., 2013; e.g., the confidence in an objective task Szczepanowski \& Pessoa, 2007; Zehetleitner \& Rausch, 2013) or to more indirect measures that capture the amount of money the participants are willing to bet on their decision in the objective task (post decision wagering, Dienes \& Seth, 2010; Persaud et al., 2007; Sandberg et al., 2010). All have in common that they restrict the reports to predefined categories (correct vs. incorrect, seen vs. not seen), to a single dimension (for example, magnitude of contrast) or to global measures like the "clearness" of a stimulus. This restriction is surprising because it is widely acknowledged that the phenomenological experience of a target stimulus differs widely across experimental conditions (Jannati \& Di Lollo, 2012; Kahneman, 1968; Koivisto \& Revonsuo, 2008; Sackur, 2013). Due to the variety of phenomenological experiences the criterion content, i.e. the perceptual cue or psychological dimension on which the decision in an objective task is based, may differ between conditions (Kahneman, 1968) as well as participants (Albrecht, Klapötke, \& Mattler, 2010; Albrecht \& Mattler, 2012b, 2012a, 2016). Thus, although the objective performance may be the same in two different SOAs, the appearance and therefore the criterion content may differ substantially between both SOAs, which makes it difficult to compare differences 
between subjective and objective measures across conditions (Jannati \& Di Lollo, 2012). Sackur (2013) employed multidimensional scaling on similarity judgments to infer the perceptual space of metacontrast and found that it unfolded in three dimensions. The first dimension was correlated with SOA. The two other dimensions were associated with the descending and ascending flank of the metacontrast masking function, respectively. Because the second and third dimension of the perceptual space reflected different parts of the metacontrast function, Sackur inferred that the visibility under decreasing integration was perceptually distinct from the visibility under increasing segregation. Taking together these results suggest that the subjective appearance of a target differs qualitatively across experimental conditions in a metacontrast masking paradigm. Consequently, it is doubtful if one-dimensional subjective measures suffice to exhaustively capture subjective awareness in a metacontrast masking paradigm.

\subsubsection{Phenomenology in metacontrast masking}

Already Werner (1935) stated that the appearance of a target in metacontrast may vary from trial to trial despite identical stimulation parameters. But to our knowledge, the phenomenology of the target in a metacontrast paradigm has never been systematically investigated. Nevertheless, the literature on metacontrast yields many notions about the appearance of the target related to (1) the perceived contrast of the target, (2) the perceived temporal order of target and mask and (3) apparent motion:

First, the mask affects the perceived contrast of the target (e.g., Breitmeyer et al., 2006; Kahneman, 1967; Neumann \& Scharlau, 2007; Werner, 1935). For instance, Breitmeyer et al. (2006) asked participants to adjust the contrast of a test patch to the perceived contrast of a target disc that was masked by metacontrast with varying SOAs. The results showed a u-shaped function with high contrast at short and long SOAs and a low contrast at intermediate SOAs. Similar results have been shown when participants rated the perceived contrast of the target directly on a Likert-scale (Neumann \& Scharlau, 2007). At intermediate SOAs of 50-100 ms this metacontrast suppression can reduce the perceived contrast to the background level, i.e. so that the target is not experienced at all. This has been shown with simple light flashes as target and mask (Alpern, 1953; Fehrer \& Raab, 1962; Kahneman, 1967; Schiller \& Smith, 1966; Weisstein \& Growney, 1969) as well as with contour defined stimuli of either polarity (Breitmeyer, Tapia, Kafaligonul, \& Öğmen, 2008; Stewart et al., 2011). Beside the reduction of the perceived target contrast, several studies 
3 | Visual perception is rich. Phenomenological evidence from metacontrast masking

have reported a reversal of the perceived contrast, i.e. a dark target stimulus on light background is perceived as brighter than the background (Heckenmueller \& Dember, 1965b; Purcell \& Dember, 1968; Stewart et al., 2011; Werner, 1935). For instance, Werner (1935) used a black target disc and a black ring as metacontrast mask and reported that on $7 \%$ of all cases the inner field of the ring was much whiter than the grey background. More recently, Stewart et al. (2011) provided further evidence for a brightness reversal: They presented a small black target disk to the left or to the right of the fixation cross followed by two ringshaped metacontrast masks on either side of the fixation cross. Participants indicated the side on which the target disc had been presented. Results showed that at a SOA of $20 \mathrm{~ms}$ participants performed lower than chance level, i.e. they systematically chose the wrong side suggesting that they perceived the target side as brighter than the non-target side. In sum, we assume at least three contrast related perceptual categories with different time courses across SOA: The perception of a Dark Target should follow a u-shaped function across SOA, the precept of No Target should follow an inverted u-shaped function and a Bright Target should be perceived only at short SOAs.

Second, there is ample evidence that metacontrast masking affects the perceived temporal order of target and mask. At short SOAs target and mask have been reported to occur simultaneously, i.e. as temporally integrated percept of a target within the inner contours of the metacontrast mask, at long SOAs target and mask have been reported as two successive events (Neumann \& Scharlau, 2007; Reeves, 1982). This had led to the hypothesis that a successful temporal integration of target and mask determines the visibility of the target at short SOAs (Eriksen \& Rohrbauch, 1970; Scheerer, 1973; Scheerer \& Bongartz, 1973), in the case of metacontrast masking (Francis \& Cho, 2008), whereas at long SOAs a successful segregation of target and mask determines the visibility of the target (Eriksen \& Rohrbauch, 1970; Francis \& Cho, 2008; Jannati \& Di Lollo, 2012; Neumann \& Scharlau, 2007; Reeves, 1982). Therefore, two time-related perceptual categories can be distinguished: An integrated percept (Target inside Mask) with a decreasing trend from short to long SOAs and a segregated percept (Target before Mask) with an increasing trend from short to long SOAs.

Third, motion related percepts have been frequently observed in metacontrast paradigms, probably due to the high similarity between masking and apparent motion paradigms: Depending on optimal spatiotemporal parameters two successive stimuli induce the perception of motion (Wertheimer, 1912). For instance, if a central target bar is masked by two flanking bars an outward motion from the center to the periphery can be seen at SOAs of 50-100 ms (Hogben \& Di Lollo, 1984; Toch, 1956). This effect is strongest within a SOA 
range that is optimal for metacontrast suppression. Accordingly, motion can also be perceived when the target is phenomenologically absent (Fehrer \& Raab, 1962; Kahneman, 1967; Weisstein \& Growney, 1969). Further evidence comes from studies that manipulated the shape-congruency between target and mask (see Figure 2.1A in General Methods for example stimuli). At intermediate to long SOAs participants reported to perceive a rotational motion but only on incongruent trials, i.e. when target and mask differed in shape (Albrecht \& Mattler, 2012b, 2012a; Ansorge, Becker, \& Breitmeyer, 2009; Ansorge, Breitmeyer, \& Becker, 2007; Maksimov et al., 2011). The impression of rotation results from the short stimulus presentation, so that in case of incongruent target mask pairs it appears as if the target would turn into the mask. Albrecht and Mattler (2012a) measured the subjective experiences of motion percepts. Within the same paradigm motion percepts of a rotation as well as an enlargement similar to the outward motion occurred. Albrecht and Mattler (2012a) assumed that motion results from the interaction between target and mask, although the SOA effect remained unclear in their study. Unfortunately, they did not differentiate between both motion percepts in their analysis, but they speculated that expanding motion and rotational motion possibly could occur predominantly at short and long SOAs, respectively, which would explain the lack of SOA effects in their study. In addition, they showed that participants that performed high at long SOAs in an objective target discrimination task (1) more often reported a motion percept and (2) more often reported to use this percept to discriminate the target shape. This relation between individual differences in introspective reports and objective data suggests that at long SOAs (60-84 ms) a motion process may affect metacontrast masking. Taken together, we assume two different motion related perceptual categories: Rotation should occur exclusively on incongruent trials showing an increasing trend with SOA. Assuming that both motion percepts result from apparent motion between target and mask, Expansion should either follow an increasing or u-shaped trend with SOA.

To sum up, perceptions of seven different aspects of the target have been reported in the metacontrast literature, which were associated with the temporal or spatial relationship between target and mask, particularly with SOA and/or Congruency. In addition, there is some evidence that individual differences in subjective reports relate to individual differences in an objective task. But most studies suffer from two severe limitations because they (1) focused only on one or two different percepts and (2) all of the mentioned studies defined the criterion content participants had to focus on. Therefore, it has never been investigated systematical what participants spontaneously perceive, if nothing is prescribed. Consequently, very little is known about the richness of phenomenology in a metacontrast paradigm. 
3 | Visual perception is rich. Phenomenological evidence from metacontrast masking

Following the line of Haun et al. (2017) we hypothesize that a fine-grained analysis of phenomenological experiences reveals a rich phenomenal experience even for simple geometric figures and under conditions of reduced awareness of visual masking. Our approach to measure the phenomenology in a metacontrast paradigm is based on spontaneous reports of visual experiences across a variety of experimental conditions. This allows us to directly map the perceptual space of conscious visual experience under conditions of reduced awareness and to investigate the richness in this starkly reduced stimulation. If different perceptual categories, representing different aspects of the target vary continuously with experimental manipulations in a metacontrast masking paradigm, this would validate our perception as rich and detailed representation of the environment.

\subsubsection{Experiment 1a and 1b}

We ran two phenomenological experiments to investigate (1) if naive participants are able to describe their visual experience of a metacontrast sequence, (2) if these descriptions are related to perceptual categories based on the literature and (3) if we can identify distinct time courses across SOA for these perceptual categories. These findings would provide direct evidence that the spatiotemporal relation between target and mask determines the quality of the visual experience on several distinct dimensions.

In particular, reports of a Target inside Mask should show a decreasing function of SOA (1), reports of a Target before Mask should follow an increasing function of SOA (2), reports of a Dark Target should follow a u-shaped function of SOA (3), reports of a Bright Target should be reported mostly at short SOAs (4), reports of No Target experience should follow an inversed u-shape function (5), Rotation should be reported increasingly with SOA but only on incongruent trials (6), reports of an Expansion should either follow an inverted ushaped function or decrease with SOA (7).

\subsection{Methods}

Participants. A group of fifteen students ( 9 female, 6 male; ages between 20 and 29 years, $M=22.5$ years, $S D=3.1$ years) participated in Experiment 1a. Two participants were excluded due to poor compliance with the instruction. A second group of twenty-four students (17 female, 7 male; ages between 19 and 33 years, $M=22.7, S D=3.4$ years) took part in Experiment 1b. Four participants were excluded due to technical problems $(n=2)$ and due to poor compliance with the instruction $(n=2)$. All participants were from Georg-August 
University Goettingen, had normal or corrected-to-normal vision and received monetary reward. All participants were naive with regard to the aim of the study and never had participated in a metacontrast masking experiment before. The study was approved by the local ethics committee of the Georg-Elias-Müller-Institute of Psychology, Georg-AugustUniversity of Goettingen and all experimental procedures are in accordance with the Declaration of Helsinki.

Task. Both Experiments comprised 5 sessions that lasted between 60 and 90 minutes. In the Training Phase (Sessions 1-3) participants were trained to verbally report their subjective visual experience of the presented stimuli as detailed as possible on every trial. It was heavily stressed that the task was to report the whole visual experience of the stimuli and not only to identify the shape of the target. In Experiment 1a the instruction read "On each trial, please describe your visual experience of target and mask, even if the target can hardly be seen. Do not just report the shape of the target stimulus." In Experiment $1 \mathrm{~b}$ the instruction was changed to "On each trial, please describe your visual experience of the target, even if it can hardly be seen. Do not just report the shape of the target stimulus." By focusing the instruction on the target stimulus we sought to avoid verbal descriptions of the mask only. Participants that were not able to report more than the shape of the target were excluded after the training phase ( $n=2$ in each Experiment, see above). At the end of the training phase participants compiled a list of their most common visual experiences and gave each list item a concise but arbitrary label (for example mask, spot, star, continuum) so that each participant had an individual collection of descriptions of several subjective experiences. In the Test Phase (Sessions 4-5) participants categorized each trial according to their individually collected descriptions of subjective experiences.

Stimuli, Procedure and Design. Stimuli, trial sequence and design were identical to the description in General Methods (Figure 2.1), with the following particularities: After the presentation of the stimuli participants had unlimited time to give a verbally response. In Session 1-3 they described their visual experience and in Session 4-5 they named one of their experiences of their individual collected descriptions. The reports were recorded to an audio file on hard-disk using a boundary layer microphone placed in front of the monitor. Participants ended each trial by pressing the Enter key.

Training Phase (Sessions 1-3). The first session started with 6 warm-up blocks with 4 trials each, so every possible target mask combination once per block. The stimulus combinations varied pseudo-randomly within each block. The SOA was presented blockwise and varied pseudo-randomly between these warm-up blocks. Within the warm-up blocks 
participants did not gave any verbal reports, rather they were instructed to carefully observe the stimulus sequence and reflect on their visual experience. After the warm-up blocks, 3 blocks with 24 trials each followed, in which participants gave detailed verbal reports about their visual experience. If unsure about their experience participants could repeat each trial by pressing the space key until they felt confident to describe the percept ${ }^{1}$. After every 5 to 10 minutes participants were to take a short break, in which the experimenter repeated the instruction in a standardized way. If the three experimental blocks were not finished within one hour the measurement was terminated. At the end of the session participants described their most common visual experiences in detail and additionally drew a sketch for visualization.

Sessions 2 and 3 were identical to Session 1, except that the warm-up blocks were omitted and each trial could be repeated only twice and only in the first block. In Session 3 the debriefing continued with the request to collect and name all most common visual experiences of the first three sessions that were sufficient to describe the whole visual experience of target and mask of the particular participant. This collection was used in two additional blocks with 24 trials each. On each trial participants named the perceived visual experience of their collection. Only one description of the collection could be named on each trial. After each of the two blocks, participants were allowed to modify their collection. The average number of trials performed at the trainings phase was $245.69(S D=39.62)$ for Experiment 1a. In Experiment $1 \mathrm{~b}$ each participant performed 264 trials at the trainings phase.

Test Phase (Sessions 4-5). Both sessions consisted of one warm-up block with 24 trials, which was excluded from the analyses and seven experimental blocks with 48 trials each. On each trial participants named the perceived visual experience of their collection and pressed the Enter key to start the next trial. During the warm-up block the experimenter stayed in the room and the participants could repeat each trial twice. In the experimental blocks trials could not be repeated. At the end of both sessions participants received a careful debriefing identical to the training sessions. Altogether, the test phase comprised of 672 experimental trials, 56 trials per condition (SOA x Congruency).

\footnotetext{
${ }^{1}$ In Experiment $1 \mathrm{~b}$ only 2 blocks were administered after the warm-up blocks and participants could repeat each trial only twice.
} 
Data Analysis. Two raters classified the idiosyncratic descriptions that participants collected at the end of the Training Phase into one or more of the seven perceptual categories: Target inside Mask, Target before Mask, Dark Target, Bright Target, No Target, Rotation and Expansion. In addition, a residual category (Residual) was offered, which should be used only if the description of the participant did not fit in any of the other categories. It has been made transparent to the raters which descriptions belong to which participant. The raters were informed in detail about the literature-based categories, but they were naive regarding the two different experiments, the design and the aim of the study. The order of the participants was randomized differently for each rater. To measure the Interrater-Reliability, Cohens Kappa ( $\kappa$; Cohen, 1960) was calculated for each category and experiment separately.

Data from the Test Phase were pooled across sessions. First, we calculated the proportion of each idiosyncratic description separately for each participant, SOA and Congruency. Second, in order to report summary statistics across participants we recoded the idiosyncratic descriptions into the perceptual categories according to the judgment of both raters and calculated the proportion of each of these perceptual categories separately for each participant, SOA and Congruency. If several descriptions of one participant were rated into the same category the absolute frequencies of these descriptions were summarized for this participant. We examined the influence of SOA and Congruency on the report probability for each percept separately using a factorial design. All analyses were done by means of a Randomization test: We computed t-test statistics for the main effect of Congruency, for linear and quadratic trends of SOA and for the interaction of these trends with Congruency. We then compared the observed test statistics with a permutation distribution of test statistics given the null-hypothesis. The proportions of the permutation distribution that yield an equal or higher $\mathrm{t}$-value than the observed t-value are taken as $\mathrm{p}$-value. The permutation distribution comprised 10.000 independent simulations using single-trial data of each participant. For each simulation we randomly assigned the actual given reports of each participant to the different conditions and analyzed the simulated data set identical to the observed data set. Thus, we kept the absolute number of trials for each participant and the absolute number of each idiosyncratic description constant, only the assignment to the different SOA and Congruency conditions differed. We conducted all tests for both raters separately and reported effects as significant, only when the resampling $p$-value was significant $(p<.05)$ for both raters. 
3 | Visual perception is rich. Phenomenological evidence from metacontrast masking

\subsection{Results}

\subsubsection{Training Phase (Session 1-3)}

The total number of descriptions given at the end of the training phase was $N=90$ and $N=158$ for Experiment 1a and 1b, respectively. The exemplary illustration of the description and drawings of perceptions of one participant, as well as the time course can be found in Figure A1 of Appendix I. A collection of exemplary idiosyncratic descriptions of different participants for each perceptual category is listed in Table A1 of Appendix I. On average each participant collected $M=6.9(S D=3.8)$ idiosyncratic descriptions in Experiment 1a and $M=7.9(S D=3.1)$ descriptions in Experiment 1b. Interrater-Reliability was moderate to high for most categories in both experiments (Table 3.1). Only the categories Target inside Mask and Target before Mask showed poor to fair agreement between both rates for both experiments. Nevertheless, the data patterns are highly similar regardless on which rating the analyses are based. Therefore, we report results based only on one Rater.

\section{Table 3.1}

Interrater-Reliability of Experiment $1 a$, Experiment $1 b$ and pooled across both experiments separately for each category and Kappa averaged across all categories.

\begin{tabular}{cccc}
\hline \multirow{2}{*}{ Category } & \multicolumn{3}{c}{ Cohens Kappa ( $\boldsymbol{c})$} \\
\cline { 2 - 4 } & Experiment 1a & Experiment 1b & Experiment 1 \\
\hline Target inside Mask & 0.27 & 0.37 & 0.35 \\
Target before Mask & 0.21 & 0.58 & 0.51 \\
Dark Target & 0.76 & 0.61 & 0.67 \\
Bright Target & 0.48 & 0.65 & 0.62 \\
No Target & 0.82 & 0.72 & 0.76 \\
Rotation & 1 & 0.76 & 0.85 \\
Expansion & 0.66 & 0.96 & 0.9 \\
\hline Overall Kappa & $M=0.6$ & $M=0.66$ & $M=0.67$ \\
& $(S D=0.29)$ & $(S D=0.18)$ & $(S D=0.19)$ \\
\hline
\end{tabular}


$\mathbf{A}$

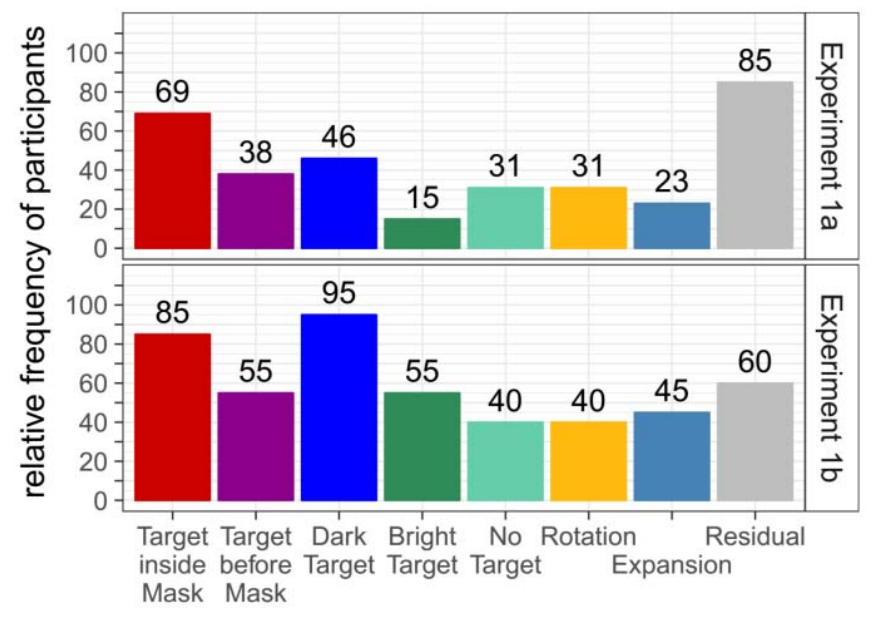

B

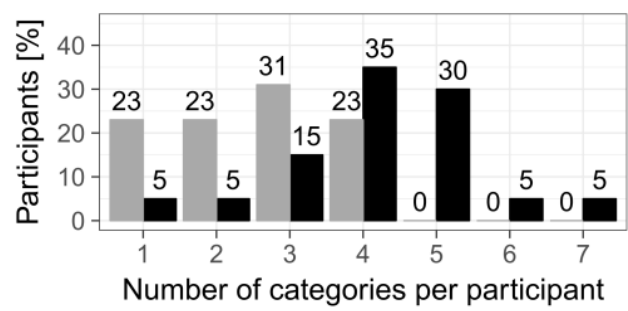

Figure 3.1. A Percentage of participants, whose description were rated inside the particular category of Experiment 1a (top) and Experiment $1 \mathrm{~b}$ (bottom). B Percentage of participants reporting different number of categories exclusive the residual category for Experiment 1a (gray bars) and Experiment $1 \mathrm{~b}$ (black bars). The absolute value of participants in Experiment 1a was $N=13$ and $N=20$ in Experiment 1b. Results based on Rating 1.

In Experiment 1a $50 \%$ of all descriptions were rated into the residual category, in Experiment $1 \mathrm{~b}$ this proportion decreased to only $20 \%$. In addition, all perceptual categories were reported by a substantial amount of participants (Figure 3.1A). The number of participants was higher in Experiment $1 \mathrm{~b}$ than in Experiment 1a for all categories, except the residual category which was used by more participants in Experiment 1a (85\% vs. 60\%). Every participant described one or more of the seven categories (Figure 3.1B). The median number of categories was higher in Experiment $1 \mathrm{~b}$ than in Experiment $1 \mathrm{a}(M=4$ vs $M=3$, $W=136.5, p=.001)$. For results based on the second rater see Figure A2 in Appendix I.

\subsubsection{Test Phase (Sessions 4-5)}

Most participants used all of their idiosyncratic description from the Training Phase in the Test Phase. Only two participants used 10 out of 13 and 14 out of 16 descriptions, respectively. Therefore, the number of descriptions used at the Test Phase was reduced for Experiment $1 \mathrm{~b}(N=153)$. In Experiment 1a in $80 \%$ of the trials of the test sessions on average $4.15(S D=1.72)$ individual idiosyncratic descriptions were used and in Experiment $1 \mathrm{~b}$ on average $4.25(S D=1.37)$. Since the data patterns from the Training Phase were very similar across both experiments we pooled the data for analysis of the Test Phase. Figure 3.2 shows the proportion of reports of each perceptual category as a function of Congruency and SOA together with the number of participants who reported the particular percept at least once. It 
3 | Visual perception is rich. Phenomenological evidence from metacontrast masking

can clearly be seen that there are marked differences in the number of reports for each percept and also differences in the time courses across SOA.

Participants reported Rotation almost only on incongruent trials with an increasing frequency of reports with increasing SOA. This effect was corroborated by a Randomization test that yielded a main effect for Congruency $(p=.0012)$, increasing trends of SOA ( $p=.0016$ and $p=.012$ for linear and quadratic trends, respectively) and interactions of Congruency with both trends $(p=.001$ and $p=.008$ for linear and quadratic, respectively). The category No Target was reported most often with intermediate SOAs, which is corroborated by a significant inverse quadratic trend of SOA $(p=.001)$. All other effects were not significant (all $p \mathrm{~s}>.25$ ). Reports of Target before Mask increased with increasing SOA especially for incongruent target-mask pairs (interaction Congruency x SOA for linear trend: $p=.079$, interaction Congruency $\mathrm{x}$ SOA for quadratic trend: $p=.019$; all other $p \mathrm{~s}>.15$ ). Reports of an Expansion were marginally more frequent on congruent than on incongruent trials $(p=.08)$ and decreased with increasing SOA $(p=.03$ for linear trend). No other effect reached significance (all $p \mathrm{~s}>.14$ ).

Reports of Target inside Mask, Dark Target and Bright Target did not yield unequivocal results: For Target inside Mask visual inspection suggested a decreasing trend of SOA, but no effect proved to be significant for both raters (main effect of Congruency $p=.03$ for Rater 1, interaction Congruency x SOA for quadratic trend: $p=.02$ for Rater 2, all other $p \mathrm{~s}>.09)$. The percept of a Dark Target was reported very often, but did not show any significant relation with Congruency or SOA for both raters (SOA for quadratic trend: $p=.03$ for Rater 2, all other $p \mathrm{~s}>.08$ ). The percept of a Bright Target was reported by only a few participants and showed the expected decreasing frequency across SOA at least according to Rater 1, but these effects did not prove significant (interaction Congruency x SOA for linear trend: $p=.007$ for Rater 1 , all other $p \mathrm{~s}>.09$, all $p \mathrm{~s}>.44$ for Rater 2). For a list of all effects based on both ratings see Appendix I Table A2. 


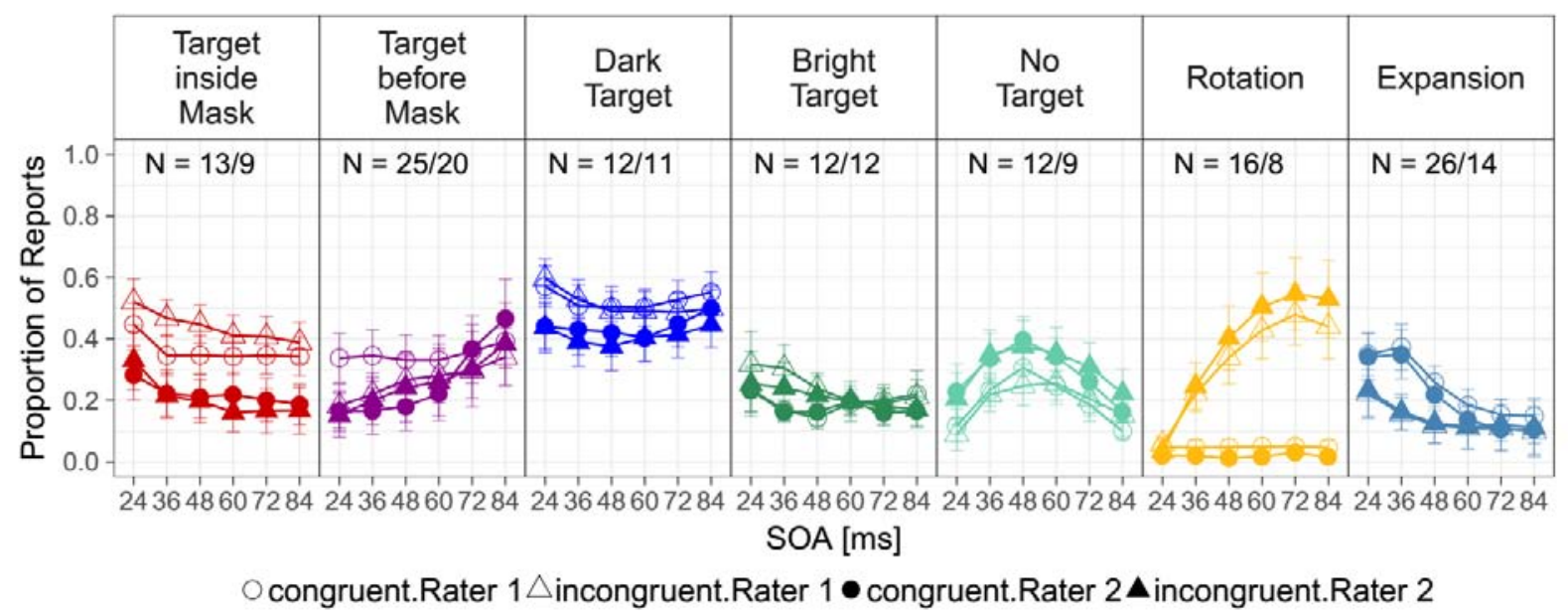

Figure 3.2. The mean relative frequencies of reports for each perceptual category as function of SOA and Congruency for Experiment 1. Error-bars depict between-subject standard errors of the mean. The digits indicate the number of participants on which each perceptual category is based, for Rater 1 and Rater 2, respectively. The maximum number of trials was $N=56$.

\subsection{Discussion}

Experiment 1 provided a first insight into the phenomenology of a metacontrast masking paradigm. The main results were straightforward: Even under conditions of reduced awareness and the usage of simple geometric figures as targets and masks, 35 of 39 participants were able to describe rich and detailed visual experiences that occurred repeatedly on different trials. These idiosyncratic descriptions were similar across participants and were related to (1) the perceived temporal order of the target-mask sequence (Target inside Mask, Target before Mask), (2) the perceived contrast of the target (Dark Target, Bright Target, No Target) and (3) motion categories (Expansion, Rotation). All seven categories were reported to a substantial amount, although the number varied widely between categories and participants. The interrater reliabilities were moderate to high except for the time related categories. These findings suggested that metacontrast results in a multidimensional experience of the target stimulus. Therefore, the use of simple unidimensional scales to measure the awareness of a target stimulus has to be questioned. Moreover, the results provided evidence that SOA and congruency affected the occurrence of the different categories differentially. This fits nicely to findings that different perceptual aspects of the metacontrast sequence follow different masking functions (e.g. Ansorge et al., 2009; Kahneman, 1967; Neumann \& Scharlau, 2007; Reeves, 1982; Weisstein \& Growney, 1969). 
3 | Visual perception is rich. Phenomenological evidence from metacontrast masking

The inverted $\mathrm{u}$-shaped function found for reports of No Target corresponds to a typical type-B-Making function widely found in metacontrast (Kahneman, 1967; Weisstein \& Growney, 1969). This finding confirmed the effectiveness of our masking procedure. Whereas discrimination tasks could only evidence the lack of information necessary to infer the shape of the target stimulus, the No-Target-Reports provided direct evidence that the stimuli used in our present and previous studies (Kahneman, 1967; Weisstein \& Growney, 1969) in fact produce strong masking without any visual experience of the target stimulus. In contrast, Dark Target and Bright Target did not show reliable variations across SOA: The report frequency of a Dark Target was on a high level at all SOAs. This may be explained by the supposition of two variations of the perception of a Dark Target, i.e. at short SOAs Dark Target may be perceived within in the mask, at longer SOAs a Dark Target may be perceived temporally before the mask. A Bright Target was reported by very few participants leading to a lack of statistical power. This finding adds to previous evidence that a brightness reversal seems to be a rather instable phenomenon that may occur only under certain conditions. For instance, Stewart et al. (2011) found brightness reversal only in a spatial forced-choice task, not in a temporal forced-choice task.

The perception of a Rotation was hypothesized to be helpful in discriminating congruent from incongruent trials at longer SOAs, a strategy supposedly leading to a type-A masking function (Albrecht \& Mattler, 2012a). Our present results show that the perception of a Rotation in fact occurs only on incongruent trials at intermediate and long SOAs. The second motion related category, Expansion, was predominantly perceived at the shortest SOA with a decreasing trend towards longer SOAs. This finding contradicts previous studies that associated an Expansion with apparent motion between target and mask (Albrecht \& Mattler, 2012a), which sometimes occurred even without concurrent experience of a target stimulus (Kahneman, 1967; Weisstein \& Growney, 1969). In contrast, in the current experiment participants described the perception of an Expansion as the impression of a target growing in size that was described, for example as a small point or circle, which started in the center of the mask and expanded outwards until it fitted into the inner contour of the mask. Thus, the percept of expanding target seems to be rather a 'filling out' as reported by Breitmeyer and Jacob (2012).

While one of the time related categories (Target before Mask) showed a decreasing trend with SOA, for Target inside Mask the expected time courses was visible, but did not yield clear statistical results. This lack of effect was possibly due to the low interrater reliability. Probably participants' descriptions did not differentiate precisely between a 
temporal separation, which is critical for the perception of a target presented before the mask and a spatial separation, which is central for the perception of a target integrated inside mask. Consequently, misclassifications of idiosyncratic descriptions could occur between the two temporal categories.

However, the time courses for the different percepts, especially the lack of effects for some percepts, have to be interpreted with caution because the statistical power is limited due to the phenomenological approach: First, some categories, for example Bright Target, were based on a small number of participants. Second, individual idiosyncratic descriptions showed a high degree of interindividual variability that was neglected by categorizing these descriptions into perceptual categories by the raters, leading to variance within each category and to a low interrater reliability. Third, the perceptual categories were far from being exhaustive as reflected by the high number of reports in the residual category. This suggests that the perceptual categories do not cover the complete visual experience. Fifth, participants' introspective or verbal skills may differ as well as their perceptual sensitivity to perceive very subtle visual differences. Accordingly, it is difficult to infer from the absence of a report whether participants in fact do not experience these percepts or if they just did not verbalized them. In spite of these limitations our approach provides ample evidence for the reliability of the different perceptual qualities within a metacontrast paradigm and constitutes direct evidence that the phenomenology of the target is not constant across conditions in a metacontrast paradigm. Moreover, the results show that qualitative aspects of perception can be captured in spontaneous reports of naive participants with moderate reliability.

\subsection{Introduction of Experiment 2}

The aim of Experiment 2 was threefold: First, in Experiment 1 most perceptual categories were reported only by a part of the participants leaving it unclear whether these individual differences are on perceptual levels or on response behavior. Therefore, Experiment 2 investigated if the individual differences in absolute report frequencies of different percepts were caused by differences in the salience of these percepts or if they were due to individual differences in perceptual abilities of the participants. Second, we sought to scrutinize the time courses, which we found in the first experiment. Third, the results of Experiment 1 suggest that visual experience in metacontrast is multidimensional and qualitatively different for different experimental conditions. Nevertheless, it remains unclear how these phenomenological differences relate to the performance in an objective 
3 | Visual perception is rich. Phenomenological evidence from metacontrast masking

discrimination task, which is more common in masking paradigms. In particular, it is of interest if specific percepts are associated with discrimination performance at specific SOAs and, moreover, if individual differences in visual experiences are associated with individual differences found in discrimination tasks (Albrecht et al., 2010; Albrecht \& Mattler, 2012a, 2016, 2016; Maksimov et al., 2011). To this end, we employed a more rigorous experimental procedure by asking for a specific percept on each trial and introduced an objective discrimination task in a further session.

\subsection{Methods}

Participants. A group of twenty-five naive students (17 female, 8 male; age from 18 to 30 years, $M=22.8$ years, $S D=3.2$ years) participated in eight sessions of 60 to 90 minutes. One participant was excluded because she could not describe the categories according to the instruction in the first session. All participants were from Georg-August University Goettingen, had normal or corrected-to-normal vision and received monetary reward. All participants were naive with regard to the aim of the study and never had participated in a metacontrast masking experiment before.

Task. In Sessions 1-7 participants had to indicate their phenomenological experience of the target-mask sequence in a yes-no task. On each trial they affirmed or negated the experience of a specific perceptual category (Target inside Mask, Target before Mask, Dark Target, Bright Target, No Target, Rotation and Expansion) by pressing one of two buttons. In Session 8 participants were asked to respond as accurately as possible and without speed stress to the shape of the square or diamond target stimuli with a button press of the left or right hand response, respectively. We instructed the participants as carefully and thoroughly as possible: They were informed about the shapes of target and mask stimuli and their spatial relationship, i.e. that the target fits neatly into the inner contours of the mask. Participants were not informed that stimulation conditions did not differ between blocks. We stressed the difficulty of the task and that there was no correct or incorrect response option. In addition, in Session 1 after a certain number of trials participants were asked to report their subjective experience as detailed as possible. For detailed instructions see Supplementary Information of Appendix II.

Stimuli, Procedure and Design. Stimuli, trial sequence and design were identical to that in Experiment 1 (Figure 2.1 in General Methods) with the following particularities: In addition to congruency of target and mask and SOA, which were varied within experimental 
blocks, we varied the perceptual category, blockwise. On each block we measured the subjective experience of one of the seven categories Target inside Mask, Target before Mask, Dark Target, Bright Target, No Target, Rotation and Expansion. The order of the categories varied pseudo-randomly across sessions for each participant so that the order was counterbalanced within participants. At the beginning of each block a prototypical description of the category was offered (see Supplementary Information of Appendix II).

Session 1 was considered as training to familiarize the participants with the task and the categories and was excluded from analysis. It consisted of one warm-up block of 8 trials and 14 blocks of 24 trials each. Each of the Congruency-SOA combinations occurred once per block. In the middle and at the end of each block participants were requested to describe the appearance of the particular category in their own words. The investigator either repeated the description of the category or corrected it if the participants seem to misunderstand the category. If participants were not able to perceive the category, they were asked to describe how they imagine the perception of the category, to ensure, that there was no misunderstanding of the definition. The experimentator stayed in the room for the whole session.

At the beginning of Sessions 2-7 participants verbally described the seven categories in their own words and performed one warm-up block of 8 trials followed by 14 experimental blocks of 52 trials each. The first four trials of each block served as adaptation trials for the new category and were excluded from analyses. The independent variables (Target x Mask $x$ SOA) of the adaptation trials were balanced across sessions for each percept. Each of the 24 combinations occurred twice in each block. Altogether 48 trials per condition (SOA $\mathrm{x}$ Congruency $x$ Category) were included in the analysis of the subjective data. In order to be able to use the F-keys comfortably, the keyboard was turned upside down. Each F-key was stuck with either a number (1-7) indicating the particular category or "no". Participants were instructed to place their index finger of the one hand on the F-key for the particular category and the index finger of the other hand on the F-key for the no-answer. The left-right assignment of the hands was balanced across participants. A note was placed in front of the participants, which indicated the assignment of the categories to the numbers. At the end of each block participants indicated the currently judged category by button press to ensure that they judged the instructed category.

Session 8 consisted of 12 blocks with 48 trials each. Each of the 24 combinations occurred twice in each block. Altogether 48 trials per condition (SOA x Congruency) were 
3 | Visual perception is rich. Phenomenological evidence from metacontrast masking

included in the analysis. The square-diamond assignment to the control keys was counterbalanced across Participants. Participants received no error feedback.

\section{Data Analysis.}

Subjective Data. To examine the effect of SOA and Congruency, we calculated a generalized linear mixed-effect regression model with a logit link function separately for each perceptual category (of the package lme4, function glmer; (Bates, Mächler, Bolker, \& Walker, 2015) using $\mathrm{R}$ Core Team (2014)). The binary yes-no response on each trial served as dependent variable. Subjects were included as random intercept, SOA and Congruency were included as by-subject random slopes to satisfy the assumption of independence (Baayen, Davidson, \& Bates, 2008). Fixed effects were Congruency (dummy-coded 2-level factor), SOA as metric linear predictor, SOA as metric quadratic predictor and the interaction between Congruency and both terms of SOA. SOA was scaled to $M=0$ and $S D=1$. We selected the best model using an automatic backward selection procedure: We started with the full model and, on each iteration, we excluded the effect with the largest $p>.05$ that was not part of any significant higher-order effect. Model selection stopped when all remaining effects had $p<.05$ or were part of a higher-order interaction (see Panis \& Schmidt, 2016). The final model was compared to the full model by a log-likelihood test.

Objective Data. Discrimination performance in Session 8 was assessed by Signal Detection Analysis in terms of discrimination sensitivity, d' (Macmillan \& Creelman, 1991). To avoid confounds with response bias we calculated d' separately for each mask and then averaged across mask (Vorberg, Mattler, Heinecke, Schmidt, \& Schwarzbach, 2004). Hit rate and false alarm rates were corrected according to the log-linear rule to avoid infinite values of $d$ ' (Hauntus, 1995). We analyzed the effect of SOA on $d$ ' by fitting linear mixed-effects models (function lmer, package lme4). SOA was entered as linear as well as quadratic fixed effects and subject as random intercept. To control for individual differences in the slope of masking functions (Albrecht et al., 2010) we entered SOA additionally as random slope. SOA variables were scaled to $M=0$ and $S D=1$. In a second step we investigated the relationship between subjective and objective data by entering the subjective data from Sessions 2-7, i.e. the relative frequencies of reported categories at each SOA, as additional predictor variable into the model. This was done separately for each category. Relative frequencies of reports were averaged across Congruency for all categories except Rotation for which we used the difference Incongruent - Congruent as predictor. 


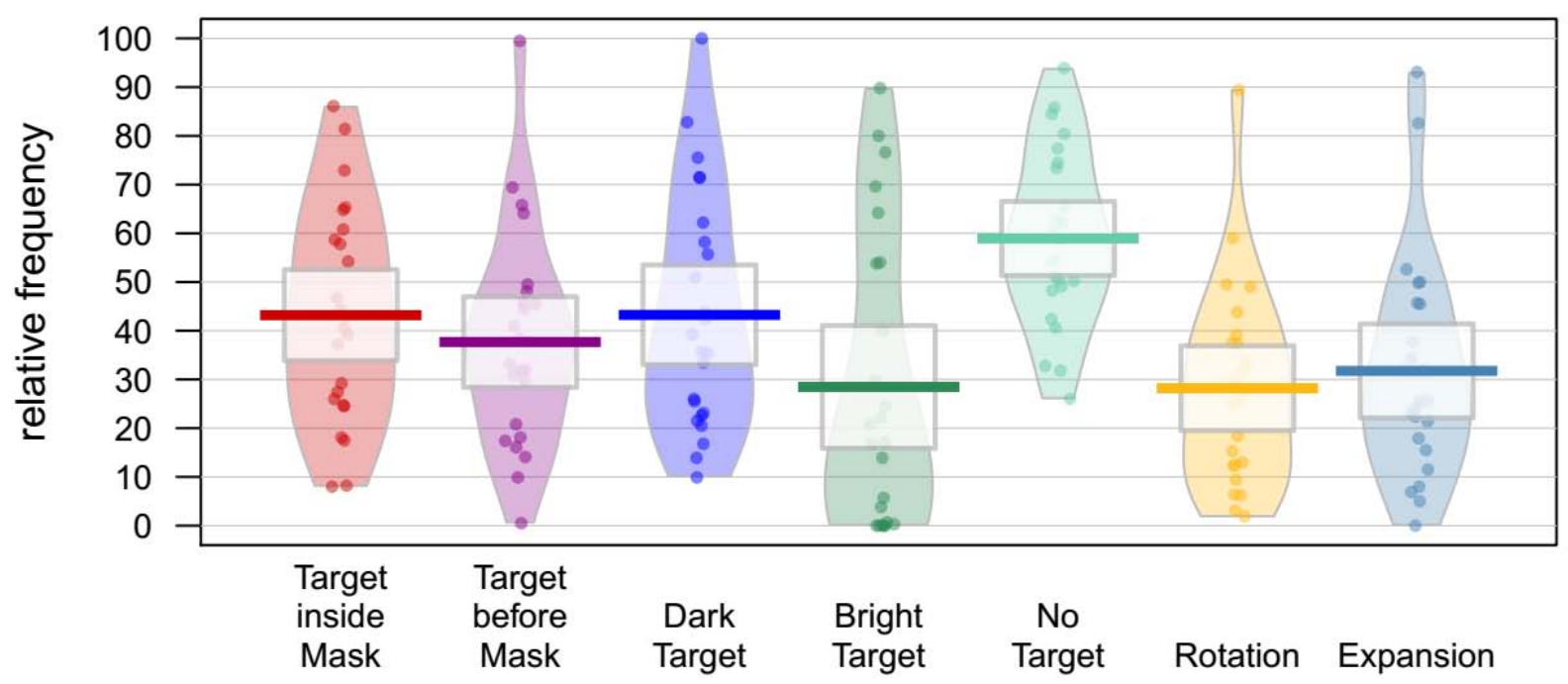

Figure 3.3. The points represent the relative frequency of reports for each participant and category as raw data. Line and Box show the mean and 95\% confidence interval for the each category. The distribution of relative frequencies for each category is expressed by the density bean. (red = Target inside Mask, violet $=$ Target before Mask, blue $=$ Dark Target , dark green $=$ Bright Target, light green $=$ No Target, yellow $=$ Rotation, light blue $=$ Expansion $)$.

\subsection{Results}

\subsubsection{Subjective Data}

Figure 3.3 depicts the number of reports for each category and participant and shows the substantial variability across categories and participants. Retest-Reliabilities for the categories were acceptable to high (Target inside Mask: $r=.77, p<.001$; Target before Mask: $r=.88, p<.001$; Dark Target: $r=.86, p<.001$; Bright Target: $r=.95, p<.001$; No Target: $r=.75, p<.001 ;$ Rotation: $r=.91, p<.001 ;$ Expansion: $r=.84, p<.001$ ).

Figure 3.4 depicts the time courses across SOA and Congruency for each category. Table 3.2 summarizes the statistical results of the final generalized linear mixed effect models of each category. The numbering in the text refers to the respective predictor in Table 3.2.

For Target inside Mask the final model included, a main effect of Congruency (1a), a negative linear trend of SOA $(1 b)$ and a positive quadratic trend of SOA (1c) indicating a curvilinear decrease of report frequencies with increasing SOA. In addition, the interaction of Congruency with the linear trend of SOA $(1 d)$ proved significant, indicating a stronger linear decrease across SOA for incongruent trials. A log-likelihood test did not reveal any difference to the full model $\left(X^{2}(1)=1.87, p=0.17\right)$. 
3 | Visual perception is rich. Phenomenological evidence from metacontrast masking

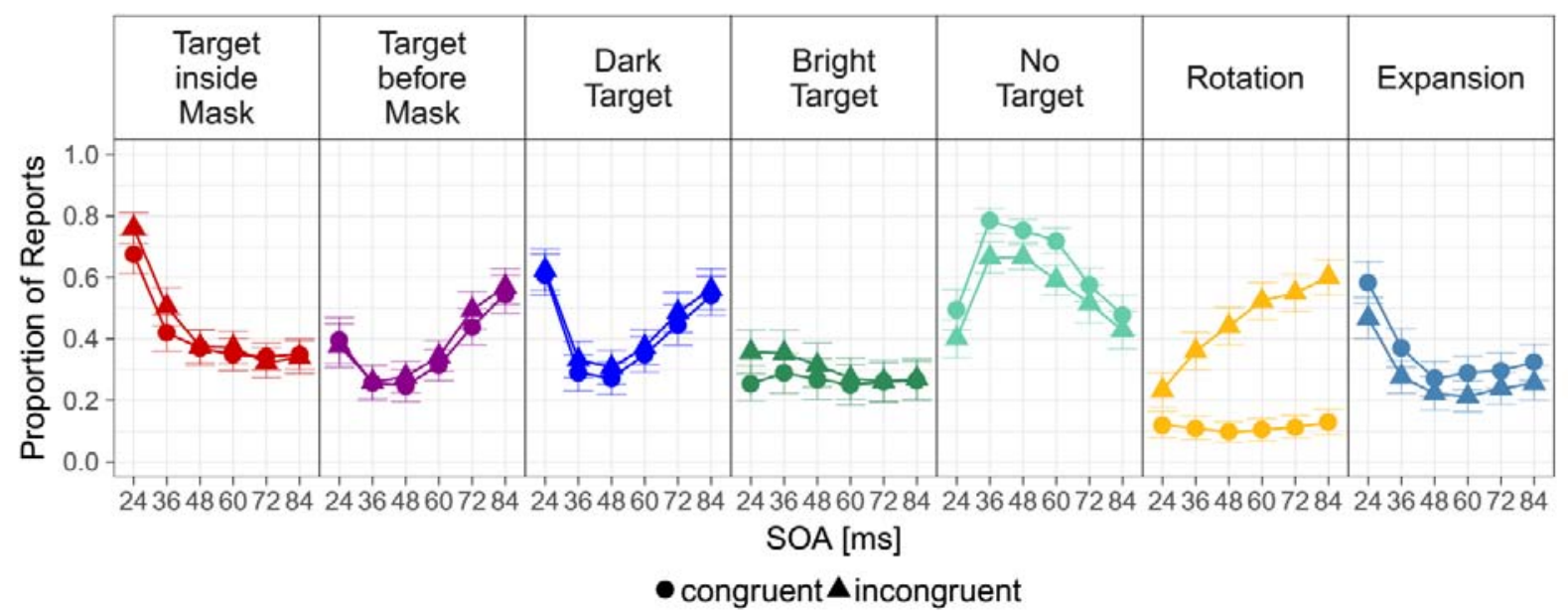

Figure 3.4. The mean relative frequencies of reports for each perceptual category as function of SOA and Congruency. Error-bars depict between-subject standard errors of the mean.

For the category Target before Mask the final model included only the linear $(2 a)$ and quadratic effects of SOA (2b). Report frequencies increased with increasing SOA in a curvilinear fashion. Note, however, that the curvilinear trend, which contradicts with the assumed increasing time course across SOA, results from higher report frequencies at the shortest SOA compare to the $36 \mathrm{~ms}$ SOA. The log-likelihood test indicated a marginally worse model fit compared to the full model $\left(X^{2}(3)=7.48, p=0.06\right)$.

For the category Dark Target the final model included a main effect for Congruency $(3 a)$ and a quadratic effect of SOA (3b). Report frequencies were higher for incongruent than congruent trials and followed a u-shaped function across SOA. A log-likelihood test did not reveal any difference to the full model $\left(X^{2}(3)=0.69, p=0.88\right)$.

The final model for the category of a Bright Target included the main effect of Congruency ( $4 a)$, the linear effect of SOA $(4 b)$ and the interaction of Congruency $\mathrm{x}$ linear effect of SOA (4c). Report frequencies of a Bright Target decreased linearly with increasing SOA especially for incongruent trials. No significant differences to the full model were obtained $\left(X^{2}(2)=0.51, p=0.77\right)$.

For the category No Target the final model was marginally worse than the full model $\left(X^{2}(1)=3.37, p=0.07\right)$. It included a main effect of Congruency $(5 a)$, a linear $(5 b)$ and quadratic effects of SOA $(5 c)$, and the interaction of the linear SOA effect with Congruency (5d). Report frequencies of No Target followed an inversed u-shaped function across SOA as indicated by a significant negative quadratic trend of SOA. In addition, No Target was reported more often on congruent trials than on incongruent trials together with a slight linear increase of reports across SOA for incongruent trials (interaction Congruency x linear SOA). 
For the category Rotation no effect was excluded from the full model. A main effect of Congruency (6a), a linear (6b) and a quadratic effect of SOA (6c) was found. Both SOA effects interacted significantly with Congruency (Congruency $\mathrm{x}$ linear SOA, $6 d$ and Congruency $\mathrm{x}$ quadratic SOA, 6e), indicating a strong curvilinear increase of report frequencies with increasing SOA for incongruent trials but not for congruent trials.

For the category Expansion the final model includes only the main effects of Congruency (7a), linear SOA $(7 b)$ and quadratic SOA (7c). The goodness of fit did not differ from the full model $\left(X^{2}(2)=3.24, p=0.20\right)$. In accordance with the assumption, a negative linear effect of SOA was found as well as a positive quadratic effect of SOA. The effect of Congruency indicates that the perception of an Expansion occurs more often on congruent trials. 


\section{Table 3.2}

Summary of the generalized linear mixed effect models, calculated for each category separately $(N=24$, Number of observations: 13824; OR = odds ratio).

\begin{tabular}{|c|c|c|c|c|c|}
\hline Target inside Mask & B & SE & $\mathbf{z}$ & OR & $p$-value \\
\hline \multicolumn{6}{|c|}{$\begin{array}{l}\text { Random effect of subject: variance }=1.79(S D=1.34) \\
\text { Random effect of SOA: variance }=0.41(S D=0.64) \\
\text { Random effect of Congruency: variance }=0.19(S D=0.43)\end{array}$} \\
\hline 1a. Congruent vs incongruent & 0.20 & 0.10 & 1.97 & 1.22 & .05 \\
\hline 1b. SOA linear & -0.54 & 0.13 & -3.99 & 0.58 & $<.001$ \\
\hline 1c. SOA quadratic & 0.48 & 0.03 & 18.50 & 1.62 & $<.001$ \\
\hline $1 d$. Congruent vs incongruent $* \mathrm{SOA}$ linear & -0.23 & 0.04 & -5.21 & 0.80 & $<.001$ \\
\hline Target before Mask & B & $\mathbf{S E}$ & $z$ & OR & $p$-value \\
\hline \multicolumn{6}{|c|}{$\begin{array}{l}\text { Random effect of subject: variance }=3.55(S D=1.88) \\
\text { Random effect of SOA: variance }=0.89(S D=0.94 \\
\text { Random effect of Congruency: variance }=0.23(S D=0.48)\end{array}$} \\
\hline 2a. SOA linear & 0.45 & 0.20 & 2.28 & 1.56 & .02 \\
\hline $2 b$. SOA quadratic & 0.48 & 0.03 & 17.86 & 1.63 & $<.001$ \\
\hline Dark Target & B & SE & $z$ & OR & $p$-value \\
\hline \multicolumn{6}{|c|}{$\begin{array}{l}\text { Random effect of subject: variance }=3.09(S D=1.76) \\
\text { Random effect of SOA: variance }=0.78(S D=0.88) \\
\text { Random effect of congruency: variance }=0.02(S D=0.15)\end{array}$} \\
\hline $3 a$. Congruent vs incongruent & 0.20 & 0.05 & 3.73 & 1.22 & $<.001$ \\
\hline 3b. SOA quadratic & 0.79 & 0.03 & 28.46 & 2.20 & $<.001$ \\
\hline Bright Target & B & SE & $z$ & OR & $p$-value \\
\hline \multicolumn{6}{|c|}{$\begin{array}{l}\text { Random effect of subject: variance }=17.06(S D=4.13) \\
\text { Random effect of SOA: variance }=1.57(S D=1.25) \\
\text { Random effect of Congruency: variance }=0.24(S D=0.49)\end{array}$} \\
\hline 4a. Congruent vs incongruent & 0.23 & 0.18 & 1.26 & 1.26 & .21 \\
\hline 4b. SOA linear & -0.74 & 0.31 & -2.41 & 0.48 & .02 \\
\hline 4c. Congruent vs incongruent $*$ SOA linear & -0.25 & 0.05 & -4.57 & 0.78 & $<.001$ \\
\hline
\end{tabular}


Table 3.2 (continued)

\begin{tabular}{|c|c|c|c|c|c|}
\hline No Target & $\mathbf{B}$ & SE & $z$ & OR & $p$-value \\
\hline \multicolumn{6}{|c|}{$\begin{array}{l}\text { Random effect of subject: variance }=1.25(S D=1.12) \\
\text { Random effect of SOA: variance }=0.70(S D=0.84) \\
\text { Random effect of Congruency: variance }=0.43(S D=0.65)\end{array}$} \\
\hline $5 a$. Congruent vs incongruent & -0.52 & 0.14 & -3.67 & 0.60 & $<.001$ \\
\hline 5b. SOA linear & -0.22 & 0.17 & -1.26 & 0.80 & .21 \\
\hline 5c. SOA quadratic & -0.63 & 0.03 & -24.90 & 0.53 & $<.001$ \\
\hline $5 d$. Congruent vs incongruent $*$ SOA linear & 0.09 & 0.04 & 2.13 & 1.10 & .03 \\
\hline Rotation & B & $\mathbf{S E}$ & $z$ & OR & $p$-value \\
\hline \multicolumn{6}{|c|}{$\begin{array}{l}\text { Random effect of subject: variance }=3.93(S D=1.98) \\
\text { Random effect of SOA: variance }=0.15(S D=0.39) \\
\text { Random effect of Congruency: variance }=0.85(S D=0.92)\end{array}$} \\
\hline $6 a$. Congruent vs incongruent & 3.27 & 0.23 & 14.39 & 26.21 & $<.001$ \\
\hline 6b. SOA linear & 0.19 & 0.10 & 1.96 & 1.21 & .05 \\
\hline 6c. SOA quadratic & 0.12 & 0.05 & 2.21 & 1.13 & .03 \\
\hline $6 d$. Congruent vs incongruent $*$ SOA linear & 0.59 & 0.06 & 9.50 & 1.80 & $<.001$ \\
\hline 6e. Congruent vs incongruent * SOA quad. & -0.37 & 0.07 & -5.57 & 0.69 & $<.001$ \\
\hline Expansion & B & SE & $z$ & OR & $p$-value \\
\hline \multicolumn{6}{|c|}{$\begin{array}{l}\text { Random effect of subject: variance }=3.33(S D=1.82) \\
\text { Random effect of SOA: variance }=0.50(S D=0.70) \\
\text { Random effect of Congruency: variance }=0.82(S D=0.90)\end{array}$} \\
\hline 7a. Congruent vs incongruent & -0.51 & 0.20 & -2.6 & 0.60 & .009 \\
\hline 7b. SOA linear & -0.49 & 0.15 & -3.34 & 0.61 & $<.001$ \\
\hline 7c. SOA quadratic & 0.47 & 0.03 & 16.62 & 1.60 & $<.001$ \\
\hline
\end{tabular}


3 | Visual perception is rich. Phenomenological evidence from metacontrast masking

\subsubsection{Objective Data}

The performance on the discrimination task is depicted in Figure 3.5. Visual inspection suggested that the slope and the absolute level of performance differed across participants: Some participants performed high at short SOAs but low at long SOAs, others showed the reverse pattern and one participant showed discrimination performance close to zero for all SOAs. On average, discrimination performance decreased with increasing SOA in a curvilinear manner. This was corroborated by a linear mixed-effects model, which showed a significant negative linear effect of SOA (beta $=-.45 ; t(23)=-4.63, p<.001$ ) and a significant positive quadratic effect of SOA (beta $=.33 ; t(23)=8.07, p<.0001$ ). For complete results see Appendix II Table B1.

Comparing the time courses of objective and subjective data reveals Target inside Mask, Bright Target and Expansion showed similar decreasing trends to $d$ '. In contrast, the reports of Target before Mask, Rotation and the inverted frequencies of No Target increased with SOAs. The double dissociation between discrimination performance and the perceptual categories suggested that the increasing information about the temporal succession of target and mask, about the spatial relation of target and mask and about the target by itself was not sufficient or was not used to identify the shape of the target. One interesting observation regarded the comparison of the discrimination performance and the inverted No Target reports at the shortest and at the longest SOA: No Target reports did not differed between both SOAs $(55.2 \%$ and $54.7 \%$ at the $24 \mathrm{~ms} \mathrm{SOA}$ and at the $84 \mathrm{~ms}$ SOA, respectively, $t(23)=.05, p=.96)$. In contrast, discrimination performance decreased from $d^{\prime}=2.03$ at the $24 \mathrm{~ms}$ SOA to $d^{\prime}=0.53$ at the $84 \mathrm{~ms} \operatorname{SOA}(t(23)=5.34, p<.0001)$. Discrimination performance decreased significantly stronger than subjective data $(t(23)=-6.31, p<.0001)$. Thus, although subjectively the same amount of information about the target was perceived at both SOAs, the content of this information was different (shape information at short SOAs, no information about the shape at the long SOAs).

To examine the correlation between subjective categories and discrimination performance, we added the report frequencies of each category as predictor variables to the LME-model of discrimination performance above. Report frequencies of Target inside Mask revealed a main effect of Category $($ beta $=.20, t(63.75)=2.69, p<.009)$ and significant interactions with both SOA variables (beta $=-.19, t(57.59)=-3.37, p=.001$ and beta $=.08$, $t(52.07)=2.32, p=.02$ for linear trend of SOA and quadratic trend of SOA, respectively) suggesting a positive correlation of Target inside Mask with $d$ ' that decreased with increasing 
SOA. Target before Mask, Dark Target and No Target showed significant main effects $($ beta $=.33, t(92.02)=4.22, p<.001 ;$ beta $=.44, t(89.27)=6.44, p<.0001 ;$ beta $=-.36$, $t(101.74)=-5.65, p<.001)$ but no significant interactions with neither linear or quadratic trend of SOA (all $p \mathrm{~s}>.10$ ). Bright Target showed a borderline significant interaction with quadratic trend of SOA (beta $=.07, t(39.55)=2.04, p=.048$, but no main effect nor an interaction with linear trend of SOA (both $t \mathrm{~s}<1, p \mathrm{~s}>.64$ ). The model including reports of Rotation revealed significant interactions with linear trend of SOA (beta $=.18, t(90)=2.79$, $p=.006)$ and quadratic trend of SOA (beta $=-.13, t(88.01)=-3.39, p=.001$ ), but no main effect $(t(89.72)=.02, p=.98)$ indicating a steeply increasing positive relation of subjective Category and $d$ '. Including reports of Expansion in the model yielded no significant main effect $(t(64.78)=.86, p=.40)$ but marginal significant interactions with linear trend of SOA $($ beta $=-.10, t(57.96)=-1.77, p=.08)$ and quadratic trend of SOA $($ beta $=.07, t(52.36)=1.96$, $p=.055$ ) indicating a possible positive correlation of Expansion with $d$ ' at short SOAs that declined with increasing SOA. For complete model statistics see Appendix II Table B2.

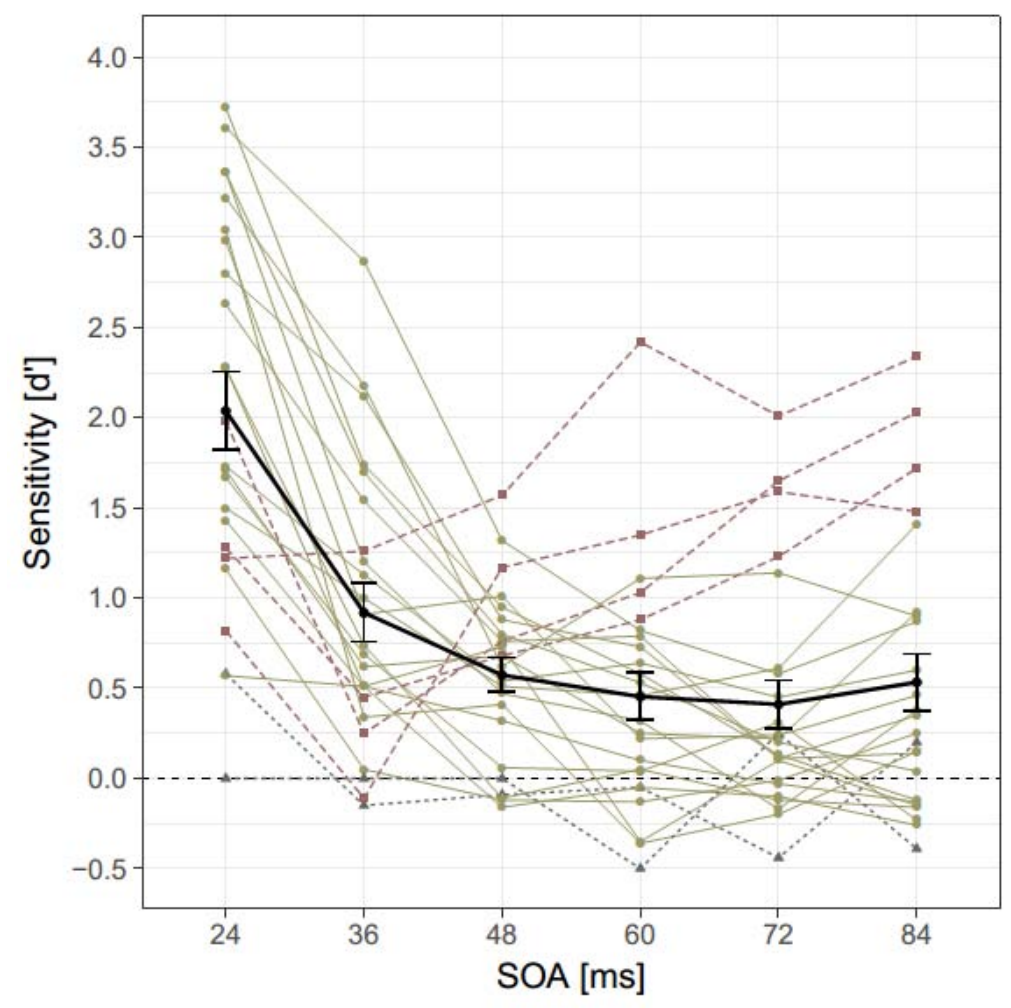

Figure 3.5. Individual masking functions with increasing time course (dashed line, light red), with decreasing time course (dashed line, green) and with constant discrimination performance hardly deviating from zero (dashed line, gray). Averaged discrimination sensitivity for each SOA (solid line, black). Error-bars depict between-subject standard errors of the mean. 
3 | Visual perception is rich. Phenomenological evidence from metacontrast masking

\subsection{Discussion}

In sum, the results of Experiment 2 were straightforward: First, the subjective data replicated the results of Experiment 1 showing distinct time courses across SOA for different categories, high interindividual variability in the report frequencies for each category and a high degree of intra-individual stability across sessions. Second, results for the objective discrimination task show typical interindividual variability in masking functions (Albrecht et al., 2010; Albrecht \& Mattler, 2012a, 2016; Maksimov et al., 2011), although all participants were highly trained in perceiving the stimuli. Third, the categories Dark Target, No Target and Rotation exhibited a double dissociation with objective performance at longer SOAs. Fourth, the categories Target inside Mask, Target before Mask, Dark Target, No Target and Rotation measured in Sessions 2-7 were related to discrimination performance. In contrast, the categories Expansion and Bright Target seemed to be not related to discrimination performance or showed only a marginal relationship with performance, respectively.

\subsubsection{Percepts related to perceived temporal order}

Both temporal related percepts showed the expected decreasing and increasing trends across SOA corroborating earlier findings on the perceived temporal order of target and mask (Neumann \& Scharlau, 2007; Reeves, 1982). Thus, with increasing SOA the frequency of an integrated percept of target and mask decreased and the frequency of two segregated events increased. In contrast to these studies, our results showed a slightly increased frequency of the segregated percept (Target before Mask) at the shortest SOA compared with intermediate SOAs. There are at least two possible explanations for these counterintuitive results: First, it may was too difficult to differentiate between segregated and integrated percepts at short SOAs. Neumann and Scharlau (2007) and (Reeves, 1982) both used SOAs up to $>=100 \mathrm{~ms}$. We used SOAs only up to $84 \mathrm{~ms}$. Thus, the phenomenological differences between maximum segregated (longest SOA) and maximum integrated (shortest SOA) were probably much smaller in our study than in the former studies. In addition, in the former studies participants were forced to choose between "integrated" or "segregated" responses on each trial, whereas we measured both percepts independently in different blocks. This may lead to a higher probability to affirm both categories on each trial participants perceive a black target in the present investigation. Second, studies on the temporal resolution of the visual system have shown that the visual system is able to differentiate two events in time that are only 10-50 ms apart (e.g. Samaha \& Postle, 2015). The exact threshold varied considerably depending on the 
stimulation conditions. Therefore, it may be that even at $24 \mathrm{~ms}$ target and mask can be perceived as successive at least on some trials and that at intermediate SOAs a masking process interferes with the perception of successive events.

The time course of perceiving a target integrated inside the Mask followed the masking function of objective performance. In line with this finding, participants who reported more often the integrated percept at short SOAs, showed better discrimination performance at short SOAs. In contrast, on average a segregated percept dissociated from objective performance at longer SOAs suggesting that although the information about the target increased with SOA, most participants were not able to use this information to identify the target shape. Nevertheless, the individual tendency to report a segregated percept was positively correlated with discrimination performance.

\subsubsection{Percepts related to target contrast}

The contrast related categories Dark Target and No Target showed pronounced ushaped or inversed $\mathrm{u}$-shaped time courses reflecting typical type-B masking functions found with luminance rating tasks (e.g. Breitmeyer et al., 2006; Neumann \& Scharlau, 2007) or subjective Rating Tasks using the PAS (Overgaard et al., 2006; Sandberg et al., 2010; Sandberg et al., 2011). This is in accordance with the assumption of maximum metacontrast suppression at intermediate SOAs (Kahneman, 1967; Weisstein \& Growney, 1969) and further validates the present approach. The finding of $80 \%$ no-target-reports at intermediate SOAs and approximately $30 \%$ at the shortest and longest SOA suggested that (1) at intermediate SOAs only sparse information about any target aspect was accessible and (2) that at longer and shorter SOAs more information was accessible but that (3) this information differed in quality: Although the amount of no-target report was equal at short and long SOAs, the discrimination performance was high at short SOAs but low at the long SOAs. Thus, the information at long SOAs was not sufficient for identifying the target shape.

Several authors have reported not only a suppressed target contrast but also a polarity reversal in target perception under metacontrast (e.g. Stewart et al., 2011; Werner, 1935). Our results contributed to, and extended these findings by showing a low but reliable proportion of Bright Target reports, which declined with increasing SOA. The fact that a Bright Target was perceived predominantly at short SOAs fits with Werner's (1935) notion that participants reported the inner contour of the mask to be brighter than the background. Stewart et al. (2011) found a brightness reversal at $20 \mathrm{~ms}$ SOA, which corresponds nicely with our results. 
3 | Visual perception is rich. Phenomenological evidence from metacontrast masking

Note, however, that there are several differences between our study and Stewart et al. (2011). First, we presented only one target and mask at fixation, whereas Stewart and colleagues presented the target left or right from fixation followed by two mask on the left and the right of fixation. Second, we asked participants directly about their visual experience, in Stewart et al. (2011), participants had to indicate on which side the target disc had been presented. In such indirect task it is not entirely clear, what criterion content participants used to detect the target (for example contrast/luminance, flicker). Therefore, we cannot say if the Bright Target reported in our study is the same phenomenon as the brightness reversal in Stewart et al. (2011). Although the Bright Target seemed to be a reliable perception we did not find any association with discrimination performance. This finding suggests that the "afterimage" we have described in earlier studies (Albrecht \& Mattler, 2012a) as cue used by some participants is not a negative afterimage.

\subsubsection{Percepts related to Motion}

The two motion related categories, Rotation and Expansion showed time courses across SOA which were in accordance with the distributions of Experiment 1. Rotation was reported mostly exclusively on incongruent trials and predominantly at long SOAs (Maksimov et al., 2011; Albrecht \& Mattler, 2012b, 2012a). Expansion showed a decreasing time course over SOA, which was not expected based on apparent motion studies, which showed a peak of apparent motion perceptions at intermediate SOAs (Hogben \& Di Lollo, 1984; Kahneman, 1967; Weisstein \& Growney, 1969). These studies described the perception of an Expansion as an impression of an objectless enlargement, which occurred even at maximum metacontrast suppression. This definition contradicted with the phenomenology described in the present study. Expansion has been described as an enlargement of the target within in the mask. In Experiment 2, 21 participants sketched an Expansion as a movement in the center of the mask and eight of them also described to perceive a target which grows in the center of the mask. In contrast, only three participants perceived a rotation as interplay between target and mask with both stimuli being visible, whereas 18 participants described a rotation without experiencing a target. Consequently, differences in time course as well as differences in the phenomenology of both motion percepts made it reasonable that they cannot be explained with the same apparent motion mechanism. The phenomenological description of a target which grows in size, has phenomenological similarities with filling-out processes of surface contrast (Breitmeyer \& Jacob, 2012). Breitmeyer and Jacob (2012) traced filling- 
out processes in metacontrast masking paradigms with differences in the temporal dynamic between surface and contour completion, which caused a perception of a stronger degraded contrast of the edges compare to the center of the target (Petry, 1978; Werner, 1935). Breitmeyer and Jacob (2012), showed that with increasing SOA the surface completion was progressing, until at long SOAs the target was perceived entirety. Breitmeyer (2014) stated that the perceptual filling-in is not completed instantaneous, but takes a short time interval. Contrary to the results of Breitmeyer and Jacob (2012), in our investigation the surface completion seemed to be completed within one single SOA, but seemed to be slowly enough to be perceived by the participants as a successive completion.

\subsection{General Discussion}

The present study provided a systematic measurement of the phenomenology in a metacontrast paradigm. The results showed that naive participants described rich and detailed visual experiences comprising temporal aspects of target and mask, contrast related aspects and motion related aspects that showed meaningful time courses across SOA and, which have been associates with metacontrast in earlier literature. These findings validated our introspective approach and provided evidence that participants were able to reliably describe their own experiences.

This speaks in favor for the idea that each category represented a unique perception of a different aspect of the target, whose appearance did not just reflect a trial-by-trial fluctuation in the perception, but depended on the experimental conditions. Regarding the simplicity of the spatial layout of the low-level stimuli used in the present study, it is surprising to find and validate seven perceptual categories. In addition, the composition of the different aspects of the target described a detailed and rich phenomenology of the target. This supports the assumption that the experience of the target varies not only quantitatively but qualitatively across conditions in a metacontrast masking paradigm (Jannati \& Di Lollo, 2012; Sackur, 2013).

The fine-tuned variations of the categories across SOA are a persuasive argument against the assumption that the subjective impression of a detailed representation of the world only bases on a perceptual illusion (Kouider et al., 2010). Instead, the perception of subtle difference in a difficult perceptual task, which varied gradual with the parametric manipulation, speaks in favor for trustworthiness of subjective reports. We offered an approach which allows capturing the phenomenology in an experimental setting, without 
3 | Visual perception is rich. Phenomenological evidence from metacontrast masking

tapping into the trap the first introspective approaches in the beginning of the twentieth century did, where a lot of variability in the results have been found between laboratories (Velmans, 2007) and most of the hypotheses could not be confirmed (Vermersch, 1999). Therefore, our approach allows a rehabilitation of phenomenological measures in cognitive psychology, which has been cast away as unreliable data (Nisbett \& Wilson, 1977).

\subsubsection{Multidimensionality of target appearance}

The dissociation between the poor discrimination performance at long SOAs and the concurrently rich subjective experiences of the target at this range of SOAs highlights one core finding of the present study: Every task in a masking paradigm probes only a restricted amount of information about the target. There is a correspondence between the objective measure and some aspects of the subjective experience, but the awareness of the stimulus would be severely underestimated if only objective performance would be used to capture conscious perception. This investigation highlights the importance to consider the whole phenomenology instead of high-level categories, to capture the visual experience exhaustively (Reingold \& Merikle, 1988). Altogether, this challenges the usage of one-dimensional subjective or objective scales, like global visibility ratings or discrimination performance, which are unable to take into account variations in the criterion content.

\subsubsection{Individual Differences}

Despite identical stimulation conditions participants showed stable and qualitative differences in the discrimination performance under metacontrast: For some participants, performance decreased with increasing SOA, for others performance increased. This phenomenon has been replicated multiple times in our own lab (Albrecht \& Mattler, 2012b, 2012a, 2016; Fleischhauer, Miller, Enge, \& Albrecht, 2014) as well as by others (Maksimov et al., 2011). We had linked these differences to differences in the visual experience of the target mask sequence (Albrecht \& Mattler, 2012a) and to differences in the weighting of underlying processes (Albrecht \& Mattler, 2016). In particular we proposed one process that leads to the perception of an "afterimage", describing the perception of a Target inside Mask at short SOAs and one process that leads to the perception of apparent (rotational) motion at long SOAs (Albrecht et al., 2010; Albrecht \& Mattler, 2012a, 2016). The current results added more evidence to the link between subjective perception and objective performance. First, participants differed widely in their reported visual experiences in both experiments. 
Thus, these differences could not be attributed to differences in verbal abilities. Second, at short SOAs individual discrimination performance was higher for participants that reported more often a Target inside Mask, but it was not related to the perception of an Expansion and only marginal related to the perception of a Bright Target. Thus, we conclude that the "afterimage" mentioned in our earlier studies (e.g. Albrecht \& Mattler, 2012a) is probably caused by mechanisms of visual persistence rather than a negative afterimage. Third, at long SOAs individual discrimination performance was higher for participants that reported more often a Rotation. However, although almost all participants reported a Rotation at long SOAs, average discrimination performance was low at long SOAs for most participants. Thus, although they perceived a Rotation they did not use this cue to discriminate the target. These findings corroborate our earlier results that participants did not only differ in the ability to perceive specific perceptual cues, but they also differed in the degree to which they exploited this cue (Albrecht \& Mattler, 2012a). Fourth, the perception of a Dark Target, a Target before Mask contributed to the discrimination performance, but independent of the SOA. Fifth, the visual experience of a Bright Target or of an Expansion probably did not contain information about the shape of the target. They may reflect processes that underlie metacontrast masking but that do not affect the processing of the target shape. Note, however, that the relation between objective performance and the perception of an Expansion was marginal significant, therefore, more research is necessary to draw final conclusions. Thus, we have identified several cues that are differently perceived and used by participants. These percepts are candidates for reflecting multiple underlying processes that together constitute an individual masking function.

\subsubsection{Perceptual Learning}

Perceptual learning refers to the improvement in the performance on a perceptual task by practice (Ahissar \& Hochstein, 2004). It is widely known that perceptual learning affects the performance in metacontrast masking (Hogben \& Di Lollo, 1984; Schwiedrzik et al., 2011; Ventura, 1980). In Ventura (1980) and Hogben and Di Lollo (1984) practice lead to a reduced of the u-shaped masking function, because of an increase in performance across the whole range of SOAs. The authors explained the effect by a change of criterion content, i.e. participants changed the perceptual cue, on which they based their judgment and gradually learned to utilize it over the course of the experiment. In contrast, Albrecht et al. (2010) showed that individual masking functions got more and more pronounced as practice 
increased. They explained their findings in the framework of reversed hierarchy theory (Ahissar \& Hochstein, 2004): In a first learning phase a reliable perceptual cue is identified, in a second phase perceptual learning leads to a more and more efficient utilization of this specific cue. Depending on the exact nature of the cue, participants developed either an increasing masking functions or a decreasing/u-shaped masking function. Schwiedrzik, Singer, and Melloni (2009) found that practice in discriminating the shape of targets in metacontrast masking not only improved the discrimination performance but also improved subjective awareness ratings on a perceptual awareness scale. Consequently, subjective awareness was influenced by training in an objective task.

In the present study participant practiced the subjective awareness of different perceptual cues within the target mask sequence and performed a discrimination task afterwards. Does this extensive practice in subjective awareness (over 7 sessions) affect performance in an objective task? Since we have only post-training data on performance, we cannot draw final conclusions. However, we do not see any sign for substantial perceptual learning effects: The average masking function is clearly type-B and most participants showed low discrimination performance at long SOAs. Nevertheless, almost all participants reported a rich visual experience of the target at long SOAs (Dark Target, Target before Mask, Rotation). Thus, although they were aware of perceptual cues that could help to discriminate the target at long SOAs, participants could not or did not utilize them. One possible explanation is that the subjective reported percepts did not comprise information about the shape so that learning to see a specific percept could not lead to improved shape discrimination. On the other hand, our data show that participants who were more prone to see a dark target temporally segregated from the mask were better in the discrimination task. In addition, at least the rotation cue can in principle be utilized in the discrimination task. In this regard, our result confirm and extend earlier findings of a dissociation between the ability to see a certain perceptual cue and the ability to utilize this cue (Albrecht \& Mattler, 2012a). Future research is necessary, to what extent learning takes place only for individually preferred perceptual cues (see Albrecht \& Mattler, 2012a).

\subsubsection{Limitations}

Two limitations of the current study have to be mentioned: First, the restricted SOA range between $24 \mathrm{~ms}$ and $84 \mathrm{~ms}$ may have resulted in low variability in visual experience and therefore may have increased the difficulty of the task. For instance, we would expect a more 
pronounced, increasing time course of Target before Mask if we had employed additional longer SOAs. In a similar way other percepts may be influenced by the context of presented stimuli. However, despite the restricted SOA range we found reliable effects of SOA in all categories, validating our conclusions despite this limitation.

Second, we treated the different percept strictly independent of one another. But we do not claim that percepts are in fact independent of one another. Most probably they are not. The fact that some of the idiosyncratic descriptions given in Experiment 1 contained more than one perceptual category may be interpreted as a sign for dependency but based on the present data we cannot draw conclusions. Future research should investigate the dependencies between percepts to reach a more detailed picture of the phenomenology. In addition, this would give the possibility to link phenomenology to underlying processes.

\subsubsection{Conclusion}

This investigation was the first approach to systematically cover the phenomenology in a metacontrast masking paradigm. The results of all experiments provide evidence for the reliability of the different perceptions extracted from literature. First, all categories were perceived by a substantial amount of participants. Second, each category showed a unique temporal relationship with SOA, which were mostly in accordance with the time courses described in metacontrast literature. This speaks in favor for the idea that each category represented a unique perception of a different aspect of the target, whose appearance did not just reflect a trial-by-trial fluctuation in the perception, but depended on the experimental conditions. Third, the divergence between the partly poor performance at long SOAs and the concurrently rich and comprehensive subjective experiences of the target at this range of SOA highlights the problem of measuring conscious visual perception with a one-dimensional forced-choice task only. There is a correspondence between the objective measure and the subjective experience, but the awareness of the stimulus would be severely underestimated, if only performance would be used to measure conscious perception.

Regarding the simplicity of the spatial layout of the low-level stimuli used in the present study, it is surprising to find and validate seven perceptual categories. The perception of subtle difference in a difficult perceptual task, which varied gradual with the parametric manipulation, speaks in favor for trustworthiness of subjective reports. In addition, the composition of the different aspects of the target described a detailed and rich phenomenology of the target. This supports the assumption that the experience of the target varies not only 
3 | Visual perception is rich. Phenomenological evidence from metacontrast masking

quantitatively but qualitatively across conditions in a metacontrast masking paradigm (Jannati \& Di Lollo, 2012; Sackur, 2013). Altogether, this phenomenological investigation challenges the usage of one-dimensional subjective or objective scales, which are unable to take into account variations in the criterion content and highlights the importance of fine-tuned perceptual categories, to capture the visual experience exhaustively. 
4 | Phenomenological evidence for multiple processes underlying metacontrast masking

\section{Phenomenological evidence for multiple processes underlying metacontrast masking}

\subsection{Abstract}

Variations in the stimulus onset asynchrony (SOA) were found to influence the perception of the target in several aspects in metacontrast masking paradigms. Different perceptions, showing the same time course across SOA, were used to investigate whether there is evidence for independent processes underlying metacontrast masking. Two perceptions were found to occur mostly at short SOAs, the perception of an expanding target and the perception of a target integrated inside the mask. Another pair of perceptions depicts an increasing time course, the perception of a rotation, resulting from form incongruent targetmask pairs and the perception of a target temporally separated from the mask. For each pair of perceptions it was examined whether they occur together on a trial or independent of each other. Evidence for three different process underlying metacontrast masking was found. The perceptions at short SOAs depicted a dependency, which was interpreted as evidence for one process eliciting both perceptions. The perceptions at long SOAs seemed to be independent of each other, which provided evidence for two processes both associated with one of the perceptions. These results can be interpreted as evidence for the multidimensionality assumption of metacontrast masking. Thereby, the SOA seemed to determine the occurrence of the processes, whereas the Congruency rather influenced which perception is more likely to be elicited by the underlying process. 


\subsection{Introduction}

Metacontrast is a widely explored phenomenon, but it is still an open debate how to explain the impairment of the perception of the target by a following mask. There is evidence that metacontrast masking is a multidimensional phenomenon, with differences in the perceptual appearance of the target at short and long SOAs (Sackur, 2013). Metacontrast masking is a special kind of backward masking, where a target stimulus is followed after a variable stimulus-onset-asynchrony (SOA) by a non-overlapping masking stimulus with adjacent contours (for review see Breitmeyer \& Öğmen, 2006). To observe the influence of the mask on the preceding target stimulus, the visibility of the target as a function of SOA can be measured. As a peculiarity of metacontrast masking, in comparison to other types of masking often non-monotonic, u-shaped masking functions can be obtained (for review see Breitmeyer \& Öğmen, 2006).

Two independent, monotonic processes were assume to cause the u-shaped masking function (Neumann \& Scharlau, 2007; Reeves, 1982). At short SOAs, an integration process was assumed that was supposed to cause the perception of a simultaneously presented target and mask, with the target integrated within the mask. At long SOAs a segregation process was assumed, which led to the perception of a temporal segregation between target and mask. Kahneman (1968) stated that the criterion content, the perceptual cue participants used to judge about the target varies with SOA. Jannati and Di Lollo (2012) showed that at short SOAs integration diminished the perceptual cue participants used to perform the tasks, whereas at long SOAs the target segregated from the mask was easily to perceive. In accordance with the consideration of metacontrast as a multidimensional phenomenon, there is evidence for independent processes underlying the ascending and descending branch of the metacontrast function. Ishikawa, Shimegi, and Sato (2006) showed that the sensitivity for differences in contrast, orientation or spatial frequency varied between short and long SOAs in a metacontrast masking paradigm. At short SOAs Ishikawa et al. (2006) assumed a fastconducting, less orientation-tuned and less contrast-sensitive pathway, broadly tuned for spatial-frequencies, with a higher sensitivity for lower spatial-frequencies. At long SOAs they expected a slow-conducting and orientation-specific and contrast-sensitive pathway, sharply tuned for spatial-frequencies, with a higher sensitivity for higher spatial-frequencies. All together there is evidence for independent processes underling the braches of the metacontrast function, which are accompanied by differences in the perception of the target. 
4 | Phenomenological evidence for multiple processes underlying metacontrast masking

\subsubsection{Evidence for three underlying processes}

Albrecht and Mattler (2016) offered a new approach to investigate different processes underlying metacontrast. They manipulated the appearance of the stimuli in a way that different perceptual cues were diminished which helped to identify the target at certain SOAs. The authors used a so-called standard metacontrast paradigm with black stimuli on white background. Targets and masks were diamond- and square-shaped, resulting in form congruent as well as incongruent target and mask combinations (see Figure 2.1A). The masks had a star-shaped inner contour, with both target shapes fitting neatly inside.

Based on discrimination performance two latent variables were uncovered with a factor analytic technic. One variable showed increasing factor loadings with increasing SOAs and was therefore called Type-A factor. The other variable had higher loadings at shorter SOAs and was named Type-B factor. According to previous results, Type-A factor was associated with the usage of a rotation to discriminate the target (Albrecht \& Mattler, 2012b, 2012a). A rotation was assumed to be a special kind of apparent motion which results from the interplay of incongruent target and mask combinations. Based on the mask shape, the rotation can be used to draw conclusions about the shape of the target. A rotation perception indicates that the target shape is opposite to that of the mask, whereas both stimuli have same form if no rotation is perceived.

The Type-B factor was associated with a target discrimination based on a black or white afterimage, visible in white star of the mask (Albrecht \& Mattler, 2012b, 2012a, 2016). Albrecht and Mattler (2016) hindered each of the two perceptual cues by modifying the standard metacontrast paradigm in two different ways. First, to prevent the perception of a rotation only neutral, which means star-shaped masks, were used. Accordant to the hypotheses, evidence was found that this manipulation selectively hindered process-A, which was associated with the perception of a rotation. Second, they used single-transient secondorder targets and masks, which consisted of random noise patterns. The shapes of both stimuli were created by locally changing the transient signal within the uniform random noise pattern by another uniform random noise pattern, which caused strong transient illusory contours appearing for a brief moment. This manipulation prevented the perception of an afterimage and was found to selectively hindered process-B. A factor analysis based on the discrimination performance of the modified stimuli yielded evidence for three underlying factors: At short SOAs, type-B factor was identified and at long SOAs, Type-A as well as an 
additional Type-C factor. The latter was preserved by the elimination of a rotation percept. They associated this factor with a form independent, more general visibility at long SOAs.

\subsubsection{Phenomenological investigations to examine underlying processes}

Since Albrecht and Mattler (2016) provided evidence that each process was associated with a unique perception, the consideration of the phenomenology in a metacontrast masking paradigm should be a promising approach to analyze underlying processes. In Experiment 1-2 of this thesis the phenomenology in the standard metacontrast masking paradigm of Albrecht and Mattler (2016) was investigated. In Experiment 1 participants were asked to report spontaneously on each trial their individual perception of target and mask without being influenced at all. For seven perceptual categories unique relationships with SOA and Congruency were found, which were in accordance with the time course reported in the metacontrast literature. The time courses have also been replicated by the second experiment of this thesis, where the seven categories were introduced to the participants. These results provided striking evidence for the assumption that the perception of the target varied qualitatively in dependence of spatial and temporal relations between target and mask. In addition, some of the categories showed similar time courses. First, two perceptions showed a decreasing time course with SOA, the perception of a target integrated inside the white star of the mask (Target inside Mask) and the perception of a target, which expanse in size (Expansion). Second, the perception of a temporal segregation between target and mask (Target before Mask) and the perception of a rotational movement (Rotation) both increased with SOA. Whereas the category Target before Mask did not show a difference in the time course between congruent and incongruent trials, the perception of a Rotation increased only at incongruent trials and stayed at a constant low-level at congruent trials.

\subsubsection{Processes underlying short SOAs}

Based on the results of Albrecht and Mattler (2016) three independent processes can be assumed. At short SOAs, they found evidence for only one process associated with the perception of an afterimage visible in the center of the mask. In contrast, there is evidence for two independent processes underlying short SOAs. Stoper and Mansfield (1978) showed that masking function of the contour contrast ratings differed from the masking function of the surface contrast ratings which was replicated by an computational simulation (Arrington, 1994). Evidence for independent surface and boundary processes were found at short SOAs in 
4 | Phenomenological evidence for multiple processes underlying metacontrast masking

metacontrast paradigms (Bachmann, 2009b; Breitmeyer et al., 2006). The comparison of surface brightness matching task and target contour judgment task yielded that the SOA for optimal suppressing the target's contour was 10-20 ms and preceded the SOA with maximal surface suppression by $30 \mathrm{~ms}$ (Breitmeyer et al., 2006). The authors interpreted the differences in time course as evidence for separate cortical mechanisms for the processing of object contour, respectively surface features. These results have been confirmed by Bachmann (2009b), who concluded that metacontrast involves different processes with their own characteristic time course, responsible for surface and contour processing.

A commonality of both studies was to capture the perception of different stimulus features to find evidence for multiple processes underlying short SOAs. In contrast, Albrecht and Mattler manipulated the stimuli without explicitly capturing differences in the target experiences. This suggests that the consideration of different target features may be necessary to find evidence for different underlying processes. In accordance, the phenomenological investigation of metacontrast (Experiment 1-2) showed that at short SOAs different aspects of the target can be perceived, the perception of an expanding target and a target integrated inside the mask. It is therefore an open question whether the consideration of these two perceptions, both showing a decreasing time course across SOA, provide evidence for one or two underlying processes.

\subsubsection{Processes underlying long SOAs}

At long SOAs Albrecht and Mattler (2016) found evidence for two independent processes, process-A and process- $\mathrm{C}$, relating to a rotational apparent motion and the visibility of the segregated target, respectively. Apparent motion was described as the illusory perception of a smooth movement between two static objects or flashes of light, which were presented one after the other, in optimal relation of spatial and temporal distance (Wertheimer, 1912). In contrast to the results of Albrecht and Mattler (2016), several authors concluded that apparent motion and metacontrast share the same underlying mechanisms (e.g. Kahneman, 1967; Schiller \& Smith, 1966). The assumption was based on the u-shaped function found for both, apparent motion (Breitmeyer, Battaglia, \& Weber, 1976; Breitmeyer, Love, \& Wepman, 1974) and metacontrast displays (Breitmeyer et al., 1974). For both phenomenon also similar practice effects were found, with a constant improvement across sessions (Hogben \& Di Lollo, 1984). Kahneman (1967) even defined metacontrast as a special kind of apparent motion, were the target is moving in an impossible motion to both 
sides of a flanking mask. He concluded that the visual system suppressed this physically impossible motion. Inverted u-shaped functions across SOA were found with maximum metacontrast, respectively apparent motion at intermediate SOAs (Kahneman, 1967), which has also been reported by previous studies (Fehrer \& Biederman, 1962; Fehrer \& Raab, 1962; Schiller \& Smith, 1966; Toch, 1956).

With adopted settings Weisstein and Growney (1969) were able to replicate the results of Kahneman (1967), that both phenomenon yielded a u-shaped function with optimal apparent motion occurring under maximum metacontrast. However, they also showed that the maximum as well as the shape of the metacontrast function was much more affected by variations of the visual angle between target and mask than the apparent motion function. In addition, metacontrast was much more sensitive to changes in viewing conditions (monoptic, dichoptic) and the energy ratio between target and mask (luminance, duration) than apparent motion. Since both phenomenon did not show the same behavior across conditions, Weisstein and Growney (1969) concluded they were not one and the same phenomenon. Thereby, the authors rejected the hypotheses that metacontrast is just a special case of apparent motion. Stoper and Banffy (1977) found evidence for an inverted u-shaped apparent motion function, which showed the same time course as the metacontrast function itself. In addition, with neighboring stimuli flanking the masks on both sides, metacontrast as well as apparent motion were diminished (Stoper \& Banffy, 1977, Experiment 1). Even if these results may speak in favor for the similarity of both phenomenon, Stoper and Banffy (1977) also found a dissociation between apparent motion and metacontrast under conditions of eccentric fixation or the increase of the distance between target and mask (Experiment 2). Both manipulations eliminated apparent motion, but left metacontrast mostly untouched. Based on these results the authors assumed that the so far unknown mechanisms of apparent motion does not seem to be necessary for metacontrast, thereby rejecting Kahneman's (1967) apparent motion theory of metacontrast that apparent motion is necessary for metacontrast.

The rotation percept was found to be less diminished by the metacontrast, than the perception of the target shape itself (Ansorge et al., 2007; Ansorge et al., 2009). Ansorge et al. (2007) concluded that the perception of a rotation and the shape of the target based on different stimulus information, which supports the assumption that there seems to be a difference in processing, whether the target is perceived as such or an apparent motion arising out of interplay between target and mask. To sum up, even if previous results spoke in favor for the similarity of apparent motion perception and target visibility at metacontrast masking paradigms (Kahneman, 1967; Schiller \& Smith, 1966), there is counterevidence for the 
4 | Phenomenological evidence for multiple processes underlying metacontrast masking

independence of both phenomena (Albrecht \& Mattler, 2016; Ansorge et al., 2007; Ansorge et al., 2009; Stoper \& Banffy, 1977; Weisstein \& Growney, 1969).

\subsubsection{Rational of Experiment 3}

To approach the question whether the phenomenology in a metacontrast masking paradigm supports the assumptions of three underlying processes, on each trial two categories were captured either the combination of Target inside Mask and Expansion or the combination of Target before Mask and Rotation. By capturing two responses on each trial it can be examined if the perceptions emerge mutual or exclusive of each other. Evidence for one underlying process eliciting both perceptions would be provided, if both perceptions either occur together on a trial or both do not occur. Whereas perceptions elicited by two different processes, should occur independently of each other.

Based on the results of Albrecht and Mattler (2016) at short SOAs, one process is assumed, therefore, a dependency between the perception of an expanding target and a target integrated inside the mask should be found. Consequently, in trials were an Expansion is perceived, it should be more likely to perceive a Target inside Mask and vice versa. At long SOAs, based on the results of Albrecht and Mattler (2016), an independency between the perception of a rotational movement and a target segregated from the mask should be found. In contrast, Kahneman (1967) original stated that metacontrast is just a special case of apparent motion. If this were the case, the perception of a rotational apparent motion should depend on the perception of the target itself. Because apparent motion usually occurs under condition of maximum metacontrast masking (Fehrer \& Biederman, 1962; Fehrer \& Raab, 1962; Kahneman, 1967; Schiller \& Smith, 1966; Toch, 1956), in trials where apparent motion is perceived the target should be less perceived and vice versa.

Previous studies only investigated the effects of the SOA on the formation of underlying processes (Albrecht \& Mattler, 2016; Neumann \& Scharlau, 2007; Reeves, 1982). But the influence of the spatial parameter, more precisely the Congruency, is unknown so far. For the perception of a rotational movement a strong influence of the Congruency has been found, whereas the other perceptions only showed a slight effect of Congruency (Experiment 1-2). Therefore, it remains an open question whether the spatial parameter influences the underlying processes in a similar way than the temporal parameter. 


\subsection{Methods}

Participants. Twenty-four ( 5 male, 19 female) naive students of the University of Goettingen between 18 and 32 years $(M=22.5$ years, $S D=3.1$ years $)$ participated in 4 sessions. The first session lasted about 90 minutes, the other sessions 60 minutes. One participant was replaced, because his sessions lasted one hour longer than planned, indicating that the task was disproportionate difficult for him, not representing the average of our sample. All participants had normal or corrected to normal vision and received monetary reward. All gave their informed consent.

Task. In each session participants were asked to give two yes-no answers on each trial by pressing the particular button, one answer for each of the two perceptual categories. It varied blockwise whether the perceptions of a target integrated inside the mask (Target inside Mask) and an expanding target (Expansion) were recorded on each trial or the experience of a target temporally segregated from the mask (Target before Mask) and a rotational movement (Rotation). The definition of the four categories based on the results of Experiment 1-2. For a detailed instruction of the perceptual categories see supplementary information of Appendix III. Participants were informed that they do not need to perceive the form of the target to affirm the categories and that the target may not be perceived as a dark black, but as a light gray figure.

Stimuli, Procedure and Design. Stimuli and trial sequence were identical to the description in General Methods (Figure 2.1). On each trial two perceptual categories were captured, either the combination of Target inside Mask and Expansion or the combination of Target before Mask and Rotation. Participants were allowed to respond at the earliest $600 \mathrm{~ms}$ after the presentation of the mask. The measurement of the two pairs of categories varied block-by-block. It was counterbalanced across participants and sessions with which combination the session started. For a detailed procedure see supplementary information of Appendix III.

The first session was a training session, which was excluded from the analysis. The experimenter stayed in the laboratory for the whole session. The aim of this session was to familiarize the participants with the task and the definition of the categories. Session 1 started with a slow warm-up block with 16 trials with each of the possible target and mask combination four times. At the first 8 warm-up trials participants were requested to press the assigned buttons of one pair of categories and at the other 8 trials the buttons of the respective other pair of categories. This was done to familiarize the participants with the array of the 
4 | Phenomenological evidence for multiple processes underlying metacontrast masking

keyboard and the general procedure of the experiment. Eight blocks followed with 24 trials each. Independent variables Target (square vs. diamond), Mask (square vs. diamond) and SOA $(24,36,48,60,72,84 \mathrm{~ms})$ varied pseudo-randomly within each block so that each of the 24 combinations were repeated one time in each block. In Block 2-5 after 12 trials participants were requested to report their experience of the particular categories. (for example "Describe your perception of trials on which you affirm a Rotation". Or "Describe your perception of trials on which you affirm a Target before Mask"). The experimenter corrected the participant in a standardized way or repeated the definition of the categories. At the end of Block 6-9, i.e. after 24 trails, participants were requested to report their perception of each of the four possible combinations (for example "Describe your perception of trials on which you affirm a Rotation and a Target before Mask". Or "Describe your perception of trials on which you affirm a Rotation but negate a Target before Mask" and so on).

Session 2-4 consisted of 14 blocks with 48 trials each. Independent variables, Target (square vs. diamond), Mask (square vs. diamond) and SOA (24, 36, 48, 60, 72, $84 \mathrm{~ms}$ ) varied pseudo-randomly within each block so that each of the 24 combinations were repeated two times in each block. Altogether in Session 2 until 4, 2.016 trials have been presented with 84 trials per condition (SOA x Congruency $\mathrm{x}$ Categories). After each session participants described and sketched their experience of each of the four categories.

Data Analysis. To investigate the in-, dependency of the categories, four generalized linear mixed-effect regression models were calculated with logit link function using the glmer function of the lme4 package (Bates et al., 2015) of the R Core Team (2014). For each category one model was calculated. The binary yes-no responses of the particular category on each trial served as criterion variable, whereas the answers of the second category served as predictor variable.

To test the dependency of the category Target inside Mask from the category Expansion, a model was calculated with the responses of Target inside Mask as criterion variable and the responses of Expansion as predictor. To test the dependency of the category Expansion from the category Target inside Mask, a model was calculated with the responses of Expansion as criterion variable and the responses of Target inside Mask as predictor. As fixed effects, Congruency as 2-level factor (congruent, incongruent), SOA as 6-level factor $(24,26,48,60,72,84 \mathrm{~ms})$ and the answer of the second Category as 2-level factor (yes, no) were used. All of the three predictors were effect-coded in the following way: the answer of respective other category: no $=0$, yes $=1$; Congruency: congruent $=0$, incongruent $=1$; SOA: $24 \mathrm{~ms}=1,36 \mathrm{~ms}=2,48 \mathrm{~ms}=3,60 \mathrm{~ms}=4,72 \mathrm{~ms}=5$, whereas the $84 \mathrm{~ms}$ SOA served as 
reference category. Subjects served as random effect on the intercept. In the same way two generalized linear mixed-effect regression models were calculated for the categories Rotation and Target before Mask. The only difference was the coding scheme for SOA. Because these models should focus on the dependency of both categories at long SOAs, the factor SOA was effect-coded as follows: $84 \mathrm{~ms}=1,72 \mathrm{~ms}=2,60 \mathrm{~ms}=3,48 \mathrm{~ms}=4,36 \mathrm{~ms}=5$, whereas the $24 \mathrm{~ms}$ SOA served as reference category.

All analyses started with the intercept-only model, a random effect on the intercept for participants. The fixed effects were added incrementally starting with the main effects SOA (model 1), Congruency (model 2) and Category 2 (model 3), followed by the two-way interactions between SOA x Congruency (model 4), SOA x Category 2 (model 5) and Congruency $\mathrm{x}$ Category 2 (model 6), at least the three-way interaction between SOA $x$ Congruency x Category 2 (model 7) was added. Chi-square tests on the log-likelihood values were used to compare the eight models and to conclude whether the added predictors improve the model fit.

\subsection{Results}

\subsubsection{Dependency of Expansion from Target inside Mask}

To consider if the probability to perceive an Expansion depended on SOA, Congruency and the perception of a Target inside Mask, a generalized linear mixed regression model was calculated. The inclusion of effects improved the model fit successively up to model 6 , from the intercept-only model to model $1\left(X^{2}(5)=1851.06, p<.001\right)$, from model 1 to model $2\left(X^{2}(1)=47.63, p<.001\right)$, from model 2 to model $3\left(X^{2}(1)=1755.98, p<.001\right)$, from model 3 to model $4\left(X^{2}(5)=29.34, p<.001\right)$, from model 4 to model $5\left(X^{2}(5)=11.22\right.$, $p=.047)$ and from model 5 to model $6\left(X^{2}(1)=4.88, p=.027\right)$. But the three-way interaction of model $7 \mathrm{did}$ not result in an improvement compared to model $6\left(X^{2}(5)=7.77, p=.17\right)$. Therefore, model 6 which included all main effects and all two-way interactions, showed the best fit. Figure 4.1A visualizes the conditional relative frequencies to report an Expansion for each level of SOA, Congruency, either under the condition that a Target inside Mask was negated or affirmed. Consequently, the frequencies of both conditions do not sum up to 1 . Figure 4.1B visualizes the odds ratios of model 6 . The parameters of model 6 can be extracted from Table 4.1. The letter "E" (Expansion) with the respective number of Table 4.1 refers to the coefficients of the model. 
4 | Phenomenological evidence for multiple processes underlying metacontrast masking

\section{Table 4.1}

Summary of the generalized linear mixed effect model, calculated for Expansion $(N=24$, Number of observations: 24192).

\begin{tabular}{lccccc}
\hline \multicolumn{1}{c}{ Expansion } & B & SE & $\boldsymbol{z}$ & OR & $p$-value \\
\hline Random effect of subject: variance $=0.93(S D=0.97)$ & & & & \\
1a. SOA $24 \mathrm{~ms}$ & 1.04 & 0.04 & 26.68 & 2.82 & $<.001$ \\
1b. SOA $36 \mathrm{~ms}$ & 0.35 & 0.04 & 9.74 & 1.42 & $<.001$ \\
1c. SOA $48 \mathrm{~ms}$ & -0.16 & 0.04 & -4.24 & 0.85 & $<.001$ \\
1d. SOA $60 \mathrm{~ms}$ & -0.40 & 0.04 & -10.03 & 0.67 & $<.001$ \\
1e. SOA $72 \mathrm{~ms}$ & -0.50 & 0.04 & -12.29 & 0.61 & $<.001$ \\
2. Congruency & 0.09 & 0.02 & 5.52 & 1.10 & $<.001$ \\
3. Target inside Mask & -0.73 & 0.02 & -39.95 & 0.48 & $<.001$ \\
4a. SOA $24 \mathrm{~ms} *$ Congruency & 0.09 & 0.03 & 2.56 & 1.09 & .01 \\
4b. SOA $36 \mathrm{~ms} *$ Congruency & 0.11 & 0.03 & 3.08 & 1.11 & .002 \\
4c. SOA $48 \mathrm{~ms} *$ Congruency & 0.04 & 0.04 & 1.24 & 1.05 & .21 \\
4d. SOA $60 \mathrm{~ms} *$ Congruency & -0.04 & 0.04 & -1.19 & 0.96 & .23 \\
4e. SOA $72 \mathrm{~ms} *$ Congruency & -0.07 & 0.04 & -1.81 & 0.93 & .07 \\
5a. SOA $24 \mathrm{~ms} *$ Target inside Mask & -0.04 & 0.04 & -1.05 & 0.96 & .29 \\
5b. SOA $36 \mathrm{~ms} *$ Target inside Mask & 0.03 & 0.04 & 0.75 & 1.03 & .45 \\
5c. SOA $48 \mathrm{~ms} *$ Target inside Mask & -0.05 & 0.04 & -1.20 & 0.95 & .23 \\
5d. SOA $60 \mathrm{~ms} *$ Target inside Mask & -0.03 & 0.04 & -0.65 & 0.97 & .51 \\
5e. SOA 72 ms * Target inside Mask & -0.03 & 0.04 & -0.73 & 0.97 & .46 \\
6. Congruency * Target inside Mask & -0.04 & 0.02 & -2.21 & 0.96 & .03 \\
\hline
\end{tabular}

Notes: Predictors were coded as follows: Target inside Mask: no $=0$, yes $=1$; Congruency: congruent $=0$, incongruent $=1 ; \mathrm{SOA}: 24 \mathrm{~ms}=1,36 \mathrm{~ms}=2,48 \mathrm{~ms}=3,60 \mathrm{~ms}=4,72 \mathrm{~ms}=5$, $84 \mathrm{~ms}=6$ (reference category); OR $=$ odds ratios. 


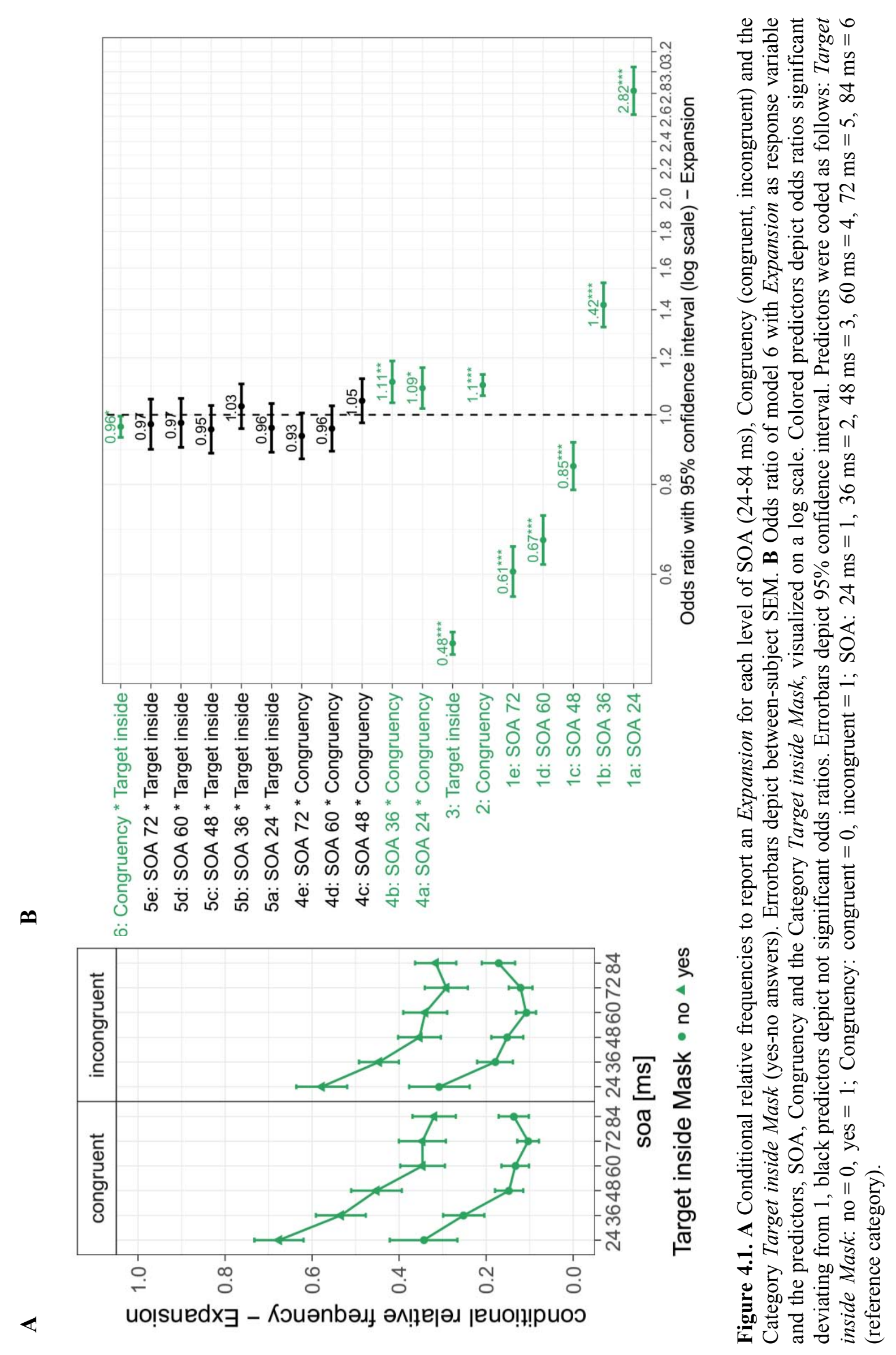


4 | Phenomenological evidence for multiple processes underlying metacontrast masking

The odds ratios declined successively from shortest to longest SOA, with odds ratios above 1 for the two shortest SOAs (E1a-b) and odds ratios below 1 for the other SOAs (E1ce). This showed that the probability to affirm Expansion decreased with SOA. On congruent trials the probability to affirm Expansion was slightly higher, compared to incongruent trials (E2). The main effect of Target inside Mask (E3) indicated that in trials where Target inside Mask was negated, the probability to affirm Expansion was less compared to trials where Target inside Mask has been affirmed. This shows that the perception of an Expansion was linked to the perception of a Target inside Mask. This result is important since it indicates that the perception of an Expansion depended on perception of a Target inside Mask. At 24 and $36 \mathrm{~ms} \mathrm{SOA}$ an interaction with Congruency was found (E4a-b) with odds ratios larger than 1. Even if no other significant interactions between the longer SOAs and Congruency could be found, the odds ratios successively decreased with SOA from values larger than 1 to values lower than 1 (E4c-e). This pattern indicated that at short SOAs the probability to perceive an Expansion was enhanced at congruent trials, whereas at long SOAs the revers tendency could be found with slightly more reports of an Expansion at incongruent trials. No significant interaction effect between SOA and Target inside Mask was found (E5), indicating that the dependency of the perception of an Expansion on the perception of a Target inside Mask was constant across SOA. An interaction effect between Congruency and Target inside Mask was found (E6).

\subsubsection{Dependency of Target inside Mask from Expansion}

To consider if the probability to perceive a Target inside Mask depended on SOA, Congruency or the perception of an Expansion, a generalized linear mixed regression model was calculated. From the intercept-only model to model $1\left(X^{2}(5)=1382.62, p<.001\right)$, the fit improved significantly, but from model 1 to model $2\left(X^{2}(1)=1.51, p=.22\right)$ the model fit did not improve. The inclusion of the other effects improved the model fit successively, from model 2 to model $3\left(X^{2}(1)=1638.38, p<.001\right)$, from model 3 to model $4\left(X^{2}(5)=24.66\right.$, $p<.001)$, from model 4 to model $5\left(X^{2}(5)=32.41, p<.001\right)$, from model 5 to model 6 $\left(X^{2}(1)=14.85, p<.001\right)$. But the three-way interaction of model 7 did not result in an improvement compared to model $6\left(X^{2}(5)=8.85, p=.12\right)$. Since the model fit significantly improved with the inclusion of interaction effects of Congruency (model 3 to model 4; model 5 to model 6), the main effect of Congruency must be included, even if it has not significantly improved the model fit significantly (model 1 to model 2). 


\section{Table 4.2}

Summary of the generalized linear mixed effect model, calculated for Target inside Mask $(N=24$, Number of observations: 24192).

\begin{tabular}{lccccc}
\hline \multicolumn{1}{c}{ Target inside Mask } & B & SE & $\boldsymbol{z}$ & OR & $\boldsymbol{p}$-value \\
\hline Random effect of subject: variance $=0.81$ & $(S D=0.9)$ & & & & \\
1a. SOA $24 \mathrm{~ms}$ & 0.88 & 0.04 & 23.13 & 2.43 & $<.001$ \\
1b. SOA $36 \mathrm{~ms}$ & -0.05 & 0.04 & -1.33 & 0.95 & .18 \\
1c. SOA $48 \mathrm{~ms}$ & -0.15 & 0.04 & -3.90 & 0.86 & $<.001$ \\
1d. SOA $60 \mathrm{~ms}$ & -0.21 & 0.04 & -5.13 & 0.81 & $<.001$ \\
1e. SOA $72 \mathrm{~ms}$ & -0.15 & 0.04 & -3.56 & 0.86 & $<.001$ \\
2. Congruency & -0.02 & 0.02 & -1.23 & 0.98 & .22 \\
3. Expansion & -0.71 & 0.02 & -38.54 & 0.49 & $<.001$ \\
4a. SOA $24 \mathrm{~ms} *$ Congruency & -0.05 & 0.04 & -1.32 & 0.95 & .19 \\
4b. SOA $36 \mathrm{~ms} *$ Congruency & -0.15 & 0.03 & -4.43 & 0.86 & $<.001$ \\
4c. SOA $48 \mathrm{~ms} *$ Congruency & -0.01 & 0.03 & -0.34 & 0.99 & .73 \\
4d. SOA $60 \mathrm{~ms} *$ Congruency & 0.05 & 0.03 & 1.63 & 1.05 & .10 \\
4e. SOA $72 \mathrm{~ms} *$ Congruency & 0.08 & 0.03 & 2.37 & 1.08 & .02 \\
5a. SOA $24 \mathrm{~ms} *$ Expansion & 0.15 & 0.04 & 3.80 & 1.16 & $<.001$ \\
5b. SOA $36 \mathrm{~ms} *$ Expansion & -0.03 & 0.04 & -0.94 & 0.97 & .35 \\
5c. SOA $48 \mathrm{~ms} *$ Expansion & -0.11 & 0.04 & -2.92 & 0.89 & .004 \\
5d. SOA $60 \mathrm{~ms} *$ Expansion & -0.07 & 0.04 & -1.82 & 0.93 & .07 \\
5e. SOA 72 ms * Expansion & -0.04 & 0.04 & -1.04 & 0.96 & .30 \\
6. Congruency * Expansion & -0.07 & 0.02 & -3.86 & 0.94 & $<.001$ \\
\hline
\end{tabular}

Notes: Predictors were coded as follows: Expansion: no $=0$, yes $=1$; Congruency: congruent $=0$, incongruent $=1 ; \mathrm{SOA}: 24 \mathrm{~ms}=1,36 \mathrm{~ms}=2,48 \mathrm{~ms}=3,60 \mathrm{~ms}=4,72 \mathrm{~ms}=5$, $84 \mathrm{~ms}=6$ (reference category); OR $=$ odds ratios. 


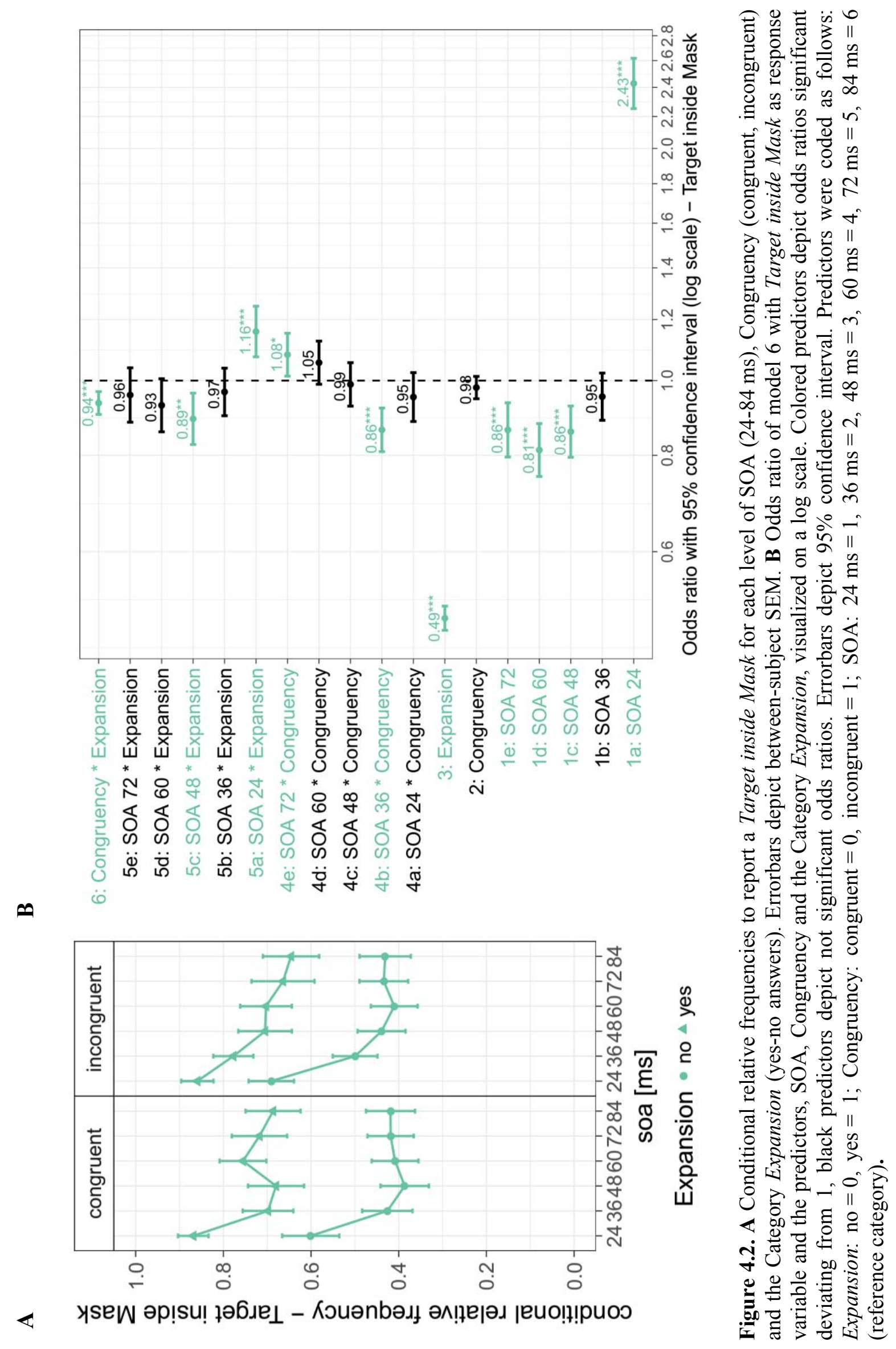


Figure 4.2A visualizes the conditional relative frequencies to report a Target inside Mask. Figure 4.2B visualizes the odds ratios of model 6. The parameters of model 6 can be extracted from Table 4.2. The letter "I" (Inside) with the respective number of Table 4.2 refers to the coefficients of the model.

The odds ratios were highest at the shortest SOA (I1a) and declined with increasing SOA (I1b-c) with a slight increase at $72 \mathrm{~ms} \mathrm{SOA} \mathrm{(I1d).} \mathrm{No} \mathrm{main} \mathrm{effect} \mathrm{of} \mathrm{Congruency} \mathrm{(I2)}$ was found. In accordance with the hypothesis a main effect of Expansion was found (I3). This effect corresponded with the result of the previous model and shows the interdependence of both perceptions. Interaction effects between SOA and Congruency were found with odds ratios increasing curvilinear with SOA (I4). At $36 \mathrm{~ms}$ SOA the odd ratio was significant below 1 (I4b) and rose until at $72 \mathrm{~ms}$ SOA a significant effect in the other direction was found (I4c-e). This pattern showed that at short SOA $(36 \mathrm{~ms})$ the probability to perceive a Target inside Mask was enhanced at incongruent trials, whereas at long SOA (72 ms) at congruent trials more often a Target inside Mask was perceived. Two significant interactions between SOA and Expansion were found (I5). At $24 \mathrm{~ms}$ SOA the differences in probability to affirm Target inside Mask between trials where an Expansion has been affirmed or negated was reduced, compared the mean of all SOAs (I5a). This indicated that the dependency of Target inside Mask on Expansion is less at $24 \mathrm{~ms}$ SOA, compared to the mean of the other SOAs. At $48 \mathrm{~ms} \mathrm{SOA}$ the reverse effect was found, indicating that the dependency of Target inside Mask on Expansion was more pronounced (I5c). Also an interaction between Congruency and Expansion was found (I6).

\subsubsection{Independency of Rotation from Target before Mask}

To consider if the probability to perceive a Rotation depended on SOA, Congruency or the perception of Target before Mask, a generalized linear mixed regression model was calculated. The inclusion of effects improved the model fit successively up to model 6 , from the intercept-only model to model $1\left(X^{2}(5)=371.62, p<.001\right)$, from model 1 to model 2 $\left(X^{2}(1)=3437.72, p<.001\right)$, from model 2 to model $3\left(X^{2}(1)=90.98, p<.001\right)$, from model 3 to model $4\left(X^{2}(5)=285.65, p<.001\right)$, from model 4 to model $5\left(X^{2}(5)=32.19, p<.001\right)$ and from model 5 to model $6\left(X^{2}(1)=39.17, p<.001\right)$. But the three-way interaction of model 7 did not result in an improvement compared to model $6\left(X^{2}(5)=5.19, p=.39\right)$. Therefore, model 6 which included all main effects and all two-way interactions, showed the best fit. 
4 | Phenomenological evidence for multiple processes underlying metacontrast masking

\section{Table 4.3}

Summary of the generalized linear mixed effect model, calculated for Rotation $(N=24$, Number of observations: 24192).

\begin{tabular}{lccccc}
\hline \multicolumn{1}{c}{ Rotation } & B & SE & $\boldsymbol{z}$ & OR & $\boldsymbol{p}$-value \\
\hline Random effect of subject: variance $=2.22$ & $(S D=1.49)$ & & & \\
1a. SOA $84 \mathrm{~ms}$ & 0.16 & 0.05 & 3.31 & 1.17 & $<.001$ \\
1b. SOA $72 \mathrm{~ms}$ & 0.26 & 0.04 & 5.94 & 1.3 & $<.001$ \\
1c. SOA $60 \mathrm{~ms}$ & 0.20 & 0.04 & 4.56 & 1.21 & $<.001$ \\
1d. SOA $48 \mathrm{~ms}$ & 0.01 & 0.04 & 0.26 & 1.01 & .80 \\
1e. SOA $36 \mathrm{~ms}$ & -0.17 & 0.04 & -4.03 & 0.84 & $<.001$ \\
2. Congruency & -1.01 & 0.02 & -51.03 & 0.36 & $<.001$ \\
3. Target before Mask & -0.23 & 0.02 & -10.82 & 0.79 & $<.001$ \\
4a. SOA $84 \mathrm{~ms} *$ Congruency & -0.31 & 0.04 & -7.28 & 0.73 & $<.001$ \\
4b. SOA $72 \mathrm{~ms} *$ Congruency & -0.28 & 0.04 & -6.76 & 0.75 & $<.001$ \\
4c. SOA $60 \mathrm{~ms} *$ Congruency & -0.18 & 0.04 & -4.25 & 0.84 & $<.001$ \\
4d. SOA $48 \mathrm{~ms} *$ Congruency & -0.09 & 0.04 & -2.15 & 0.91 & .03 \\
4e. SOA $36 \mathrm{~ms} *$ Congruency & 0.21 & 0.04 & 4.89 & 1.23 & $<.001$ \\
5a. SOA $84 \mathrm{~ms} *$ Target before Mask & 0.11 & 0.04 & 2.56 & 1.12 & .01 \\
5b. SOA 72 ms * Target before Mask & 0.10 & 0.04 & 2.34 & 1.10 & .02 \\
5c. SOA $60 \mathrm{~ms} *$ Target before Mask & 0.01 & 0.04 & 0.34 & 1.01 & .73 \\
5d. SOA $48 \mathrm{~ms} *$ Target before Mask & 0.002 & 0.04 & 0.07 & 1.00 & .94 \\
5e. SOA $36 \mathrm{~ms} *$ Target before Mask & -0.02 & 0.04 & -0.61 & 0.98 & .54 \\
6. Congruency * Target before Mask & -0.12 & 0.02 & -6.22 & 0.88 & $<.001$ \\
\hline
\end{tabular}

Notes: Predictors were coded as follows: Target before Mask: no $=0$, yes $=1$; Congruency: congruent $=0$, incongruent $=1$; SOA: $84 \mathrm{~ms}=1,72 \mathrm{~ms}=2,60 \mathrm{~ms}=3,48 \mathrm{~ms}=4,36 \mathrm{~ms}=5$, $24 \mathrm{~ms}=6$ (reference category); OR $=$ odds ratios. 


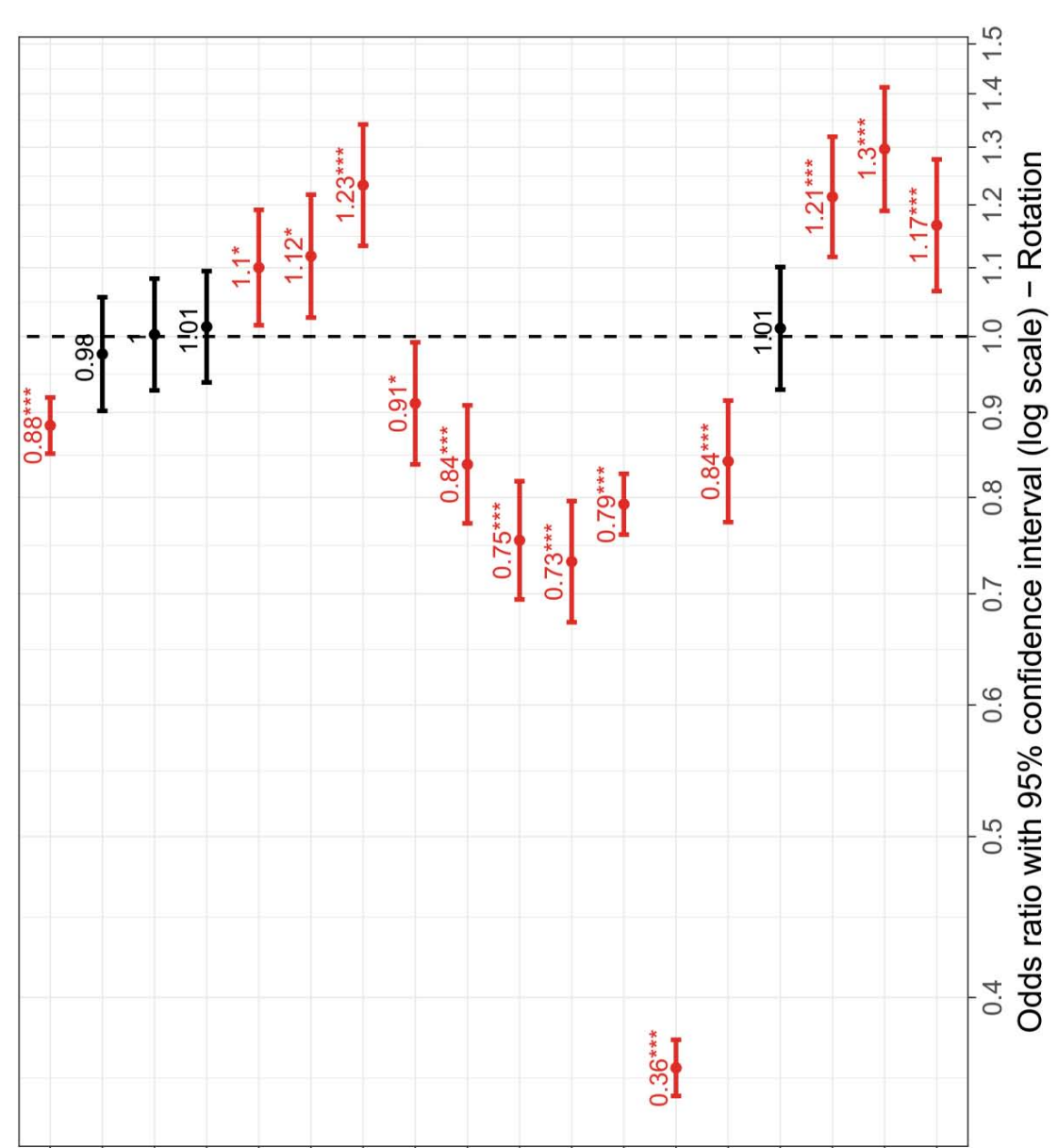

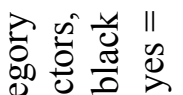

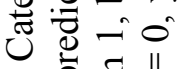

范

‡

可

言密

跣

粉

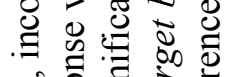

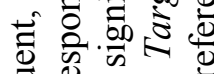

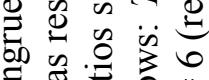

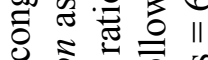

这

বे 형

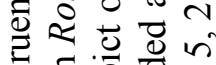

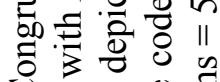

0 o

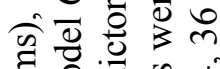

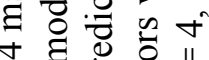

क

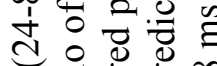

局它完

o 0 ठ

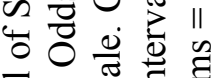

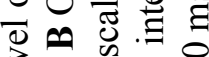

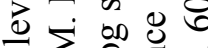

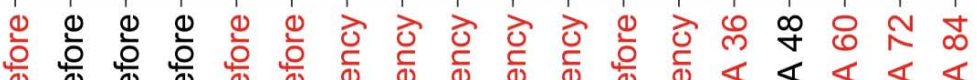

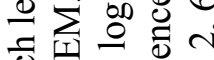

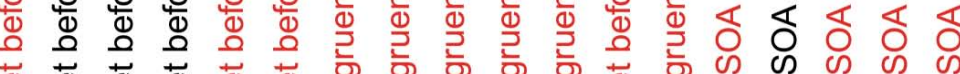

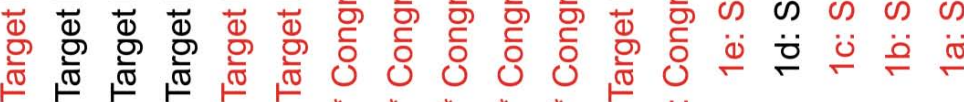

* * * * * * * *

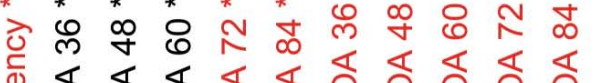

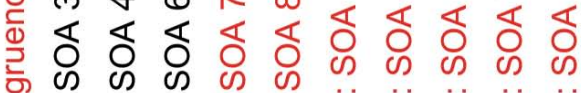

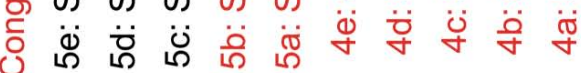

$\infty \quad \ddot{0}$

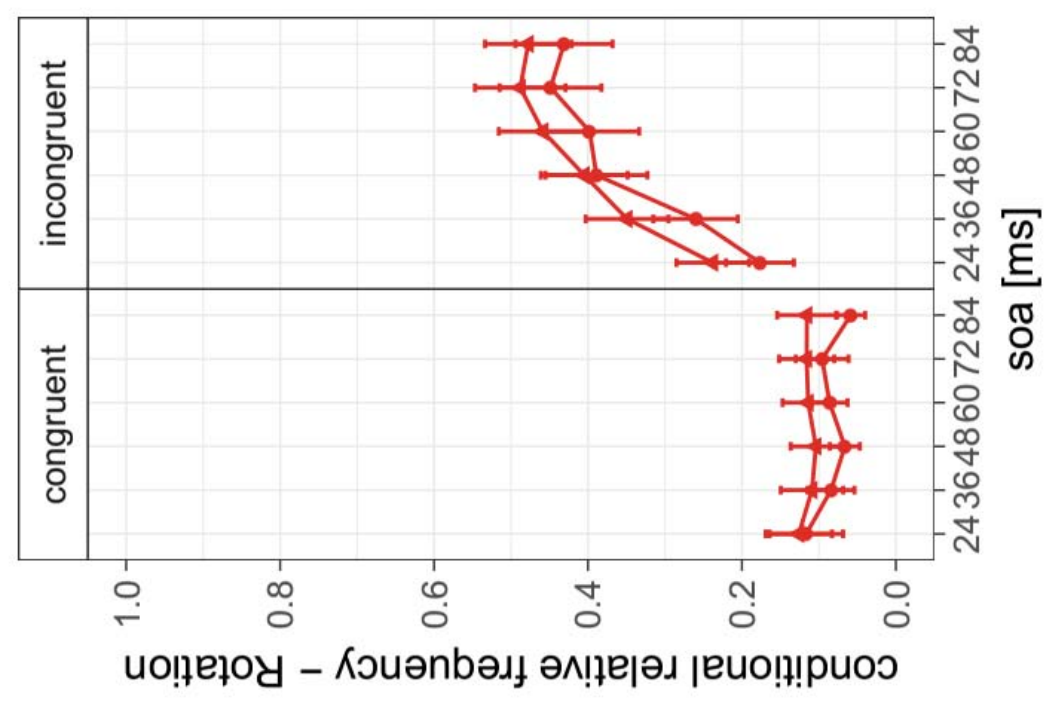

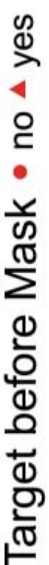

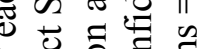

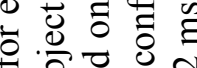

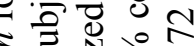

춘

ष $\overrightarrow{0}$ ।

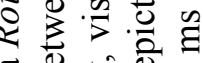

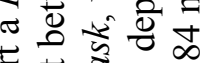

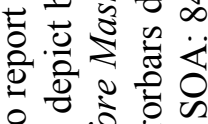

के

政 \&

过

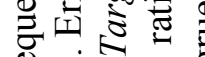

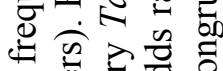

0 定

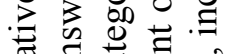

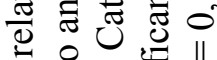

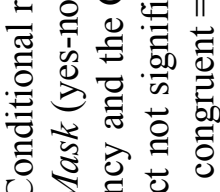

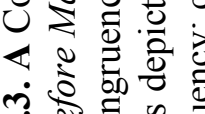

भi

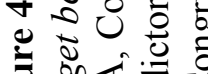

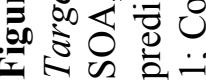


4 | Phenomenological evidence for multiple processes underlying metacontrast masking

Figure 4.3A visualizes the conditional relative frequencies to report a Rotation, Figure 4.3B visualizes the odds ratios of model 6 . The parameters of model 6 can be extracted from Table 4.3. The letter "R" (Rotation) with the respective number of Table 4.3 refers to the coefficients of the model.

The probability to affirm Rotation increased in a slight revers $\mathrm{u}$-shaped manner with SOA (R1). At $84 \mathrm{~ms}$ SOA the probability to affirm Rotation was higher, compared to the mean of all SOAs (R1a). At $72 \mathrm{~ms}$ SOA the odds ratio for Rotation even rose (R1b) and decreased again with decreasing SOA (R1c-e). On congruent trials the probability of affirming a Rotation was lower than on incongruent trials (R2). A main effect of Target before Mask was found (R3), indicating that the probability to affirm Rotation depended on the perception of a Target before Mask. But to anticipate, in addition two significant interaction effects between SOA and Target before Mask were found (R5). Compared to the average of all SOAs, at $84 \mathrm{~ms}$ SOA the probability of perceiving a Rotation differed less between trials on which a Target before Mask was negated or affirmed (R5a). The same was visible for the $72 \mathrm{~ms}$ SOA (R5b). This indicated that the dependency of Rotation on the perception of a Target before Mask was reduced at long SOAs. For the other SOAs no significant interaction with Target before Mask was found (R5c-e). Therefore, for these SOAs the dependency of Rotation on the perception of a Target before Mask did not differ from the general dependency, visible in the main effect of Target before Mask (R3). To sum, even if the main effect of Target before Mask showed a dependency between both perceptions, the significant interaction between Target before Mask and the 72 and $84 \mathrm{~ms}$ SOAs indicated that this dependency was reduced at long SOAs. All interaction effects between SOA and Congruency were significant (R4). At $84 \mathrm{~ms}$ SOA, compared to the mean of all SOA, the difference in probability to affirm Rotation between congruent and incongruent trials was enhanced (R4a). With decreasing SOA the odds ratios for the interaction between SOA and Congruency gradually approached the 1 , until at $36 \mathrm{~ms}$ SOA an odds ratio greater than 1 was found. This indicated that the difference in probability to affirm Rotation between congruent and incongruent trials was reduced at $36 \mathrm{~ms} \mathrm{SOA}$, compared to the mean of all SOAs. Also the interaction between Congruency and Target before Mask was significant (R6). Thus, compared to incongruent trials at congruent trials the differences in probability to affirm a Rotation between trials on which a Target before Mask was negated or affirmed was enhanced. This indicated that the dependency of Rotation on the perception of a Target before Mask was higher on congruent, compared to incongruent trials. 


\section{Table 4.4}

Summary of the generalized linear mixed effect model, calculated for Target before Mask $(N=24$, Number of observations: 24192).

\begin{tabular}{|c|c|c|c|c|c|}
\hline Target before Mask & B & SE & $z$ & OR & $p$-value \\
\hline \multicolumn{6}{|c|}{ Random effect of subject: variance $=1.36(S D=1.17)$} \\
\hline 1a. SOA $84 \mathrm{~ms}$ & 0.9 & 0.04 & 22.85 & 2.46 & $<.001$ \\
\hline 1b. SOA $72 \mathrm{~ms}$ & 0.49 & 0.04 & 13.03 & 1.63 & $<.001$ \\
\hline 1c. SOA $60 \mathrm{~ms}$ & 0.06 & 0.04 & 1.54 & 1.06 & .12 \\
\hline 1d. SOA $48 \mathrm{~ms}$ & -0.40 & 0.04 & -10.50 & 0.67 & $<.001$ \\
\hline 1e. SOA $36 \mathrm{~ms}$ & -0.63 & 0.04 & -15.64 & 0.53 & $<.001$ \\
\hline 2. Congruency & -0.02 & 0.02 & -1.16 & 0.98 & .24 \\
\hline 3. Rotation & -0.21 & 0.02 & -9.40 & 0.81 & $<.001$ \\
\hline 4a. SOA $84 \mathrm{~ms} *$ Congruency & -0.01 & 0.04 & -0.30 & 0.99 & .76 \\
\hline 4b. SOA $72 \mathrm{~ms} *$ Congruency & -0.02 & 0.04 & -0.42 & 0.98 & .68 \\
\hline 4c. SOA $60 \mathrm{~ms} *$ Congruency & -0.05 & 0.04 & -1.31 & 0.95 & .19 \\
\hline 4d. SOA $48 \mathrm{~ms} *$ Congruency & -0.05 & 0.04 & -1.46 & 0.95 & .14 \\
\hline 4e. SOA $36 \mathrm{~ms} *$ Congruency & 0.12 & 0.03 & 3.58 & 1.13 & $<.001$ \\
\hline 5a. SOA $84 \mathrm{~ms} *$ Rotation & 0.20 & 0.04 & 4.77 & 1.23 & $<.001$ \\
\hline 5b. SOA $72 \mathrm{~ms} *$ Rotation & 0.17 & 0.04 & 4.12 & 1.18 & $<.001$ \\
\hline 5c. SOA $60 \mathrm{~ms} *$ Rotation & 0.04 & 0.04 & 0.92 & 1.04 & .36 \\
\hline 5d. SOA $48 \mathrm{~ms} *$ Rotation & -0.002 & 0.04 & -0.05 & 1.00 & .96 \\
\hline 5e. SOA $36 \mathrm{~ms} *$ Rotation & -0.09 & 0.04 & -2.12 & 0.92 & .03 \\
\hline 6. Congruency $*$ Rotation & -0.06 & 0.02 & -2.92 & 0.94 & .003 \\
\hline
\end{tabular}

Notes: Predictors were coded as follows: Rotation: no $=0$, yes $=1$; Congruency: congruent $=0$, incongruent $=1$; SOA: $84 \mathrm{~ms}=1,72 \mathrm{~ms}=2,60 \mathrm{~ms}=3,48 \mathrm{~ms}=4,36 \mathrm{~ms}=5$, $24 \mathrm{~ms}=6$ (reference category); OR $=$ odds ratios. 

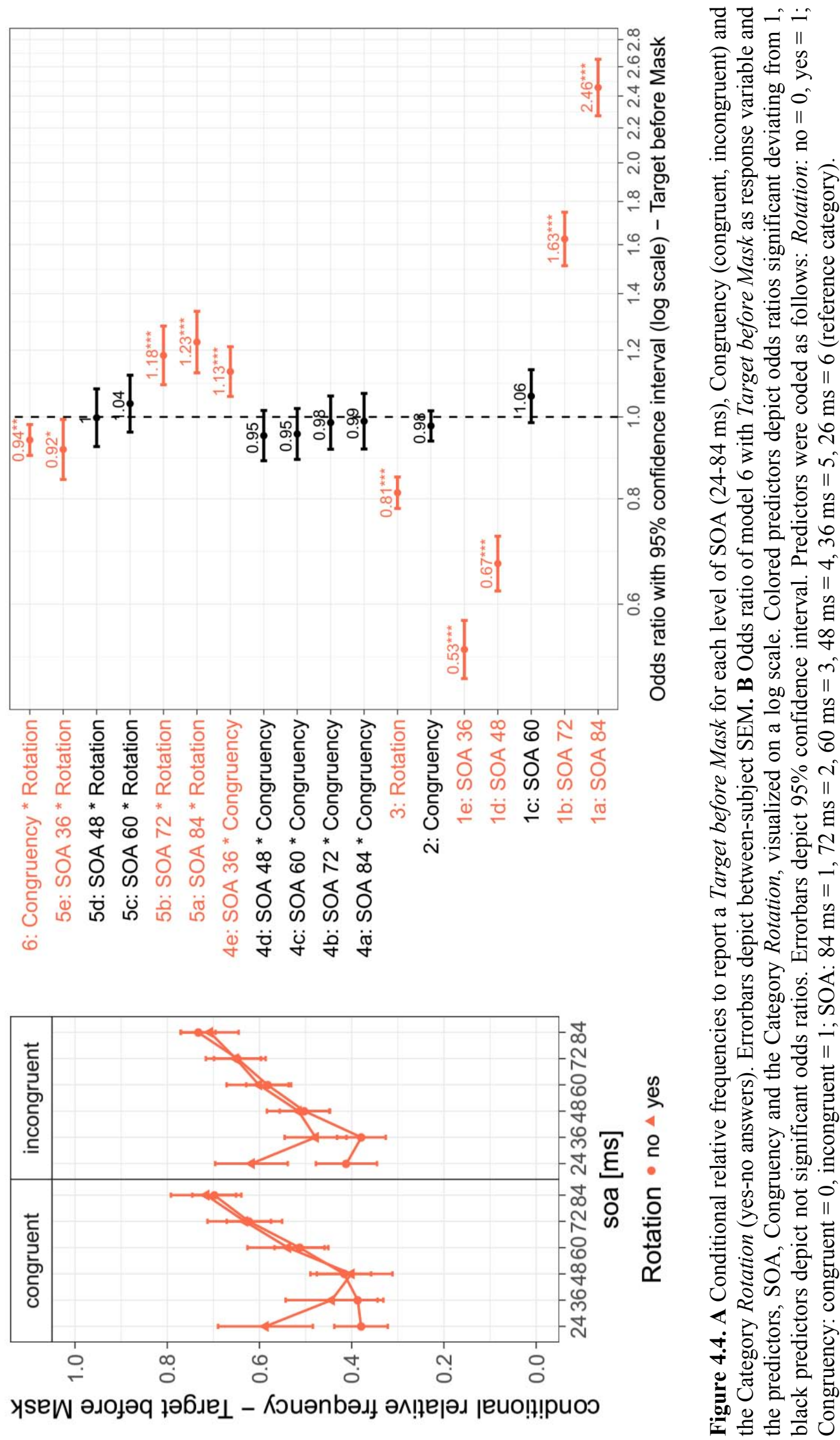

$\simeq$

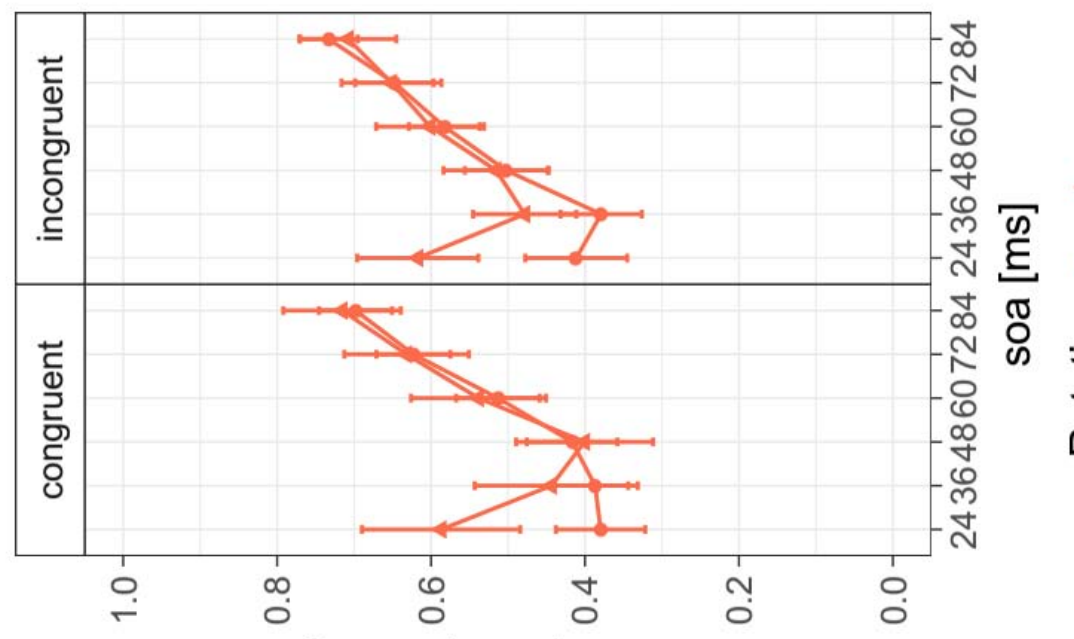

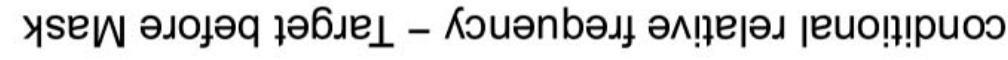




\subsubsection{Independency of Target before Mask from Rotation}

To consider if the probability to perceive a Target before Mask depended on SOA, Congruency or the perception of a Rotation, a generalized linear mixed regression model was calculated. The inclusion of effects improved the model fit successively up to model 6 , from the intercept-only model to model $1\left(X^{2}(5)=1802.44, p<.001\right)$, from model 1 to model 2 $\left(X^{2}(1)=46.06, p<.001\right)$, from model 2 to model $3\left(X^{2}(1)=73.58, p<.001\right)$, from model 3 to model $4\left(X^{2}(5)=16.19, p=.006\right)$, from model 4 to model $5\left(X^{2}(5)=80.89, p<.001\right)$, from model 5 to model $6\left(X^{2}(1)=8.56, p=.003\right)$. But the three-way interaction of model 7 did not result in an improvement compared to model $6\left(X^{2}(5)=1.63, p=.9\right)$.

Therefore, model 6 which included all main effects and all two-way interactions showed the best fit. Figure 4.4A visualizes the conditional relative frequencies to report a Target before Mask, Figure 4.4B visualizes the odds ratios of model 6. The parameters of model 6 can be extracted from Table 4.4. The letter "B" (Before) with the respective number of Table 4.4 refers to the coefficients of the model. The probability to affirm Target before Mask showed an increase with SOA (B1). At $84 \mathrm{~ms}$ SOA the odds ratio were highest (B1a), indicating that at $84 \mathrm{~ms}$ SOA the probability to perceive a Target before Mask was higher, compared to the mean of all SOAs. The odds ratios sunk with decreasing SOA (B1b-e), until the odds ratio of $36 \mathrm{~ms} \mathrm{SOA}$ was significantly below 1, indicating that at $36 \mathrm{~ms}$ SOA the probability to perceive a Target before Mask was lower, compared to the mean of all SOAs. No effect of Congruency was found (B2). A main effect of Rotation was found (B3), indicating that the perception of a Target before Mask depended on the perception of a Rotation. But as in the previous model, significant interaction effects between SOA and Rotation were found (B5). At $84 \mathrm{~ms} \mathrm{SOA}$, compared to the mean of all SOAs, the differences in probability to affirm Target before Mask between trials where Rotation has been negated or affirmed was reduced (B5a). The same was visible at $72 \mathrm{~ms}$ SOA (B5b). In general, the odds ratios for the interaction between SOA and Rotation decreased with decreasing SOA, until at $36 \mathrm{~ms}$ SOA the reverse effect was found (B5c-e). The reverse effect indicated that the dependency of Target before Mask on the perception of a Rotation was enhanced at $36 \mathrm{~ms}$ SOA. In accordance with the previous model did this pattern show that the dependency between both perceptions was reduced at long SOAs (72 and $84 \mathrm{~ms}$ ). At most SOAs no interaction with Congruency was found (B4a-d), except at $36 \mathrm{~ms}$ SOA (B4e). Also an interaction between Congruency and Rotation was found (B6). 
4 | Phenomenological evidence for multiple processes underlying metacontrast masking

\subsection{Discussion}

\subsubsection{Evidence for one processes underlying short SOAs}

To consider the relationship between the categories Expansion and Target inside Mask two models were calculated, which examined whether the affirmation of one category depended on SOA, Congruency and the affirmation of the respective other category. Both models confirmed the decreasing time course across SOA of both perceptions, depict in Experiment 2 of this thesis. Based on the assumption of two-process theories (Neumann \& Scharlau, 2007; Reeves, 1982) and the results of Albrecht and Mattler (2016) it is reasonable to assume metacontrast as a multidimensional phenomenon. The temporal parameter, more precisely the SOA, determines which underlying process modulates the target visibility. Thus, the temporal parameter seems to have a deterministic influence on the emergence of the underlying process. This was also supported by the results of the present experiment. The odd to perceive an Expansion at $24 \mathrm{~ms}$ SOA was 2.82 time higher compared to the mean of all SOAs and the odd to perceive a Target inside Mask at 24 ms SOA was 2.42 time higher compared to the mean of all SOAs. This shows the high significance of the temporal parameter, more precisely the SOA, for the occurrence of both perceptions. Regarding the dependency of the perceptions on each other, both models showed nearly identical odds ratios for the respective other category. For Expansion the dependency on the perception of a Target inside Mask was constant across SOA. For Target inside Mask the dependency with Expansion was slightly reduced at shortest SOA, but still existent. Therefore, it can be concluded that there is a reciprocal dependence between the perception of an Expansion and a Target inside Mask. These results support the assumption of Albrecht and Mattler (2016) that one process is sufficient to explain the variety of the target perception at short SOAs. This process elicits two qualitative different perceptions and shows the largest impact on the target perception at short SOAs which declines with increasing temporal distance. Some authors differentiated between a boundary and surface completion process both underlying short SOAs (Bachmann, 2009b; Breitmeyer et al., 2006; Paradiso \& Nakayama, 1991; Stoper \& Mansfield, 1978). Since perceptions relating to one of these processes were not investigated in the present investigation, it cannot be excluded with certainty that differences between a boundary and a surface completion process exist. However, the results show that, despite the consideration of different perceptions, the results of Albrecht and Mattler (2016) could be replicated. 
Nevertheless, the conclusion about one underlying process must be treated with caution since one category was also negated in trials on which the other category has been affirmed and vice versa. Consequently, even if a mutual dependency has been found that suggested that both perceptions were linked with the same process, it cannot be conclusively concluded that a uniform process is associated with both perceptions. Instead, there are several possibilities to explain how the interrelated perceptions may be linked to underlying processes. These explanations will be discussed below.

First, the dependency of both perceptions could result from trial-by-trial variations in the ability to perceive the target, due to attention fluctuations or due to noise inherent to neural processing (Wiens, 2008). Consequently, in trials where the visual system is disturbed in processing the stimulus, both perceptions would be less likely to be perceived. Based on this explanation, both perceptions are not elicited by a perceptual process, but depend on a general mechanism of visual processing. The following two reasons speak against this explanation. If the perception is characterized by such a general mechanism, this mechanism should be generalized to any other perception. Thus, a dependency between the categories Target before Mask and Rotation should also be found. But instead, these categories were found to be independent of each other. Consequently, the dependency found for the perception of an expanding target and a target integrated inside the mask is unlikely to be attributed to a general processing mechanism. Even a kind of perceptional bias can be invalidated with this argumentation. If the perception of one category would automatically lead to the conclusion that the other category must also have been visible, it remains unclear why this pattern was not found for the other categories, Target before Mask and Rotation.

Second, instead of one process, there could be two underlying processes, one of which serving as the prerequisite for the other. In this case, the perception elicited by the dependent processes should strongly depended on perception elicited by the preconditioning process. But the other way around no dependency should be visible. This explanation seems to be rather unlikely, since an asymmetric dependency between both perceptions should result, which is not the case. Instead, the dependency found for both models was nearly identical. This precludes considerations of two processes underlying metacontrast at short SOAs, with one process serving as requirement for the other process.

Third, both perceptions could be elicited by two independent processes, both strongly depending on the same experimental parameter, for example SOA. Due to the dependency of both processes on the same experimental variable, the impression of an apparent dependence of both perceptions could result. In order to investigate, if one or two processes are necessary 
4 | Phenomenological evidence for multiple processes underlying metacontrast masking

to explain the dependency of both perceptions, it should examine, if a dissociation of both experiences can be evoked. For this purpose, parameters should be manipulated which are known to influence the masking function at short SOAs. One could consider whether the manipulations affect both perceptions equally or whether a dissociation of both measures occurs (Schmidt \& Vorberg, 2006). The latter would provide evidence that both perceptions can be associated to different processes. However, if the experimental manipulations had an equal effect on the experience of both categories, this would support the presumption that both perceptions are elicited by one underlying process. At short SOAs the masking function is sensitive for stimulus features like variations of the mask luminance (Bridgeman, 2001), the target luminance (Francis, 1997), the spatial separation between target and mask (Growney et al., 1977; Kolers \& Rosner, 1960; Merikle, 1977) or the duration of the mask (Breitmeyer, 1978a; Neumann \& Scharlau, 2007). Thus, these manipulations are assumed to influence perceptions emerging at short SOAs and therefore are also suitable to investigate a possible dissociation. Based on the present results, neither the explanation of one process eliciting both perceptions, nor the assumption of two independent processes, both depending in a similar manner on an experimental variable, can be favored or excluded with certainty. But in the sense of favoring the simpler theory, the assumption of one process should be preferred, compared to two processes with the same time course.

The influence of Congruency on both perceptions seemed to be less clear for several reasons: First, the odds ratios of any effect of Congruency, including the effect of Congruency on the dependency of both perceptions, showed only little deviations from 1 . Therefore, the effect of Congruency was only marginal and much smaller compared to the effect of SOA. Second, the opposite direction of interaction effects between SOA and Congruency was found for both models. At short SOAs a Target inside Mask was more often perceived at incongruent trials, whereas an Expansion was perceived rather at congruent trials. At long SOAs this pattern reversed. A Target inside Mask was perceived more often at congruent trials, whereas a tendency was visible that an Expansion was more likely to be perceived at incongruent trials. Therefore, the spatial parameter seems to change only the probability which of the two perceptions is more likely to be perceived. But the spatial layout of the stimuli does not seem to have a deterministic influence on the emergence of the perceptions associated with short SOAs.

To sum up, the results support the assumption of one underlying process eliciting both perceptions, which is strongly modulated by the temporal parameter. The spatial parameter did not seem to have a deterministic influence on the emergence of the underlying process. 
Instead, the Congruency seemed to have an impact on the perceptual outcome of the process, by changing the probability which one of the two perceptions is more likely to be perceived. However, further research is necessary to support or falsify the assumption of one underlying process.

\subsubsection{Evidence for two processes underlying long SOAs}

To consider the relationship between the categories Rotation and Target before Mask two models were calculated, which examined whether the affirmation of one category depended on SOA, Congruency and the affirmations of the respective other category. Both categories yielded an increasing time course across SOA, supporting the results of Albrecht and Mattler (2016) and of Experiment 1-2 of this thesis. Further, the perception of a Rotation strongly depended on Congruency, with more affirmations of Rotation at incongruent trials. In addition, this effect increased with SOA. However, Congruency did not seem to influence the perception of a Target before Mask substantially. Consequently, Congruency had a deterministic influence on the perception of a Rotation, whereas the perception of a Target before Mask seemed to be independent of the spatial parameter. This can be interpreted as evidence for two independent processes underlying long SOAs, both associated with the perception of a different target aspect. In contrast, a dependency was found for both categories, whose effect size was equal for both models. However, the odds ratios for this effect deviate only slightly from 1. Furthermore, for both models an interaction between SOA and the respective other category was found, which indicated that with increasing SOA the dependency between both perceptions decreased. Consequently, at long SOAs both perceptions seem to be independent, which can be interpreted as evidence for two independent processes. In accordance with the assumption of Albrecht and Mattler (2016) these processes were associated with a rotational movement and a form independent visibility at long SOAs.

The effect of dependency found for the perception of an Expansion and a Target inside Mask showed that the odd to perceive one of these categories was about $50 \%$ smaller in trials were the respective other category has been negated, compared to trials were the other category has been affirmed. In contrast, the odd to perceive a Rotation or Target before Mask differed only about $20 \%$. Consequently, even if a dependency was found for all categories, the amount of dependency differed a lot between the two pairs of categories. 
4 | Phenomenological evidence for multiple processes underlying metacontrast masking

Nevertheless, a dependency, even if it was only very little in effect size, could not be excluded totally for the perceptions at long SOAs. Therefore, further investigations of a possible dissociation between both perceptions could provide convincing evidence for the assumption of independence. Studies that examine whether apparent motion and metacontrast can be attributed to the same underlying mechanism already provided parameters, which influenced both perceptions differently. Weisstein and Growney (1969) highlighted that the metacontrast function was sensitive to changes in the following parameters: spatial distance between target and mask, viewing conditions (monoptic, dichoptic) and stimulus duration and luminance.

First, variations of the spatial distance had a strong impact on the visibility at various SOAs for metacontrast masking (Alpern, 1953; for review see Breitmeyer \& Öğmen, 2006; Growney et al., 1977; Stigler, 1910; Werner, 1935). A direct comparison between apparent motion and metacontrast yielded that large spatial distance exclusively eliminated metacontrast, whereas the apparent motion was still perceivable (Breitmeyer \& Horman, 1981; Hein \& Moore, 2010b; Weisstein \& Growney, 1969).

Second, the effect of the spatial distance on the metacontrast function was even enhanced under dichoptic masking (Weisstein \& Growney, 1969). In contrast, the effect of the spatial distance on apparent motion did not differ between dichoptic and monoptic conditions (Weisstein \& Growney, 1969).

Third, with increasing luminance the u-shaped metacontrast function became more accentuated, whereas the luminance did not affect the apparent motion function (Weisstein \& Growney, 1969).

Fourth, for the smallest luminance with increasing stimulus duration the metacontrast masking decreased across the whole range of SOAs, but left the apparent motion intact (Weisstein \& Growney, 1969).

Fifth, at long SOAs the masking function depended on attention manipulations, for example the appearance of a distractor on the opposite side of the masking sequence (Neumann \& Scharlau, 2007) or the presentation of spatial flanker cues, guiding the attention to facilitate the target perception (Bruchmann, Hintze, \& Mota, 2011). Instead, for apparent motion the predictability of the location of the second flash had no influence on the subjective rating of the quality of the movement (Beck, Elsner, \& Silverstein, 1977).

Sixth, for small spatial distances the contrast polarity affected the apparent motion perception. But with increasing spatial distance, apparent motion became less dependent on the contrast polarity (Anstis \& Mather, 1985). The masking strength of the metacontrast was 
lower with stimuli of opposite polarity than with stimuli of the same polarity (Becker \& Anstis, 2004; Breitmeyer, 1978b; Breitmeyer, Tapia et al., 2008). These parameters allow to investigate whether the perception of a Rotation and Target before Mask show a dissociation. It can be assumed that these experimental manipulations should yield a dissociation between both categories, Target before Mask and Rotation. This would provide another evidence for independent processes underlying both perceptions.

\subsubsection{Assumptions about the type of processes underlying metacontrast masking at short and long SOAs}

Finally, it should be argued which types of processes are most likely to be associated with the perceptions at short and long SOAs. One approach to explain metacontrast masking relied solely on feedforward processing. These models defined lateral inhibition as a key mechanism of metacontrast masking, which means that neighboring cells inhibit each other via horizontal connections (Bridgeman, 1971, 2001; Francis, 1997; Macknik \& Livingstone, 1998; Macknik \& Martinez-Conde, 2004, 2007; Stigler, 1910; Weisstein, 1972; Weisstein \& Growney, 1969). Lateral inhibition models were able to simulate the influence of several variables, which affected the metacontrast function mostly at short SOAs. Francis's (1997) model simulated that the duration of target and mask influence the metacontrast function (Breitmeyer, 1978a; Merikle, 1977; Neumann \& Scharlau, 2007) and that inhibition affected the contrast at the border of the target more than at the center (Petry, 1978; Werner, 1935). The impact of the mask's contour (Sherrick \& Dember, 1970), was simulated by Bridgeman (1971, 2001) and Francis (1997). The effect of the luminance contrast of the stimuli, has been simulated by several models (Bridgeman, 1971, 2001; Francis, 1997; Weisstein, 1972). Therefore, it is likely that lateral inhibition is an appropriate mechanism to explain metacontrast masking effects at short SOAs.

Even the perceptions of an expanding target and a target integrated inside mask may be explained with a lateral inhibition model. The impression of an expansion could result from a misinterpretation of a contrast gradient between the center and the border of the target stimulus, resulting from lateral inhibition of the adjacent contours of target and mask (Petry, 1978; Werner, 1935). The brief, flash-like appearance of the stimulus, in combination with the gradient of the target's surface contrast, could be falsely perceived as an expansion. The perception of a target integrated inside the mask can be explained with the low temporal resolution of the visual system at short SOAs (Eriksen \& Rohrbauch, 1970), which does not 
4 | Phenomenological evidence for multiple processes underlying metacontrast masking

allow a temporal separation of target and mask (Francis \& Cho, 2008; Neumann \& Scharlau, 2007; Reeves, 1982). Since target and mask do not overlap spatially at metacontrast masking, the temporal integration helps to perceive the target (Francis \& Cho, 2008). Francis (1997) lateral inhibition model explained the high visibility at short SOAs, by the strength of the excitatory feedback of the target which is strong enough not to be suppressed by the inhibition of the mask. But with increasing SOA the excitatory feedback diminishes successively and the inhibitory activity of the mask has a stronger impact on the processing of the target. An earlier version of the model was also able to simulate the results of temporal integration experiments, inter alia, demonstrating a decrease in integration with increasing interstimulus interval (ISI) (Francis, 1996). Consequently, lateral inhibition mechanisms can explain both, the perception of a target integrated inside the mask, which seems to expand as a result of the contrast gradient from the center to the border of the target, as well as the decreasing time course of these perceptions with increasing SOA.

Another approach to explain metacontrast masking differentiated between two processes, underlying both branches of the metacontrast function. The process at short SOAs was assumed to be located at early processing stages and the second mechanism, responsible for the masking function at long SOAs, at a more central stage (Neumann \& Scharlau, 2007). A further development of metacontrast masking models which were based on electrophysiological findings of object processing (Bar, 2003; Fenske et al., 2006; for review see, Lamme \& Roelfsema, 2000), differentiated between feedforward and feedback, respectively reentrant mechanism (Di Lollo et al., 2000; Fahrenfort et al., 2007; Tapia \& Beck, 2014; Tapia \& Breitmeyer, 2011). These models proposed that the feedforward sweep of the target processing was left intact in backward masking paradigms, whereas feedback or recurrent processes were disrupted by the mask.

At short SOAs masking effects based on feedforward processing were explained by low-level contour interactions, whereas a disruption of reentrant processing was attributed to masking effects at long SOAs (Bachmann, 2015; Bridgeman, 1980; Di Lollo et al., 2000; Enns \& Di Lollo, 2000). Authors assuming high-level masking mechanisms at long SOAs proposed Object Substitution Masking (OSM) as a plausible masking mechanism. Even if OSM was original assigned to common-onset paradigms, with target and mask appearing with simultaneous onset, but delayed offset (Di Lollo et al., 2000), it has been generalized to other types of backward masking (Bachmann, 2005; Di Lollo et al., 2000; Enns \& Di Lollo, 2000). OSM was assumed to take place if the target was replaced by a mask, before the target information was identified (Enns, 2004). As a consequence the bottom-up processed 
information does not match with the reentrant signal, causing the representation of the target to be substituted by the mask (Di Lollo et al., 2000; Enns \& Di Lollo, 2000).

In the present experiment at long SOAs evidence for two independent perceptions was obtained, the perception of a rotational movement relating to apparent motion and the perception of a target temporally segregated from the mask reflecting a form independent target visibility at long SOAs. Multiple processes may be explained with independent masking of different stimulus features. The assumption that different object features are masked independently, is supported by object processing theories, which proposed multiple parallel feedforward sweeps processing different stimulus features at different speeds (Lamme \& Roelfsema, 2000; Livingstone \& Hubel, 1988; Zeki, 2015).

Also for OSM, stimulus features differed in their susceptibility to being integrated into the mask's presentation, depending on the target-mask dis-, similarity on the particular feature dimension (Gellatly, Pilling, Cole, \& Skarratt, 2006). Within an OSM framework, a rotation can be interpreted as the result of an incomplete segregation of target and mask. Instead, the target information is integrated within the representation of the mask. In accordance, Hein and Moore (2010b) concluded that apparent motion resulted from a failed individuation of the target, because the mask is falsely interpreted as later instantiations of the target and both stimuli are integrated inside one representation. This assumption has been supported by Pilling and Gellatly (2009), who showed that apparent motion in a standing wave illusion paradigm was enhanced if target and masks had similar forms, making it more likely that both stimuli would be misinterpreted as one object.

With diamond- and square-shaped stimuli, the substitution of a target form within the representation of the mask could result on incongruent trials to the perception of a rotational movement. In contrast, if the target is perceived temporally segregated from the mask, the target individuation has been completed and the information of both stimuli was processed independently. However, in the present investigation the perception of a temporally segregated target does neither imply a completed individuation, nor the perception of a motion can be equated with a phenomenally absent target, since a Rotation was perceived with and without perceiving a Target before Mask and vice versa. Instead, to perceive a Rotation it is necessary that the information about the contour of the target is mapped to the representation of the mask. To perceive the target as a temporally separated object, the target surface information must be processed independently of surface information of the mask. 
4 | Phenomenological evidence for multiple processes underlying metacontrast masking

\subsubsection{Conclusion}

In metacontrast masking the ability of the visual system to process the target information is impaired by the following mask. Despite the processing of the target is not impeded entirely. There is a residual phenomenology of the target, which is not constant, but varies qualitatively across SOA. In dependence of the temporal relation of target and mask different features of the target can or cannot be processed. The relationship of the temporal and even spatial parameter, found in Experiment 2 of this thesis, have mostly been confirmed by the results of this investigation. This is another validation for the percepts extracted from metacontrast literature. Again, the temporal parameter seems to have a deterministic influence on the processing of target features. Thus, different temporal signatures have been found for the two pairs of percepts which were associated with short and long SOAs, respectively.

At short SOAs a dependency between the categories Target inside Mask and Expansion has been found. In accordance with the results of Albrecht and Mattler (2016), it was interpreted as evidence for one mechanism underlying metacontrast masking at short SOAs. Because only behavioral results were provided by the present experiment, conclusions about underlying mechanisms can only be made with caution. However, lateral inhibition could be a possible mechanism to explain both perceptions at short SOAs. Lateral inhibition models were able to simulate the visibility of the target (Bridgeman, 1980; Francis, 1997; Weisstein, 1972), the temporal integration of target and mask (Francis, 1996) and the contrast gradient between the center and the border of the target (Francis, 1997), which may cause the perception of an expanding target. In contrast, at long SOAs an independence between both perceptions was found, which speaks in favor for the assumption that two processes underlie metacontrast masking at long SOAs (Albrecht \& Mattler, 2016).

At long SOAs high-level processes were proposed (Fahrenfort et al., 2007; Tapia \& Beck, 2014; Tapia \& Breitmeyer, 2011), which did not rely on local contour interaction, but at a level of object substitution (Di Lollo et al., 2000; Enns, 2004). The perception of a rotational movement was explained with a failed individuation of the target's shape. Whereas the perception of a temporally segregated target was assume to result from the successful escape of the target's surface information from being integrated within the representation of the mask. Altogether the results provide another evidence for different processes underlying both braches of the metacontrast function (Albrecht \& Mattler, 2016; Neumann \& Scharlau, 2007; Reeves, 1982) Altogether the present investigation contribute the conceptualization of 
metacontrast as multidimensional phenomenon (Albrecht \& Mattler, 2016; Fahrenfort et al., 2007; Sackur, 2013). 
5 | Neurophysiological evidence for the multidimensionality of metacontrast masking

\section{Neurophysiological evidence for the multidimensionality of metacontrast masking}

\subsection{Abstract}

Metacontrast masking has been considered as a multidimensional phenomenon, with differences in the perceptions accompanied by different underlying mechanisms. This investigation provided neurophysiological evidence for the multidimensionality assumption of metacontrast, by associating differences in the phenomenology of the target with different neuronal processes. Event-related potentials (ERPs) of conditions with identical physical stimulation, which differed only in the perception of the participants, were compared by cluster-based permutation analyses. Two distinct clusters were found, for the perception of a rotational movement between target and mask and for the perception of a target temporally segregated from the mask. The clusters differed in the spatial and temporal extend and showed an opposite direction of effects. Cluster 1, which describes the signature of the perception of a rotation movement, was associated with later components (P3 or late positivity) which may reflect cognitive revaluations of the perceptual content, whereas Cluster 2 found for the perception of a temporally segregated was associated cognitive demands, for example working memory. Cluster 2 was highly specific for the perception of a segregated target, whereas Cluster 1 was at least partially generalizable to this perception. Evidence for two distinct clusters based on the phenomenology of the target highlights methodological problems for the study of ERPs in metacontrast masking paradigms. Since neuronal processes differ depending on which aspect of the target is considered, statements about neural correlates may be subject to error without considering the appearance of the target. 


\subsection{Introduction}

Most studies examining neural correlates of consciousness (NCC) in masking paradigms contrasted trials with and without awareness of the target. The manipulation of visibility was achieved by the use of masks with varying strengths (Railo \& Koivisto, 2009; van Aalderen-Smeets, Oostenveld, \& Schwarzbach, 2006), the contrast of masking versus nomask conditions (Koivisto, Revonsuo, \& Lehtonen, 2006) or the contrast of different SOAs associated with differences in the target visibility (Koivisto \& Revonsuo, 2008; Pitts, Metzler, \& Hillyard, 2014; Railo \& Koivisto, 2009). These approaches could prove problematic for metacontrast masking, since this paradigm was proposed to be a multidimensional phenomenon, with differences in the perception of the target in dependence of the temporal or spatial relationship between target and mask (Albrecht \& Mattler, 2016; Sackur, 2013). In addition, most studies examining NCC in masking paradigms have not differentiated variations in the phenomenology of the target. Even neural correlates of subjective awareness were measured either by reports whether the target was perceived or not (e.g. Lamy et al., 2008; Pins \& ffytche, 2003; Salti, Bar-Haim, \& Lamy, 2012) or by ratings of the target's visibility (Del Cul et al., 2007; Koivisto \& Grassini, 2016; Sergent, Baillet, \& Dehaene, 2005). These measures captured the perception of the target as whole object, without distinguishing variations in the appearance of the stimulus. Such an approach could underestimate the complexity of target processing. This is particularly problematic for paradigms on which the perception of a stimulus appears to vary across different perceptual dimensions, such as metacontrast masking.

Kanwisher (2001) reviewed neurophysiological evidence that differences in the perception were accompanied by differences in the neural processing, even under identical stimulation. She concluded that perceptual awareness is a multifaceted phenomenon whose neural correlates vary depending on the particular aspects of target being focused on, for example the category or the perceptual attribute of the stimulus. Differences in the neural processing depending on the perceptual awareness were found for motion perception and even mental imagery of motion, which were accompanied by activation of MT/MST (Kanwisher, 2001). Also contour integration, defined as a grouping process of edge or boundary elements to separate an object from surface properties, has been associated in ERP studies with a negative amplitude shift around 150-300 ms after stimulus onset at posterior electrodes, called contour integration negativity (Pitts \& Martínez, 2014). A sensory effect of color was reported 130-170 ms after stimulus onset (Pitts et al., 2014). This exemplary list of different 
perceptions and their electrophysiological correlates is intended to illustrate the importance to consider the phenomenology of an object in order to make statements about its neuronal processing. Statement about NCCs based on the contrast of conditions with different SOAs or masks appears to be problematic as neural processing may reflect qualitative differences in perception rather than differences in mere visibility. In addition, the exclusive consideration of the target processing as a whole object may be insufficient to map the richness of perceptual experience in the form of electrophysiological markers. For metacontrast masking it might be rather appropriate to define the perceptual dimension of the target to identify the neural correlates of their awareness.

Variations in the perception of the target under metacontrast masking were associated with different mechanisms, which depended on the temporal (Neumann \& Scharlau, 2007; Reeves, 1982) or spatial relationship between target and mask (Albrecht \& Mattler, 2016). In Experiment 3 of this thesis it was investigated whether differences in the experience of the target can be attributed to one or two underlying mechanisms. Among others, two perceptions were observed, a Rotation and a Target before Mask. The latter describes the perception of two stimuli separated in time, with the target appearing temporally segregated from the mask. A rotational movement resulted from the interplay of form incongruent target and mask forms. Although both perceptions showed a similar relationship with the temporal parameter, both perceptions were found to be independent of each other in Experiment 3 of this thesis. This was interpreted as evidence for two independent mechanisms underlying both perceptions and confirmed the findings of Albrecht and Mattler (2016). Therefore, the perception of the target under metacontrast masking does not cover the whole object, but is rather limited to different aspects of the target. Even if behavioral evidence was found for differences in the perception of the target in a metacontrast masking paradigm, neurophysiological evidence for distinct mechanisms associated with differences in the perception is still missing.

To provide neurophysiological evidence for the multidimensionality assumption of metacontrast masking, differences in neural processing in dependence of the perception of the target should be observed. Based on the behavioral results, it is expected that both perceptions are elicited by two distinct neural processing mechanisms, which can be distinguished by differences in the temporal and or spatial activity pattern. In addition, if evidence for differences in the neuronal processing in dependence of the perception would be found, the procedure of previous studies using metacontrast masking to search for NCC would be challenged. 
To avoid methodological limitations of previous studies the Hillyard principle (Luck, 2005) was considered in the present investigation and trials with identical physical stimulation were contrasted, which differed only in the perception of the participants. Furthermore, instead of using one-dimensional subjective or objective measures, participants were introduced on which aspect of the target they should focus at. This procedure allowed exploring whether differences in the perception of the target, in detail the perception of a rotational movement or target temporally segregated from the mask, were associated with differences in the neural processing. In addition, it provided electrophysiological evidence for the assumption of different processes underlying metacontrast masking at long SOAs, associated with these perceptions. Since event-related potentials (ERPs) allow a fine temporal resolution, these neurophysiological measures are especially suitable for exploring the time course of the neural target processing. In a first step, the neuronal signature of both perceptions was examined. Therefore, trials on which the respective perception was reported were contrasted with trials on which it was not reported. To evaluate the specificity of both signatures, it was observed whether the pattern of neural activity, found for one perception, yielded also a significant difference between perceived and un-perceived trials of the respective other perception. Finally, for each pattern of neural activity, it was evaluated whether a difference between both perceptions could be found. Since no hypotheses about the exact temporal or spatial differences of the neuronal signatures of both perceptions could be formulated, an explorative analysis was carried out.

\subsection{Methods}

Participants. Sixty-seven (17 male, 50 female) naive students between 19 and 27 years $(M=22.4$ years, $S D=2.1$ years $)$ were recruited. Based on previously defined exclusion criteria, 46 participants were excluded after two behavioral sessions: Twenty-one subjects were excluded because at least one of the two perceptions did not show the expected increasing time course. Fifteen subjects were excluded because they did not show the minimum number of trials for at least one of the three answer alternatives. Eight subjects were excluded because they did not meet both criteria. Twenty-one subjects were admitted for the following EEG sessions. After performing the EEG sessions another 4 participants had to be excluded, since they did not reach the minimum number of 80 trials for at least one of the three answer alternatives in the EEG sessions after artifact exclusion. Therefore, the data from 17 subjects were included in the analysis. The first two behavioral sessions lasted about 90 
minutes, the EEG sessions about 180 minutes. All participants were right-handers, had normal or corrected to normal vision and gave their informed consent. They either received monetary reward or course credits.

Task. The exact task differed between Session 1 and the other Sessions. In Session 1 a two-alternative forced choice task was performed. On half of the blocks participants answered whether they perceived a Flickering or a Rotation and at the other half of the blocks whether they perceived a Target inside Mask or a Target before Mask. A Target inside Mask was defined as the perception of a temporal integration of target and mask, whereas a Target before Mask was described as segregation of both stimuli. Participants were informed that they had to choose between these two perceptions even in trials on which they did not perceive a target. This task served as training to differentiate between these two hard to distinguish perceptions. It should be ensured that in the following sessions, on which only Target before Mask was queried, this perception was affirmed only if a temporal segregation between the two stimuli was perceptible. A Rotation was defined as rotational movement either perceived within the transition of target and mask or a rotational movement of the mask alone, in trials where the target was not visible. A Flickering was defined as the perception of any dynamic sequence attributed to the brief presentation of target and mask, excluding a rotational movement. This comparison was used to ensure that not every dynamic sequence was equated with a rotational experience.

In Session 2-5 participants performed two yes-no tasks on each trial by answering whether they perceived a Rotation and a Target before Mask. A Target before Mask should be negated if either no target was visible or if the target was perceived as being integrated inside the mask. After the first block of Session 2 participants were asked to describe their perceptions, to ensure that the change of the task did not lead to any misunderstandings. At the end of each session a debriefing was done, where subjects were asked to describe and sketch their perceptions.

Stimuli, Procedure and Design. At all sessions, stimuli and trial sequence were identical to the description in General Methods (Figure 2.1), with the following particularities: On each trail subjects gave their responses via mouse click on one of two response fields, which were presented centrally at the bottom of the screen. The cursor was displayed as a small, black cross, displayed on each trial on a random spot at a radius of maximum 350 pixels around the center of the screen. The response fields changed color from light to dark gray when the cursor was placed in their area or after an answer was given. At Session 1-2 the response fields were presented $600 \mathrm{~ms}$ after mask offset. At the EEG-Sessions the response 
keys were displayed $1000 \mathrm{~ms}$ after the mask offset to avoid neural motor response preparation. The assignment of the answer options to the left and right response field was randomized. By giving a response the next trial started automatically. If two answers per trial were requested (Session 2-5) the second response fields occurred automatically after the first answer was given and the next trial started automatically after the second answer. The order of the queried perceptions (Rotation and Target before Mask) varied randomly between trials. This resulted in four different answer alternatives: "Rotation yes - Target yes", "Rotation yes Target no", "Rotation no - Target yes" and "Rotation no - Target no". As listed below, the design varied across sessions, such as the frequency of presentation of the SOAs or the exclusive presentation of incongruent trials in Session 2-5. But none of the subjects noted these differences between sessions as stated by the debriefings at the end of each session.

Training-Session. The first session served as training and was therefore excluded from analysis. It consisted of a slow warm-up block with 8 trials, followed by 10 blocks, 24 trials each. The query of the perceptions alternated blockwise and the order was balanced across participants. At the end of block 2-5 subjects were asked by the experimenter to describe their perceptions and were corrected if necessary.

Screening-Session. Session 2 consisted of a slow warm-up block with 4 trials, followed by 8 blocks, which were separated in two experimental parts, both used to determine one of the two exclusion criteria. The first part consisted of 3 blocks, 60 trials each, independent variables Target (square vs. diamond), Mask (square vs. diamond) were balanced that only incongruent target-mask combinations were presented. The square and diamond forms of the stimuli and $\operatorname{SOA}(36,60,84 \mathrm{~ms})$ varied pseudo-randomly within each block so that each of the 6 combinations occurred equally often. A pause was offered after every 30 trials. The increase in the frequency of both perceptions with SOA was used to determine the first exclusion criterion. Subjects who showed no increase in absolute frequencies between the first and second SOA or the first and third SOA for either of the two perceptions were excluded. This criterion was based on the results of the first three experiments which provided an increasing time course for both perceptions.

The second part of Session 2 consisted of 5 blocks 48 trials each. The $60 \mathrm{~ms} \mathrm{SOA}$ was presented 40 trials per block, 200 times in total. The other two SOAs (36, $84 \mathrm{~ms})$ were presented randomly intermixed within each block for 4 trials each, 20 times each in total. Only incongruent target-mask combinations were shown. The square or diamond form of the stimuli and SOA varied pseudo-randomly within each block so that each combinations occurred equally often within both, the $60 \mathrm{~ms}$ SOA and the other two SOAs. On each trial, 
two answer fields were displayed consecutively on the screen with which the subjects gave for each perception their yes-no answers. For the analysis of the EEG data, only those trials were of relevance on which the subjects had either affirmed only one of the two perceptions or had denied both perceptions. Therefore, the second exclusion criteria defined whether participants reported each of three following answer options, "Rotation yes - Target no", "Rotation no - Target yes" and "Rotation no - Target no", in minimum 10\% $(N=20)$ of the trials at $60 \mathrm{~ms} \mathrm{SOA}$. If the minimum frequency required for at least one of the three response alternatives was not met, the subject was excluded.

EEG-Sessions (3-5). The stimuli, procedure and design of the EEG-Sessions were identical to the second part of Session 2, except 10 blocks, 44 trials each were presented at each EEG-Session. The $60 \mathrm{~ms}$ SOA was presented 32 trials per block, 320 trials per session. The 36 and 84 ms SOA were presented randomly intermixed within each block for 6 trials each, 60 trials per session each.

EEG Recording and Processing. EEG was acquired via the BioSemi ActiveTwo recording system (BioSemi Inc. Amsterdam, The Netherlands) with $64 \mathrm{Ag} / \mathrm{AgCl}$ electrodes arranged on a standard BioSemi head cap according to the International 10-20 system and a sampling rate of $512 \mathrm{~Hz}$. To record horizontal eye-movements electrooculogram (EOG) electrodes were applied to the external canthi. Vertical eye-movements and blinks were recorded by a monopolar electrode placed below the left eye and the average between the two frontal EEG electrodes FP1 and FP2. The mastoid electrodes were used as references. The MATLAB toolbox EEGLAB (Delorme \& Makeig, 2004) was used for signal processing.

The data was binned in a time window of $-200 \mathrm{~ms}$ to $900 \mathrm{~ms}$, reference to the target onset. A band-pass filter between $0.5-30 \mathrm{~Hz}$ was applied. An independent component analysis (ICA) was performed to correct for eye artefacts, with noisy electrodes and trials with strong artifacts excluded from ICA. Afterwards, trials with any of the following artefacts within the epoch of -200 -900 ms were excluded from further analysis: activity $\pm 70 \mu \mathrm{V}$ on the vertical or horizontal EOG electrodes, activity $\pm 100 \mu \mathrm{V}$ of the 64 EEG electrodes or an exceedance of the sample-to-sample voltage threshold of $50 \mu \mathrm{V}$. From ICA excluded electrodes were interpolated afterwards and a baseline correction from -200 ms to target onset was applied. EEG recording took place in a separate recording room, which served as a Faraday cage. Participants sat in an armchair without chin rest, with the experimental monitor outside the recording room, visible through a specially treated shielded glass pane. They were instructed to avoid eye movements or blinks and to fixate the fixation cross until the response keys were displayed on the screen. 
Statistical Analysis of Event-related Potentials. To study the neural signatures of the perceptions (1) Rotation and (2) Target before Mask, trials were divided into conditions depending on the subject's response. To observe the neural signature of Rotation, eventrelated potentials (ERPs) of trials on which participants reported to perceive "Rotation yes Target no" were contrasted with ERPs of trials on which "Rotation no - Target no" was reported. To observe the neural signature of Target before Mask, ERPs of trials on which "Rotation no - Target yes" was reported were contrasted with trials on which "Rotation no Target no" was reported.

For both comparisons, nonparametric cluster-based permutation analyses (Maris \& Oostenveld, 2007) were performed. This procedure allows the analysis of ERPs at any time point and within any electrode, while controlling for family-wise error. First, paired t-tests were performed for each time point and electrode. Second, two-dimensional clusters based on temporal and spatial adjacency of t-test above or below the predefined threshold of $t= \pm 2.1$ were built, whereas clustering was performed separately for positive and negative t-values. Third, for each cluster, the sum of all t-statistics was calculated, the so-called observed cluster-level statistic. Fourth, 10.000 permutations were performed with answers randomly assigned to trials. To determine the $p$-value of each cluster, the proportion of permutations was calculated with a cluster-level statistic exceeding the observed cluster-level statistic. Since all significant clusters $(p<.025)$ were considered, the permutation distribution of the largest cluster had to be taken into account for calculating the $p$-value in order to control for the family-wise error.

To anticipate the results in advance, two significant clusters were found. Cluster 1 describes differences in activity between trials on which "Rotation yes - Target no" was perceived compared to trials on which "Rotation no - Target no" was perceived. Therefore, Cluster 1 represents the neural signature of the perception of a Rotation. Cluster 2 describes differences in the activity between trials on which "Rotation no - Target yes" was perceived compared to trials on which "Rotation no - Target no" was perceived. Therefore, Cluster 2 represents the neural signature of the perception of a Target before Mask.

In order to consider the variation of the clusters over time, both clusters were subdivided into five time ranges, defined by the particular constellation of electrodes which were part of the cluster at the given time range. The subdivision was made via visual inspection on the basis of the electrode-time matrix, which showed for each cell of the cluster the strength of activity differences of grand averaged ERPs. The subdivision was used to 
visualize the temporal development of the clusters in the form of grand averaged ERPs or as topographies.

Subsequently, the specificity of both clusters was tested in two steps. For this purpose, further cluster-based permutation analyses were carried out with 10.000 permutations per analysis. Since only one-sided subsequent analyses were calculated, the critical alpha-level was set to .05 . On the one hand, the generalizability of one cluster to the respective other percept was analyzed. It was examined whether Cluster 1, representing the perception of a Rotation, resulted in a significant effect for the perception of a Target before Mask. Therefore, trials on which "Rotation no - Target yes" was perceived were compared to trials on which "Rotation no - Target no" was perceived. For Cluster 2 it was examined whether a significant effect for the perception of a Rotation could be found. The activity of trials on which "Rotation yes - Target no" was perceived were contrasted with trials on which "Rotation no Target no" was perceived. On the other hand, it was examined whether the contrast of both perceptions yielded significant effect for both clusters. For Cluster 1, the activity of trials on which "Rotation yes - Target no" was reported was contrasted with trials on which "Rotation no - Target yes" was reported. For Cluster 2 the activity of trials on which "Rotation no Target yes" was perceived was contrasted with trials on which "Rotation yes - Target no" was perceived.

\subsection{Results}

\subsubsection{Behavioral data}

For repeated measures ANOVA Huyn-Feldt corrected $p$-values were reported, but to facilitate readability uncorrected degrees of freedom were reported. An arcsine square root transformation was applied to relative frequencies before analysis. The frequencies of reports of a Rotation and a Target before Mask showed an increase across SOA at the Screening Session (Figure 5.1A). A two-factorial repeated measures ANOVA yielded a significant effect of SOA $(F(2,32)=73.37, p<.001)$, a significant effect of perception $(F(1,16)=9.87$, $p=.006)$ and a significant interaction $\operatorname{effect}(F(2,32)=12.3, p<.001)$.

The same pattern was found at the EEG-Sessions, with both perceptions yielding an increase across SOA (Figure 5.1B) and a two-factorial repeated measures ANOVA yielding a significant effect of $\operatorname{SOA}(F(2,32)=65.67, p<.001)$, a significant effect of perception $(F(1,16)=8.78, p=.009)$ and a significant interaction effect $(F(2,32)=12.59, p=.001)$. 
A

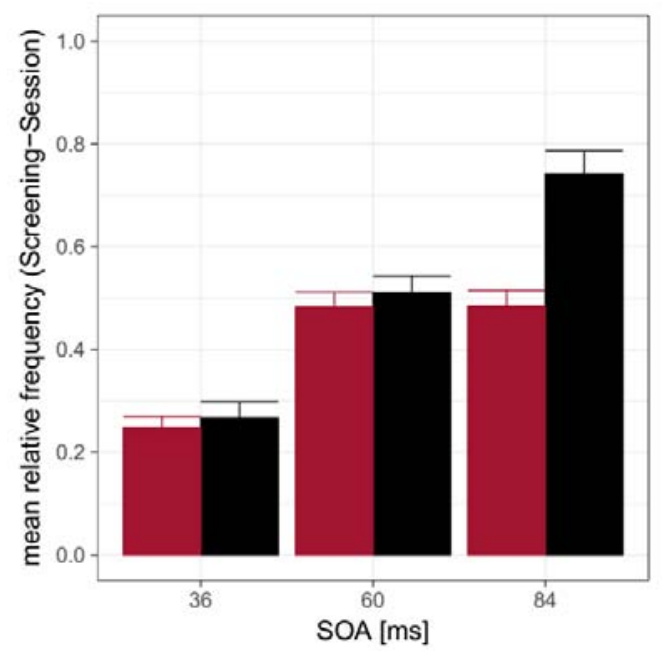

B

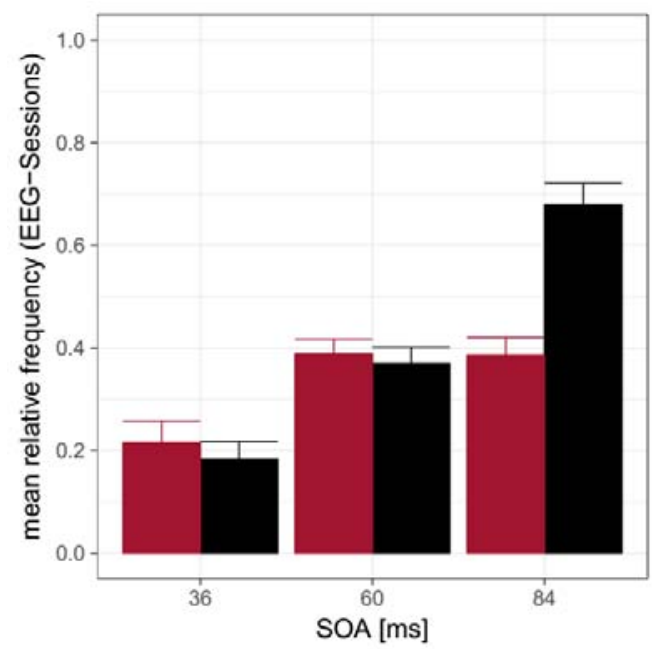

Figure 5.1. Mean relative frequency of Rotation (red) and Target before Mask (back) for participants which were included in the analysis after the EEG-Sessions $(N=17)$. Error-bars depict betweensubject standard errors of the mean. A Mean relative frequency for both perceptions at the ScreeningSession. B Mean relative frequency for both perceptions at the EEG-Session.

\subsubsection{Event-related potentials}

Neural signature of Rotation. A cluster-based permutation analysis for the comparison of activity of trials on which "Rotation yes - Target no" was reported in contrast to trials on which "Rotation no - Target no" was reported, yielded a significant positive cluster (Cluster 1 , test statistic $=7086, p<.001)$.

Cluster 1 covered the time range $230-553 \mathrm{~ms}$ after target onset. The spatial and temporal extent as well as the subdivision of Cluster 1 in five time ranges is depicted in an electrode-time matrix of Figure D1A (Appendix IV). This matrix visualized the activity differences of the two perceptions for the cells which were part of Cluster 1 . The visualization of the results of Cluster 1 by ERPs and topographies (Figure 5.2) was separated into the five time ranges presented in the electrode-time matrix of Figure D1A (Appendix IV).

ERPs were averaged across electrodes defining Cluster 1 in the respective time range (Figure 5.2A). The amplitude of ERPs elicited by the perception of "Rotation yes - Target no" were higher compared to the amplitude of ERPs elicited by the perception of "Rotation no - Target no". Topographies visualized differences between EPRs elicited by the two perceptions, "Rotation yes - Target no" and "Rotation no - Target no", averaged across the time range of the particular subdivision (Figure 5.2B). The first subdivision of Cluster 1 covered the time range $230-295 \mathrm{~ms}$ and showed a large spatial distribution. The second time range $(296-365 \mathrm{~ms})$ depicted activity differences at fronto-parietal electrodes. The third time 
range $(366-438 \mathrm{~ms})$ showed a lateralization on the right hemisphere in the area of frontoparietal electrodes. A large spatial distribution was found at $439-516 \mathrm{~ms}$, whereas activity differences of the fifth time range $(517-553 \mathrm{~ms})$ were limited to occipital-temporal electrodes over the left hemisphere.

Specificity of the neural signature of Rotation. In a second step a subsequent cluster-based permutation analysis examined whether Cluster 1 resulted also in a significant effect for the perception of a Target before Mask. The analysis yielded a significant effect (test statistic $=3672, p=.005$ ). The amplitude of ERPs elicited by trials on which "Rotation no - Target yes" was reported exceeded the amplitude of ERPs elicited by trials on which "Rotation no - Target no" was reported (Figure 5.2A). However, the activity difference between these two answers was smaller, compared to the original contrast of Cluster 1 between the answers "Rotation yes - Target no" and "Rotation no - Target no", as also reflected in the topography maps (Figure 5.2C), especially in time ranges three, four and five. In addition, an inverse effect was found with stronger activity for trials on which "Rotation no - Target no" was reported compared to trials on which "Rotation no - Target yes" was reported, mainly in the first time range $(230-295 \mathrm{~ms})$ at occipital electrodes and in the last time range $(517-553 \mathrm{~ms})$ at occipital-temporal electrodes.

The third analysis examined whether a difference between the perception of a Rotation and the perception of a Target before Mask could be found for Cluster 1. A cluster-based permutation analysis was performed by contrasting trials on which "Rotation yes - Target no" was reported with trials on which "Rotation no - Target yes" was reported. The analysis yielded a significant effect (test statistic $=2628, p=.035$ ). ERPs (Figure 5.2A) and topography maps (Figure 5.2D) showed a stronger activity for trials on which "Rotation yes Target no" was perceived compared to trials on which "Rotation no - Target yes" was perceived. The strongest activity difference was visible at the latest time range (517$553 \mathrm{~ms})$. 

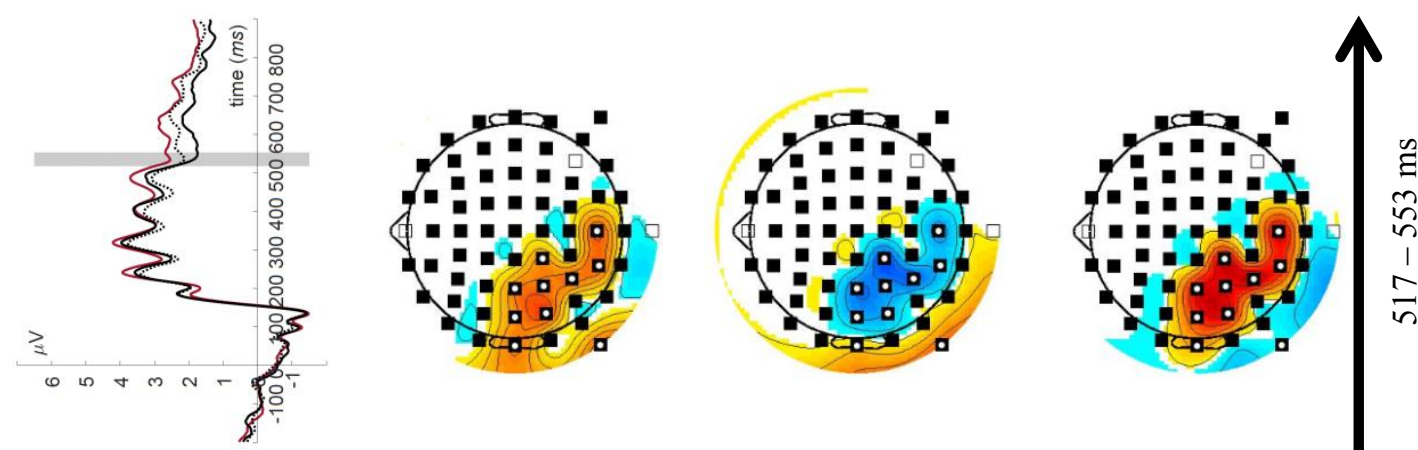

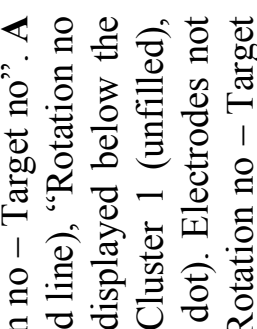

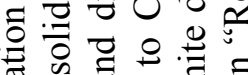

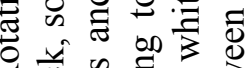

듀

르응
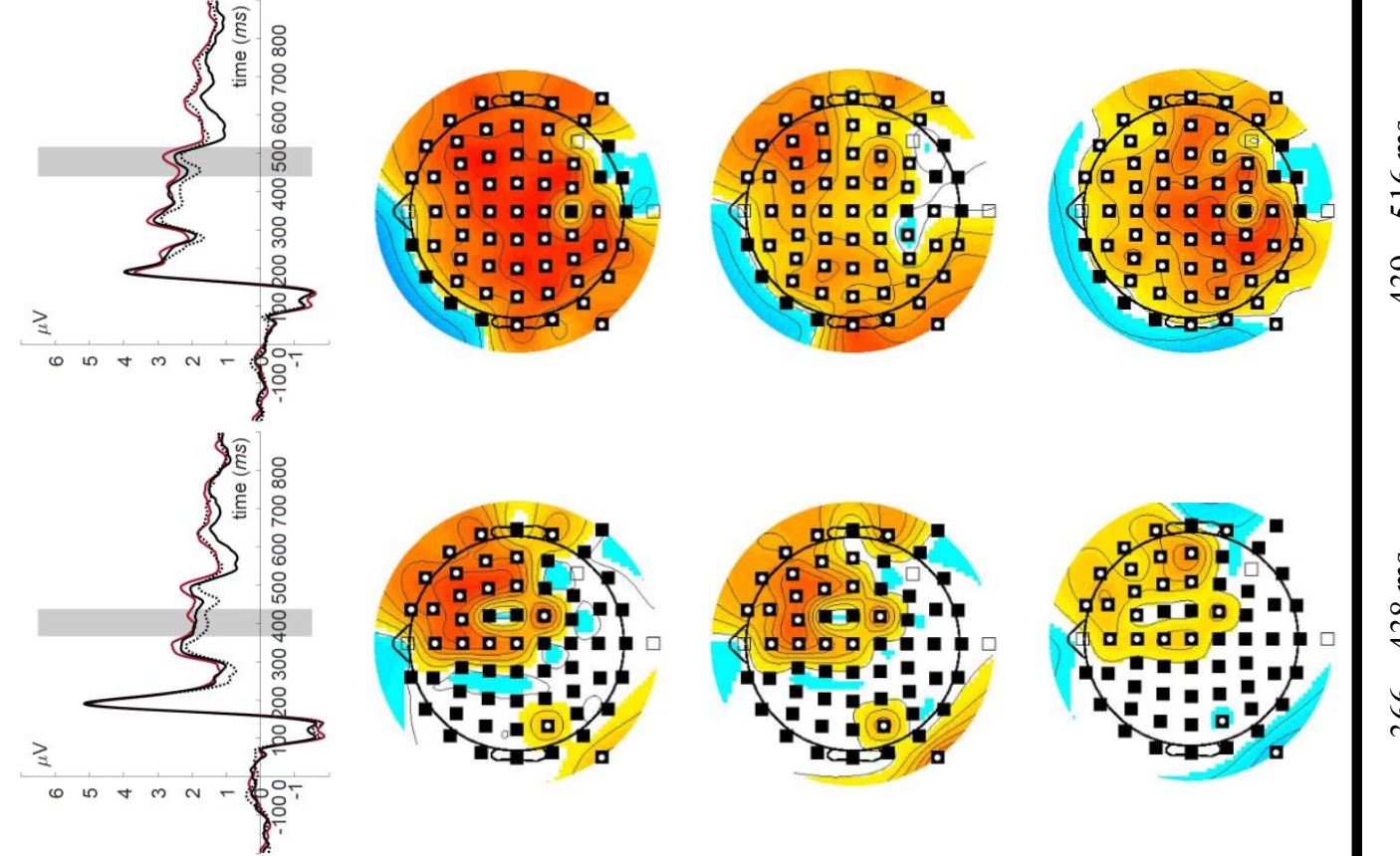

is

空各兑惫音

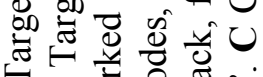

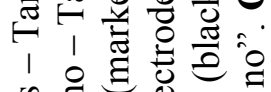

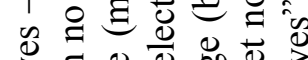

¿ 0000

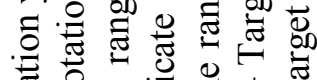

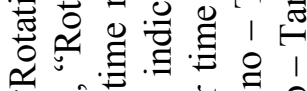

के

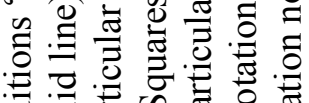

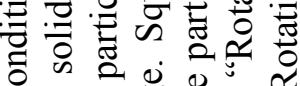

ठृ की

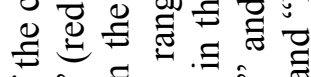

फั0

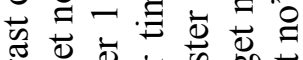

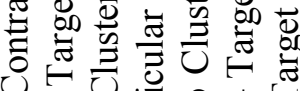
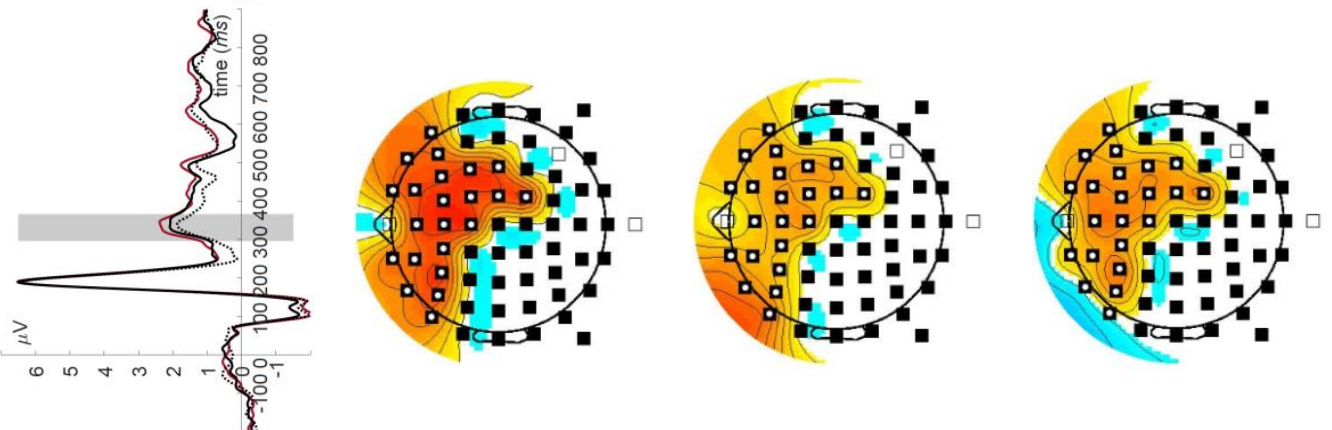

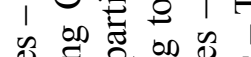

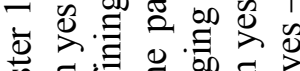

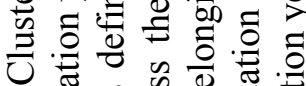

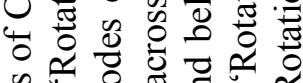

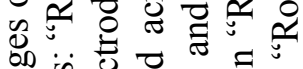

范

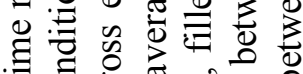

0 0 क

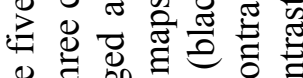

\& $\exists$ o

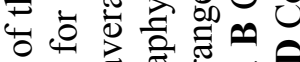
क के एं

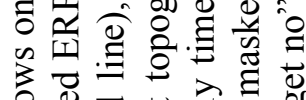
क

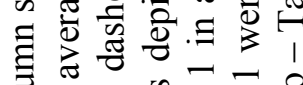

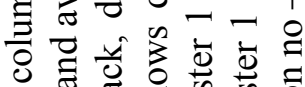
㐘 II ठ⿹𠃌 ㄱํㄴㅇㅝ

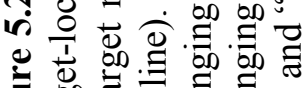

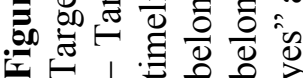



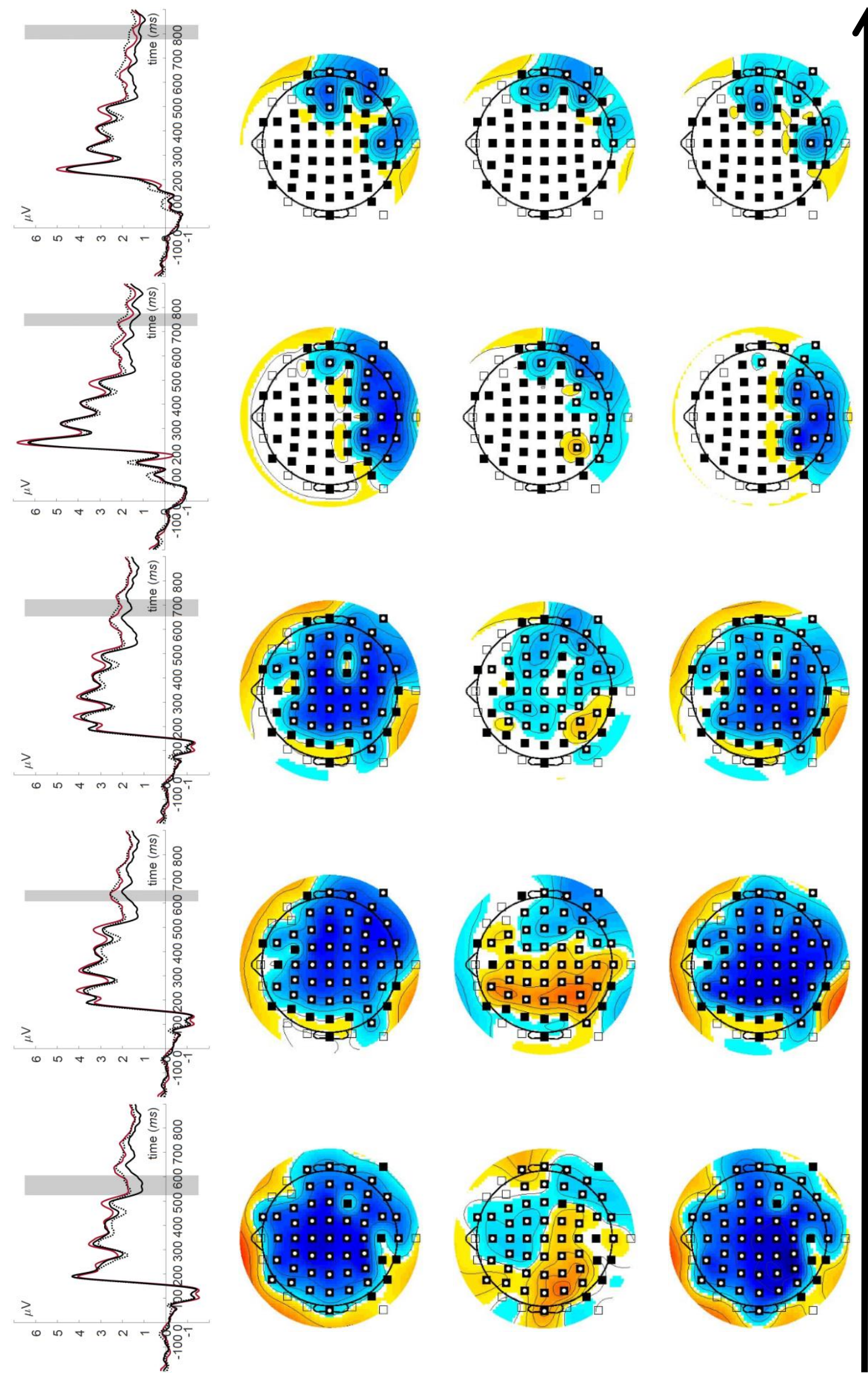

욛요

需离离

为完

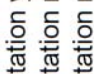

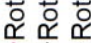
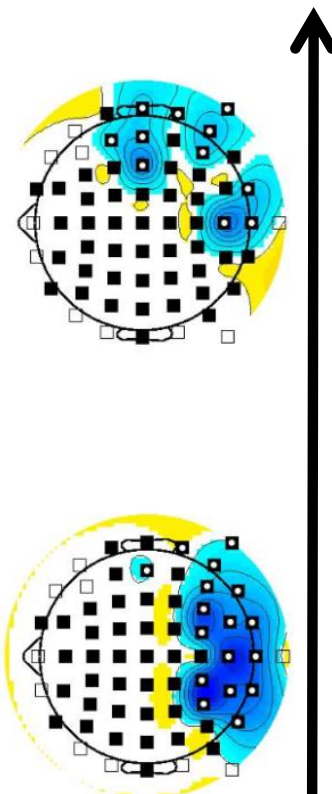

1

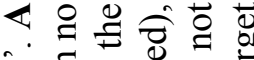

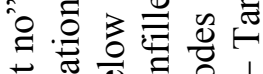

峁

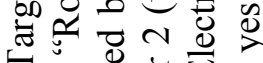

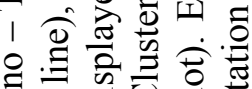

च.

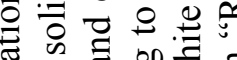

के

중

를으용

的死 8

is 2000

范各要

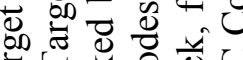

สิ

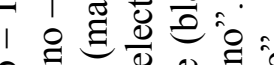

马 00 o

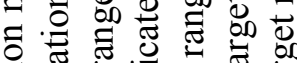

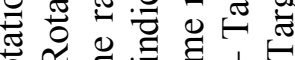

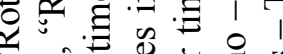

बें

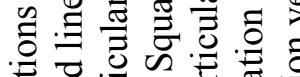

光 : :

त :

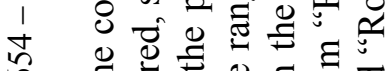

$\nsubseteq \underset{\Xi}{\Xi} \Xi \Xi$

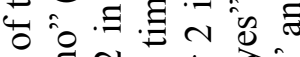

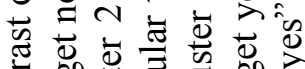

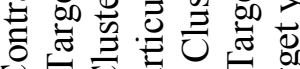

ن

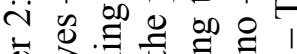

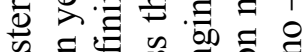

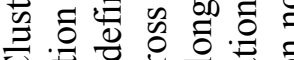

ర

फे

论

त्ञ

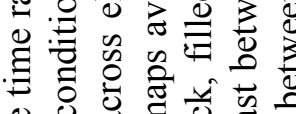

¿ 0 ర

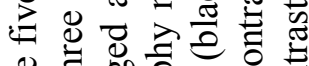

纯

पे

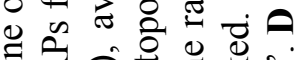

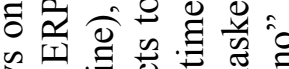

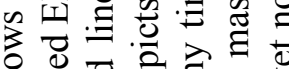

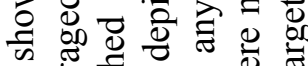

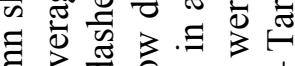

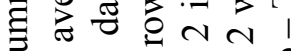

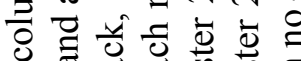

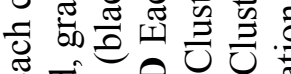

Iี

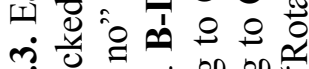

ले :

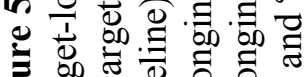

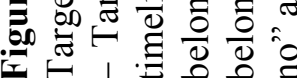


Neural signature of Target before Mask. A cluster-based permutation analysis, comparing the activity of trials on which "Rotation no - Target yes" was reported with trials on which "Rotation no - Target no" was reported, yielded a significant negative cluster (Cluster 2, test statistic $=-6528, p<.001)$. Cluster 2 covered the time range between $525-$ $836 \mathrm{~ms}$ after target onset, as visualized in the electrode-time matrix (Figure D1B, Appendix IV). ERPs elicited by trials on which participants reported to perceive "Rotation no - Target yes" showed more negative amplitudes than ERPs elicited by trials on which "Rotation no Target no" was reported (Figure 5.3A). Therefore, Cluster 2 which described the neural signature of a Target before Mask yielded less activity in conditions on which a Target before Mask was perceived compared to conditions on which a Target before Mask was not perceived. Accordingly, the topographies showed a negative activity differences (Figure 5.3B). The first $(525-606 \mathrm{~ms})$, second $(607-653 \mathrm{~ms})$ and third time range $(654-723 \mathrm{~ms})$ of Cluster 2 showed a large spatial distribution, with only slight differences in the respective topographies. The activity in the fourth time range $(724-776 \mathrm{~ms})$ was limited to occipital electrodes, similar to the fifth time range $(777-836 \mathrm{~ms})$ which showed activity differences at occipital-temporal electrodes over the right hemisphere.

Specificity of the neural signature of Target before Mask. The second subsequent cluster-based permutation analysis examined whether Cluster 2, found for the perception of a Target before Mask, also yielded a significant effect for the perception of a Rotation. This analysis did not yield a significant effect (test statistic $=-769, p=.30$ ), indicating that trials on which a Rotation was not perceived did not yield a significant lower activity compared to trials on which a Rotation was perceived. Descriptive, a difference between ERPs of the conditions "Rotation yes - Target no" and "Rotation no - Target no" were found in the last time range (777 - $836 \mathrm{~ms}$ ) (Figure 5.3A). The topography maps (Figure 5.3C) showed for the first two time ranges slightly positive activity differences over the left hemisphere as well as slightly negative activity differences over the right hemisphere. Also the third (654-723 ms) and fourth time range $(724-776 \mathrm{~ms})$ showed an unsystematic pattern of activity differences, with some electrodes showing slightly positive and others showing slightly negative activity differences.

The third subsequent cluster-based permutation analysis examined whether significant activity differences between the perception of a Rotation and a Target before Mask could be found for Cluster 2. A significant difference of activity was found between trials on which "Rotation no - Target yes" was perceived compared to trials on which "Rotation yes - Target no" was perceived (test statistic $=-5470, p=.004$ ). ERPs elicited by the perception of a Target 
before Mask showed more negative amplitudes than ERPs elicited by the perception of a Rotation (Figure 5.3A). In accordance, topography maps depicted activity differences between those two perceptions, which decreased with increasing time range (Figure 5.3D).

\subsection{Discussion}

\subsubsection{Evidence for two distinct neural signatures}

Since this investigation is based on subjective data, interindividual variations in the perception must be anticipated, as well as the problem that there is no objectives criterion to distinguish between correct and wrong answers. Previous investigations (Experiment 1-3 of this thesis) showed an increasing time course for the perceptions of a Rotation and a Target before Mask. This makes it possible determine the accuracy of perceptions of the present investigation. Therefore, the exclusion criteria based on the time course of both perceptions controlled that participants whose perception deviated from the norm were excluded. The conservative exclusion criterion ensured that a possible null effect, for example no difference in neural processing between both percepts, cannot be attributed to interindividual differences in the perception. The high dropout rate can be explained with the difficulty of the task. On the one hand, only a limited range of SOAs was provided. On the other hand, only incongruent trials were presented, which affected mostly the perception of a Rotation. Both factors limited the context of perceptual experience, which made it even harder to differentiate subtle variations in the target experience. Subjects who were admitted to the EEG-Sessions also showed increasing courses for both perceptions in these sessions. The stability of the time courses is surprising regarding the variation of trials per SOA at the EEGSessions. Even if considerably more trials were presented at $60 \mathrm{~ms}$ SOA than at 36 and $84 \mathrm{~ms}$ SOAs, a stability of perception with respect to the temporal parameter was found. Nevertheless, the high dropout rate is a limitation of this investigation. Future research is needed to determine whether the results can be replicated using a more liberal exclusion criterion, without excluding participants who have shown the wrong time course.

Despite the similarity in the behavioral responses regarding the time course and frequencies of reports for both perceptions, neurophysiological differences in the processing in dependence on the perceptions were found. First, the cluster-based permutation analyses yielded for both perceptions a distinct cluster that differed in its temporal extent. Cluster 1 represented the neural signature of the perception of a Rotation and was found at an earlier 
but overlapping time window than Cluster 2, representing the neural signature of the perception of a Target before Mask. Second, the spatial distribution diverged between both clusters. Both clusters showed large spatial distributions in early time ranges and occipital sources at the latest time range. But only for the perception of a Rotation frontal-parietal activity was found at intermediate time ranges. Thus, the spatial pattern of Cluster 2 showed a higher stability compare to Cluster 1. Third, the opposite direction of effects was found for both clusters. For Cluster 1, trials on which a Rotation was perceived yielded larger amplitudes of ERPs compared to trials on which a Rotation was not perceived. In contrast for Cluster 2, the activity elicited by trials on which a Target before Mask was perceived showed smaller amplitudes compared to trials on which a target was not perceived. This also shows that larger ERP amplitudes did not just reflect the perception of any aspect of the target. Instead, only the perception of a Rotation seemed to be accompanied by a larger positivity for trials reflecting the awareness of this perception.

\subsubsection{Specificity of the neuronal signatures}

In a second and third step the specificity of the cluster for the particular perception was analyzed. Cluster 1 seemed to be generalizable to the perception of a Target before Mask. Interestingly, even if the size of the effect was smaller for the perception of a Target before Mask, the direction of effects was the same in the first four time ranges of Cluster 1 . The amplitudes of ERPs were higher in trials on which a target was perceived, compared to trials on which a target was not perceived. But in the last time range, where both clusters overlapped, a reverse direction of effects was found, with smaller amplitudes for trials on which a target was perceived. This shows that the stronger negativity found for Cluster 2 on trials which indicated no awareness of the target, was not specific for the perception of a Target before Mask, but only for this perception at the late time window. Even if Cluster 1 could be generalized to the perception of a Target before Mask, a significant difference between both perceptions was found, with a larger positivity for the perception of a Rotation compared to the perception of a Target before Mask. Therefore, even if this pattern of activity, which differentiates between perceived and not perceived trials, could be found for both target aspects, it still differentiates between the perception of a Rotation and a Target before Mask.

In contrast, Cluster 2 seems to be highly specific for the perception of a Target before Mask. First, no significant difference in the activity was found between trials on which a 
Rotation was or was not perceived. To examine whether the activity elicited by trials on which a Rotation was perceived was lower compare to trials on which Rotation was not perceived, only a one-sided tested was calculated. However, the direction of effects was to divergent between individual electrodes to allow an effect in the other direction. Therefore, it can be concluded that for Cluster 2 no difference in activity could be found regarding the perception of a Rotation.

Second, a significant difference between the perception of a Target before Mask and a Rotation was found. Trials on which a Rotation was perceived showed larger amplitudes of ERPs compared to trials on which a Target before Mask was perceived. Descriptively, however, this difference in amplitudes decreased with increasing time range.

\subsubsection{Implications}

For both perceptions, a Target before Mask and a Rotation, two neural signatures were found which differed in spatial as well as temporal expansion and showed an opposite direction of effects. In addition, Cluster 1 was only partially generalizable to the perception of a Target before Mask and Cluster 2 even showed a high specificity. Thus, these results coincide with the results of Experiment 3 of this thesis which provided behavioral evidence for the independence of the two perceptions. Moreover, the results expand the consideration of metacontrast as a multidimensional phenomenon based on behavioral results (Albrecht \& Mattler, 2016; Breitmeyer et al., 2006; Sackur, 2013), as they offered neurophysiological evidence for the multidimensional assumption of metacontrast masking. This assumption is supported by evidence for distinct neural processes associated with differences in the phenomenology.

This implies that the search for a unitary neural correlate of target visibility could be biased for metacontrast masking regarding differences in the phenomenology. This criticism also applies to the contrast of identical conditions, since differences in the neural processing were found to depend on the target appearance, although physical stimulation and task were identical on each trial. These methodological problems are discussed in detail below.

Experiment 1 and 2 of this thesis provided evidence that the frequency of the perception of different target aspects varies with the temporal or spatial relationship of target and mask. It has been shown, that the target perception differs qualitatively between these conditions on several perceptual dimensions, like contrast, temporal separation or movement. Based on the results of the present investigation it can be assumed, that the neural target 
processing differs between conditions which are accompanied by differences in the phenomenology. Therefore, it could be rather problematic to contrast different conditions in a metacontrast masking paradigm, since phenomenological variations are reflected in the neural processing. Consequently, metacontrast masking studies examined NCCs by the usage of different masks or SOAs (e.g. Koivisto et al., 2006; Railo \& Koivisto, 2009; van AalderenSmeets et al., 2006), may not examining NCCs but rather differences in the neural processing based on difference in the phenomenology.

Some studies controlled the contrast of conditions with different physical stimulation, for example masks, by subtracting ERPs of the mask-only condition from ERPs elicited by the presentation of target and mask (Del Cul et al., 2007; Railo \& Koivisto, 2009). This approach is based on the assumption that there is only an additive effect between target and mask, which can be canceled out by the subtraction. This assumption can be criticized (Bachmann, 2009a). If the target processing would be affected only additively by the mask, qualitative differences in the perception of the target under metacontrast masking could hardly be explained. Mainly perceptions indicating an interplay between target and mask, for example the perception of a rotational movement, illustrate that the target-mask combination results in a qualitatively different percept compared to the perception of the target alone. Since the results of the present investigation provided evidence that differences in the perception are accompanied by distinct neuronal signatures, the subtraction of mask-only ERPs would probably not eliminate an additive effect of the mask processing. Instead, qualitatively distinct neural patterns with differences in the spatial and temporal extend could be subtracted by these method.

The same criticism applies to the contrast of different target-mask combinations. Rutiku, Martin, Bachmann, and Aru (2015) proposed to subtract ERPs from control conditions with those from the experimental condition, to control for differences in the physical stimulation. Again, this claim is based on an additivity assumption, which is untenable in terms of the contrast of different target-mask combinations, since the spatial relation between target and mask seems to affect the target processing qualitatively and not only in a quantitative and thus additive manner. First, Weisstein and Growney (1969) provided evidence that two different perception, the perception of an apparent motion and the perception of a phenomenal absent target, were affected differently by changes in the visual angle between target and mask. With increasing visual angle the metacontrast suppression was reduced, whereas the apparent motion perception was not influenced. Second, the perception of a rotational movement strongly depends on the congruency of both stimuli, 
which has also been shown in Experiment 1-3 of this thesis and previous studies (Ansorge et al., 2007; Ansorge et al., 2009; Maksimov et al., 2011). Third, Albrecht and Mattler (2016) even provided evidence that the spatial layout of the stimuli affects the phenomenology of the target and with it which mechanism underlying metacontrast may emerge. This shows that even subtle variation in the spatial layout of target and mask, may affect the phenomenological outcome tremendously. Consequently, in the case of metacontrast masking a subtraction method seems to be not appropriate, since the assumption of an additive effect in the neural processing of target and mask is not tenable, regarding differences in the phenomenology and the neural processing between different conditions.

Evidence for differences in the neural processing based on the perception of the target, challenges the usage of one-dimensional subjective or objective measures. Interindividual variability in the perception of different target aspects was found (Albrecht et al., 2010; Albrecht \& Mattler, 2012b, 2012a, 2016). Consequently, it is possible that the missing specification of one-dimensional measures, which target aspects should be considered, also results in interindividual differences in the neural processing of the target.

\subsubsection{A cautious interpretation of the functional significance of both neural signatures}

It is an open question how to interpret the neuronal signatures indexing awareness of both perceptions. Since an exploratory framework has been used, content interpretations of the clusters have to be treated with caution. Nevertheless, the late latency found for both clusters indicates that differences in the perception do not emerge within the early, bottom-up driven perceptual processing stage which is assume to be completed within $\sim 100 \mathrm{~ms}$ after stimulus onset (for review see Tapia \& Beck, 2014) and is associated with earlier components like C1 and P1 (Di Russo, Aprile, Spitoni, \& Spinelli, 2008). Instead, both clusters seem to reflect rather later processing stages, already influenced by top-down or recurrent feedbackloops.

The temporal and spatial dynamic of Cluster 1 shows similarities with two later components the late positivity (LP) or the P3, respectively, which resembles in time course and topography (Koivisto et al., 2006; Koivisto \& Revonsuo, 2010). These late components emerge around 300-600 ms after stimulus onset with a higher amplitude for trials which indicated awareness of the stimulus and a large spatial distribution with a maxima at the fronto-parieto network (Del Cul et al., 2007; Koivisto \& Revonsuo, 2010; Lamy et al., 2008; Sergent et al., 2005). These criteria match with the neural activity pattern found for Cluster 1 , 
which emerged $\sim 230-550 \mathrm{~ms}$ after target onset with a large spatial distribution across the entire network and a focus on frontal-parietal areas. In addition, larger amplitudes were found for trials on which a Rotation was perceived compare to trials on which it was not perceived. Therefore, it is likely that Cluster 1 corresponds to late components, like P3 and LP.

Late components have not only been termed as NCC (Del Cul et al., 2007), but were also equated with reflexive consciousness or a consequence of conscious perception, like an update in working memory necessary for report (Koivisto \& Revonsuo, 2008; Railo \& Koivisto, 2009; Rutiku et al., 2015; Wilenius \& Revonsuo, 2007). Late components, like P3, were associated with a more central level of information processing, necessary to identify the stimulus (for review see Kok, 1997, 2001). Koks reviews summarized evidence that the P3 is sensitive to attentional or working memory mechanisms at categorization tasks. In accordance, the task of the present investigation requires the maintenance of the perception in working memory, necessary for the comparison with an internal set of representation.

The P3 was assumed to reflect the confidence of a decision in a change detection task (Eimer \& Mazza, 2005). Also for the present investigation amplitude differences may be traced back to differences in confidence regarding the perceptual decision. Participants may feel more confident in trials on which they were able to identify one of the perceptions, compare to trails on which they negate to perceive anything. Lamy et al. (2008) criticized the interpretation of Eimer and Mazza (2005), since the P3 amplitude differed also in terms of subjective awareness, but only under a constant high confidence level, whereas the amplitude did not show variations with subjective awareness under low confidence level. Even if an effect of the confidence on Cluster 1 cannot rule out, the interaction found by Eimer and Mazza (2005) indicates that the confidence level has a moderating rather than a deterministic effect on the P3 amplitude.

Based on the assumption that Cluster 1 is associated with a later component, as P3 or LP, this neural signature seems to reflect not a mere bottom-up perceptual processing, but rather an interaction between perceptual information and higher cognitive processes, like working memory, which helps to evaluate or categorize the visual information. Furthermore as Kanwisher (2001) highlighted, the conscious perception of a perceptual content is not only maintained by its neural representation, but also interactions between this neural representation with other cognitive processes are needed for conscious awareness.

Even if Cluster 1 represents the perception of a Rotation, also for the perception of a Target before Mask significant differences between seen and unseen trials were found. This implies that also for the perception of a temporally segregated target, the first distinction 
between perceived and un-perceived trials could be found on a stage of cognitive evaluation of visual information. Nevertheless, a significant difference between the perception of a Rotation and the perception of a Target before Mask was found for Cluster 1, indicating a partial generalizability of Cluster 1. This fits with the result that late ERP components (LP) differed depending on whether local or global shapes of a stimulus was to be considered (Koivisto et al., 2006). Thus, late components may be modulated by the stimulus aspects, participants are focusing at.

The neuronal signature for the perception of a Target before Mask shows larger amplitudes for trials on which a target was not perceived compare to trials on which it was perceived. This contrasts with the direction of effects found for late components (P3 or LP), which showed larger amplitudes for trials indicating awareness. In addition, the late latency of Cluster $2(\sim 530-840 \mathrm{~ms})$ makes it unlikely to interpret it as P3 or LP. Due to this latency it is unlikely that Cluster 2 represents a mere perceptual process. Instead, the late latency of Cluster 2 is comparable with a slow negativity wave which has been interpreted to index central resources, like visual short-term memory process (Mecklinger \& Pfeifer, 1996; Ruchkin, Canoune, Johnson, \& Ritter, 1995; Vogel \& Machizawa, 2004).

The amplitude of the negativity wave was found to be affected by the number of items which have to held in memory (Vogel \& Machizawa, 2004). The author interpreted this result as evidence that the negativity wave rather reflect memory capacity, instead of executive processes. An association of Cluster 2 with the negativity wave implies that the perception of a Target before Mask requires more visual short-term memory compare to the perception of a Rotation, since Cluster 2 was found to be specific for the perception of a Target before Mask, with lower amplitudes for this perception compared to the perception of a Rotation. This could be explained by the need to consider the perception of two stimuli, target and mask as well as their temporal relation, before deciding whether a Target before Mask has been perceived or not. In contrast, for the perception of a Rotation only the movement itself must be perceived which may require lower memory demands. But this post-hoc explanation should be required with cautions. Instead, further investigations are needed to observe differences in the memory demand regarding both perceptions. Since stimuli were always presented centrally in the present investigation, lateralization effects used for the investigation of slow negativity wave (Klaver, Talsma, Wijers, Heinze, \& Mulder, 1999; Vogel \& Machizawa, 2004) were not visible in the neural pattern of Cluster 2. In addition, the focus on occipital or parietal electrodes of the slow negativity wave (Klaver et al., 1999; Vogel \& Machizawa, 2004) differed from the large spatial distribution of Cluster 2. Therefore, based 
on present results it cannot be stated with certainty that Cluster 2 reflects a late negativity wave, indicating visual short-term memory process. Nevertheless, the late latency of Cluster 2 rather excludes perceptual processes and makes it more likely that this neural signature reflects any kind of central resources, as working memory (Kok, 1997).

\subsubsection{Limitations}

First differences between perceived and un-perceived trials were found at rather late components, which were interpreted to reflect cognitive evaluations of perceptual information (Cluster 1) or even higher cognitive processes (Cluster 2). Nevertheless, it is important to consider that this analysis does not allow excluding an earlier processing stages to index conscious perception. First, the missing significance of earlier components reflecting conscious perception, for example visual awareness negativity, could be attributed to their relative lower effect compared to the large effect of later components, for example LP or P3 (Koivisto \& Revonsuo, 2010). Thus, it cannot be completely ruled out, that differences in neural processing at earlier time ranges went unnoticed, due to the type of analysis. It can only be stated with that a possible earlier difference was not big enough in comparison with the large test statistic of Cluster 1 and 2 to lead to a significant result. Second, evidence of late components could be traced back to the use of a report-paradigm in the present investigation. Paradigms that used a report were criticized to identify NCCs that were confounded with higher cognitive components for example attention, working memory (for review see Tsuchiya, Wilke, Frässle, \& Lamme, 2015). These paradigms often identified later components as true NCC, instead of earlier components (Koch, Massimini, Boly, \& Tononi, 2016; Pitts et al., 2014). However, this is an ongoing debate (Overgaard \& Fazekas, 2016), with the opposite interpretation of earlier components as prerequisites of consciousness awareness (Del Cul et al., 2007; Sergent et al., 2005).

\subsubsection{Conclusion}

Differences in the neural processing depending on differences in the perception were found. The neural signatures associated with both perceptions differed in their temporal and spatial distribution and showed the reverse direction of effects. Nevertheless, Cluster 1 found for the perception of a Rotation could be generalized to the perception of a Target before Mask. However, there was still a significant difference between the perception of a Target before Mask and a Rotation. In contrast, Cluster 2 seems to be highly specific for the 
perception of a Target before Mask. The spatial and temporal similarity of Cluster 1 with later components (P3 or LP) allows the caution interpretation of this neural signature as an update of perceptual information into working memory to be processed at higher cognitive level. Whereas the even later occurrence of Cluster 2 speaks in favor for the assumption that it reflects higher cognitive demands.

In addition, evidence that differences in the perception are accompanied by distinct neural patterns even under identical physical stimulations, contradicts with the attempt to search for a global measure of awareness for metacontrast masking. Instead, without a phenomenological specification, the complexity of the target processing could be underestimated in metacontrast masking paradigms. Nevertheless, it has to be stated that only for two perceptions differences in the neural signature were found so far. Therefore, future research is necessary to provide further evidence for differences in the neural processing regarding different perceptions. 


\section{Overall Discussion}

This thesis addresses the multidimensionality assumption of metacontrast masking (Jannati \& Di Lollo, 2012; Sackur, 2013) in several steps. In a first step, the perceptual variability in the awareness of a masked target was measured by a phenomenological investigation (Experiment 1 of Chapter 3). It was examined whether naive participants were able to spontaneously perceive qualitative differences in the appearance of the target. Furthermore, it should be investigated whether the perception of the target varies qualitatively across conditions in a metacontrast masking paradigm. This would be the case if different aspects of the target depicted unique relationships with the temporal and spatial parameter. Based on metacontrast literature, the target was assume to vary regarding perceptions about the perceived temporal relation between target and mask (Target inside Mask, Target before Mask), perceptions about the perceived contrast (Dark Target, Bright Target, No Target) and motion perceptions (Rotation, Expansion). It has been shown that most of the subjects reported several perceptions spontaneously. Furthermore, each perception depicted a unique relationship with SOA and especially in the case of Rotation also with Congruency. The phenomenological results show that participants were able to describe rich and detailed visual experiences, regarding qualitative different aspects of the target. Nevertheless, the time courses of the perception showed interindividual variability, which may be traced back to differences in the experiences or in verbal or introspective skills.

To exclude some sources of variance and to replicate the time courses, Experiment 2 (Chapter 3) was performed. Therefore, subjects were trained to perceive all perceptions. The time courses of Experiment 1 could be replicated and for six of the seven perceptions they even coincide with those reported in the metacontrast literature. The time courses in dependence with the parametric variations suggest that the different perceptions represent unique perceptions in a metacontrast masking paradigm and not only trial-by-trial fluctuations in the experience. Furthermore, this investigation shows that experimental variations of the SOA or congruency influence the perception of the target not only in terms of visibility, but also in terms of qualitative differences in perception. Further, in another session objective discrimination sensitivity was captured which showed individual differences in performance, with type-A and type-B masking functions. The analysis of the relationship between subjective and objective data showed a correspondence between both measures. However, the objective data underestimates the awareness of the target particular at long SOAs, since most participants performed badly at long SOAs despite their ability to perceive several target 
aspects. Therefore, these results challenge the usage of one-dimensional objective or subjective measures which imply merely quantitative variations of the target awareness.

The multidimensionality assumption of metacontrast was not only defined by qualitative differences in the perception, but also by underlying processes associated with different perceptions (Albrecht \& Mattler, 2016; Sackur, 2013). Therefore, in a second step the question was examined whether differences in the appearance of the target provide evidence for underlying processes (Experiment 3, Chapter 4). If two perceptions are elicited by one underlying process, they should only occur together. Instead, perceptions that can be traced back to different processes should occur independently of each other. Albrecht and Mattler (2016) showed that metacontrast masking at short SOAs is based on only one process, which is associated with the perception of an afterimage. In addition, they provided evidence for two independent processes associated with the perception of a rotation and a shapeindependent visibility, both underlying long SOAs. Based on these results, it was investigated whether two perceptions which showed the same decreasing time course across SOA depended on each other: The perception of a target integrated inside the mask and the perception of an expanding target. In addition, it was assumed that the perception of a rotation and a target temporally separated from the mask, mostly occurring at long SOAs, are independent of each other. The results of Experiment 3 depict a dependency for the perceptions occurring mostly at short SOAs, whereas the perceptions at short SOAs were found to be independent of each other. This was interpreted as evidence for three processes underlying metacontrast masking. At short SOAs one process was assumed to elicit both perceptions of an expanding target and a target integrated inside the mask. At long SOAs two independent processes were assumed to elicit the perception of a rotation or a target temporally segregated from the mask, respectively. The influence of all three processes on target perception seems to be strongly determined by the SOA, whereas the congruency tended to influence at short SOAs which perception was more perceived.

In a last step it was considered whether the multidimensionality assumption hitherto provided by behavioral measures, could also been supported by neurophysiological results (Experiment 4, Chapter 5). For the two perceptions that already showed independence of the basis of behavioral data, it was investigated whether evidence for different neuronal processes can be found. Event-related potentials (ERPs) of trials on which participants perceived a rotation respectively a temporally separated target were contrasted with trials on which these perceptions were not perceived. Despite identical physical stimulation, two distinct neural signatures were found for both perceptions. This provided neurophysiological evidence for 
different processes underlying metacontrast masking, since the neural signatures diverge in their temporal and spatial extent. Cluster 1 representing the neural signature of the perception of a rotational movement was found at an earlier $(\sim 230-550 \mathrm{~ms})$ but overlapping time window compared to Cluster $2(\sim 530-840 \mathrm{~ms})$, representing the neural signature of the perception of a temporally separated target. For both clusters a large network was found which differed in its spatial-temporal dynamic, since only Cluster 1 exhibited exclusive frontal-parietal activity at intermediate time ranges. In addition, the opposite direction of effects was found for both clusters. For Cluster 1 larger amplitudes were found for trials on which a rotation was perceived. In contrast, for Cluster 2 amplitudes were higher for trials on which no target was perceived. Evidence for the differentiability of the two neuronal signatures is also shown in the specificity of the clusters. Cluster 2 was highly specific for the perception of a temporally segregated target, whereas Cluster 1 was at least partially generalizable to the perception of a segregated target. Nevertheless, another indication for the distinctiveness of both clusters was provided by significant differences of ERPs between the perception of a rotation and a segregated target. Therefore, behavioral and electrophysiological results depict evidence for distinct processes which were related with differences in the phenomenology.

\subsection{A methodological evaluation of the phenomenological investigation}

Despite this criticism to consider subjective data, at the beginning of the 21 st century there were numerous demands to embed introspective or phenomenological methods in an experimental setting. In the general introduction (Chapter 1) two different approaches were presented that made the attempt to introduce a method to naturalized Phenomenology. These methods were inspired by neurophenomenological (Varela, 1996; Varela \& Shear, 1999) and heterophenomenological approaches (Dennett, 1991, 2003, 2007). In this thesis both approaches were combined in two consecutives steps, since this provide a promising approach to fulfill phenomenological requirements embedded in experimental paradigms of cognitive psychology. In the following, it is discussed in detail to what extent the investigations of this thesis fulfill or extend the methodological requirements of a naturalized phenomenological investigation.

\subsubsection{Neurophysiological approach}

Four aspects were designated as necessary prerequisites for a successful methodological implementation of neurophenomenological investigations (Varela, 1996). 
This so-called phenomenological reduction should preserve the subjective aspects of the data and not already transform them into third-person, quantitative data (Gallagher \& Sørensen, 2006). The requirements of the phenomenological reduction (Varela, 1996) were met in Experiment 1. In addition, the methodology was evolved and standardized to improve the data collection and to avoid the problems of the first phenomenological or introspective investigations.

First, an intense training was proposed to achieve stability in the perception. Therefore, Experiment 1 included three training sessions.

Second, as the basic attitude for phenomenological experiments, subjects were asked to focus only on the experience itself. This requirement was met as participants were instructed to describe their experiences in as much detail as possible without interpreting them. This means subjects were asked to exclude thoughts, feelings or associations inspired by the perception. Instead, they had to focus only at their experiences without formulating a theory or opinion about it.

The third requirement of the phenomenological reduction was to gain intimacy with the own experience. In Experiment 1, particular emphasis has been placed on providing a broad context of visual experiences through variations of temporal and spatial parameters. This context should allow the subjects to gain as much experience as possible with the visual material.

In addition, a rich context of experiences should promote the awareness of differences in the perception. As demanded in the fourth step of phenomenological reduction, participants should be able to formulate communicable commonality. A purely passive observation was not considered sufficient. Instead, continuous reports of experiences were considered to be so important for the acquisition of communicable commonality that subjects were asked on each trial of the training sessions to describe their individual perception. Further, at the end of each session participants had to describe and sketch their perception. This survey was conducted to ensure that subjects identify and name differences in experience and become familiar with their own recurring impressions. These steps were in accordance with the methodology of Lutz et al. (2002), whose investigation was termed as the first successful implementation of the phenomenological reduction in an experimental setting (Gallagher, 2003).

In the next step of Experiment 1, individual descriptions were rated into predefined categories, to observe similarities in the time course of the individual experiences across conditions. But in contrast to Lutz et al. (2002), the categorization has not been done by the subjects itself, but by raters, who were naive according to the hypotheses and the design of the 
experiment. Gallagher (2003) criticized the usage of pre-established, hence, objective categories, which washes out the first-person perspective. However, as Gallagher (2003) stated does this procedure fulfill the requirements of a phenomenological investigation. Since the predefined categories of the present investigation were based on previous studies about qualitative different experiences in metacontrast masking paradigms, they can be interpreted as phenomenological data from a secondary source.

Experiment 1 of this thesis also represents a further development of the neurophenomenological method. Early introspective or phenomenological investigations were discredited as unreliable and difficult to falsify, because their results rely on private inner states, only the subject has access to (Velmans, 2007). This problem occurs whenever there is no variation in the experimental conditions, as in the study of Lutz et al. (2002). Variations can be used to show whether differences in phenomenology depend on stimulus conditions or only on trial-by-trial fluctuations in the perception caused by differences in the inner state of the participant. Thus, it is possible to falsify the phenomenological descriptions, by identifying interindividual commonalities in the perceptions that showed accentuated time courses across stimulus condition. This ensures that the categories rather summarize intersubjective valid perceptions, varying with the experimental conditions, than focusing on intraindividual variability in the perception. Nevertheless as a limit of the falsification, it cannot be determined from a third-person perspective whether a perception is true or based on an illusions. Thus, one might not deny the observers correctness of his first-person perspective. Instead, one could evaluate from the third-person perspective whether the particular perception is a perception of interest, because it is shared by others and depends on stimulus conditions.

\subsubsection{Heterophenomenological method}

If the present investigation would only base on the neurophenomenological method, the results would be only conditionally meaningful and characterized by methodological weaknesses. In Experiment 1 participants were not influenced in their verbal descriptions or their focus of attention and differed in terms of their introspective abilities. This resulted in a high interindividual variability independent from the experimental variations. This may be a common methodological problem of earlier introspective or phenomenological studies that suffered from a lack of reproducibility. These problems were mitigated by the heteronomous method applied in the other experiments of the present thesis. Based on demand of 
Heterophenomenology, the individual descriptions of the participants were regarded as the raw data which needed to be interpreted in a meaningful way (Dennett, 1991). Commonalities in the individual descriptions, which were classified into the same perceptual category, were used to formulate prototypical descriptions of experiences. These descriptions were used to train participants to focus on the perceptual categories captured in each experiment (Experiment 2-4).

As another methodological particularity, great emphasis was placed on detailed feedback. To achieve this, in the training sessions participants were requested to describe periodically what they have had perceived previously. The experimenter was trained to recognize small differences in the descriptions that might indicate misunderstandings regarding the definitions of the perceptions. In addition, this should help to focus the attention on the central aspects of the perceptions. Thereby, the experimenter took the attitude, what was described as adopting the intentional stance (Dennett, 1991). This means, the investigator has to interpret the description given by the subjects to discover the intentionality of the reports. Dennett (1991) highlighted the pitfall that subjects may rather report whatever they believed the investigator wants to hear, instead of reporting what they perceive. To avoid this problem, an atmosphere was created were the participants felt free to report whether they have or have not perceive a perception. Furthermore, they were asked to report their experiences in their own words and not just to repeat the given definitions. This was supported by further questions concerning the perceptions, which had the goal to give the subjects a better idea of what they had seen.

In Experiment 2-4 training was followed by sessions in which participants were asked to indicate their perceptions by button press. Even if this procedure no longer corresponds to the principles of a pure phenomenological investigation, it ensures the implementation of subjective measurements in a scientific framework. On the one hand, this procedure allows to replicate the time course of the perceptions, whereby some sources of interindividual variance were avoided by specifying the perceptual categories (Experiment 2). On the other hand, standardization makes it possible to associate perception with cognitive (Experiment 3) or neurophysiological processes (Experiment 4).

\subsubsection{Methodological limitations and summary}

Nevertheless, any phenomenological methodology has disadvantages, regarding the time and cost consuming procedures caused by the intense training. In the present 
investigation the training poses great challenges to the instructor and the subjects. The latter had to observe very subtle and difficult to perceive variations in the appearance of the target stimulus. Despite the efforts to standardize the procedure, many sources of unexplained variance remained. This is mostly due to the fact phenomenological data has the inherent problem of subjectivity which implies a greater variability than objective data. In addition, subjective measures have been criticized because differences found between participants may not due to differences in the perception, but caused by differences in the response bias (Wiens, 2008). Consequently, based on the idea of the signal detection theory (Macmillan \& Creelman, 1991), the willingness to affirm a corresponding perception varies between participants. This criticism also applies to the present investigations, although it was attempted to define the criterion content by the instructions of the perceptions. For example, for the perceptions of a target integrated inside the mask, it was pointed out that neither the shape of the target needs to be perceived, nor does the target needs to be perceived as dark as it appears as unmasked object. This attempted to unify the criterion content of the subjects in order to ensure that this source of variance was minimized. Nevertheless, differences in the response bias between the subjects cannot be completely avoided and represent a source of unexplained variance also in the present investigations.

To sum up, the investigations of the present investigation fulfilled the principles of a phenomenological investigation. At the same time this method was embed in an experimental psychological setting. This implementation of a naturalized Phenomenology was strongly oriented to the methodological demands of the Neurophenomenology (Varela, 1996; Varela \& Shear, 1999) and the Heterophenomenology (Dennett, 1991, 2003, 2007). Despite the problems of these phenomenological methods, this approach proved superior to earlier phenomenological investigations, as some of the common problems that led to the discrediting of phenomenological studies were avoided.

\subsection{Phenomenological insights into metacontrast masking}

There is evidence to consider metacontrast masking as a multidimensional phenomenon (Sackur, 2013). The multidimensionality of metacontrast masking is revealed by varying perceptual experience of the target across SOA (Kahneman, 1968). In accordance, the descending and ascending branches of the metacontrast function were associated with differences in the perceptions of the target (Jannati \& Di Lollo, 2012; Neumann \& Scharlau, 2007; Reeves, 1982; Sackur, 2013). However, Experiment 1 and 2 of this thesis are the first 
phenomenological investigations that systematically examine the appearance of the target in dependence of the temporal and spatial relations between target and mask. The results indicated that the seven percepts extracted from metacontrast literature represent reliable perceptions of the target, even occurring together in one paradigm. Since each category represents a different aspect of the target experience, this provides evidence for the richness of experience under metacontrast masking:

First, with increasing SOA a decrease in the perception of a target integrated inside the mask was found. This is in accordance with the evidence that the integration mechanism, which was proposed to underlie short SOAs, declines with increasing SOA (Eriksen \& Rohrbauch, 1970; Francis \& Cho, 2008; Neumann \& Scharlau, 2007; Reeves, 1982; Scheerer, 1973; Scheerer \& Bongartz, 1973). Because metacontrast masks spatially surround the targets without overlapping contours, the integration of target and mask leads to a better visibility (Francis \& Cho, 2008). Therefore, the integration of target and mask was proposed to result in a superposition of both stimuli to one composite, with a well visible target.

Second, with increasing SOA the perception of a target temporally segregated from the mask was found. This time course is in accordance with the assumption of a segregation mechanism which increases with the temporal distance between target and mask (Neumann \& Scharlau, 2007; Reeves, 1982). These authors provided evidence that with increasing segregation, the visibility of the target rises, because both stimuli can be separated perceptually from each other. Nevertheless, in the present investigation also at short SOAs a segregated target has been reported, resulting in a slight $\mathrm{u}$-shaped time course across SOA. This indicates the difficulty to differentiate between an integrated and segregated target. Previous studies differentiated between these two perceptions, simplified the task by using much longer SOAs compare to the present investigations and forced participants to choose between these two percepts (Neumann \& Scharlau, 2007; Reeves, 1982). Therefore, the rather short range of SOAs used in in the experiments of this thesis could impede the perception of a segregated target. Further, since both perceptions were evaluated independently, it was much more difficult to distinguish between them. Consequently, the u-shaped time course found for the perception of a segregated target may be explained by the high probability to affirm both perceptions on each trial a dark target was perceived, regardless of the perceived temporal relation.

Third, the perception of a dark target yielded a u-shaped function across SOA, which confirms previous findings (Breitmeyer et al., 2006; Neumann \& Scharlau, 2007; Werner, 1935). 
Fourth, the appearance of a brightness reversal has been reported in metacontrast literature, with a black target appearing whiter than the background under metacontrast masking (Heckenmueller \& Dember, 1965a; Purcell \& Dember, 1968; Stewart et al., 2011; Werner, 1935). Stewart et al. (2011) provided evidence that a brightness reversal appears at short SOAs. In Experiment 1 of this thesis subjects spontaneously reported to perceive a brightness reversal, nevertheless no clear relation with SOA or congruency was visible. In Experiment 2 only a slight decrease with SOA was found. The unspecific time course can be traced back to the task, since evidence for a brightness reversal was only found with a spatial forced-choice task, but not with a temporal forced-choice task (Stewart et al., 2011). Thus, it might be concluded that a measurement of the perceived brightness with a centrally presented stimulus without spatial comparison is not an adequate method for determining the time course of a brightness reversal.

Fifth, in accordance with previous results a reverse u-shaped time course for the perception of metacontrast suppression was found. At intermediate SOAs the perception of a phenomenally absent target was found for flashes of light as stimuli (Alpern, 1953; Fehrer \& Raab, 1962; Kahneman, 1967; Weisstein \& Growney, 1969), dark stimuli on bright background (Stewart et al., 2011), as well as for stimuli with opposite contrast polarity on a uniform gray background (Breitmeyer, Tapia et al., 2008).

Sixth, apparent motion perceptions were reported in the metacontrast literature. For target-mask combinations with different spatial layouts, the perception of a rotation was found, which resulted from the interplay of shape incongruent target-mask sequences and occurred mostly at long SOAs (Albrecht \& Mattler, 2012b, 2012a; Ansorge et al., 2009; Maksimov et al., 2011). In accordance, an increasing time course at incongruent trials and the constant low frequencies of reports at congruent trials have been found reliably at all experiments reported in this thesis.

Seventh, for target-mask combinations without congruency manipulations, for example rectangle or ring-disc shaped stimuli, another apparent motion perception was found, which was described as an objectless enlargement (Fehrer \& Raab, 1962; Hogben \& Di Lollo, 1984; Kahneman, 1967; Toch, 1956; Weisstein \& Growney, 1969). This perception was strongest at intermediate SOAs around $50 \mathrm{~ms}$, where the metacontrast suppression was maximal (Kahneman, 1967; Weisstein \& Growney, 1969). In contrast to these results, in the present investigations an expansion has been reported mostly at short SOAs, where discrimination performance was found to be maximal (Experiment 2). Not only the time course, but also the phenomenological description of an expansion as an enlargement of the 
target itself, differed from the reports of an expansion as an objectless enlargement found in metacontrast literature. Because of these differences it is plausible to assume that the expansion of the present investigations cannot be equated with an apparent motion phenomenon. Instead, the expansion is phenomenological similar to the description of a filling-out process of surface contrast (Breitmeyer \& Jacob, 2012). Breitmeyer and Jacob (2012) defined filling-out as a continuous surface completion, which takes some time to be completed. In accordance, an expansion was described as the impression of dark point growing inside the mask and resulting in a contrast gradient between the center and the border of the target. Further considerations on how to interpret the perception of an expansion are discussed in section 6.3.2.

The validation of the seven perceptions is based not only on the correspondence between the time courses found in the present investigation and those reported in metacontrast literature, but also on the high agreement between the time courses of Experiment 1,2 and 3. Therefore, the perceptions seemed to reflect reliable differences in the appearance of the target. The accentuated and different time courses of the categories highlighted the fact that the perceptions were not just a result of trial-by-trial fluctuations in the awareness and therefore not caused by noise in the perception. Instead, they seemed to represent unique aspects of the target. Since each perception yielded a distinct relation with the spatial and temporal parameters, it could be assumed that the perception of the target differs qualitatively across conditions in a metacontrast masking paradigm. The modulation of the perception as a function of the experimental manipulations, promoted the view that our representation of the environment is actually rich and detailed and refutes the criticism, introspection is illusory or even delusional (Haun et al., 2017).

\subsubsection{Subjective experience and their relation to objective performance}

Individual differences in discrimination performance were found with some participants showing a constant low performance across SOA and others showing an increasing performance with SOA. However, most of the individual masking functions depicted a decreasing time course across SOA, resulting in a decreasing curvilinear masking function on average. The interindividual variability in performance level or slope confirmed previous results (Albrecht et al., 2010; Albrecht \& Mattler, 2012b, 2012a, 2016; Fleischhauer et al., 2014; Maksimov et al., 2011). 
In the present investigation a double dissociation was found, since several perceptions yielded high frequencies at long SOAs, whereas the averaged discrimination performance decreased with SOAs. At the late range of SOAs close to zero awareness was indicated by the objective measure, whereas subjective measures suggested the perception of several target aspects. The discrimination performance was captured in a separate session, after seven sessions of perceptual learning in the subjective task. It is notable how much the average objective performance laged behind the subjective experience. Consequently, the perceptual cues gained in the subjective task were not transferred to discriminate the target, especially at long SOAs. There are many inconsistencies regarding transfer effects between subjective and objective tasks in masking paradigms.

On the one hand, training effects in an objective task were found to generalize to SOAs that were not shown in the training session and lead to an improvement in the subjective awareness (Schwiedrzik et al., 2009). On the other hand, there is evidence that trainings effects do not generalize between an objective and subjective task. Training in an objective task resulted in an improvement to discriminate between fearful and non-fearful faces, whereas subjective awareness did not change by training (Szczepanowski \& Pessoa, 2007). Furthermore, blindsight goes along with the subjective impression to experience nothing within the visual field contralateral to the damaged hemisphere in the visual striate cortex. However, above-chance perform was found in a visual forced-choice task (Weiskrantz et al., 1995). A blindsight patient showed training effects in an objective form-discrimination task, which did not generalize to the subjective impression of only guessing (Trevethan, Sahraie, \& Weiskrantz, 2007). Consequently, despite the same visual stimulation and the great similarity of both tasks to indicate awareness, transfer effects between subjective and objective measures were often missing. Training effects across SOAs in a masking paradigm were explained by a shifs in the criterion content where participants learned to differentiate variations in the appearance of the target (Ventura, 1980). The missing transfer effects suggest that the task-relevant information needed to accomplish both tasks seems to be different.

Qualitative differences in objective performance were associated with the usage of different perceptual cues to perform the objective task (Albrecht \& Mattler, 2012b, 2012a, 2016). Also in the present investigation relationships between the perceptual categories and the discrimination performance could be found with most perceptions showing a unique relationship with discrimination performance. The perceptions of a temporally segregated target, a dark target and the inverse time course of a perceptually absent target (No Target) showed general relationships with the discrimination performance. Whereas the perception of 
a target integrated inside the mask and a bright target helped to discriminate the target shape mostly at short SOAs, even if the contribution of a bright target was only marginal. In accordance with the results of previous studies (Albrecht \& Mattler, 2012a; Ansorge et al., 2007; Ansorge et al., 2009; Maksimov et al., 2011), the perception of a rotational movement helped to discriminate the target at long SOAs and on incongruent trials. The perception of an expanding target had no influence on the discrimination performance at all.

The results of Experiment 2 indicated that the inability of the participants to discriminate the target at long SOAs was not solely due to an impairment of perception. The majority of participants produced Type-B masking functions, even if the perception of a rotation yielded the expected time course in the preceding sessions with the subjective task. This shows that most participants could theoretically distinguish between congruent and incongruent trials by defining a rotation as an indication of an incongruent trial. In contrast, most of the participants seemed to be unable to transfer this strategy to the later discrimination session. This speaks in favor for the assumption that the low discrimination performance at long SOAs was not related to an absence of phenomenological experience, but the inability to use the trained perceptual cues.

Training and transfer effect in perceptual tasks were explained by the Reverse Hierarchy Theory (Ahissar \& Hochstein, 1997, 2004). The Reverse Hierarchy Theory was also used to explain the stability of the individual masking functions (Albrecht et al., 2010). Individually different metacontrast masking functions were found to remain stable even after training (Albrecht et al., 2010; Albrecht \& Mattler, 2012a). This indicates that participants were not able to generalize training effects across SOA. In accordance, participants were not able to use a perceptual cue, different to their original one, even if they were instructed to do so (Albrecht \& Mattler, 2012a). Albrecht et al. (2010) proposed that participants select one of the two neural levels to perform the task based on their individual predisposition, already acquired before training. In dependence of the chosen neural level, Type-A or Type-B masking functions resulted. The assumption of the Reverse Hierarchy Theory is expanded by the absence of transfer effects between subjective and objective tasks indicated by pervious results and the double dissociation found in the present investigation.

These results conflict with Albrecht et al. (2010) assumption about one individual predisposed neural level to perceive the masked target. Under this assumption, similar performances for subjective and objective tasks would have to be shown. Even if the individual ability to perceive the different target aspects were accompanied by an increased performance, on average objective performance lagged behind subjective perception. 
Therefore, the neural level for processing visual information seems to be not only defined by the complexity of the stimuli, but also by the exact task.

The low discriminatory performance at long SOAs is all the more astonishing, since congruent and incongruent trials could be distinguished at long SOAs, as the time course of the rotation indicates. However, transfer effects were found to be dependent on whether the trained cue can be used similar in both tasks (Ahissar, Nahum, Nelken, \& Hochstein, 2009). Ahissar et al. (2009) compared two investigations of peripheral crowding, with letters as target and distractor stimuli which differed only in the exact task. When all of the three letters had to be reported, learning as well as transfer to peripheral reading was found (Chung, Legge, \& Cheung, 2004), whereas no transfer occurred if only the central letter had to be reported (Chung, 2007). In accordance to the assumption of Ahissar et al. (2009), only the three letter task required the same visual constrains as peripheral reading. The usage of a rotation to discriminate the target requires both, the understanding that the impression of the movement originates from the incongruence of both stimuli and that the shape of the mask must be taken into account to derive the shape of the target. Consequently, the training to perceive a rotation does not provides a direct strategy to discriminate the target in the objective task.

Summary. Despite the inability of most participants to use the perceptions at long SOAs to discriminate the target, at short and long SOAs a correspondence between subjective experiences and discrimination performance existed. Since perception cohered with the ability to discriminate the target, this validated the perceptual categories as unique experiences in a metacontrast masking paradigm. The lack of correlation for the perceptions of an expanding target, as well as the low discrimination performance at long SOAs in combination with pronounced subjective experiences, indicates that the subjective experience of the target is much more detailed and rich as an objective measure is able to discover. In addition, the individual differences in the ability to use perceptual cues to discriminate the target indicates that an objective task must be considered with caution as a method to state about unconscious or conscious perception in a metacontrast masking paradigm. Since each perceptual category yielded a unique relation with the performance, even this seemingly simple and unambiguous discrimination task varies according to the perceptions subjects use to accomplish the task. Therefore, statements about the ability to perceive the target stimulus will differ depending on the aspect of the target subjects focus on. Further, the choice of the perceptual cue could influence which type of masking function emerge. Subjects perceive the critical stimuli in a variety of ways even in conditions when they may not able to exploit these perceptual cues to 
solve an objective discrimination task. However, to conclude that these stimuli have been "unconscious" in conditions when objective performance is low may be in fact misleading.

\subsubsection{Generalizability of the perceptions to other masking paradigms}

An open question relates to the generalizability of the seven perceptions, extracted from the metacontrast literature, to other types of masking. The assumption of a varying criterion content has only been proposed for metacontrast masking (Kahneman, 1968). Nonetheless, evidence for qualitative differences in the perception of the target across SOA can also be found for other types of masking paradigms, indicating that the rich phenomenology found for metacontrast masking can be generalized at least in part.

Paracontrast. Paracontrast is a special kind of forward masking, where the mask precedes the target in time and both stimuli do not overlap spatially, but show adjacent contours (Alpern, 1953). In comparison to metacontrast, weaker masking effects were found for paracontrast (Alpern, 1953; Growney et al., 1977; Kolers \& Rosner, 1960).

For paracontrast evidence for variations in criterion content can be deduced from different masking functions in dependence of the exact task. Type-B masking functions were obtained with brightness or contrast judgment tasks (Kolers \& Rosner, 1960), whereas TypeA functions were found for detection tasks (Lefton \& Newman, 1976). Qualitatively different masking functions depending on the exact task indicate that different stimulus dimensions are necessary to perform the particular task. This implies that even under paracontrast, the appearance of the target is not constant, but varies qualitatively across SOA. The influence of para- and metacontrast masking on different perceptual dimensions was also investigated directly, by comparing the masking functions of a brightness and a contour judgment task (Breitmeyer et al., 2006; Stober, Brussell, \& Komoda, 1978). In a study of Stober et al. (1978) the contour clarity was more diminished at short SOAs compared to the brightness estimates, but at $-50 \mathrm{~ms} \mathrm{SOA}$ the ratings of both tasks seemed to approach to each other. Furthermore, at $-15 \mathrm{~ms}$ SOA the masked target was perceived as brighter than the target-only condition. This was interpreted as a brightness reversal perception for paracontrast. The same superiority of paracontrast masking on contour judgements compared to contrast judgments, was found by Breitmeyer et al. (2006). At short SOAs the target contour was stronger diminished by a preceding mask than the contrast, whereas both dimensions can be perceived equally well at longer SOA. In addition, contrast enhancement was found at short SOAs, which may be 
interpreted in terms of a contrast reversal. These results showed that the perception of the targets contrast is not constant across SOA in paracontrast masking, but varies qualitatively.

Standing wave illusion. Standing wave illusion describes the decreased visibility of a target which is presented in a continuous cycle with no-overlapping masks (Werner, 1935). Therefore, it can be understood as a continuous combination of meta- and paracontrast. Several different perceptions have been reported for standing wave illusion.

For paradigms with a central target bar and flankers, serving as masks, a decreasing target visibility was found with increasing ISI (0-80 ms). If even longer ISIs (100-300 ms) were used, a further increase in visibility was found, resulting in an overall u-shaped masking function (Hein \& Moore, 2010a). Therefore, comparable to metacontrast at intermediate ISI the target was phenomenologically absent, suggesting that the perception of a black target followed a u-shaped function.

In the same paradigm, increasing flanker duration led to decrease the target visibility (Hein \& Moore, 2010b) and increased strength of an apparent motion perception (Hein \& Moore, 2010b; Pilling \& Gellatly, 2009). Therefore, in accordance with metacontrast masking, the reduced visibility of the target was accompanied by the perception of an apparent motion involving the flanking bars (Werner, 1935), which was described as "an inward and outward oscillation from the location of the central bar, which is invisible and the location of the flankers, which are visible" (Hein \& Moore, 2010b, p. 407). Also this phenomenological description of an apparent motion is very similar to what has been described in metacontrast masking paradigms (Kahneman, 1967; Weisstein \& Growney, 1969).

Pattern masking. Pattern masking was defined as a spatial superposition of target and mask elements (Enns \& Di Lollo, 2000). At short SOAs an integration mechanism was proposed, which was related to the perception of a composite of target and mask (Francis \& Cho, 2008; Michaels \& Turvey, 1979; Scheerer, 1973; Spencer \& Shuntich, 1970). With decreasing integration the target became more visible because it could be perceived as being separated from the mask (Francis \& Cho, 2008).

In a forward pattern masking paradigm the subjective contrast rating was measured, relative to the contrast of a target-only condition (Bachmann, 1988). A u-shaped contrast rating across SOAs was found, with a darkening of the masked target at short SOAs and even a brightness enhancement at intermediate SOAs. In accordance, evidence for a contrast reversal was found at short SOAs in a backward pattern masking paradigm, where participants had to state whether the target appeared brighter or darker than the immediate 
background (Brussell, Stober, \& Favreau, 1978). Consequently, differences in the perceived target contrast as well as the perceived temporal distance seem to appear with pattern masking.

Vernier stimuli. Also for vernier stimuli integration masking seems to be a plausible mechanism to explain masking effects at short SOAs. Vernier stimuli are characterized by their spatial layout. The following characteristics can be found in several investigations with vernier stimuli (Dombrowe, Hermens, Francis, \& Herzog, 2009; Duangudom, Francis, \& Herzog, 2007; Herzog, Harms et al., 2003): Vernier targets usually consist of two vertical lines that are aligned with a small horizontal offset to each other. The masks are flanking lines on each side of the vernier target. Often the task is to report the direction of the targets offset, whereby the dependent variable, the offset size of the target vernier, is adapted with a staircase procedure to define the offset size where $75 \%$ correct responses are obtained. Vernier masks were also used as variants of pattern and metacontrast masks, defined by the overlap or non-overlap of the target with the central flanking bars of the mask (Dombrowe et al., 2009; Duangudom et al., 2007; Herzog et al., 2001, 2001).

For short presentation times of the vernier target, a so-called shine-through has been described as a brighter, wider, longer, flashed or superimposed perception of the vernier on the flanking bars of the mask that helped to identify the offset direction of the target (Herzog et al., 2001; Herzog, Harms et al., 2003). This perception seems to be comparable with metacontrast masking perception of a black target integrated in the center of the mask. However, the perception of shine-through strongly depended on the spatial layout of the stimuli, in particular the homogeneity of the mask (Herzog et al., 2001) and a minimum number of flanks (Herzog, Harms et al., 2003; Herzog, Lesemann, \& Eurich, 2006; Herzog, Schmonsees, \& Fahle, 2003). Such a strong influence of the spatial layout on the perceptual integration of target and mask has not been observed for metacontrast stimuli.

A first systematic investigation of the interaction between spatial and temporal parameters provided evidence for a varying criterion content with ISI for vernier stimuli (Drewes, Zhu, \& Melcher, 2014). With varying ISI between 0-100 and $200 \mathrm{~ms}$, two verniers were presented consecutively, with the offset of the second vernier shifted either in the same or the opposite direction as the offset of the first vernier. After the stimulus presentation participants had to respond amongst others whether they perceived a single vernier, a motion between both verniers or two temporally and spatially separated verniers. The perception of a single vernier was interpreted as an integration of both stimuli (Drewes et al., 2014) or as a feature fusion (Scharnowski, Hermens, Kammer, Oğmen, \& Herzog, 2007), which has also 
been termed as a case of integration masking (Rüter, Kammer, \& Herzog, 2010). The integration effect found for vernier stimuli differed from the integration of metacontrast masking, because with vernier integration only one stimulus can be perceived and not target and mask simultaneously. The motion percept of vernier stimuli was described as a flipping of the first vernier to the second vernier with the opposite offset direction (Herzog et al., 2001; Rüter et al., 2010; Scharnowski et al., 2007). It is comparable with the apparent motion phenomena found for metacontrast masking and was interpreted as a partial separation of both stimuli (Drewes et al., 2014). The perception of two separated verniers was equated with a segregation (Scharnowski et al., 2007) resulting in the perception of two successive stimuli (Rüter et al., 2010).

The time courses of the three perceptions found by Drewes et al. (2014) were in accordance with the time courses of metacontrast masking. For the integration percept a decreasing time course with ISI was found, whose slope was all the more accentuated the smaller the offset was. The motion perception provided a reverse u-shaped time course, which was enhanced with increasing spatial distance. Also for metacontrast masking an reverse ushaped time course was found for apparent motion perception (Kahneman, 1967; Weisstein $\&$ Growney, 1969). In this thesis, for the perception of a rotation only an ascending and not a $\mathrm{u}$-shaped time course was found, because the SOAs were not long enough to show the later decline of the movement experience. The perception of a segregation increased with ISI (Drewes et al., 2014), as it has been found in the present thesis for the perception of a target temporally segregated from the mask. To sum up, for vernier stimuli qualitative differences in the target perception occur, which show similarities with the phenomenology and the time course of the perceptions of metacontrast masking.

Resume. Systematical phenomenological investigations are missing for other masking paradigms. However, there is evidence for qualitative differences in the perception of the target in dependence of temporal or spatial parameters. The perception of the target differed between short and long SOAs, respectively ISIs, depending on whether an integration of target and mask took place or not. An integration either resulted in a reduced visibility which inclined with increasing temporal distance of both stimuli, as for pattern masking (Francis \& Cho, 2008; Michaels \& Turvey, 1979; Scheerer, 1973; Spencer \& Shuntich, 1970) or vernier stimuli (Drewes et al., 2014; Herzog, Harms et al., 2003; Rüter et al., 2010). But also an enhanced visibility of integrated stimuli was found for standing wave illusion, which yielded in a u-shaped function across ISI (Hein \& Moore, 2010a). Differences in the perceived contrast were found, ranging from a reduced contrast to a brightness reversal, for 
paracontrast (Breitmeyer et al., 2006; Stober et al., 1978) and pattern masking (Bachmann, 1988; Brussell et al., 1978). In addition, several paradigms provided conditions where an apparent motion between target and mask could be perceived, as for vernier stimuli (Drewes et al., 2014; Herzog et al., 2001; Rüter et al., 2010; Scharnowski et al., 2007) or standing wave illusion (Hein \& Moore, 2010b; Pilling \& Gellatly, 2009). It has to be mentioned that several of the listed masking types share spatial properties of metacontrast masking. With paracontrast masks, vernier stimuli and standing wave illusion paradigms, target and mask show adjacent but non-overlapping contours. It is an open question how the different perceptions are influenced by the spatial layout. But it can be stated that the phenomenology in masking paradigms with non-overlapping contours seems to be richer and more diverse, compared to the target experience in pattern masking paradigms with overlapping contours. These observations should be considered with caution on the basis of the collected results. In order to make more reliable statements, an experimental investigation is needed that compares the phenomenology of different masking paradigms.

It is an open question, if the different perceptions relate to different mechanisms underlying the types of masking. A systematic comparison of the target perception for different types of masking could provide a promising approach to highlight differences as well as similarities in the mechanisms accounting for different masking types. This also raises the issue of the classification of different types of masking. So far, the definition of types of masking was primarily based on spatial or temporal characteristics, such as the demarcation between metacontrast and pattern masking, which is based on the spatial relation between target and mask. Another way to define masking paradigms is based on the underlying masking mechanisms, such as in the differentiation between Object Substitution Masking (OSM) and other types of (backward) masking (Enns \& Di Lollo, 2000; Lleras \& Moore, 2003; Moore \& Lleras, 2005). According to the latter approach, instead of differentiating types of masking based on external characteristics, masking could be classified based on the underlying mechanisms (Kolers, 1983). Given the multidimensionality of experience in metacontrast and possibly in other types of masking, it can be assumed that not a single mechanism explains the masking effects over a whole range of SOAs. Instead, different mechanisms seem to be associated with differences in perceptual experience. 
6 | Consideration of metacontrast mechanisms on the basis of phenomenological data

\subsection{Consideration of metacontrast mechanisms on the basis of phenomenological data}

The phenomenological insights into metacontrast masking demonstrated the richness and diversity of the experience in metacontrast masking and provided evidence that metacontrast masking is not a unitary phenomenon (Albrecht \& Mattler, 2016; Fahrenfort et al., 2007; Sackur, 2013). Methodological problems resulting from the lack of consideration of phenomenological variability have already been discussed in Chapter 6.2. Beside these issues, also statements about underlying processes may be subject to errors if the phenomenology is considered insufficiently. For example Pilling and Gellatly (2009) argued that the phenomenology provide hints to the masking processes in a standing wave illusion paradigm and thus rejected Enns's (2002) interpretation, who postulated a unitary mechanism. Pilling and Gellatly (2009) provided evidence that the temporal relation between target and mask determinates how much apparent motion is perceived. In addition, in conditions were less apparent motion was perceived, no coherence between shape similarity and visibility was found, instead the visibility related to the amount of adjacent contour.

Based on their findings Pilling and Gellatly (2009) questioned the earlier interpretation of Enns (2002), that reentrant processing is necessary to explain why physically similar target-mask combinations depict larger masking effect than dissimilar ones. Instead, they concluded that only in conditions were apparent motion is perceived the coherence between target-mask shape similarity and visibility may be due to reentrant processing. But in conditions where the visibility was related to the amount of contour, they could not rule out lateral inhibition as masking mechanism.

This juxtaposition aims to clarify the importance of phenomenological data for inferences about underlying processes. In contrast, most of the metacontrast models did not take into account differences in the appearance of the target explicitly, except an extension of the RECOD model (Breitmeyer et al., 2006) and the development of the Perceptual Retouch Theory (Bachmann \& Kirt, 2013; Kirt \& Bachmann, 2013). The multidimensional assumption of metacontrast masking also contradicts with the proposition of a single mechanism being able to explain metacontrast masking, as proposed for example by lateral inhibition models (Bridgeman, 1971, 2001; Francis, 1997; Macknik \& Livingstone, 1998; Macknik \& MartinezConde, 2004, 2007; Stigler, 1910; Weisstein, 1972).

However, the third experiment of the present thesis provided evidence for different processes underlying metacontrast masking, which were associated with differences in the appearance of the target. Consequently, this result expanded the conceptualization of 
metacontrast by providing evidence for three processes, two of them underlying the late branch of the metacontrast function, which has only been assumed by very few models (Albrecht \& Mattler, 2016; Michaels \& Turvey, 1979).

The assumption that different processes underlying metacontrast are associated with differences in perception was also confirmed on the basis of electrophysiological data (Experiment 4). Despite identical physical stimulation evidence for two different neuronal signatures were found, which were accompanied by the perception of a rotational movement and a target temporally segregated from the mask. Differences in the neural processing on the basis of phenomenological data, contradicts with the attempt to identify a global NCC based on one-dimensional subjective or objective measures (e.g. Del Cul et al., 2007; Lamy et al., 2008; Railo \& Koivisto, 2009; Rutiku et al., 2015; Wilenius \& Revonsuo, 2007). Instead, the results of Experiment 4 expand the multidimensionality assumption of metacontrast masking based on behavioral data to a neurophysiological level. Implications for the consideration of underlying processes, which result from the assumption of multidimensionality of metacontrast masking, are outlined below.

First, this assumption illustrates the difficulty to contrast different conditions in a metacontrast masking paradigm to search for neural correlates of consciousness (NCC). Several studies used the contrast of different SOAs or masks (e.g. Koivisto et al., 2006; Railo \& Koivisto, 2009; van Aalderen-Smeets et al., 2006) to state about NCCs, without considering differences in the phenomenology. However, the results of Experiment 1-3 provided striking evidence that the perception of the target varies qualitatively with the spatial and temporal relationship between target and mask. Consequently, the contrast of short and long SOAs is not only accompanied by differences in objective performance, but also by qualitative differences in the target perception. Differences in the phenomenology were found to be associated with differences in the neural processing (Experiment 4). Therefore, findings that differences in the target experience were accompanied by differences in the neuronal processing, question the goal of identifying a unitary NCC in metacontrast masking paradigms. Further, the contrast of conditions associated with distinct perceptions, implies the contrast of ERPs that do not reflect gradual differences in awareness, but qualitatively differences in the neuronal signature.

Second, an established procedure for the study of NCCs is based on the assumption that an additive effect of the mask on the target processing can be cancelled out by subtracting ERPs of the target-mask condition from ERPs of the mask-only condition (Del Cul et al., 2007; Railo \& Koivisto, 2009). In contrast, qualitative differences in the perception contradict 
with the assumption that the neural processing of target is only affected additively by the mask. Instead, perceptions which imply an interplay between target and mask, for example the perception of a rotation, make it reasonable that target and mask interact with each other. Contra evidence for the additivity assumption is also provided by the results of Experiment 4. Two Clusters 2 were found which differ in temporal and spatial extend, as well as the direction of effects. This suggests that the neural signatures also reflect qualitative differences in the processing. It can be assumed that ERPs of masked targets do not only reflect an additive effect between both stimuli, which speaks against the premise of a subtraction method.

Finally, since subjects do not only vary in their ability to discriminate or identify the target, but also in the usage of the perceptual cue they based their performance on (Albrecht et al., 2010; Albrecht \& Mattler, 2012b, 2012a, 2016), interindividual differences in the neural processing may accompany metacontrast masking. This could be problematic in the case of one-dimensional measures which under-specified the perceptual content, participants should focus at.

The necessity to consider differences in the phenomenology in a metacontrast masking paradigm concerns theories about underlying process. Without this specification, assumptions about underlying processes may be oversimplified. Further, several methodological problems may arise. In the following sections, a conceptualization of metacontrast masking is proposed based on behavioral and neurophysiological data that corresponds to the multidimensionality assumption, in terms of phenomenological diversity and its relationship to underlying mechanisms.

\subsubsection{Processes underlying metacontrast masking}

Based on the first two experiments of this thesis no statement can be made about underlying processes. Since each perception was captured independently of the others, it remained unknown whether different perceptions were elicited by separate processes or if one process is responsible for multiple perceptions. In the third experiment of this thesis it was examined, whether perceptions yielding the same time course at short and long SOAs, could be attributed to the same processes or were elicited by two different processes. Conclusions about the number of processes at the ascending and descending branch of the metacontrast function should be drawn on the basis of independence respectively dependence of the perceptions on each other. In Experiment 3 the perceptions of an expanding target and the 
perception of a target integrated inside the mask both yielded a declining time course and were found to depend on each other, which was interpreted as evidence for one process underlying metacontrast masking at short SOAs.

This assumption is in accordance with the results of Albrecht and Mattler (2016), who found evidence for one process underlying metacontrast masking at short SOAs, which they termed Type-B process. Based on previous results (Albrecht et al., 2010; Albrecht \& Mattler, 2012b, 2012a) the Type-B process was associated with the perception of an afterimage, which was assumed to contribute to the target discrimination at short SOAs (Albrecht \& Mattler, 2016) and was strongly related to the phenomenological description of a target integrated inside the mask. In addition, in Experiment 3 two perceptions with an increasing time course were found, a rotational movement and a target preceding the mask. Both occurred independent of each other, which was taken as evidence for two independent mechanisms. This result confirmed the conclusion of Albrecht and Mattler (2016), who proposed two independent processes underlying long SOAs, which have been termed as Type-A and TypeC process, respectively. Albrecht and Mattler (2016) used different stimulus sets that were modified to exclusively hinder one of the perceptions, which were assumed to serve as criterion contents to perform the discrimination task at short or long SOAs, respectively. The authors speculated that first-order luminance- or contrast-defined stimuli are responsible for the perception of an afterimage. Therefore, the usage of second-order texture- or movementdefined stimuli with a uniform luminance of object and background should eliminate an afterimage. According to their hypotheses, the influence of the Type-B process on the metacontrast function declined with second-order stimuli, resulting in a decrease of performance at short SOAs. This can be interpreted as evidence that the usage of stimuli defined by contrast differences is essential for the target visibility at short SOAs.

Luminance-defined stimuli were assumed to be processed within V1, whereas the processing of second-order texture-defined stimuli was located in V2 and movement-defined objects in V4 (for review see Orban, 2008). The low target visibility at short SOAs using second-order stimuli can be interpreted as evidence that the metacontrast effect on the target processing takes place in a lower hierarchical level, necessary for the processing of secondorder stimuli. In contrast, with second-order stimuli Type-A metacontrast functions were still found (Albrecht \& Mattler, 2016; Sackur, 2011; Vernoy, 1976) which speaks in favor for the idea that the target processing at long SOAs takes place in higher areas of the visual hierarchy, compared to short SOAs. Texture-defined stimuli were assumed to eliminate lowlevel lateral inhibition mechanism, making it possible to examine the role of higher-level 
6 | Consideration of metacontrast mechanisms on the basis of phenomenological data

mechanism like reentrant processing, which was found to be interrupted under backward masking, whereas the early feedforward sweep was left intact (Fahrenfort et al., 2007). Masking effects with contrast-defined stimuli were assumed to include lateral inhibition mechanism (Fahrenfort et al., 2007; Vernoy, 1976), which are among others responsible for contour formation or contrast perception (Weisstein, 1968).

However, with texture-defined stimuli also Type-B masking functions were found (Tapia, Breitmeyer, \& Jacob, 2011). These differences in results may be explained by differences in design of the stimuli. While the texture-defining stimuli, which yielded Type-A functions (Sackur, 2011; Vernoy, 1976) were composed of pixels, Tapia et al.'s (2011) stimuli consisted of line elements that, due to their length, tended to give the impression of a contour by contrast differences and therefore could rather worked as first-order stimuli.

To sum up, there is evidence that low-level mechanisms, mainly lateral inhibition, are responsible for metacontrast masking effects at short SOAs, whereas higher-level mechanism can be assumed to take place at long SOAs. In the next two sections, this will be discussed in more detail, including the considerations how far the particular perceptions examined in Experiment 3 fit into the assumption of low-level or high-level mechanisms and what kind of high-level mechanisms must be assumed to explain two independent perceptions at long SOAs.

\subsubsection{Low-level masking mechanism at short SOAs}

As outlined in the Introduction (1.3.2) several masking models proposed lateral inhibition being responsible for metacontrast masking at a whole range of SOAs (Bridgeman, 1971, 2001; Francis, 1997; Macknik \& Livingstone, 1998; Macknik \& Martinez-Conde, 2004, 2007; Stigler, 1910; Weisstein, 1972). However, none of these models took into account differences in the appearance of the target. Only Bridgeman (1971) considered the effect of the criterion content on the masking function. But his model had come under criticism by Weisstein et al. (1975). Weisstein et al. (1975) provided an simulation of Bridgeman's (1971) model, based on which they concluded that Bridgeman's simulation was to coarse and considered not enough time points to predict the metacontrast function properly. Therefore, it is questionable how adequate Bridgeman's (1971) model is to explain metacontrast masking in general.

Even though lateral inhibition mechanisms seem to be unsuitable for explaining the whole range of perceptions of metacontrast masking, these models show a high predictive 
power in simulating effects of experimental manipulations on the metacontrast masking function at short SOAs (Bridgeman, 1971; Francis, 1997; Weisstein, 1972). Thus, as described below, lateral inhibition mechanisms are sufficient to explain the influence of experimental manipulations on the metacontrast function at short SOAs. First, Francis's (1997) lateral inhibition model was able to simulate that duration differences of target and mask affected the metacontrast function at short SOAs (Breitmeyer, 1978a; Merikle, 1977; Neumann \& Scharlau, 2007). Francis (1997) proposed that an excitatory feedback elicited by the presentation of the target allows to maintain the target activity even after its presentation. The strength of the excitatory feedback determines the influence of the inhibitory activity elicited by the mask. Second, stronger masking effects were obtained with more contour of the mask at $0 \mathrm{~ms}$ ISI (Sherrick \& Dember, 1970), which has been simulated by the lateral inhibition models of Bridgeman (1971, 2001) and Francis (1997). Third, lateral inhibition models were able to simulate that the shape of the masking function changed from Type-B to Type-A with decreasing luminance contrast of the target under constant luminance contrast of the mask (Bridgeman, 2001; Francis, 1997; Weisstein, 1972). Weisstein (1972) explained variations in the masking functions based on luminance differences by changes in the inhibitory effects of the masking signal compared to the excitatory signal of the target. Based on these results it seems reasonable to assume lateral inhibition as the appropriate mechanism for metacontrast masking at short SOAs.

Furthermore, the principle of lateral inhibition is well suited to explain the perceptions at short SOAs, examined in this thesis. It has been shown that the inhibitory contour interactions diminished the contrast of the target stronger at the border of the stimulus close to the mask, compared to the parts of the target, which were farer away from the mask, for example the center of the target (Petry, 1978; Werner, 1935). A simulation of the inhibitory effects of the mask on the target showed that erosion of the target signal started at the contour, whereas the luminance or contrast of the target center was affected less, resulting in a contrast gradient from the center to the border of the figure (Francis, 1997). In accordance, in Experiment 1 an expanding target was described as a perception of a small point or circle, which starts in the center of the mask and expanded outwards until it fits into the inner contour of the mask. In accordance, some authors investigated a surface completion process underlying metacontrast at short SOAs (Bachmann, 2009b; Breitmeyer et al., 2006; Paradiso \& Nakayama, 1991; Stoper \& Mansfield, 1978), which was described as a filling-in or fillingout process of target's contrast that is not completed immediately but takes some time and can be disrupted by a following mask (Breitmeyer \& Jacob, 2012; Paradiso \& Nakayama, 1991). 
6 | Consideration of metacontrast mechanisms on the basis of phenomenological data

This process was assumed to result in a gradual, difficult to localize transition between the dark center and the lighter border of the target (Stoper \& Mansfield, 1978). Consequently, the lateral inhibition mechanism may cause a successive filling-out of surface contrast. This and the very short duration of the target $(24 \mathrm{~ms})$ could arise to the impression that the target expands quickly.

Lateral inhibition can also explain the perception of a target integrated inside the mask and the decreasing time course of this perception. Based on lateral inhibition assumptions this integration perception occurs at short SOAs, because at short SOAs the excitatory effect of the target is strong enough not to be to suppress by the inhibitory effect of the mask (Francis, 1997). Therefore, the perception of an integrated target reflects failed masking effects due to the strong representation of the target. As the excitatory effect of the target decreases over time, inhibitory effects of the mask become more effective to suppress the target visibility with increasing SOA. This explains the decreasing time course found for the perception of an integrated target. In addition, Francis (1996) showed that the effect of integration decreases with time, which implies that the failed temporal segregation of two actually separated stimuli decrease over time. These assumptions were incorporated in integration masking models, which suggested a reduction of integration effects and hence target visibility with increasing SOA (Francis \& Cho, 2008; Neumann \& Scharlau, 2007; Reeves, 1982).

To sum up, both perceptions can be explained as an escape of the target processing from lateral inhibition mechanisms at short SOAs. However, as SOA increases, the effect of the lateral inhibition on the target processing inclines, leading to a greater suppression of the target visibility at intermediate SOAs (Francis, 1997; Weisstein, 1968, 1972; Weisstein et al., 1975). This explains the descending time course found for both perceptions.

\subsubsection{High-level masking mechanism at long SOAs}

As described in the beginning of Chapter 6.3.1, it is assumed that a higher-level mechanism is responsible for metacontrast masking effects at long SOAs. However, it remains an open question which higher-level processes can be associated with the two independent perceptions found at long SOAs.

Behavioral evidence. In a first step findings were listed, supporting the assumption that masking effects at long SOAs represent a high-level mechanism. First, the experimental manipulations which affected exclusively the late branch of the metacontrast function cannot be explained solely by lateral inhibition. The metacontrast function at long SOAs was 
influenced by attention manipulations (Bruchmann et al., 2011; Kolers, 1983; Neumann \& Scharlau, 2007; Ramachandran \& Cobb, 1995), figural (Uttal, 1970), semantic similarity of target and mask (Merikle, 1977) or in backward masking paradigms by the semantic of the target (word-nonword) (Michaels \& Turvey, 1979, Experiment E1). The influence of figural or semantic features on the masking function provides evidence that the target needs to be processed and represented not to be disturbed by the mask. This excludes simple feature interferences between target and mask, which could also be explained by lateral inhibition.

Second, evidence for a high-level masking mechanism resulted from common-onset paradigms, in which target and mask appear with simultaneous onset, but delayed offset or four-dot masking paradigms, a special case of common-onset, with masks consisting of four small dots surrounding the target (Di Lollo et al., 2000; Enns \& Di Lollo, 2000). Object Substitution Masking (OSM) was assumed to be a plausible mechanism to explain masking effects in four-dot or common-onset paradigms, where the strength of the contours is far too small for lateral inhibition being the responsible mechanism (Di Lollo et al., 2000; Enns, 2004). OSM was assume to result if the initial and fast processed target information is fed back to lower visual areas after the visual input has changed and instead of the target information, the information of the mask is now processed in detail in a feedforward manner (Di Lollo et al., 2000). As a consequence, the information of the target is substituted by the representation of the mask.

However, the mechanism of object substitution does not seem to be limited exclusively to four-dot masking paradigms. Instead, changes of the masking effects due to attentional manipulations have been found in four-dot masking as well as other types of backward masking paradigms, but only at long ISI (Enns, 2004). Therefore, two distinct visual masking processes were proposed for backward masking. An early process, for example lateral inhibition in the case of metacontrast masking, specific for backward masking paradigms at short SOAs and OSM that underlies both and four-dot and backward masking at long SOAs (Bachmann, 2005; Enns, 2004; Enns \& Di Lollo, 2000).

Nevertheless, it has to be mentioned that metacontrast masking and OSM were also distinguished from each other (e.g. Chakravarthi \& Cavanagh, 2009; Jaśkowski, van der Lubbe, Schlotterbeck, \& Verleger, 2002). Chakravarthi and Cavanagh (2009) assumed that OSM takes place at a higher level of visual processing compared to metacontrast masking, by showing that masking the flankers in a crowing paradigm leads to a recovery of the crowded target, but only for metacontrast masking. The interference of metacontrast masks makes it reasonable to assume that metacontrast masking takes place at an earlier level compare to 
6 | Consideration of metacontrast mechanisms on the basis of phenomenological data

crowding. Since no release from crowding was found for OSM, OSM was classified at a higher processing level than crowding and hence metacontrast masking. However, regarding the very short SOA $(25 \mathrm{~ms})$ between flankers and metacontrast masks, the results of Chakravarthi and Cavanagh (2009) do not contradict with the assumption of this thesis. Instead, at short SOAs a lateral inhibition mechanism is expected for metacontrast masking, which is assume to take place at a lower level of visual processing compare to OSM. Only if the same results were obtained by Chakravarthi and Cavanagh (2009) using longer SOAs for the metacontrast condition, this would provide contra evidence against the assumption that OSM and metacontrast masking at long SOAs share similar mechanisms and processing levels.

Even if the process underlying metacontrast masking at long SOAs can not necessarily be equated with OSM, it is reasonable to assume that they do share properties. These commonalities may help to understand the mechanisms underlying metacontrast masking at the late branch. A common characteristic is the influence of attention manipulation. This, as well as the effect of semantic similarity between target and metacontrast mask, suggests that the representation of target and mask seems to interfere with each other at long SOAs. Consistent with this consideration, the early process underlying short SOAs was associated with a disturbance of the object formation (Enns, 2004), while OSM was explained with an interaction between object representations (Enns, 2004; Hein \& Moore, 2010b). According to this assumption, common-onset masking was also found with a masking object defined by illusory contours, which was interpreted as higher-level object interference, since lower image-level interferences can be ruled out (Hirose \& Osaka, 2009).

Another commonality between both masking mechanisms is that they seem to affect different target features independently. On the one hand, the phenomenological description of OSM, that "the target location appeared empty" (Di Lollo et al., 2000, p. 492) suggests that the target is erased from conscious perception. On the other hand, Enns and Di Lollo (2000) already questioned whether the effect of OSM on a mere detection task, where the target seems to be eliminated by the mask, can be compared with the perception of a specific target feature under OSM. Gellatly et al. (2006) found evidence, that different target features can be affected independently by the mask. He proposed evidence that the dissimilarity or similarity between the stimuli's color and orientation influenced which of the target features was substituted within the representation of the mask. Some authors hypothesized that an influence of OSM on the perception of a target feature depends on whether or not the perception of the features is linked to binding processes (Bouvier \& Treisman, 2010; Koivisto 
\& Silvanto, 2011, 2012). OSM was explained by these authors due to a mismatch within the reentrant loops, combining the slower bottom-up processed information with the top-down projected information. Thus, the perceptions of target features, which involve reentrant processes necessary for feature binding (Treisman, 1996), were found to be affected mostly by OSM. Koivisto and Silvanto (2011) compared four-dot and backward masking effects at different SOAs $(66,106,145 \mathrm{~ms})$. Crosses were used as targets, consisting of a white and a colored bar. Participants had to specify the color and orientation of the colored bar. The color discrimination was not affected, but orientation discrimination was strongly impaired with four-dot masking as well as backward masking at long SOAs. First, this shows that OSM can independently influence different stimulus features. Second, it is consistent with the hypothesis that features that require feature binding are primarily affected, since only orientation discrimination requires feature binding in this paradigm. Third, it provides additional evidence for the similarity of the mechanisms of OSM and those of backward masking at long SOAs. In contrast, at short SOAs even the color perception was reduced, but only under backward masking. This can be interpreted as evidence that another masking mechanism underlies backward masking at short SOAs, compare to long SOAs and OSM. Since no feature binding was necessary to identify the color, this mechanism is probably due to a low-level mechanism.

In accordance, Goodhew (2017) argued that the all-or-none dichotomy in the perception of the target underlying the classical OSM, does not coincide with the existing results. Instead, she proposed to separate the terminology and to speak of an object-updating framework, which includes the possibility that an interaction between target and mask features is reflected in the perception. Even if this differentiation is meaningful, for the sake of clarity the term object substitution continues to be used. Therefore, object substitution describes a masking mechanism in which target features are separately encoded and mistakenly attributed to the mask independently of each other.

Assuming the premise that metacontrast masking at long SOAs is based on a mechanism similar to object substitution, the independence of the two perceptions at long SOAs, a rotation and a target segregated from the mask (Experiment 3), can be explained within an object substitution framework: Since both perceptions reflect the awareness of different target aspects, it should be considered which target information needs to be present to perceive one of the two perceptions. For the perception of a target temporally segregated from the mask at least the surface information of the target needs to be escaped from OSM. Only if it is ensured that the surface information of the target is attributed to the target itself, 
the perception arises that before the presentation of the mask another object was visible. In principle, this could also apply to the shape information of the target, but the definition of perception given in the experiments of this thesis explicitly excludes the need to perceive the target form for the affirmation of a segregated target. Nevertheless, the perception of a temporally segregated target indicates a successful target individuation, at least regarding the surface information.

In contrast, the perception of a rotation implies that the shape information of the target failed individuation. Instead, the information seems to be substituted within the representation of the mask, resulting in the perception of an apparent motion. Since incongruent target-mask pairs were used, the integration of different shape information within the representation of the mask could result in the perception of a rotational movement between target and mask. In accordance, apparent motion in a standing wave illusion paradigm was explained by a failed individuation of the target (Hein \& Moore, 2010b; Pilling \& Gellatly, 2009). Hein and Moore (2010b) assumed that initially built representations were updated over time. If the timing is appropriate and the target information fits well into the representation of the mask both information were integrated. Consequently, differences in the perception associated with independent mechanisms underlying metacontrast masking at long SOAs, can be explained within an object substitution framework by assuming that different target features are substituted independently within the representation of the mask.

With vernier stimuli a dissociation between the time courses of apparent motion and masking was found (Breitmeyer, Herzog, \& Öğmen, 2008), supporting the assumption that the mechanisms underlying the two phenomena were not identical (Breitmeyer \& Horman, 1981; Stoper \& Banffy, 1977; Weisstein \& Growney, 1969). In addition, the motion perception correlated highly with feature attribution, operationalized as attribution of features of the first stimuli to the second stimuli, whereas masking did not (Breitmeyer, Herzog et al., 2008). This speaks in favor for the idea that apparent motion can be explained with the substitution of target features within the representation of the mask, whereas this mechanism seems to be implausible for masking effects, affecting the mere visibility of the target.

The assumption that the features of the target can be substituted independently of each other within the representation of the mask, suits findings that showed latency differences in the processing of different object properties (Lamme \& Roelfsema, 2000; Livingstone \& Hubel, 1988; Zeki, 2015). Zeki (2015) reviewed evidence for the asynchrony of visual object processing inside the time window of around $100 \mathrm{~ms}$, with parallel processing operations of different object features, for example color, form, motion. Also for metacontrast 
masking it has been shown that target's contour or surface feature were processed and masked within different temporal intervals (Bachmann, 2009b; Breitmeyer et al., 2006). Since different target features were assumed to be processed with different time courses, they may prone to being integrated into the representation of the mask at different time point.

To conclude, at long SOAs a high-level masking mechanism, similar to object substitution, was assumed to affect different target features separately. A motion perception was assumed to result from a failed individuation of the target's shape, whereas the individuation of the targets' surface was associated with the perception of a target segregated from the mask.

Electrophysiological evidence. Experiment 4 expanded previous behavioral evidence for different processes associated with phenomenological differences by electrophysiological results. Evidence for two distinct neural signatures accompanying the perceptions of a rotation and a temporally segregated target were found. In this section it should be argued how these electrophysiological results can be explained with an object substitution approach.

Several metacontrast masking theories proposed a disruption of reentrant processes to be responsible for successful masking effects (Bridgeman, 1980; Fahrenfort et al., 2007; Lamme et al., 2002; Tapia \& Breitmeyer, 2011), which has also been incorporated in the object substitution framework (Di Lollo et al., 2000). Also the late latency of both neural signatures implies that differences in the perception may be traced back to recurrent or feedback mechanisms. Feedforward processing was assumed to be reflected by the first two ERPs, the C1 and P1 ( 60-100 ms), whereas the later N1p (140-180 ms) and P2 component (180-220 ms) were associated with top-down feedback mechanisms from higher areas on extrastriate and striate areas (Di Russo et al., 2008). Consequently, Cluster 1, the former of the two neural signatures, which was found at around 230-550 ms after target onset, emerges at a time range which excludes exclusive feedforward processing. The same applies for Cluster 2 found at around 550-840 ms after target onset.

In accordance with the assumption that the rotational apparent motion reflected by Cluster 1 is related to feedback mechanism, evidence for reentrant processing underlying apparent motion was found. With two spatially separated stimuli presented in alternation, apparent motion induced BOLD-fMRI responses were found along the illusory path of apparent motion, receptive fields of early visual areas which were not stimulated by the visual stimulus (Muckli, Kohler, Kriegeskorte, \& Singer, 2005; Sterzer, Haynes, \& Rees, 2006). This was interpreted as a result of top-down feedback from V5/MT+, an area important for motion analysis. In an apparent motion paradigm with concentric rings of different sizes, 
6 | Consideration of metacontrast mechanisms on the basis of phenomenological data

resulting to the impression of an expansion, also evidence for feedback mechanisms responsible for the apparent motion percept were found (Liu, Slotnick, \& Yantis, 2004). Tapia and Beck (2014) reviewed evidence for the necessity of feedback mechanisms from V5/MT+ to $\mathrm{V} 1$ for the perception of an apparent motion.

An interlink between motion perception and object-substitution mechanisms has been provided by Hirose et al. (2007). They showed that OSM was reduced under repetitive transcranial magnetic stimulation (rTMS) applied to V5/MT+. In addition, the motion signal between target and mask, which has been found under OSM, was also lacking with rTMS. This can be interpreted as evidence for the necessity of feedback mechanism for the perception of apparent motion under OSM. Object substitution was explained with an interference of the mask with the target processing not at an earlier time range of object formation, but at a processing level where an interaction between object representations may occur (Enns, 2004; Hein \& Moore, 2010b). The perception of a rotational movement was associated with the failed individuation of the targets shape within the representation of the mask.

The merely perceptual stimulus processing seems to be already completed for the perception of a rotation, which has also been ascribed to earlier components, as the visual awareness negativity (VAN) (Bachmann, 2009a; Railo, Koivisto, \& Revonsuo, 2011; Rutiku et al., 2015). Instead, the already processed perceptual information seems to be updated by higher cognitive mechanisms or hold in working memory, to be bind into one object representation. In accordance, Cluster 1 can be associated with late components, P3 or late positivity (LP), since their latency $(\sim 300-600 \mathrm{~ms}$ after stimulus onset), their large spatial distribution with a maxima at the fronto-parieto network and their direction of effects with higher amplitudes for trials which indicated awareness (Del Cul et al., 2007; Koivisto \& Revonsuo, 2010; Lamy et al., 2008; Sergent et al., 2005), resembles the neural pattern found for Cluster 1. These later components were interpreted as an update of perceptual information by higher cognitive mechanisms, for example attention or working memory, necessary for report (Koivisto \& Revonsuo, 2008; Railo \& Koivisto, 2009; Rutiku et al., 2015; Wilenius \& Revonsuo, 2007) or an identification process of the stimulus at a more central level of information processing (for review see Kok, 1997, 2001). Therefore, these assumptions about the functional significance of Cluster 1 fit well within an object substitution framework.

Woodman and Luck (2003) found electrophysiological evidence that the target information is neural represented under OSM, even if it is not reliably transferred to higher- 
level cognitive processes. Consequently, the neural signature reflecting the awareness of a rotation seem not to occur at an early level of perceptual encoding, instead at a level of higher cognitive re-evaluation of the perceptual information.

The neural signature found for the perception of a rotation was partially generalizable to the perception of a temporally segregated target, whose unique neural signature described an even later process. Thus, even for the perception of a segregated target, parts of the stimulus information seem to fail individuation. The perception of a segregated target was defined only by the perception of the target surface, while the perception of form was explicitly excluded from the definition. Therefore, the individuation of the form information could also fail individuation for the perception of a segregated target. Since an unnoticed failure of individuation cannot be excluded, this can serve as an explanation for the partial generalizability of Cluster 1 .

Based on behavioral results it was assumed that the perception of a rotation can be equated with a failed individuation of the targets shape information. Instead, the perception of a temporally segregated target was explained with an escape of surface information from being substituted within the representation of the mask. In other words, the perception of a rotation rather reflects a successful masking whereas the perception of a segregated target seems to represent a failed masking at least of the targets shape. These considerations are in line with the later processing stage of Cluster 2, compared to the cluster representing a rotation. The later processing stage can be interpreted as evidence that the perception of a segregate target is accompanied by a more complete processing of the target's information, compared to the perception of a rotation. Based on the late latency and the higher amplitudes for trials which indicated no awareness, it has been associated with slow negativity wave, which may index central resources, like visual short-term memory process (Mecklinger \& Pfeifer, 1996; Ruchkin et al., 1995; Vogel \& Machizawa, 2004). The higher memory load can be explained by the need to store the perception of the target, the mask and their temporal relationship to decide whether or not a temporally segregated target has been perceived. But based on the present results this supposition must be considered with caution. Though, based on the late latency this neural signature seems rather reflect a deficit in central resources, as working memory, than any differences in perceptual processing (Kok, 1997). 


\subsection{Concluding Remarks}

Evidence for a rich and detailed perception, even under conditions of reduced visibility as metacontrast masking has been provided (Experiment 1 - 2). Despite the usage of simple, low-level stimuli and the high difficulty of perceiving the very subtle perceptual variations, six categories could be validated as reliable perceptual dimensions of target perception at metacontrast masking. Since these perceptual categories showed gradual variations in dependence of the parametric changes, it can be concluded that these perceptions are not merely a result of trial-by-trial fluctuations in the perceptual capabilities. The agreement between the results of Experiment 1-3 regarding the time course of the perceptions highlights that this phenomenological investigation was able to overcome problems of earlier introspective investigations (Velmans, 2007; Vermersch, 1999). In accordance, this refutes the criticism that introspection is illusory or even delusional (Haun et al., 2017).

The richness of the target perception as evident by its diverse phenomenology is in contrast with the low discrimination performance found at long SOAs (Experiment 2). It supports the claim that simple, low-level phenomenological categories capture the complexity of the perception more adequately than predetermined high-level categories (Haun et al., 2017). This shows the importance to define the criterion content participants should base their performance on to yield consistent metacontrast functions. The current data emphasize that a seemingly unambiguous objective task, for example to identify the target, can be performed in very different ways, depending on the individual ability to spontaneously perceive and use the different target aspects arising at different conditions.

Further, evidence for three different processes underlying metacontrast masking was provided, each of them related with a distinct perception (Experiment 3). In accordance, electrophysiological results supported the assumption about different processes underlying metacontrast at long SOAs, since the neural signature of processing the target differ in dependence on the perception.

Regarding the phenomenology and the evidence for independent processes it seems to be inappropriate to conceptualize metacontrast masking with a unitary process underlying the whole range of SOAs. Instead, metacontrast can be defined as multidimensional phenomenon with three different processes underlying short and long SOAs. Considering metacontrast masking as a multidimensional phenomenon challenges the usage of one-dimensional subjective or objective measures to capture the perception of the target. Qualitative differences in the appearance of the target could go unnoticed with one-dimensional 
measures. This indicates the superiority of qualitative subjective measures compare to onedimensional objective measures, if the aim is to capture as much awareness of the target as possible. Furthermore, without considering the richness and diversity of the target perception in a metacontrast paradigm, statements about underlying processes may be subject to error. Evidence for distinct neural signatures depending on the target perception challenges approaches to identify NCC by contrasting conditions in a metacontrast paradigm without considering differences in the phenomenology. The subtraction of ERPs of different conditions to control for differences in the physical stimulation seems to be inappropriate for metacontrast masking. This procedure is based on two assumptions. First, that the neuronal processing of the target is only additively influenced by the mask and second, that it differs only quantitatively between different SOAs. On the one hand, however, this thesis provided evidence that both temporal and spatial variations qualitatively influence the perception of the target. On the other hand, it was found that neuronal processing differs depending on perception. Therefore, the additive assumption of metacontrast seems to be untenable for metacontrast masking and investigations using this method may be subject to error. Further, the variability in the neural processing based on differences in the appearance also challenges approaches to identify a uniform NCC for metacontrast masking. Instead, neural correlates, which index the awareness in a metacontrast masking paradigm, appear to be as diverse as the perception of the target in different conditions.

At the present time, it is unclear whether the criticisms applies only to metacontrast masking paradigms or if it can be generalized to other types of masking. The short literature review of Section 6.2.2 at least points out that caution is required, since qualitative differences in experience also occur in other types of masking. 


\section{References}

Ahissar, M., \& Hochstein, S. (1997). Task difficulty and the specificity of perceptual learning. Nature, 387, 401-406.

Ahissar, M., \& Hochstein, S. (2004). The reverse hierarchy theory of visual perceptual learning. Trends in Cognitive Sciences, 8(10), 457-464.

https://doi.org/10.1016/j.tics.2004.08.011

Ahissar, M., Nahum, M., Nelken, I., \& Hochstein, S. (2009). Reverse hierarchies and sensory learning. Philosophical Transactions of the Royal Society B: Biological Sciences, 364(1515), 285-299. https://doi.org/10.1098/rstb.2008.0253

Albrecht, T., Klapötke, S., \& Mattler, U. (2010). Individual differences in metacontrast masking are enhanced by perceptual learning. Consciousness and Cognition, 19(2), 656666. https://doi.org/10.1016/j.concog.2009.12.002

Albrecht, T., \& Mattler, U. (2012a). Individual differences in subjective experience and objective performance in metacontrast masking. Journal of Vision, 12(5), 5. https://doi.org/10.1167/12.5.5

Albrecht, T., \& Mattler, U. (2012b). Individual differences in metacontrast masking regarding sensitivity and response bias. Consciousness and Cognition, 21(3), 1222-1231. https://doi.org/10.1016/j.concog.2012.04.006

Albrecht, T., \& Mattler, U. (2016). Individually different weighting of multiple processes underlies effects of metacontrast masking. Consciousness and Cognition, 42, 162-180. https://doi.org/10.1016/j.concog.2016.03.006

Alpern, M. (1953). Metacontrast. Journal of the Optical Society of America, 43(8).

Ansorge, U., Becker, S. I., \& Breitmeyer, B. G. (2009). Revisiting the metacontrast dissociation: Comparing sensitivity across different measures and tasks. The Quarterly Journal of Experimental Psychology, 62(2), 286-309. https://doi.org/10.1080/17470210801908492

Ansorge, U., Breitmeyer, B. G., \& Becker, S. I. (2007). Comparing sensitivity across different processing measures under metacontrast masking conditions. Vision Research, 47(27), 3335-3349. https://doi.org/10.1016/j.visres.2007.09.009

Anstis, S. M., \& Mather, G. (1985). Effects of luminance and contrast on direction of ambiguous apparent motion. Perception, 14(2), 167-179. https://doi.org/10.1068/p140167 
Arrington, K. F. (1994). The temporal dynamics of brightness filling-in. Vision Research, 34(24), 3371-3387. https://doi.org/10.1016/0042-6989(94)90071-X

Baayen, R. H., Davidson, D. J., \& Bates, D. M. (2008). Mixed-effects modeling with crossed random effects for subjects and items. Journal of Memory and Language, 59(4), 390-412. https://doi.org/10.1016/j.jml.2007.12.005

Bachmann, T. (1988). Time course of the subjective contrast enhancement for a second stimulus in successively paired above-threshold transient forms: Perceptual retouch instead of forward masking. Vision Research, 28(11), 1255-1261.

Bachmann, T. (2005). Object substitution and its relation to other forms of visual masking: Reply to James Enns. Vision Research, 45(3), 381-385. https://doi.org/10.1016/j.visres.2004.08.011

Bachmann, T. (2009a). Finding ERP-signatures of target awareness: Puzzle persists because of experimental co-variation of the objective and subjective variables. Consciousness and Cognition, 18(3), 804-808. https://doi.org/10.1016/j.concog.2009.02.011

Bachmann, T. (2009b). Metacontrast masking of target-area internal contours and target overall surface brightness: The case of mutually coherent and incoherent visual objects. Spatial Vision, 22(2), 127-146.

Bachmann, T. (2015). Unmasking the pitfalls of the masking method in consciousness research. In M. Overgaard (Ed.), Behavioral Methods in Consciousness Research (pp. 4975). Oxford University Press.

Bachmann, T., \& Francis, G. (2003). Visual masking: Studying perception, attention and consciousness: Elsevier.

Bachmann, T., \& Kirt, T. (2013). Perception of successive brief objects as a function of stimulus onset asynchrony: Model experiments based on two-stage synchronization of neuronal oscillators. Cognitive Neurodynamics, 7(6), 465-475. https://doi.org/10.1007/s11571-013-9250-4

Bar, M. (2003). A cortical mechanism for triggering top-down facilitation in visual object recognition. Journal of Cognitive Neuroscience, 15(4), 600-609. https://doi.org/10.1162/089892903321662976

Bates, D., Mächler, M., Bolker, B., \& Walker, S. (2015). Fitting linear mixed-effects models using lme4. Journal of Statistical Software, 67(1), 1-48.

https://doi.org/10.18637/jss.v067.i01 
Beck, J., Elsner, A., \& Silverstein, C. (1977). Position uncertainty and the perception of apparent movement. Perception \& Psychophysics, 21(1), 33-38. https://doi.org/10.3758/BF03199464

Becker, M. W., \& Anstis, S. (2004). Metacontrast masking is specific to luminance polarity. Vision Research, 44(21), 2537-2543. https://doi.org/10.1016/j.visres.2004.05.007

Bouvier, S., \& Treisman, A. (2010). Visual feature binding requires reentry. Psychological Science, 21(2), 200-204. https://doi.org/10.1177/0956797609357858

Breitmeyer, B. G. (1978a). Metacontrast masking as a function of mask energy. Bulletin of the Psychonomic Society, 12(1), 50-52. https://doi.org/10.3758/BF03329621

Breitmeyer, B. G. (1978b). Metacontrast with black and white stimuli: Evidence for inhibition of on- and off-sustained activity by either on- or off-transient activity. Vision Research, 18, $1443-1448$.

Breitmeyer, B. G. (2014). Contributions of magno- and parvocellular channels to conscious and non-conscious vision. Philosophical Transactions of the Royal Society B: Biological Sciences, 369, 1-11. https://doi.org/10.1098/rstb.2013.0213

Breitmeyer, B. G., Battaglia, F., \& Weber, C. (1976). 'U'-shaped backward contour masking during stroboscopic motion. Journal of Experimental Psychology: Human Perception and Performance, 2(2), 167-173. https://doi.org/10.1037/0096-1523.2.2.167

Breitmeyer, B. G., \& Ganz, L. (1976). Implications of sustained and transient channels for theories of visual pattern masking, saccadic suppression, and information processing. Psychological Review, 83(1), 1-36. https://doi.org/10.1037/0033-295X.83.1.1

Breitmeyer, B. G., Herzog, M. H., \& Öğmen, H. (2008). Motion, not masking, provides the medium for feature attribution. Psychonomic Science, 19(8), 823-829.

Breitmeyer, B. G., \& Horman, K. (1981). On the role of stroboscopic motion in metacontrast. Bulletin of the Psychonomic Society, 17(1), 29-32. https://doi.org/10.3758/BF03333658

Breitmeyer, B. G., \& Jacob, J. (2012). Microgenesis of surface completion in visual objects: Evidence for filling-out. Vision Research, 55, 11-18. https://doi.org/10.1016/j.visres.2011.12.010

Breitmeyer, B. G., Kafaligonul, H., Öğmen, H., Mardon, L., Todd, S. J., \& Ziegler, R. (2006). Meta- and paracontrast reveal differences between contour- and brightness-processing mechanisms. Vision Research, 46(17), 2645-2658. https://doi.org/10.1016/j.visres.2005.10.020 
Breitmeyer, B. G., Love, R., \& Wepman, B. (1974). Contour suppression during stroboscopic motion and metacontrast. Vision Research, 14(12), 1451-1456.

https://doi.org/10.1016/0042-6989(74)90020-0

Breitmeyer, B. G., \& Öğmen, H. (2006). Visual masking: Time slices through conscious and unconscious vision. Oxford: University Press.

Breitmeyer, B. G., Tapia, E., Kafaligonul, H., \& Öğmen, H. (2008). Metacontrast masking and stimulus contrast polarity. Vision Research, 48(23-24), 2433-2438.

https://doi.org/10.1016/j.visres.2008.08.003

Bridgeman, B. (1971). Metacontrast and lateral inhibition. Psychological Review, 78(6), 528539. https://doi.org/10.1037/h0031782

Bridgeman, B. (1980). Temporal response characteristics of cells in monkey striate cortex measured with metacontrast masking and brightness discrimination. Brain Research, 196(2), 347-364. https://doi.org/10.1016/0006-8993(80)90400-X

Bridgeman, B. (2001). A comparison of two lateral inhibitory models of metacontrast. Journal of Mathematical Psychology, 45(5), 780-788.

https://doi.org/10.1006/jmps.2000.1352

Bruchmann, M., Hintze, P., \& Mota, S. (2011). The effects of spatial and temporal cueing on metacontrast masking. Advances in Cognitive Psychology, 7, 132-141. https://doi.org/10.2478/v10053-008-0093-1

Brussell, E. M., Stober, S. R., \& Favreau, O. E. (1978). Contrast reversals in backward masking. Vision Research, 18(2), 225-227.

Chakravarthi, R., \& Cavanagh, P. (2009). Recovery of a crowded object by masking the flankers: Determining the locus of feature integration. Journal of Vision, 9(10), 1-9. https://doi.org/10.1167/9.10.4

Chalmers, D. J. (1995). Facing up to the problem of consciousness. Journal of Consciousness Studies, 2(3), 200-219.

Chung, S. T.L. (2007). Learning to identify crowded letters: Does it improve reading speed? Vision Research, 47(25), 3150-3159.

Chung, S. T.L., Legge, G. E., \& Cheung, S.-h. (2004). Letter-recognition and reading speed in peripheral vision benefit from perceptual learning. Vision Research, 44(7), 695-709. https://doi.org/10.1016/j.visres.2003.09.028 
Cohen, J. (1960). A coefficient of agreement for nominal scales. Educational and Psychological Measurement, 20, 37-46.

Cohen, M. A., Dennett, D. C., \& Kanwisher, N. (2016). What is the Bandwidth of Perceptual Experience? Trends in Cognitive Sciences, 20(5), 324-335. https://doi.org/10.1016/j.tics.2016.03.006

Costall, A. (2006). 'Introspectionism' and the mythical origins of scientific psychology. Consciousness and Cognition, 15(4), 634-654. https://doi.org/10.1016/j.concog.2006.09.008

Del Cul, A., Baillet, S., \& Dehaene, S. (2007). Brain dynamics underlying the nonlinear threshold for access to consciousness. PLoS Bio, 5(10). https://doi.org/10.1371/journal.pbio.0050260.g001

Delorme, A., \& Makeig, S. (2004). EEGLAB: An open toolbox for analysis of single-trial EEG dynamics including independent component analysis. Journal of Neuroscience Methods, 134, 9-21.

Dennett, D. C. (1991). Consciousness explained. Boston: Little, Brown and Co.

Dennett, D. C. (2003). Who's On First? Heterophenomenology Explained. Journal of Consciousness Studies, 10(9-10), 10-30.

Dennett, D. C. (2007). Heterophenomenology reconsidered. Phenomenology and the Cognitive Sciences, 6, 247-270. https://doi.org/10.1007/s11097-006-9044-9

Di Lollo, V., Enns, J. T., \& Rensink, R. A. (2000). Competition for consciousness among visual events: The psychophysics of reentrant visual processes. Journal of Experimental Psychology: General, 129(5), 481-507.

Di Russo, F., Aprile, T., Spitoni, G., \& Spinelli, D. (2008). Impaired visual processing of contralesional stimuli in neglect patients: A visual-evoked potential study. Brain, 131, 842-854. https://doi.org/10.1093/brain/awm281

Dienes, Z., \& Seth, A. (2010). Gambling on the unconscious: A comparison of wagering and confidence ratings as measures of awareness in an artificial grammar task. Consciousness and Cognition, 19(2), 674-681. https://doi.org/10.1016/j.concog.2009.09.009

Dombrowe, I., Hermens, F., Francis, G., \& Herzog, M. H. (2009). The roles of mask luminance and perceptual grouping in visual backward masking. Journal of Vision, 9(11), 22. https://doi.org/10.1167/9.11.22 
Drewes, J., Zhu, W., \& Melcher, D. (2014). Dissociation between spatial and temporal integration mechanisms in vernier fusion. Vision Research, 105, 21-28. https://doi.org/10.1016/j.visres.2014.08.017

Duangudom, V., Francis, G., \& Herzog, M. H. (2007). What is the strength of a mask in visual metacontrast masking? Journal of Vision, 7(1), 7. https://doi.org/10.1167/7.1.7

Eimer, M., \& Mazza, V. (2005). Electrophysiological correlates of change detection. Psychophysiology, 42(3), 328-342. https://doi.org/10.1111/j.1469-8986.2005.00285.x

Enns, J. T. (2002). Visual binding in the standing wave illusion. Psychonomic Bulletin \& Review, 9(3), 489-496. https://doi.org/10.3758/BF03196304

Enns, J. T. (2004). Object substitution and its relation to other forms of visual masking. Vision Research, 44(12), 1321-1331. https://doi.org/10.1016/j.visres.2003.10.024

Enns, J. T., \& Di Lollo, V. (2000). What's new in visual masking? Trends in Cognitive Sciences, 4(9), 345-352. https://doi.org/10.1016/S1364-6613(00)01520-5

Eriksen, C. W., \& Rohrbauch, J. (1970). Visual masking in multielement displays. Journal of Experimental Psychology: General, 83(1), 147-154.

Fahrenfort, J. J., Scholte, H. S., \& Lamme, V. A. F. (2007). Masking disrupts reentrant processing in human visual cortex. Journal of Cognitive Neuroscience, 19(9), 1488-1497. https://doi.org/10.1162/jocn.2007.19.9.1488

Fehrer, E., \& Biederman, I. (1962). A comparison of reaction time and verbal report in the detection of masked stimuli. Journal of Experimental Psychology, 64(2), 126-130. https://doi.org/10.1037/h0044410

Fehrer, E., \& Raab, D. (1962). Reaction time to stimuli masked by metacontrast. Journal of Experimental Psychology, 63(2), 143-147. https://doi.org/10.1037/h0040795

Fei-Fei, L., Iyer, A., Koch, C., \& Perona, P. (2007). What do we perceive in a glance of a real-world scene? Journal of Vision, 7(1), 10. https://doi.org/10.1167/7.1.10

Fenske, M. J., Aminoff, E., Gronau, N., \& Bar, M. (2006). Top-down facilitation of visual object recognition: Object-based and context-based contributions. In S. Martinez-Conde, S. L. Macknik, L. M. Martinez, J.-M. Alonso, \& P. U. Tse (Eds.), Progress in Brain Research. Elsevier.

Fleischhauer, M., Miller, R., Enge, S., \& Albrecht, T. (2014). Need for cognition relates to low-level visual performance in a metacontrast masking paradigm. Journal of Research in Personality, 48, 45-50. https://doi.org/10.1016/j.jrp.2013.09.007 
Francis, G. (1996). Cortical dynamics of visual persistence and temporal integration. Perception \& Psychophysics, 58(8), 1203-1212. https://doi.org/10.3758/BF03207553

Francis, G. (1997). Cortical dynamics of lateral inhibition: Metacontrast masking. Psychological Review, 104(3), 572-594.

Francis, G. (2007). What should a quantitative model of masking look like and why would we want it? Advances in Cognitive Psychology, 3(1-2), 21-31. https://doi.org/10.2478/v10053008-0011-6

Francis, G., \& Cho, Y. S. (2008). Effects of temporal integration on the shape of visual backward masking functions. Journal of Experimental Psychology: Human Perception and Performance, 34(5), 1116-1128. https://doi.org/10.1037/0096-1523.34.5.1116

Gallagher, S. (2003). Phenomenology and experimental design: Towards a phenomenologically enlightened experimental science. Journal of Consciousness Studies, 10(9-10), 85-99.

Gallagher, S., \& Sørensen, J. B. (2006). Experimenting with phenomenology. Consciousness and Cognition, 15(1), 119-134. https://doi.org/10.1016/j.concog.2005.03.002

Gallagher, S., \& Varela, F. J. (2003). Redrawing the map and resetting the time: Phenomenology and the cognitive sciences. Canadian Journal of Philosophy, 33, 93-132. https://doi.org/10.1080/00455091.2003.10717596

Gallagher, S., \& Zahavi, D. (2008). The Phenomenological Mind: An Introduction to Philosophy of Mind and Cognitive Science. New York: Routledge.

Gellatly, A., Pilling, M., Cole, G., \& Skarratt, P. (2006). What is being masked in object substitution masking? Journal of Experimental Psychology: Human Perception and Performance, 32(6), 1422-1435. https://doi.org/10.1037/0096-1523.32.6.1422

Goodhew, S. C. (2017). What have we learned from two decades of object-substitution masking? Time to update: Object individuation prevails over substitution. Journal of Experimental Psychology: Human Perception and Performance, 43(6), 1249-1262. https://doi.org/10.1037/xhp0000395

Growney, R. L., Weisstein, N., \& Cox, S. I. (1977). Metacontrast as a function of spatial separation with narrow line targets and masks. Vision Research, 17(10), 1205-1210. https://doi.org/10.1016/0042-6989(77)90155-9 
Haun, A. M., Tononi, G., Koch, C., \& Tsuchiya, N. (2017). Are we underestimating the richness of visual experience? Neuroscience of Consciousness, 3(1). https://doi.org/10.1093/nc/niw023

Hauntus, M. J. (1995). Corrections for extreme proportions and their biasing effects on estimated values of d'. Behavior Research Methods, Instruments, \& Computers, 27(1), 4651.

Heckenmueller, E. G., \& Dember, W. N. (1965a). A forced-choice indicator for use with Werner's disc-ring pattern in studies of backward masking. Psychonomic Science, 3(1-12), 167-168. https://doi.org/10.3758/BF03343076

Heckenmueller, E. G., \& Dember, W. N. (1965b). Paradoxical brightening of a masked black disc. Psychonomic Science, 3(1-12), 457-458. https://doi.org/10.3758/BF03343230

Hein, E., \& Moore, C. M. (2010a). Lateral masking in cycling displays: The relative importance of separation, flanker duration, and interstimulus interval for object-mediated updating. Perception, 39, 1330-1340.

Hein, E., \& Moore, C. M. (2010b). Unmasking the standing wave of invisibility: An account in terms of object-mediated representational updating. Attention, Perception, \& Psychophysics, 72(2), 398-408.

Herzog, M. H., Fahle, M., \& Koch, C. (2001). Spatial aspects of object formation revealed by a new illusion, shine-through. Vision Research, 41(18), 2325-2335. https://doi.org/10.1016/S0042-6989(01)00122-5

Herzog, M. H., Harms, M., Ernst, U. A., Eurich, C. W., Mahmud, S. H., \& Fahle, M. (2003). Extending the shine-through effect to classical masking paradigms. Vision Research, 43(25), 2659-2667. https://doi.org/10.1016/S0042-6989(03)00461-9

Herzog, M. H., Lesemann, E., \& Eurich, C. W. (2006). Spatial interactions determine temporal feature integration as revealed by unmasking. Advances in Cognitive Psychology, 2(1), 77-85. https://doi.org/10.2478/v10053-008-0046-8

Herzog, M. H., Schmonsees, U., \& Fahle, M. (2003). Timing of contextual modulation in the shine-through effect. Vision Research, 43(19), 2039-2051. https://doi.org/10.1016/S00426989(03)00313-4

Hirose, N., Kihara, K., Mima, T., Ueki, Y., Fukuyama, H., \& Osaka, N. (2007). Recovery from object substitution masking induced by transient suppression of visual motion processing: A repetitive transcranial magnetic stimulation study. Journal of Experimental 
Psychology: Human Perception and Performance, 33(6), 1495-1503.

https://doi.org/10.1037/0096-1523.33.6.1495

Hirose, N., \& Osaka, N. (2009). Object substitution masking induced by illusory masks:

Evidence for higher object-level locus of interference. Journal of Experimental

Psychology: Human Perception and Performance, 35(4), 931-938.

https://doi.org/10.1037/a0012734

Hogben, J. H., \& Di Lollo, V. (1984). Practice reduces suppression in metacontrast and in apparent motion. Perception \& Psychophysics, 35(5), 441-445.

https://doi.org/10.3758/BF03203920

Hubel, D. H., \& Wiesel, T. N. (1962). Receptive fields, binocular interaction and functional architecture in the cat's visual cortex. The Journal of Physiology, 160(1), 106-154. https://doi.org/10.1113/jphysiol.1962.sp006837

Irwin, D. E. (1991). Information integration across saccadic eye movements. Cognitive Psychology, 23, 420-456.

Ishikawa, A., Shimegi, S., \& Sato, H. (2006). Metacontrast masking suggests interaction between visual pathways with different spatial and temporal properties. Vision Research, 46(13), 2130-2138. https://doi.org/10.1016/j.visres.2005.12.013

James, W. (1890a). The Principles of Psychology (Vol I). London: Holt.

James, W. (1890b). The Principles of Psychology (Vol II). London: Holt.

Jannati, A., \& Di Lollo, V. (2012). Relative blindsight arises from a criterion confound in metacontrast masking: Implications for theories of consciousness. Consciousness and Cognition, 21(1), 307-314. https://doi.org/10.1016/j.concog.2011.10.003

Jaśkowski, P., van der Lubbe, R., Schlotterbeck, E., \& Verleger, R. (2002). Traces left on visual selective attention by stimuli that are not consciously identified. Psychological Science, 13(1), 48-54.

Kahneman, D. (1967). An onset-onset law for one case of apparent motion and metacontrast. Perception \& Psychophysics, 2(12), 577-584. https://doi.org/10.3758/BF03210272

Kahneman, D. (1968). Method, findings, and theory in studies of visual masking. Psychological Bulletin, 70(6), 404-425.

Kanwisher, N. (2001). Neural events and perceptual awareness. Cognition, 79(1-2), 89-113. https://doi.org/10.1016/S0010-0277(00)00125-6 
Kirt, T., \& Bachmann, T. (2013). Perceptual retouch theory derived modeling of interactions in the processing of successive visual objects for consciousness: Two-stage synchronization of neuronal oscillators. Consciousness and Cognition, 22(1), 330-347. https://doi.org/10.1016/j.concog.2012.07.007

Klaver, P., Talsma, D., Wijers, A. A., Heinze, H.-J., \& Mulder, G. (1999). An event-related brain potential correlate of visual short-term memory. NeuroReport, 10, 2001-2005.

Koch, C., Massimini, M., Boly, M., \& Tononi, G. (2016). Neural correlates of consciousness: Progress and problems. Nature Reviews Neuroscience, 17(5), 307-321. https://doi.org/10.1038/nrn.2016.22

Koivisto, M., \& Grassini, S. (2016). Neural processing around $200 \mathrm{~ms}$ after stimulus-onset correlates with subjective visual awareness. Neuropsychologia, 84, 235-243. https://doi.org/10.1016/j.neuropsychologia.2016.02.024

Koivisto, M., \& Revonsuo, A. (2008). The role of selective attention in visual awareness of stimulus features: Electrophysiological studies. Cognitive, Affective, \& Behavioral Neuroscience, 8(2), 195-210. https://doi.org/10.3758/CABN.8.2.195

Koivisto, M., \& Revonsuo, A. (2010). Event-related brain potential correlates of visual awareness. Neuroscience and Biobehavioral Reviews, 34(6), 922-934. https://doi.org/10.1016/j.neubiorev.2009.12.002

Koivisto, M., Revonsuo, A., \& Lehtonen, M. (2006). Independence of visual awareness from the scope of attention: An electrophysiological study. Cerebral Cortex, 16(3), 415-424. https://doi.org/10.1093/cercor/bhi121

Koivisto, M., \& Silvanto, J. (2011). Relationship between visual binding, reentry and awareness. Consciousness and Cognition, 20(4), 1293-1303. https://doi.org/10.1016/j.concog.2011.02.008

Koivisto, M., \& Silvanto, J. (2012). Visual feature binding: The critical time windows of V1/V2 and parietal activity. NeuroImage, 59(2), 1608-1614. https://doi.org/10.1016/j.neuroimage.2011.08.089

Kok, A. (1997). Event-related-potential (ERP) reflections of mental resources: A review and synthesis. Biological Psychology, 45, 19-56.

Kok, A. (2001). On the utility of P3 amplitude as a measure of processing capacity. Psychophysiology, 38(3), 557-577. https://doi.org/10.1017/S0048577201990559 
Kolers, P. A. (1983). Perception and representation. Annual Review of Psychology, 34(1), $129-166$.

Kolers, P. A., \& Rosner, B. S. (1960). On visual masking (metacontrast): Dichoptic observation. The American Journal of Psychology, 73(1), 2-21.

Kouider, S., Gardelle, V. de, Sackur, J., \& Dupoux, E. (2010). How rich is consciousness? The partial awareness hypothesis. Trends in Cognitive Sciences, 14(7), 301-307. https://doi.org/10.1016/j.tics.2010.04.006

Lamme, V. A. F., \& Roelfsema, P. R. (2000). The distinct modes of vision offered by feedforward and recurrent processing. Trends in Neurosciences, 23(11), 571-579. https://doi.org/10.1016/S0166-2236(00)01657-X

Lamme, V. A. F., Zipser, K., \& Spekreijse, H. (2002). Masking interrupts figure-ground signals in V1. Journal of Cognitive Neuroscience, 14(7), 1044-1053.

Lamy, D., Salti, M., \& Bar-Haim, Y. (2008). Neural correlates of subjective awareness and unconscious processing: An ERP study. Journal of Cognitive Neuroscience, 21(7), 14351446.

Lau, H. C., \& Passingham, R. E. (2006). Relative blindsight in normal observers and the neural correlate of visual consciousness. Proceedings of the National Academy of Sciences, 103(49), 18763-18768. https://doi.org/10.1073/pnas.0607716103

Lefton, L. A., \& Newman, Y. (1976). Metacontrast and paracontrast: Both photopic and scotopic luminance levels yield monotones. Bulletin of the Psychonomic Society, 8(6), $435-438$.

Liu, T., Slotnick, S. D., \& Yantis, S. (2004). Human MT+ mediates perceptual filling-in during apparent motion. NeuroImage, 21(4), 1772-1780. https://doi.org/10.1016/j.neuroimage.2003.12.025

Livingstone, M., \& Hubel, D. (1988). Segregation of form, color, movement, and depth: Anatomy, physiology, and perception. Science, 240, 740-749. https://doi.org/10.1126/science.3283936

Lleras, A., \& Moore, C. M. (2003). When the target becomes the mask: Using apparent motion to isolate the object-level component of object substitution masking. Journal of Experimental Psychology: Human Perception and Performance, 29(1), 106-120. https://doi.org/10.1037/0096-1523.29.1.106 
Luck, S. J. (2005). An introduction to the event-related potential technique (First edition). Cambridge: The MIT Press.

Lutz, A., Lachaux, J.-P., Martinerie, J., \& Varela, F. J. (2002). Guiding the study of brain dynamics by using firstperson data: Synchrony patterns correlate with ongoing conscious states during a simple visual task. Proceedings of the National Academy of Sciences, 99(3), $1586-1591$.

Macknik, S. L., \& Livingstone, M. S. (1998). Neuronal correlates of visibility and invisibility in the primate visual system. Nature, 1(2), 144-149.

Macknik, S. L., \& Martinez-Conde, S. (2004). Dichoptic visual masking reveals that early binocular neurons exhibit weak interocular suppression: Implications for binocular vision and visual awareness. Journal of Cognitive Neuroscience, 16(6), 1049-1059.

Macknik, S. L., \& Martinez-Conde, S. (2007). The role of feedback in visual masking and visual processing. Advances in Cognitive Psychology, 3(1-2), 125-152. https://doi.org/10.2478/v10053-008-0020-5

Macmillan, N. A., \& Creelman, C. D. (1991). Detection theory: A user's guide. Cambrige: Cambridge University Press.

Maksimov, M., Murd, C., \& Bachmann, T. (2011). Target-mask shape congruence impacts the type of metacontrast masking. Scandinavian Journal of Psychology, 52(6), 524-529. https://doi.org/10.1111/j.1467-9450.2011.00904.x

Maris, E., \& Oostenveld, R. (2007). Nonparametric statistical testing of EEG- and MEG-data. Journal of Neuroscience Methods, 164(1), 177-190. https://doi.org/10.1016/j.jneumeth.2007.03.024

Mecklinger, A., \& Pfeifer, E. (1996). Event-related potentials reveal topographical and temporal distinct neuronal activation patterns for spatial and object working memory. Cognitive Brain Research, 4(3), 211-224. https://doi.org/10.1016/S0926-6410(96)00034-1

Merikle, P. M. (1977). On the nature of metacontrast with complex targets and masks. Journal of Experimental Psychology: Human Perception and Performance, 3(4), 607-621. https://doi.org/10.1037/0096-1523.3.4.607

Merikle, P. M. (1992). Perception without awareness: Critical issues. American Psychologist, 47(6), 792-795. https://doi.org/10.1037//0003-066X.47.6.792

Michaels, C., \& Turvey, M. T. (1979). Central sources of visual masking: Indexing structures supporting seeing at a single, brief glance. Psychological Review, 41, 1-61. 
Moore, C. M., \& Lleras, A. (2005). On the role of object representations in substitution masking. Journal of Experimental Psychology: Human Perception and Performance, 31(6), 1171-1180. https://doi.org/10.1037/0096-1523.31.6.1171

Muckli, L., Kohler, A., Kriegeskorte, N., \& Singer, W. (2005). Primary visual cortex activity along the apparent-motion trace reflects illusory perception. PLoS Biology, 3(8), e265. https://doi.org/10.1371/journal.pbio.0030265

Nagel, T. (1974). What is it like to be a bat? The Philosophical Review, 83(4), 435-450.

Neumann, O., \& Scharlau, I. (2007). Visual attention and the mechanism of metacontrast. Psychological Research, 71(6), 626-633. https://doi.org/10.1007/s00426-006-0061-7

Nisbett, R. E., \& Wilson, T. D. (1977). Telling more than we can know: Verbal reports on mental processes. Psychological Review, 84(3), 231-259.

Öğmen, H., Breitmeyer, B. G., \& Melvin, R. (2003). The what and where in visual masking. Vision Research, 43(12), 1337-1350. https://doi.org/10.1016/S0042-6989(03)00138-X

Orban, G. A. (2008). Higher order visual processing in macaque extrastriate cortex. Physiological Reviews, 88(1), 59-89. https://doi.org/10.1152/physrev.00008.2007

O'Regan, J. K., \& Noë, A. (2001). A sensorimotor account of vision and visual consciousness. Behavioral and Brain Sciences, 24(05), 939-973. https://doi.org/10.1017/S0140525X01000115

Overgaard, M. (2004). On the naturalising of phenomenology. Phenomenology and the Cognitive Sciences, 3, 365-379.

Overgaard, M. (2006). Introspection in science. Consciousness and Cognition, 15(4), 629633. https://doi.org/10.1016/j.concog.2006.10.004

Overgaard, M., \& Fazekas, P. (2016). Can no-report paradigms extract true correlates of consciousness? Trends in Cognitive Sciences, 20(4), 241-242. https://doi.org/10.1016/j.tics.2016.01.004

Overgaard, M., Jensen, M., \& Sandberg, K. (2009). Methodological pitfalls in the "objective" approach to consciousness: Comments on Busch et al. (2009). Journal of Cognitive Neuroscience, 22(9), 1901-1902.

Overgaard, M., Rote, J., Mouridsen, K., \& Ramsøy, T. Z. (2006). Is conscious perception gradual or dichotomous? A comparison of report methodologies during a visual task. Consciousness and Cognition, 15(4), 700-708. https://doi.org/10.1016/j.concog.2006.04.002 
Panis, S., \& Schmidt, T. (2016). What is shaping RT and accuracy distributions? Active and selective response inhibition causes the negative compatibility Effect. Journal of Cognitive Neuroscience, 28(11), 1651-1671.https://doi.org/10.1162/jocn_a_00998

Paradiso, M. A., \& Nakayama, K. (1991). Brightness perception and filling-in. Vision Research, 31(7-8), 1221-1236. https://doi.org/10.1016/0042-6989(91)90047-9

Persaud, N., McLeod, P., \& Cowey, A. (2007). Post-decision wagering objectively measures awareness. Nature Neuroscience, 10(2), 257-261. https://doi.org/10.1038/nn1840

Petry, S. (1978). Perceptual changes during metacontrast. Vision Research, 18(10), 13371341. https://doi.org/10.1016/0042-6989(78)90224-9

Pilling, M., \& Gellatly, A. (2009). Target visibility in the standing wave illusion: Is masktarget shape similarity important? Perception, 38(1), 5-16. https://doi.org/10.1068/p5590

Pins, D., \& ffytche, D. (2003). The neural correlates of conscious vision. Cerebral Cortex, 13(5), 461-474. https://doi.org/10.1093/cercor/13.5.461

Pitts, M. A., \& Martínez, A. (2014). Contour integration: Sensory, perceptual, and attentionbased ERP components. In Cognitive Electrophysiology of Attention: Signals of the Mind (pp. 178-189). San Diego: Academic Press. https://doi.org/10.1016/B978-0-12-398451$7.00014-2$

Pitts, M. A., Metzler, S., \& Hillyard, S. A. (2014). Isolating neural correlates of conscious perception from neural correlates of reporting one's perception. Frontiers in Psychology, 5, 1078. https://doi.org/10.3389/fpsyg.2014.01078

Purcell, D. G., \& Dember, W. N. (1968). The relation of phenomenal brightness reversal and re-reversal to backward masking and recovery. Perception \& Psychophysics, 3(4), 290292. https://doi.org/10.3758/BF03212748

R Core Team. (2014). R: A Language and Environment for Statistical Computing. Vienna, Austria. Retrieved from http://www.R-project.org/

Railo, H., \& Koivisto, M. (2009). The electrophysiological correlates of stimulus visibility and metacontrast masking. Consciousness and Cognition, 18(3), 794-803. https://doi.org/10.1016/j.concog.2009.01.006

Railo, H., Koivisto, M., \& Revonsuo, A. (2011). Tracking the processes behind conscious perception: A review of event-related potential correlates of visual consciousness. Consciousness and Cognition, 20(3), 972-983. https://doi.org/10.1016/j.concog.2011.03.019 
Ramachandran, V. S., \& Cobb, S. (1995). Visual attention modulates metacontrast masking. Nature, 373, 66-68.

Ramsøy, T. Z., \& Overgaard, M. (2004). Introspection and subliminal perception. Phenomenology and the Cognitive Sciences. (3), 1-23.

Reeves, A. (1982). Metacontrast u-shaped functions derive from two monotonic processes. Perception, 11, 415-426.

Reingold, E. M., \& Merikle, P. M. (1988). Using direct and indirect measures to study perception without awareness. Perception \& Psychophysics, 44(6), 563-575. https://doi.org/10.3758/BF03207490

Ro, T., Breitmeyer, B. G., Burton, P., Singhal, N. S., \& Lane, D. (2003). Feedback contributions to visual awareness in human occipital cortex. Current Biology, 13(12), 1038-1041. https://doi.org/10.1016/S0960-9822(03)00337-3

Ruchkin, D. S., Canoune, H. L., Johnson, R., \& Ritter, W. (1995). Working memory and preparation elicit different patterns of slow wave event-related brain potentials. Psychophysiology, 32, 399-410.

Rüter, J., Kammer, T., \& Herzog, M. H. (2010). When transcranial magnetic stimulation (TMS) modulates feature integration. The European Journal of Neuroscience, 32(11), 1951-1958. https://doi.org/10.1111/j.1460-9568.2010.07456.x

Rutiku, R., Martin, M., Bachmann, T., \& Aru, J. (2015). Does the P300 reflect conscious perception or its consequences? Neuroscience, 298, 180-189. https://doi.org/10.1016/j.neuroscience.2015.04.029

Sackur, J. (2011). Dynamics of visual masking revealed by second-order metacontrast. Journal of Vision, 11(4), 10. https://doi.org/10.1167/11.4.10

Sackur, J. (2013). Two dimensions of visibility revealed by multidimensional scaling of metacontrast. Cognition, 126(2), 173-180. https://doi.org/10.1016/j.cognition.2012.09.013

Salti, M., Bar-Haim, Y., \& Lamy, D. (2012). The P3 component of the ERP reflects conscious perception, not confidence. Consciousness and Cognition, 21(2), 961-968. https://doi.org/10.1016/j.concog.2012.01.012

Samaha, J., \& Postle, B. R. (2015). The speed of alpha-band oscillations predicts the temporal resolution of visual perception. Current Biology, 25(22), 2985-2990. https://doi.org/10.1016/j.cub.2015.10.007 
Sandberg, K., Bibby, B. M., \& Overgaard, M. (2013). Measuring and testing awareness of emotional face expressions. Consciousness and Cognition, 22(3), 806-809. https://doi.org/10.1016/j.concog.2013.04.015

Sandberg, K., Bibby, B. M., Timmermans, B., Cleeremans, A., \& Overgaard, M. (2011). Measuring consciousness: Task accuracy and awareness as sigmoid functions of stimulus duration. Consciousness and Cognition, 20(4), 1659-1675. https://doi.org/10.1016/j.concog.2011.09.002

Sandberg, K., Timmermans, B., Overgaard, M., \& Cleeremans, A. (2010). Measuring consciousness: Is one measure better than the other? Consciousness and Cognition, 19(4), 1069-1078. https://doi.org/10.1016/j.concog.2009.12.013

Scharnowski, F., Hermens, F., Kammer, T., Oğmen, H., \& Herzog, M. H. (2007). Feature fusion reveals slow and fast visual memories. Journal of Cognitive Neuroscience, 19(4), 632-641. https://doi.org/10.1162/jocn.2007.19.4.632

Scheerer, E. (1973). Integration, interruption and processing rate in visual backward masking. Psychologische Forschung, 36(1), 71-93. https://doi.org/10.1007/BF00424655

Scheerer, E., \& Bongartz, W. (1973). Integration, interruption and processing rate in visual backward masking. Psychologische Forschung, 36, 95-115.

Schiller, P. H., \& Smith, M. C. (1966). Detection in metacontrast. Journal of Experimental Psychology, 71(1), 32-39. https://doi.org/10.1037/h0022617

Schmidt, T., \& Vorberg, D. (2006). Criteria for unconscious cognition: Three types of dissociation. Perception \& Psychophysics, 68(3), 489-504.

Schwiedrzik, C. M., Singer, W., \& Melloni, L. (2009). Sensitivity and perceptual awareness increase with practice in metacontrast masking. Journal of Vision, 9(10), 18. https://doi.org/10.1167/9.10.18

Schwiedrzik, C. M., Singer, W., \& Melloni, L. (2011). Subjective and objective learning effects dissociate in space and in time. Proceedings of the National Academy of Sciences, 108(11), 4506-4511. https://doi.org/10.1073/pnas.1009147108

Searle, J. R. (2000). Consciousness. Annual Reviews Neuroscience, 23, 557-578.

Sergent, C., Baillet, S., \& Dehaene, S. (2005). Timing of the brain events underlying access to consciousness during the attentional blink. Nature Neuroscience, 8(10), 1391-1400. https://doi.org/10.1038/nn1549 
Sergent, C., \& Dehaene, S. (2004). Is consciousness a gradual phenomenon? Evidence for an all-or-none bifurcation during the attentional blink. Psychological Science, 15(11), 720728. https://doi.org/10.1111/j.0956-7976.2004.00748.x

Sherrick, M. F., \& Dember, W. N. (1970). Completeness and spatial distribution of mask contours as factors in visual backward masking. Journal of Experimental Psychology, 84(1), 179-180. https://doi.org/10.1037/h0028928

Simons, D. J. (2000). Current approaches to change blindness. Visual Cognition, 7(1-3), 1-15. https://doi.org/10.1080/135062800394658

Spencer, T. J., \& Shuntich, R. (1970). Evidence for an interruption theory of backward masking. Journal of Experimental Psychology: General, 85(2), 198-203.

Spillmann, L. (2009). Phenomenology and neurophysiological correlations: Two approaches to perception research. Vision Research, 49(12), 1507-1521. https://doi.org/10.1016/j.visres.2009.02.022

Sterzer, P., Haynes, J.-D., \& Rees, G. (2006). Primary visual cortex activation on the path of apparent motion is mediated by feedback from hMT+/V5. NeuroImage, 32(3), 1308-1316. https://doi.org/10.1016/j.neuroimage.2006.05.029

Stewart, A. L., Purcell, D. G., \& Pinkham, R. S. (2011). Nonmonotone backward masking functions and brightness reversals. Attention, Perception, \& Psychophysics, 73(7), 2180 2196. https://doi.org/10.3758/s13414-011-0166-y

Stigler, R. (1910). Chronophotische Studien über den Umgebungskontrast. Pflüger's Archiv Für Die Gesamte Physiologie Des Menschen Und Der Tiere, 134(6), 365-435. https://doi.org/10.1007/BF01680352

Stober, S. R., Brussell, E. M., \& Komoda, M. K. (1978). Differential effects of metacontrast on target brightness and clarity. Bulletin of the Psychonomic Society, 12(6), 433-436. https://doi.org/10.3758/BF03329729

Stoper, A. E., \& Banffy, S. (1977). Relation of split apparent motion to metacontrast. Journal of Experimental Psychology: Human Perception and Performance, 3(2), 258-277. https://doi.org/10.1037//0096-1523.3.2.258

Stoper, A. E., \& Mansfield, J. G. (1978). Metacontrast and paracontrast suppression of a contourless area. Vision Research, 18(12), 1669-1674. https://doi.org/10.1016/00426989(78)90259-6 
Szczepanowski, R., \& Pessoa, L. (2007). Fear perception: Can objective and subjective awareness measures be dissociated? Journal of Vision, 7(4), 10. https://doi.org/10.1167/7.4.10

Szczepanowski, R., Traczyk, J., Wierzchoń, M., \& Cleeremans, A. (2013). The perception of visual emotion: Comparing different measures of awareness. Consciousness and Cognition, 22(1), 212-220. https://doi.org/10.1016/j.concog.2012.12.003

Tapia, E., \& Beck, D. M. (2014). Probing feedforward and feedback contributions to awareness with visual masking and transcranial magnetic stimulation. Frontiers in Psychology, 5, 1173. https://doi.org/10.3389/fpsyg.2014.01173

Tapia, E., \& Breitmeyer, B. G. (2011). Visual consciousness revisited: Magnocellular and parvocellular contributions to conscious and nonconscious vision. Psychological Science, 22(7), 934-942. https://doi.org/10.1177/0956797611413471

Tapia, E., Breitmeyer, B. G., \& Jacob, J. (2011). Metacontrast masking with texture-defined second-order stimuli. Vision Research, 51(23-24), 2453-2461. https://doi.org/10.1016/j.visres.2011.10.010

Timmermans, B., \& Cleeremans, A. (2015). How can we measure awareness? An overview of current methods. In M. Overgaard (Ed.), Behavioral Methods in Consciousness Research (pp. 21-46). Oxford University Press.

Toch, H. H. (1956). The perceptual elaboration of stroboscopic presentations. The American Journal of Psychology, 69(3), 345-358.

Treisman, A. (1996). The binding problem. Current Opinion in Neurobiology, 6(2), 171-178. https://doi.org/10.1016/S0959-4388(96)80070-5

Trevethan, C. T., Sahraie, A., \& Weiskrantz, L. (2007). Form discrimination in a case of blindsight. Neuropsychologia, 45(9), 2092-2103. https://doi.org/10.1016/j.neuropsychologia.2007.01.022

Tsuchiya, N., Wilke, M., Frässle, S., \& Lamme, V. A. F. (2015). No-report paradigms: Extracting the true neural correlates of consciousness. Trends in Cognitive Sciences, 19(12), 757-770. https://doi.org/10.1016/j.tics.2015.10.002

Turvey, M. T. (1973). On peripheral and central processes in vision: Inferences from an information-processing analysis of masking with patterned stimuli. Psychological Review, 80(1), 1-52. https://doi.org/10.1037/h0033872 
Uttal, W. R. (1970). On the physiological basis of masking with dotted visual noise. Perception \& Psychophysics, 7(6), 321-327. https://doi.org/10.3758/BF03208656

Van Aalderen-Smeets, S. I., Oostenveld, R., \& Schwarzbach, J. (2006). Investigating neurophysiological correlates of metacontrast masking with magnetoencephalography. Advances in Cognitive Psychology, 2(1), 21-35. https://doi.org/10.2478/v10053-008-0042$\mathrm{Z}$

Varela, F. J. (1996). Neurophenomenology: A methodological remedy for the hard problem. Journal of Consciousness Studies, 3(4), 330-349.

Varela, F. J., \& Shear, J. (1999). First-person methodologies: What, why, how? Journal of Consciousness Studies, 6(2-3), 1-14.

Velmans, M. (2007). Heterophenomenology versus critical phenomenology. Phenomenology and the Cognitive Sciences, 6(1-2), 221-230. https://doi.org/10.1007/s11097-006-9033-z

Ventura, J. (1980). Foveal metacontrast: I. Criterion content and practice effects. Journal of Experimental Psychology: Human Perception and Performance, 6(3), 473-485.

Vermersch, P. (1999). Introspection as practice. Journal of Consciousness Studies, 6(2-3), 1742.

Vernoy, M. W. (1976). Masking by pattern in random-dot stereograms. Vision Research, 16, $1183-1184$.

Vogel, E. K., \& Machizawa, M. G. (2004). Neural activity predicts individual differences in visual working memory capacity. Nature, 428(6984), 748-751. https://doi.org/10.1038/nature02447

Vorberg, D., Mattler, U., Heinecke, A., Schmidt, T., \& Schwarzbach, J. (2004). Invariant time course of priming with and without awareness. In C. Kaernbach, E. Schröger, \& H. Müller (Eds.), Psychophysics beyond sensation: Laws and invariants of human cognition (pp. 273-290). Hillsdale, NJ: Erlbaum.

Watson, J. B. (1931). Psychology as the behaviorist views it. Psychological Review, 2(20), $158-177$.

Weiskrantz, L., Barbur, J. L., \& Sahraie, A. (1995). Parameters affecting conscious versus unconscious visual discrimination with damage to the visual cortex (V1). Proceedings of the National Academy of Sciences, 92(13), 6122-6126. https://doi.org/10.1073/pnas.92.13.6122 
Weisstein, N. (1968). A Rashevsky-Landahl neural net: Simulation of metacontrast. Psychological Review, 75(6), 494-521. https://doi.org/10.1037/h0026769

Weisstein, N. (1972). Metacontrast. In D. Jameson \& L. Hurvich (Eds.), Handbook of sensory physiology. Berlin: Springer-Verlag.

Weisstein, N., \& Growney, R. L. (1969). Apparent movement and metacontrast: A note on Kahneman's formulation. Perception \& Psychophysics, 5(6), 321-328.

Weisstein, N., Ozog, G., \& Szoc, R. (1975). A comparison and elaboration of two models of metacontrast. Psychological Review, 82(5), 325-343. https://doi.org/10.1037/0033295X.82.5.325

Werner, H. (1935). Studies on contour: I. Qualitative analyses. The American Journal of Psychology, 49(1), 40-46.

Wertheimer, M. (1912). Experimentelle Studien über das Sehen von Bewegung. Zeitschrift Für Psychologie Und Physiologie Der Sinnesorgane, 61(1), 161-265.

Wiens, S. (2008). Concepts of visual consciousness and their measurement. Advances in Cognitive Psychology, 3(1-2), 349-359. https://doi.org/10.2478/v10053-008-0035-y

Wilenius, M. E., \& Revonsuo, A. T. (2007). Timing of the earliest ERP correlate of visual awareness. Psychophysiology, 44(5), 703-710. https://doi.org/10.1111/j.14698986.2007.00546.x

Witt, H. (2010). Introspektion. In G. Mey \& K. Mruck (Eds.), Handbuch Qualitative Forschung in der Psychologie (pp. 491-505). Wiesbaden: VS Verlag für Sozialwissenschaften. https://doi.org/10.1007/978-3-531-92052-8_35

Woodman, G. F., \& Luck, S. J. (2003). Dissociation among attention, perception, and awareness during object-substitution masking. Psychological Science, 14(6), 605-611. https://doi.org/10.1023/B:TRAG.0000005082.29212.cf

Zahavi, D. (2003). Husserl's phenomenology. Cultural memory in the present. Stanford: Stanford University Press.

Zehetleitner, M., \& Rausch, M. (2013). Being confident without seeing: What subjective measures of visual consciousness are about. Attention, Perception, \& Psychophysics, 75(7), 1406-1426. https://doi.org/10.3758/s13414-013-0505-2

Zeki, S. (2015). A massively asynchronous, parallel brain. Philosophical Transactions of the Royal Society B: Biological Sciences, 370(1668). https://doi.org/10.1098/rstb.2014.0174 


\section{Appendix I - Experiment 1}

\section{Table A1}

Exemplary idiosyncratic descriptions of different participants for each perceptual category (translated in English).

\section{Target inside Mask}

The inner shape of the mask is clearly filled with the target. The target disappears, but is clearly visible beforehand.

Target fills the inside of the mask briefly, shape of target stimulus cannot be determined.

Target black square which seems to luminesce, appears again clearly inside the white part of the mask, white square inside the white part seems to pulsate once.

\section{Target before Mask}

Target difficult to recognize and perceived as separated from mask

Target clearly recognizable in its shape, corners and edges clearly visible. First target faded in, then mask, two well separable impressions.

Target clearly recognizable. The target and mask appears separately, i.e. target before mask. But because target stimulus is so concise, it is also visible inside mask. Target black, all corners and edges to recognize.

\section{Dark Target}

Shape of the target was not accurately detected, only a non-geometric, dark spot. Shape of the spot is different. Partly only short flashing seen.

Filled, diamond-shaped target, which look exactly like the sample pictures of the stimuli.

Mask and target well to recognize, both seen at the same time, nevertheless I could separate both well

\section{Bright Target}

Black mask with white target stimulus, both diamonds, focus on mask

A white diamond is visible in the white star.

White square in white star. Square appears larger than remaining part of the white star.

\section{No Target}

Target is not seen at all.

Impression as if the mask is presented twice. Target is not perceived.

No target perceived. If you didn't know that the target stimulus was shown in advance, then you would have only reported mask presentation without having recognized anything strange. 
Table A1 (continued)

\section{Rotation}

Feeling that two pictures follow each other and the frame of the mask rotates/a movement is created. This only refers to the frame, the target stimulus is neither perceived as a form nor as a black spot.

Rotation in the transition from target to mask.

Two different forms create a motion in the sequence. It seems as if the picture rotates.

\section{Expansion}

The target stimulus is first small in the inner star of the mask and then increases to the boundary of the mask. First the target stimulus is visible, then the mask, then both together.

At the fixation point, target stimulus has grown, increasing in size in all directions. Enlargement, occurred when the shape of the target stimulus and the mask coincided or when a non-geometric spot (dark) is perceived; increases to the size of the frame of the mask. 


\section{Table A2}

$P$-values for each effect of the Randomization test, calculated separately for Rater 1 and Rater 2.

\begin{tabular}{|c|c|c|}
\hline Target inside Mask & Rater 1 & Rater 2 \\
\hline Congruent vs incongruent & .03 & .46 \\
\hline SOA linear & .21 & .09 \\
\hline SOA quadratic & .35 & .21 \\
\hline Congruent vs incongruent $*$ SOA linear & .58 & .37 \\
\hline Congruent vs incongruent $*$ SOA quadratic & .22 & .02 \\
\hline Target before Mask & Rater 1 & Rater 2 \\
\hline Congruent vs incongruent & .006 & .50 \\
\hline SOA linear & .33 & .15 \\
\hline SOA quadratic & .16 & .025 \\
\hline Congruent vs incongruent $*$ SOA linear & .002 & .079 \\
\hline Congruent vs incongruent $*$ SOA quadratic & .006 & .019 \\
\hline Dark Target & Rater 1 & Rater 2 \\
\hline Congruent vs incongruent & .13 & .11 \\
\hline SOA linear & .54 & .83 \\
\hline SOA quadratic & .13 & .03 \\
\hline Congruent vs incongruent $*$ SOA linear & .08 & .25 \\
\hline Congruent vs incongruent $*$ SOA quadratic & .82 & .90 \\
\hline Bright Target & Rater 1 & Rater 2 \\
\hline Congruent vs incongruent & .24 & .44 \\
\hline SOA linear & 1 & .49 \\
\hline SOA quadratic & .80 & .71 \\
\hline Congruent vs incongruent $*$ SOA linear & .007 & .55 \\
\hline Congruent vs incongruent $*$ SOA quadratic & .098 & .57 \\
\hline
\end{tabular}


Table A2 (continued)

\begin{tabular}{lcc}
\hline \multicolumn{1}{c}{ No Target } & Rater 1 & Rater 2 \\
\hline Congruent vs incongruent & .98 & .78 \\
SOA linear & .71 & .38 \\
SOA quadratic & .001 & .001 \\
Congruent vs incongruent * SOA linear & .30 & .26 \\
Congruent vs incongruent * SOA quadratic & .58 & .79 \\
\hline \multicolumn{1}{c}{ Rotation } & Rater 1 & Rater 2 \\
\hline Congruent vs incongruent & .001 & .0012 \\
SOA linear & .001 & .0016 \\
SOA quadratic & .007 & .012 \\
Congruent vs incongruent * SOA linear & .001 & .0004 \\
Congruent vs incongruent * SOA quadratic & .008 & .008 \\
\hline Expansion & Rater 1 & Rater 2 \\
\hline Congruent vs incongruent * SOA quadratic & .45 & .66 \\
\hline SOA linear & .04 & .08 \\
SOA quadratic & .04 & .03 \\
\hline
\end{tabular}


A

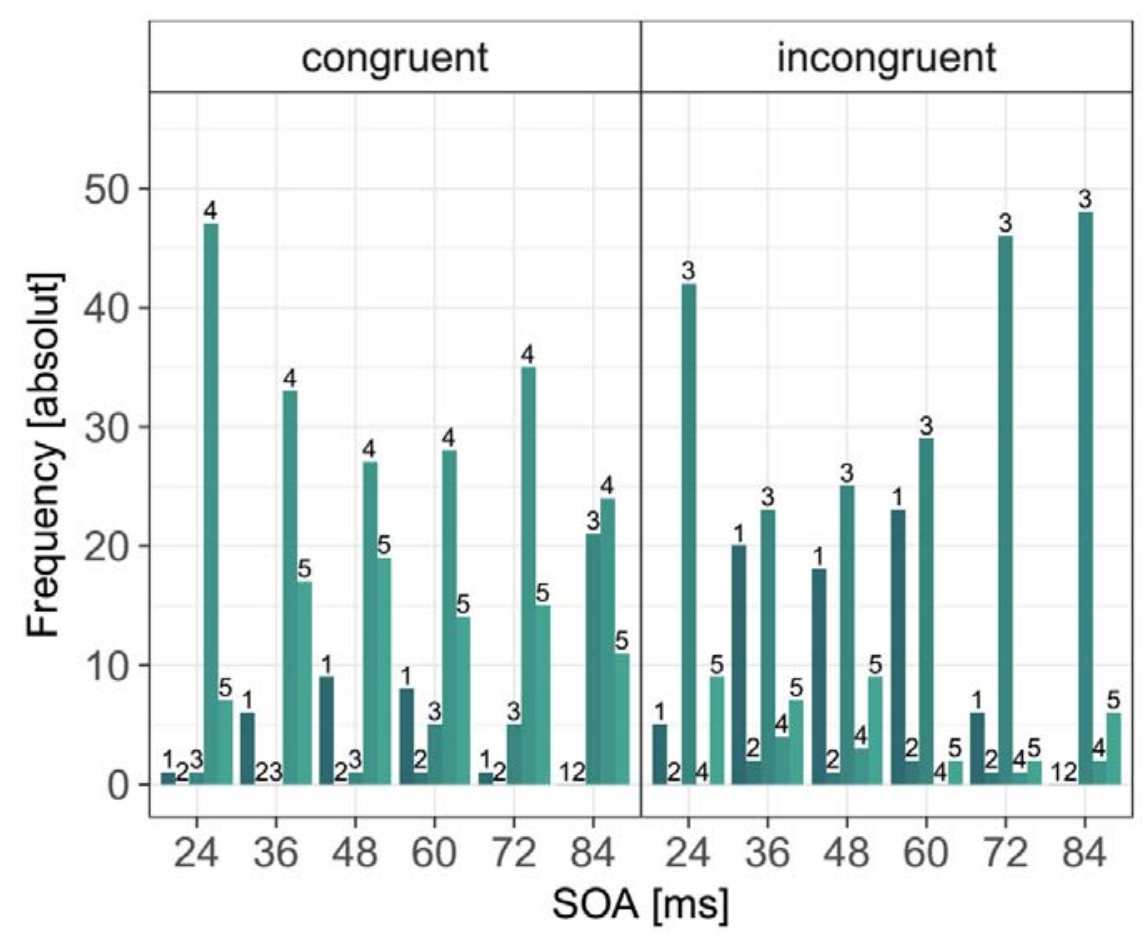

B

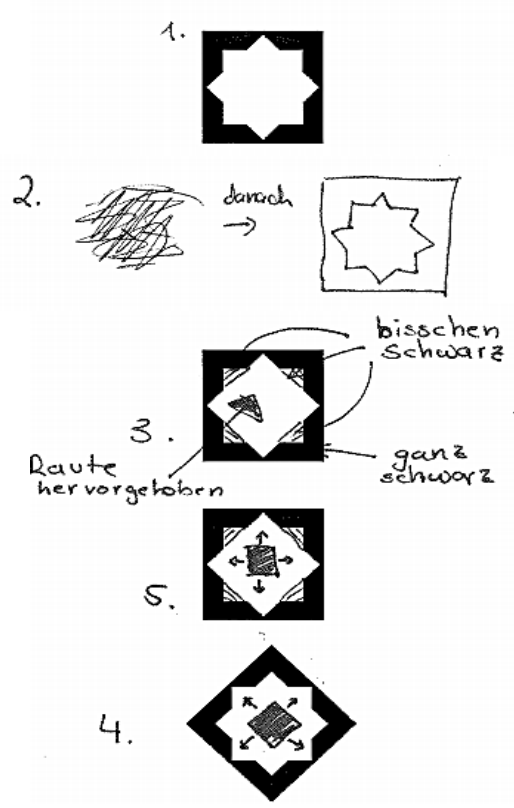

C (1) Mask: No perception of the target, only the mask was visible.

(2) Spot: Target is difficult to perceive. Target is segregated from mask.

(3) Star: Some parts of the star were highlighted.

(4) Continuum: Continuous transition between target and mask.

(5) Combination: Mix, some parts of the star were highlighted (" 3 ”) and a continuous transition between target and mask ("4").

Figure A1. A Exemplary time courses of idiosyncratic descriptions of one participant at Experiment 1a. B The drawings for each idiosyncratic description and $\mathbf{C}$ the arbitrary label as well as the descriptions. 
A

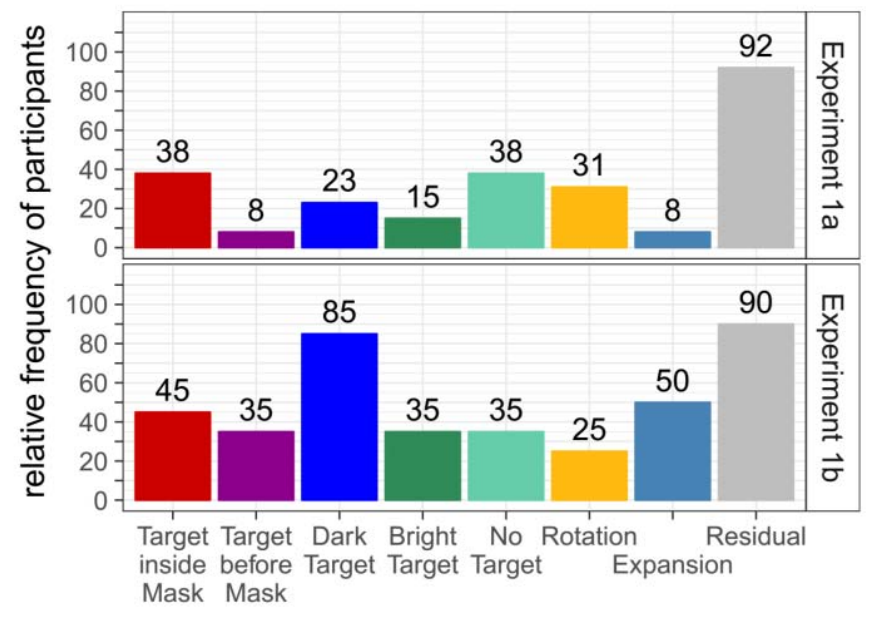

B

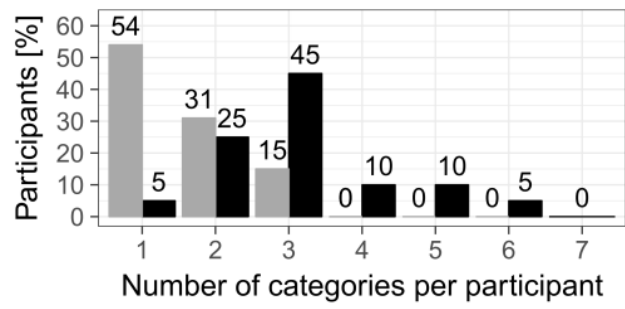

Figure A2. A Percentage of participants, whose description were rated inside the particular category of Experiment 1a (top) and Experiment 1b (bottom). B Percentage of participants reporting different number of categories exclusive the residual category for Experiment 1a (gray bars) and Experiment $1 \mathrm{~b}$ (black bars). The absolute value of participants in Experiment 1a was $N=13$ and $N=20$ in Experiment 1b. Results based on Rating 2. 


\section{Appendix II - Experiment 2}

\section{Supplementary information}

\section{Detailed instruction of Session 1 of Experiment 2}

The first session served as training, to provide an understanding for the perception of the categories. Participants were informed about the shape of the stimuli, including the information that the size of the target fits neatly in the star-shaped inner contour of the mask. It was also mentioned, that there were any not nearer specified differences between trials, which causes differences in the perception. Differences in the stimulations should make differences in the perception more plausible for participants, and therefore increase the motivation to perform the difficult perceptual task. To avoid exclusion strategies for the different categories, it was not mentioned, that only the query varies across blocks, whereas the experimental manipulations were equal across blocks. In addition, the following instructions were given, at one hand to avoid a demotivation because of the difficulty and on the other hand to ensure that participants do not feel forced to affirm the categories, if they do not perceive them: "Because the target is hardly visible, it is difficult to perceive the different categories and there are individual differences in the perception. Therefore, some categories may be easier for you to perceive than others and you may perceive some categories faster than the others. Anyway, do not stop searching for the categories, some of them you may perceive only at the end of the first session or even after a few sessions. At least, it is also possible that you may never perceive some of the categories."

After performing a certain number of trials participants had to describe their individual perception of the particular category. Because of the difficulty of the task, participants were often corrected or the definitions were repeated verbally by the instructor, who stayed in the room for the first session. To avoid a frustration of the participants by the frequent corrections, the following information was also given at the beginning of the first session: "The aim of the first session is to provide an understanding of the perceptions of the different categories. Therefore, I'll be asking you to describe your individual experiences of the particular category. It is important to use your own words and not just stick to the definition of the particular category. I'll interview you to ensure that there is no misunderstanding about what is meant by the different categories. If though, I'll correct you. According to the experience this is a very difficult task, and normally subjects will be often corrected. Do not get discourage from the corrections." 


\section{Definition of categories}

Categories were defined as follows. Target inside Mask: Target and mask are perceived simultaneously, at the inner white star of the mask, the target is visible. The target can be perceived as a bright or dark stimulus, but it is not important to perceive the form of the target. Target before Mask: First the target is presented, which is followed by the mask, both stimuli are perceived temporal successively. The target can be perceived as a bright or dark stimulus, but it is not important to perceive the form of the target. Dark Target: A black or gray target is perceived, which is either presented simultaneously or successive with the mask. It is not important to perceive the form of the target. Bright Target: A target brighter than the background is perceived, which is either presented simultaneously or successive with the mask. It is not important to perceive the form of the target. No Target: No target was visible, only the Mask was visible. Rotation: Either only the mask is visible, which is rotation or in the transition between target and mask a rotation is visible. Expansion: The target is first perceived smaller and then grows to its original size. The target is growing to the mask. The target is either presented simultaneously or successive with the mask. 


\section{Table B1}

Summary of the linear mixed effect model calculated for discrimination performance $(N=24$, Number of observations: 144).

\begin{tabular}{lcccc}
\hline B & SE & $\boldsymbol{t}$-value & $\boldsymbol{p}$-value \\
\hline Random effect of subject: variance $=0.24(\mathrm{SD}=0.49)$ & \\
Random effect of SOA linear: variance $=0.21(\mathrm{SD}=0.45)$ & \\
Random effect of SOA quadratic: variance $=0.02(\mathrm{SD}=0.15)$ \\
SOA linear \\
-0.45 & 0.10 & -4.63 & $<.001$ \\
SOA quadratic & 0.33 & 0.04 & 8.07 & $<.001$ \\
\hline
\end{tabular}




\section{Table B2}

Summary of linear mixed effect models calculated for the relationship between discrimination performance and each category separately $(N=24$, Number of observations: 144).

\begin{tabular}{|c|c|c|c|c|}
\hline Target inside Mask & B & SE & $t$-value & $p$-value \\
\hline \multicolumn{5}{|c|}{$\begin{array}{l}\text { Random effect of subject: variance }=0.2(\mathrm{SD}=0.45) \\
\text { Random effect of SOA linear: variance }=0.20(\mathrm{SD}=0.44) \\
\text { Random effect of SOA quadratic: variance }=0.02(\mathrm{SD}=0.13)\end{array}$} \\
\hline SOA linear & -0.30 & 0.1 & -3.04 & .005 \\
\hline SOA quadratic & 0.19 & 0.04 & 4.33 & $<.001$ \\
\hline Category & .20 & 0.07 & 2.69 & .009 \\
\hline SOA linear $*$ Category & -0.19 & 0.06 & -3.37 & .001 \\
\hline SOA quadratic $*$ Category & 0.08 & 0.03 & 2.32 & .02 \\
\hline Target before Mask & B & SE & $t$-value & $p$-value \\
\hline \multicolumn{5}{|c|}{$\begin{array}{l}\text { Random effect of subject: variance }=0.28(\mathrm{SD}=0.53) \\
\text { Random effect of SOA linear: variance }=0.16(\mathrm{SD}=0.40) \\
\text { Random effect of SOA quadratic: variance }=0.02(\mathrm{SD}=0.15)\end{array}$} \\
\hline SOA linear & -0.53 & 0.09 & -6.2 & $<.001$ \\
\hline SOA quadratic & 0.25 & 0.05 & 5.43 & $<.001$ \\
\hline Category & 0.33 & 0.08 & 4.22 & $<.001$ \\
\hline SOA linear $*$ Category & -0.01 & 0.05 & -0.34 & .74 \\
\hline SOA quadratic $*$ Category & 0.01 & 0.03 & 0.31 & .76 \\
\hline Dark Target & B & SE & $t$-value & $p$-value \\
\hline \multicolumn{5}{|c|}{$\begin{array}{l}\text { Random effect of subject: variance }=0.31(\mathrm{SD}=0.56) \\
\text { Random effect of SOA linear: variance }=0.12(\mathrm{SD}=0.34) \\
\text { Random effect of SOA quadratic: variance }=0.09(\mathrm{SD}=0.13)\end{array}$} \\
\hline SOA linear & -0.44 & 0.07 & -5.88 & $<.001$ \\
\hline SOA quadratic & 0.17 & 0.04 & 3.91 & $<.001$ \\
\hline Category & 0.44 & 0.07 & 6.44 & $<.001$ \\
\hline SOA linear * Category & -0.06 & 0.04 & -1.31 & .19 \\
\hline SOA quadratic * Category & 0.04 & 0.03 & 1.33 & .19 \\
\hline Bright Target & B & $\mathbf{S E}$ & $t$-value & $p$-value \\
\hline \multicolumn{5}{|c|}{$\begin{array}{l}\text { Random effect of subject: variance }=0.26(\mathrm{SD}=0.51) \\
\text { Random effect of SOA linear: variance }=0.21(\mathrm{SD}=0.46) \\
\text { Random effect of SOA quadratic: variance }=0.01(\mathrm{SD}=0.12)\end{array}$} \\
\hline SOA linear & -0.45 & 0.10 & -4.57 & $<.001$ \\
\hline SOA quadratic & 0.33 & 0.04 & 8.92 & $<.001$ \\
\hline Category & -0.04 & 0.09 & -0.47 & .64 \\
\hline SOA linear $*$ Category & -0.01 & 0.07 & -0.14 & .89 \\
\hline SOA quadratic * Category & 0.07 & 0.03 & 2.04 & .048 \\
\hline
\end{tabular}


Table B2 (continued)

\begin{tabular}{|c|c|c|c|c|}
\hline No Target & B & SE & $t$-value & $p$-value \\
\hline \multicolumn{5}{|c|}{$\begin{array}{l}\text { Random effect of subject: variance }=0.21(\mathrm{SD}=0.46) \\
\text { Random effect of SOA linear: variance }=0.13(\mathrm{SD}=0.35) \\
\text { Random effect of SOA quadratic: variance }=0.02(\mathrm{SD}=0.15)\end{array}$} \\
\hline SOA linear & -0.46 & 0.08 & -5.94 & $<.001$ \\
\hline SOA quadratic & 0.19 & 0.05 & 4.15 & $<.001$ \\
\hline Category & -0.36 & 0.06 & -5.65 & $<.001$ \\
\hline SOA linear * Category & 0.06 & 0.04 & 1.63 & .11 \\
\hline SOA quadratic * Category & -0.03 & 0.03 & -0.93 & .36 \\
\hline Rotation & B & SE & $t$-value & $p$-value \\
\hline \multicolumn{5}{|c|}{$\begin{array}{l}\text { Random effect of subject: variance }=0.24(\mathrm{SD}=0.49) \\
\text { Random effect of SOA linear: variance }=0.20(\mathrm{SD}=0.45) \\
\text { Random effect of SOA quadratic: variance }=0.03(\mathrm{SD}=0.18)\end{array}$} \\
\hline SOA linear & -0.37 & 0.11 & -3.53 & .001 \\
\hline SOA quadratic & 0.25 & 0.05 & 4.58 & $<.001$ \\
\hline Category & 0.002 & 0.08 & 0.02 & .98 \\
\hline SOA linear $*$ Category & 0.18 & 0.06 & 2.79 & .006 \\
\hline SOA quadratic * Category & -0.13 & 0.04 & -3.39 & .001 \\
\hline Expansion & B & SE & $t$-value & $p$-value \\
\hline \multicolumn{5}{|c|}{$\begin{array}{l}\text { Random effect of subject: variance }=0.24(\mathrm{SD}=0.49) \\
\text { Random effect of SOA linear: variance }=0.20(\mathrm{SD}=0.45) \\
\text { Random effect of SOA quadratic: variance }=0.02(\mathrm{SD}=0.16)\end{array}$} \\
\hline SOA linear & -0.39 & 0.10 & -4.01 & $<.001$ \\
\hline SOA quadratic & 0.28 & 0.05 & 5.91 & $<.001$ \\
\hline Category & 0.07 & 0.08 & 0.86 & .40 \\
\hline SOA linear $*$ Category & -0.10 & 0.06 & -1.77 & .08 \\
\hline SOA quadratic * Category & 0.07 & 0.04 & 1.96 & .055 \\
\hline
\end{tabular}




\section{Appendix III - Experiment 3}

\section{Supplementary information}

\section{Detailed instructions of Experiment 3}

The perceptual categories were described as follows: An Expansion as the perception that the target first appears small and then grows to its actual size, a Target inside Mask as the perception of simultaneity of target and mask, which leads to the impression of a target integrated inside the white star of the mask. A Rotation was defined as the perception of a rotational movement, which was limited to the mask alone or to an interplay between target and mask. A Target before Mask was described as the perception of two temporally segregated objects, first the target and second the mask.

If participants were unable to perceive a category or a combination of two categories during the experiment, they were informed about subjective variability in the ability to perceive the different categories. This was done to prevent the participants from affirming the categories, without perceiving them. But to keep them motivate, participants were also informed that it may need one or even more sessions to perceive each category equally good. Further, they were informed that each of the four categories can be perceived in each block and that they had to distinguish properly, whether the perceived a target inside or before the mask. Nevertheless, they were requested to focus on the specified categories of the particular block. This was done because in a previous experiment (Experiment 2) participants had problems to distinguish between an integrated and a segregated target. A reason could be that they were not prompt to focus on distinguishing between those two categories.

\section{Detailed procedure of Experiment 3}

Participants reported whether they perceived a Target inside Mask and Expansion by pressing the buttons "Q", "T" as yes- and no-answer for one category and the buttons "<", "V" for the other category. For Target before Mask and Rotation the buttons "U", "Ü", were assigned as yes-and no-buttons to one category and "N", "-" to the other. The assignment of the categories to the upper and lower button row of the keyboard as well as the mapping of left and right buttons to the yes- and no-answer was counterbalanced across subjects. Participants used the index fingers and the thumbs to press the buttons of the upper and lower row, respectively. After the first answer was given, the fixation cross disappeared shortly and 
the buttons, which were assigned to the first answered category were deactivated until the second response was given. This was done to prevent participant to respond accidentally twice to the same category. To make sure that participants did not respond to the wrong categories, first, participants started each block by pressing either the particular yes-button for Expansion or the particular yes-button for Rotation. Second, after each block they were asked to answer which pair of categories they responded to in the last block by pressing the particular button. 


\section{Appendix IV - Experiment 4}
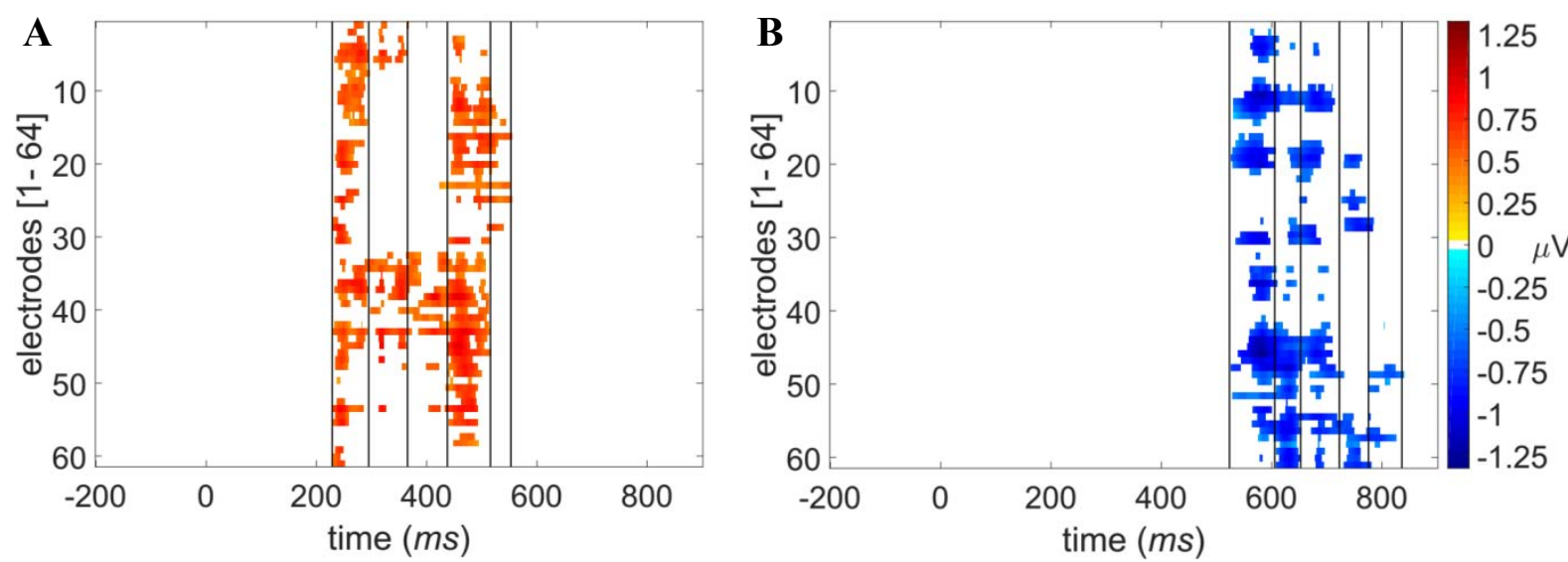

Figure D1. Electrode-time matrices visualizing the strength of activity differences, based on targetlocked grand averaged ERPs, of the cells which form the particular cluster. Cells which were not part of the cluster were masked for a better illustration of the results. The vertical bars indicate the subdivision of the particular cluster into different time ranges done by visual inspection. A Cluster 1 shows the difference in activity between trials on which "Rotation yes - Target no" was reported compared to trials on which "Rotation no - Target no" was reported. B Cluster 2 shows the difference in activity between trials on which "Rotation no - Target yes" was reported compared to trials on which "Rotation no - Target no" was reported. 


\section{List of Figures}

\section{Chapter 2 - General Methods}

Figure 2.1. A Square- and diamond-shaped target and mask stimuli. B Trial sequence. 27

\section{Chapter 3 - Visual perception is rich. Phenomenological evidence from metacontrast masking}

Figure 3.1. A Percentages of participants, whose description were rated inside the particular category. B Percentage of participants reporting different number of categories

Figure 3.2. The mean relative frequencies of reports for each perceptual category as function of SOA and Congruency for Experiment 1.

Figure 3.3. The relative frequency of reports for each participant and category as raw data. The distribution of relative frequencies for each category is expressed by the density bean.. 47

Figure 3.4. The mean relative frequencies of reports for each perceptual category as function of SOA and Congruency.

Figure 3.5. Individual masking functions and averaged discrimination sensitivity for each SOA. 53

\section{Chapter 4 - Phenomenological evidence for multiple processes underlying metacontrast masking}

Figure 4.1. A Conditional relative frequencies to report an Expansion for each level of SOA, Congruency and the Category Target inside Mask. B Odds ratio of model 6 with Expansion as response variable.

Figure 4.2. A Conditional relative frequencies to report a Target inside Mask for each level of SOA, Congruency and the Category Expansion. B Odds ratio of model 6 with Target inside Mask as response variable.

Figure 4.3. A Conditional relative frequencies to report a Rotation for each level of SOA, Congruency and the Category Target before Mask. B Odds ratio of model 6 with Rotation as response variable

Figure 4.4. A Conditional relative frequencies to report a Target before Mask for each level of SOA, Congruency and the Category Rotation. B Odds ratio of model 6 with Rotation as response variable 


\section{Chapter 5 - Neurophysiological evidence for the multidimensionality of metacontrast masking}

Figure 5.1. Mean relative frequency of Rotation (red) and Target before Mask (back) for participants which were included in the analysis after the EEG-Sessions 104

Figure 5.2. A Target-locked, grand averaged ERPs of Cluster 1. B-D Topography maps of Cluster 1 106

Figure 5.3 A Target-locked, grand averaged ERPs of Cluster 2. B-D Topography maps of Cluster 2 . 107

\section{Appendix I - Experiment 1}

Figure A1. A Exemplary time courses of idiosyncratic descriptions of one participant. B The drawings for each idiosyncratic description. C The arbitrary label as well as the descriptions.

Figure A2. A Percentages of participants, whose description were rated inside the particular category. B Percentage of participants reporting different number of categories

\section{Appendix IV - Experiment 4}

Figure D1. Electrode-time matrices visualizing the strength of activity differences, based on target-locked grand averaged ERPs, of the cells which form the particular cluster. 185 


\section{List of Tables}

\section{Chapter 3 - Visual perception is rich. Phenomenological evidence from metacontrast masking}

Table 3.1 Interrater-Reliability of Experiment $1 \mathrm{a}$ and Experiment $1 \mathrm{~b}$ and pooled across both experiments for each category. 38

Table 3.2 Summary of the generalized linear mixed effect models, calculated for each category separately. 50

\section{Chapter 4 - Phenomenological evidence for multiple processes underlying metacontrast masking}

Table 4.1 Summary of the generalized linear mixed effect model, calculated for Expansion $(N=24$, Number of observations: 24192). .73

Table 4.2 Summary of the generalized linear mixed effect model, calculated for Target inside Mask $(N=24$, Number of observations: 24192).

Table 4.3 Summary of the generalized linear mixed effect model, calculated for Rotation $(N=24$, Number of observations: 24192).

Table 4.4 Summary of the generalized linear mixed effect model, calculated for Target before Mask $(N=24$, Number of observations: 24192). 82

\section{Appendix I - Experiment 1}

Table A1 Exemplary idiosyncratic descriptions of different participants for each perceptual category. 172

Table A2 P-values for each effect of the Randomization test 174

\section{Appendix II - Experiment 2}

Table B1 Summary of the linear mixed effect model calculated for discrimination performance.

Table B2 Summary of the linear mixed effect models calculated for the relationship between discrimination performance and each category separately . 


\section{List of Abbreviations}

\begin{tabular}{|c|c|}
\hline ANOVA & Analysis of variance \\
\hline $\mathrm{cm}$ & Centimeter \\
\hline EEG & Electroencephalography \\
\hline e.g. & Exempli gratia (meaning “for example") \\
\hline EOG & Electro-oculography \\
\hline ERP & Event-related potentials \\
\hline fMRI & Functional magnetic resonance imaging \\
\hline $\mathrm{Hz}$ & Hertz \\
\hline ICA & Independent component analysis \\
\hline ISI & Interstimulus interval \\
\hline IT & Inferior temporal cortex \\
\hline LGN & Lateral geniculate nucleus \\
\hline LP & Late positivity \\
\hline $\mathrm{ms}$ & Milliseconds \\
\hline $\mathrm{MT}+$ & Middle temporal visual area \\
\hline $\mathrm{NCC}$ & Neural correlates of consciousness \\
\hline OSM & Object substitution masking \\
\hline PFC & Prefrontal cortex \\
\hline rTMS & Repetitive transcranial magnetic stimulation \\
\hline SOA & Stimulus onset asynchrony \\
\hline VAN & Visual awareness negativity \\
\hline V1 & Primary visual cortex \\
\hline $\mathrm{V} 2$ & Secondary visual cortex \\
\hline V4 & Visual area V4 \\
\hline V5 & Visual area V5 \\
\hline
\end{tabular}




\section{Eigenständigkeitserklärung Declaration of Academic Honesty}

Hiermit erkläre ich, dass die vorliegende Arbeit ohne unzulässige Hilfe und ohne Benutzung anderer als der angegebenen Hilfsmittel angefertigt wurde und dass die aus fremden Quellen direkt oder indirekt übernommenen Gedanken in der Arbeit als solche kenntlich gemacht worden sind.

I hereby declare that this dissertation is my own work. Information derived from the published and unpublished work of others has been acknowledged in the text and a list of references is given in the bibliography.

Goettingen, 14.01.2019

Nora Meike Koster 